\title{
Properties of oxide thin films and their adsorption behavior studied by scanning tunneling microscopy and conductance spectroscopy
}

\author{
Niklas Nilius \\ Fritz-Haber Institut der Max-Planck Gesellschaft \\ Faradayweg 4-6, D-14195 Berlin, Germany
}

\begin{abstract}
The preparation of thin oxide films on metal supports is a versatile approach to explore the properties of oxide materials that are otherwise inaccessible to most surface science techniques due to their insulating nature. Although substantial progress has been made in the characterization of oxide surfaces with spatially averaging techniques, a local view is often essential to provide comprehensive understanding of such systems. The scanning tunneling microscope (STM) is a powerful tool to obtain atomic-scale information on the growth behavior of oxide films, the resulting surface morphology and defect structure. Furthermore, the binding configuration and spatial distribution of adsorbates on the oxide surface, as well as their electronic and optical properties can be probed with the STM and embedded spectroscopic techniques.

This article surveys state-of-the-art STM experiments aiming for an investigation of surface properties of oxide materials as well as their interaction with individual adatoms, molecules and metal particles. It provides an introduction into the nucleation and growth of oxide layers on single-crystalline metal substrates, putting special emphasis on the various relaxation mechanisms of the oxide lattice to release the misfit-strain with the support. Additionally, the peculiarities of polar oxide films are discussed. In the second part, the different interaction schemes between oxide surfaces and adsorbates are presented from the theoretical point of view as well as on the basis of key-experiment performed with the STM. The focus lies hereby on charge-mediated binding schemes, leading to the formation of cationic or anionic species on the oxide surface. Furthermore, the role of point and line defects for the oxide adsorption behavior is introduced. The potential of thin oxide films as systems with tunable physical and chemical properties is highlighted at the end of this review.
\end{abstract}




\section{List of contents:}

\section{Introduction}

2. Scanning tunneling microscopy on oxide surfaces

4

11

2.1. STM on insulating films 12

2.2. STM on oxide-supported metal particles 18

2.3. Elastic tunneling spectroscopy

2.4. Inelastic tunneling spectroscopy 20

2.5. Photon emission spectroscopy with the STM

\section{Oxide thin films and their surface}

3.1. The metal-oxide interface: Structural aspects $\quad$------------------- 33

3.2. The metal-oxide interface: Electronic aspects $\quad$-------------------- 34

3.3. Thin oxide films: Phase diagrams and terminations $\quad$-------------------- 42

3.4. Growth and relaxation of oxide thin films $\quad$-------------------- $\mathbf{4 8}$

3.5. Defects in oxide films $\quad$-------------------- $\mathbf{5 1}$

3.6. Polar oxide films $\quad$-------------------- $\mathbf{6 0}$

\section{Adsorption on oxide surfaces}

4.1. Adsorption on bulk oxides - Theory $\quad$------------------- 72

4.1.1. Stoichiometric surfaces $\quad$-------------------- 73

4.1.2. Defective surfaces $\quad$ - -------------------- $\quad 75$

4.2. Adsorption on bulk oxides - Experiments $\quad 77$

4.3 Adsorption on thin oxide films - Theory $\quad$--------------------- $\mathbf{8 4}$

4.4 Adsorption on thin oxide films - Experiments $\quad$ -

4.4.1. Charge-mediated adsorption schemes $\quad$ -

4.4.2. Adsorption on polar oxide films $\quad$--------------------- 112

4.4.3. Adsorption on structural peculiarities of thin oxide films $\quad$------------------ $\quad \mathbf{1 1 9}$

4.4.4. Adsorption on defects in oxide thin films $\quad 129$

5. Summary and Outlook 139

6. List of References 142 


\section{Introduction}

Oxide materials are in the focus of applied and fundamental research already for several decades. This unbroken interest is fed by the enormous importance of oxides in almost every section of the economic world. Oxides are widespread as robust and durable materials thanks to their chemical inertness, excellent temperature stability, high electrical resistivity and mechanical hardness. They play a pivotal role in the material sciences, where natural oxides that spontaneously develop on many metal surfaces are exploited as effective and cheap protection layers against corrosion and environmental influences. ${ }^{1,2}$ With the invention of the field-effect transistor, oxides have revolutionized the field of microelectronics. ${ }^{3}$ The ongoing efforts to downscale electronic devices have triggered an intensive search for new oxide materials with superior dielectric properties and high spatial homogeneity. ${ }^{4}$ Furthermore, oxides are the most important support material in heterogeneous catalysis, where they are used to stabilize small metal particles as the chemically active species. ${ }^{5}$ In most catalytic applications, oxides do not only act as passive substrates, but actively influence the chemical performance of the catalyst. They participate in the catalytic cycle by storing and releasing oxygen, ${ }^{6}$ capturing adsorbates on the catalyst surface (spillover effect), ${ }^{7,8}$ exchanging charges with the active component, ${ }^{9,10}$ and maintaining the high dispersion of metal deposits at reaction conditions. ${ }^{11,12}$ Furthermore, oxides are found as key ingredients in chemical sensors, data-storage devices, superconducting elements, photo-active materials and solar cells. 13,14,15,16 But even this list of technologically relevant applications can only give a glimpse on the overall significance of oxide materials.

Their economic importance is certainly one of the driving forces for the intense research activities in the field of oxide materials. The focus lies hereby on the development of new oxide compounds with versatile properties that can be adapted to different technological requirements. Apart from this, oxide materials exhibit an amazing richness and diversity of physical and chemical properties, which makes them extremely fascinating for the fundamental sciences too. The list of phenomena that can be studied on oxide samples is long and includes unusual crystal structures, complex optical, electronic and magnetic properties, a rich phase-diagram, and the occurrence of metal-insulator transitions as well as type-II superconductivity. While the bulk properties, especially of binary oxide systems, are rather well understood, ${ }^{17,18}$ the situation is less satisfying what the physics and chemistry on oxide surfaces concerns. ${ }^{19}$ In contrast to the first impression, the surface properties of oxides are 
much more involved than those of metal and semiconductor samples, despite of the high degree of bond saturation and the chemical inertness of many oxide materials. The complex situation encountered on oxide surfaces has several aspects (Fig.1.1).

(i) The absence of free electrons especially in wide-gap materials reduces the ability to screen structural defects and, in turn, increases their impact on the electronic and chemical properties of the surface. $^{20,21,22}$ Structural defects in oxides comprise a whole zoo of non-stoichiometric atom arrangements and low-coordinated lattice sites, e.g. point defects, interstitial atoms , step edges, kink and corner sites. The most prominent oxide defects are oxygen vacancies, ${ }^{21,23}$ also termed color or $F$ centers due to their effect on the optical properties of the oxide material. ${ }^{24}$ Each defect type is accompanied with a perturbation of the local electronic structure, producing for instance discrete states in the oxide band gap or regions with a modified surface potential, which further increases the inhomogeneity on oxide surfaces.

(ii) Oxides usually exhibit a large variety of surface configurations and terminations that develop on one and the same bulk structure. ${ }^{25}$ Only in few examples, the actual surface corresponds to a cut through the unperturbed bulk lattice, while in most cases the atom positions rearrange at the surface in order to minimize the Gibbs free energy. The resulting surface composition and stoichiometry depends on the cut direction through the bulk crystal, and hence on intrinsic lattice parameters as the number of dangling bonds. However, also environmental effects, such as the oxygen partial pressure and the presence of residual gases during surface formation, play an important role. ${ }^{26,27}$ The surface should hereby reflect the thermodynamic equilibrium situation between the bulk oxide and the surrounding gas phase, although the formation of the lowest-energy configuration might be kinetically hindered. In general, oxide surfaces are either metal or oxygen terminated ${ }^{28,29}$ or covered by a mixed metal-oxygen layer, however many complex surface reconstructions do not fit into this simple scheme. ${ }^{30,31}$ A particular stable surface configuration is achieved by hydroxylation, forming a dense layer of surface $\mathrm{OH}$-groups upon hydrogen or water adsorption. ${ }^{32}$ Whereas most simple-metal oxides (e.g. MgO) develop only a limited number of surface structures, reflecting the fixed stoichiometry of the underlying bulk crystal, the situation can be rather complex for oxides made of multivalent transition metals, such as $\mathrm{Fe}^{28}{ }^{28} \mathrm{~V},{ }^{29}$ or $\mathrm{Ce}$. In such systems, even different oxide phases may coexist at the surface, although only a single phase is stable in the bulk crystal at the experimental conditions. ${ }^{19}$

(iii) Especially in the case of ionic oxides, the surface might be subject to strong relaxation and reconstruction processes, which originate from the perturbation of the delicate balance 
between cations and anions in the bulk crystal. ${ }^{33}$ The distortion of the charge equilibrium at the surface becomes manifest in a large deviation of the surface Madelung potential from the bulk value. While charge-neutral surfaces with a balanced number of metal and oxygen ions (e.g. rocksalt (001) planes) respond to this perturbation only with a slight buckling, strong reconstructions and even thermodynamic instabilities are revealed for polar surfaces consisting of positively and negatively charged layers stacked on-top of each other. For example, the top-layer of (0001) oriented corundum-type oxides experiences an inward relaxation of more than $50 \%$ with respect to the bulk lattice before depolarization takes place (e.g. $-51 \%$ for $\mathrm{Al}_{2} \mathrm{O}_{3}$ and $-60 \%$ for $\left.\mathrm{Cr}_{2} \mathrm{O}_{3}\right){ }^{20}$ The situation is even more dramatic for the rocksalt (111) surface that is unsusceptible to relaxations due to a rigid crystal structure. ${ }^{30}$ As a consequence, polar surfaces of rocksalt-type oxides are thermodynamically unstable and characterized by a diverging electrostatic energy. ${ }^{33}$ However, the oxide polarity might be healed via different mechanisms, for instance by surface restructuring, ${ }^{34}$ adsorption of charged ad-species (e.g. $\mathrm{H}^{+}$ions) ${ }^{32}$ or surface metallization. ${ }^{35}$ In all these examples, the stabilization of the polar surface will again result in a substantial modification of the bulk-truncated structure. This short overview shall provide an impression on the complexity of the structural and electronic properties of pristine oxide surfaces. A detailed discussion on this topic, both from the theoretical and experimental point of view, can be found in Chapter $\underline{3}$.

\section{Polarity Termination Adsorption Growth}

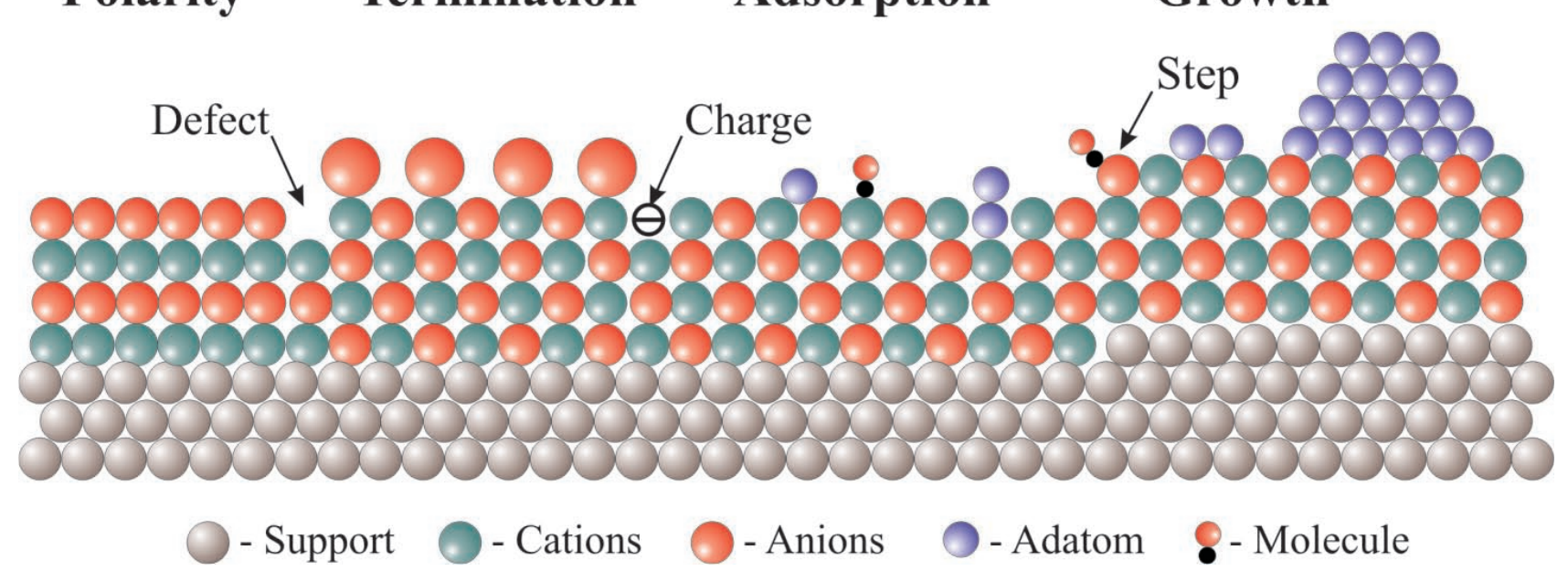

Fig. 1.1: Sketch of an oxide thin film on a metal support, demonstrating important mechanisms and processes to be accounted for when describing the surface properties of the system. 
An even more involved situation is encountered, when adsorption is permitted to reduce the total energy of an oxide surface (Fig. 1.1). ${ }^{36}$ Due to the highly-ionic character of many oxides, adsorption at their surfaces is often governed by electrostatic interactions. ${ }^{19}$ The preferred binding site, being either on a surface anion or a cation, is hereby dictated by the electropositive or electronegative nature of the ad-species. Not surprisingly, adsorption on ionic oxides often involves a charge exchange between adsorbate and support in order to strengthen the Coulomb interaction. The formation of a true chemical bond, on the other hand, is reserved to oxides with strong covalent character and semiconducting or even metallic properties. The spatial distribution of adsorbates and their tendency to form islands on the surface is governed by the balance between adsorbate-adsorbate versus adsorbate-support interactions. Due to the low binding potential of typical oxide surfaces, the three-dimensional Vollmer Weber mode is the dominating growth mode for metals, ${ }^{37}$ while weakly-bound molecular species arrange themselves into open ad-structures. ${ }^{36}$ The adsorption characteristics of oxide surfaces cannot be understood without taking the decisive role of defects into account. $^{21,22}$ In the majority of cases, defects offer a more attractive binding environment than regular lattice sites, reflecting their chemically unsaturated nature. Various examples for the rich adsorption behavior of oxide surfaces will be given in Chapter $\underline{4}$.

Adsorption can be treated from a static or a dynamic point of view. In the static picture, the identification of preferred binding sites and the underlying interaction mechanism is sufficient to describe the adsorption properties of the oxide. In this case, only small modifications of the initial surface configuration are considered. In the dynamic approach, diffusion, dissociation and intermixing processes of adsorbates, as well as a considerable surface reorganization need to be included. ${ }^{38}$ Especially at high temperatures, the adsorption might initiate a substantial mass transport on the surface, inducing the formation of a completely new surface morphology. ${ }^{39}$ This review clearly adopts the static picture, as low-temperature scanning tunneling microscopy (STM) as the major experimental technique employed here has undisputed strengths in the exploration of stationary surface configurations.

The understanding of adsorption properties of oxide materials has reached a high level what the theoretical description of the different binding phenomena concerns. A large amount of literature is available on this topic, especially for $\mathrm{MgO}$ and $\mathrm{Al}_{2} \mathrm{O}_{3}$ as typical simple oxides and $\mathrm{TiO}_{2}$ as an example for a transition-metal compound (see Ref. 304 and following). A short introduction into the different theoretical concepts dealing with adsorption on oxide surfaces 
is given in Chapter $\underline{4.1}$. The large progress on the theoretical side is in contrast to a less satisfactory experimental situation. Although the total number of experiments is huge, most of them are unable to provide atomic-scale insights into adsorption processes on the oxide surface. The situation reflects not only the complex nature of pristine and adsorbate-covered surfaces, as discussed before, but originates from systematic experimental limitations in the exploration of oxide materials. The biggest challenge is the insulating nature of many bulk oxides, which prohibits the application of surface science techniques based on the interaction of matter with electrons or ions. Out of the large arsenal of surface-sensitive techniques, only optical methods and force microscopy are in principle suited to deal with bulk insulators, while most electron-based techniques face severe charging problems. Doping, as a wellestablished approach to introduce conductivity in semiconductors, can be employed only for a limited number of narrow-gap oxides, such as $\mathrm{TiO}_{2}{ }^{40}$ and $\mathrm{ZnO},{ }^{41}$ but does not work for most wide-gap insulators. Even if sufficient bulk conductivity is attainable, the doping approach bears a number of disadvantages, as the required defect concentration will also affected the surface properties. A prominent example for this dilemma is $\mathrm{TiO}_{2}$, where the insertion of oxygen vacancies to install bulk conductivity gives rise to a finite defect concentration also at the surface, ${ }^{42}$ which in turn influences the adsorption behavior. ${ }^{43,44}$ For many conductive oxides, on the other hand, fabrication of stoichiometric, crystalline and atomically-flat surfaces is an intricate task, as the desired surface is often unstable against phase transitions and reconstructions. Especially for transition-metal oxides with several phases coexisting in a narrow window of the phase diagram, the preparation of defect-poor and well-defined samples is difficult.

An elegant way to overcome charging problems associated with the use of wide-gap insulators is the growth of oxides in the form of thin films on metal or semiconductor supports. 45,46,47,48,49 A genuine oxide film is hereby distinguished from a chemisorbed oxygen layer by the condition that the free-energy of the oxide phase is lower than the chemical potential of the adsorbed $\mathrm{O}$ atoms on the metal surface. ${ }^{50}$ As the free-energy of a thin oxide film includes two extra terms, accounting for the metal-oxide adhesion and the surface energy, the formation criteria for thin-film and bulk oxides are different. When choosing the right metal substrate, high quality and defect poor oxide films can be grown for a large variety of materials. Common recipes for thin film preparation are (i) oxidation of a suitable metal single crystal, (ii) reactive deposition of metal atoms in an oxygen atmosphere or (iii) growing a 
metal film followed by post-oxidation at high temperature (Fig. 1.2). To improve the availability of oxygen during film growth, the support might be loaded with oxygen prior to the actual preparation step, e.g. by implanting $\mathrm{O}$ atoms or forming an O-rich precursor state. In most cases, the as-prepared film needs to be thermally treated in order to improve its crystallinity and lower the defect concentration.

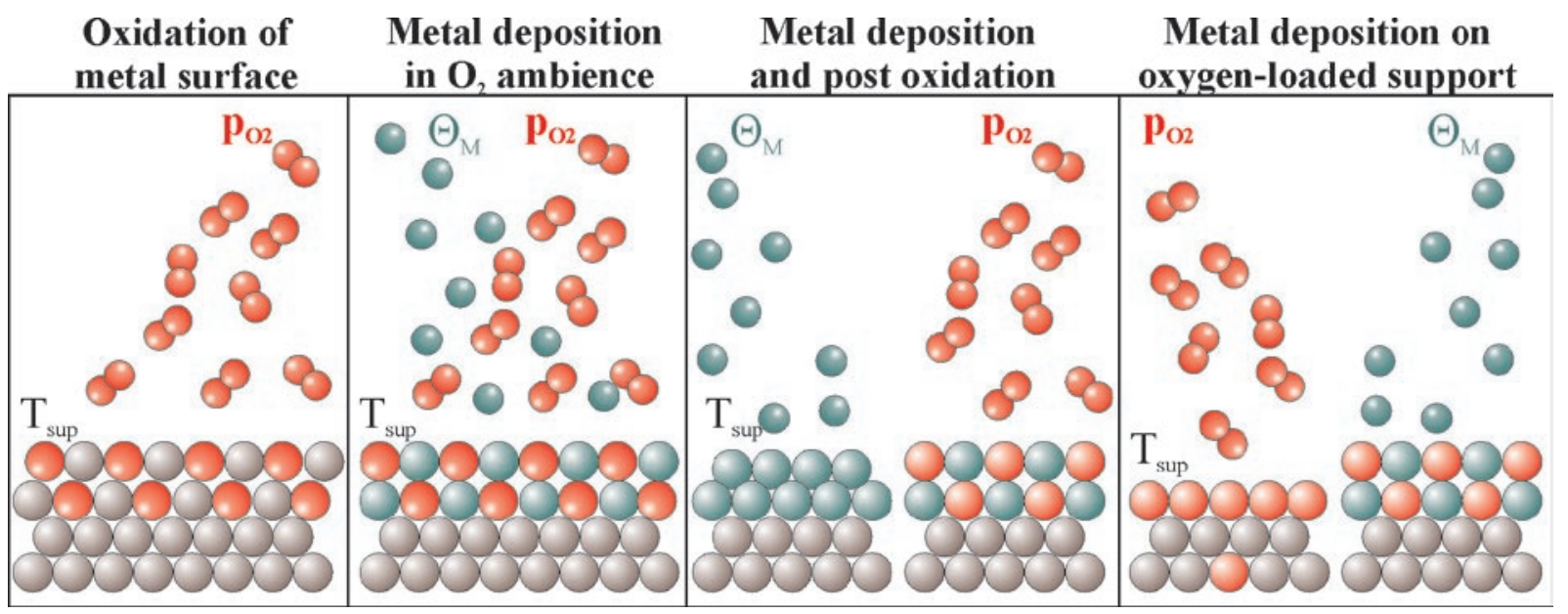

Fig.1.2: Common approaches to prepare thin oxide films on metal supports. The critical preparation parameters are the oxygen partial pressure $p_{O 2}$, the metal exposure $\Theta_{M}$ and the temperature of the support $T_{\text {sup }}$ during deposition and thermal treatment.

The permeability of thin oxide films for electrons relies on tunneling or ballistic transport, both depending on the film thickness, the energy of the injected electrons and the size of the oxide band-gap. For wide-gap materials, such as $\mathrm{MgO}$ and alumina, films of up to 50 monolayer (ML) thickness can be investigated with typical surface science techniques including STM. ${ }^{51}$ For narrow gap-materials, such as $\mathrm{V}_{2} \mathrm{O}_{3}$ and $\mathrm{Fe}_{3} \mathrm{O}_{4}$, films of almost arbitrarily thickness are accessible. In both cases, bulk-like properties emerge in the top-most oxide layers and the influence of the metal support on the adsorption behavior becomes negligible. Consequently, oxide films of more than a dozen ML thickness represent suitable model systems to study the properties of the corresponding bulk material. ${ }^{49,52}$

For ultra-thin films, on the other hand, the metal-oxide interaction may interfere considerably with the intrinsic behavior of the oxide material. This interplay usually causes the emergence of new, film-specific properties that originate from the residual influence of the metal support. Ultra-thin oxide films can thus be considered as a new class of composite materials, whose characteristics can be modified by choosing suitable metal-oxide combinations and changing the film thickness. Also the chemical composition and defect concentration of oxide thin films can be varied over a wide range, for instance by preparing the film in more oxidizing or 
reducing environments, introducing defects via sputtering and electron bombardment, or inserting dopants with given quantities. In contrast to bulk oxides, the film composition does not have to be in equilibrium with the respective bulk stoichiometry and can be varied in a much broader window. The use of thin oxide films grown on metal supports is therefore not only advantageous to explore oxide materials themselves, but opens the possibility to fabricate a new class of tunable compounds with yet unexplored properties. ${ }^{53}$

The following article reviews the current state-of-the-art experiments on oxide materials and their adsorption properties, connecting the experimental results to recent theoretical developments in the field. Whereas the focus lies on the investigations of thin oxide films, comparison to bulk materials are made at several points. The amount of data on this topic has increased dramatically in the past decade and can hardly be summarized within one article. The author therefore takes a 'local view' on this topic, discussing primarily experiments performed with scanning tunneling microscopy and related spectroscopic techniques. This selection is partly triggered by the experimental expertise of the author, but also emphasizes the advantages of exploring oxide surfaces with a spatially-resolving technique. A short introduction into the principles of STM and its spectroscopic applications is given in the next chapter. Chapter $\underline{3}$ provides an overview of STM experiments on pristine oxide surfaces, whereas Chapter $\underline{4}$ discusses the adsorption of atomic and molecular species as well as the nucleation and growth of metal particles on oxide supports. 


\section{Scanning tunneling microscopy on oxide surfaces}

Due to the absence of charging problems, thin oxide films can be explored with the standard techniques of surface science. ${ }^{54}$ Spatially-averaging structural data is usually obtained by diffraction and scattering methods, e.g. by low-energy-electron diffraction (LEED), photoelectron diffraction (PED and XPD) and grazing-incidence X-ray diffraction (GIXD). Electronic properties are probed with electron and optical spectroscopies, such as ultra-violet (UPS) and X-ray photoelectron spectroscopy (XPS), electron-energy-loss (EELS) and UVVIS spectroscopy. The adsorption behavior of oxide surfaces can be characterized by infraredreflection-absorption (IRAS) and high-resolution electron-energy-loss spectroscopy (HREELS) as well as by temperature-programmed-desorption (TPD) measurements.

Common to all these techniques is their limited spatial resolution and the non-local character of the acquired information. Although the sensitivity of non-local methods is usually high enough to detect small densities of defects and adsorbates, their local environment and spatial arrangement on the surface is not revealed. This limitation interferes with the structural and electronic inhomogeneity of most oxide surfaces that typically exhibit a large number of nonequivalent sites. ${ }^{21,23,55}$ Defects and low-coordinated atoms have a particularly large impact on the properties of real oxide materials, but often produce only weak and inconclusive signatures in spatially averaging methods, especially in reciprocal-space techniques that are sensitive to periodic structures. The shortcoming of non-local approaches becomes even more evident when adsorption from the gas phase is permitted. Even well-prepared oxide surfaces offer a variety of potential adsorption sites, to which adatoms and molecules bind in different conformations and charge states. A deposition of metal particles further increases the complexity of the system. Metal clusters containing a few dozens of atoms exhibit pronounced size-dependent properties, ${ }^{56}$ which are usually washed out in non-local measurements due to the unavoidable size and shape distribution in the particle ensemble. For instance, electron quantization effects in ultra-small clusters ${ }^{57}$ and their size-dependent optical response $^{58}$ are not revealed in ensemble measurements. Analyzing the distinct properties of metal particles on oxide supports is however of pivotal importance for elucidating the structural and electronic preconditions for the catalytic performance of a particle-support system. $^{5}$ 
The application of spatially-resolving experimental techniques is an ideal approach to circumvent these problems. Real-space information at the atomic scale can be provided by scanning probe techniques, in particular by scanning tunneling (STM) and atomic force microscopy (AFM). ${ }^{59,60}$ In contrast to diffraction experiments, both techniques are sensitive to non-periodic structures and therefore suited to study the spatial distribution of defects and adorbates on oxide supports as well as the size and shape of individual metal deposits. The signal generation in AFM is based on force interactions between a local sensor tip and the surface and therefore independent of the sample conductivity. ${ }^{61}$ This enables the investigation of insulating oxides with atomic resolution, as demonstrated for bulk $\mathrm{CeO}_{2},{ }^{62} \mathrm{MgO}^{63}$ and $\alpha$ $\mathrm{Al}_{2} \mathrm{O}_{3}$, ${ }^{64}$ as well as for alumina and magnesia thin films grown on $\mathrm{NiAl}(110), \mathrm{Ni}_{3} \mathrm{Al}(111)$ and Ag(001), respectively. ${ }^{65,66,67}$

The STM, on the other hand, probes the local density of states (LDOS) of the sample surface and therefore its electronic properties. ${ }^{68}$ Consequently, STM is limited to conductive samples, making experiments on thin and electron-transparent oxide films particularly attractive. Apart from topographic imaging, STM supports a large variety of spectroscopic techniques, for example conductance spectroscopy, inelastic-electron-tunneling spectroscopy (IETS) and light-emission spectroscopy. Using these additional capabilities of STM, also the electronic, vibrational and optical properties of the sample surface can be probed on a nanometer scale. The next paragraph gives a short introduction into the principles of STM and embedded spectroscopic techniques, and discusses general aspects of their application to oxide films.

\subsection{STM on insulating oxide films}

A detailed introduction into scanning tunneling microscopy can be found in several textbooks and is beyond the scope of this paper. ${ }^{59,60}$ In short, STM images obtained in the constantcurrent mode reflect the topographic and electronic properties of the sample convolved with the tip characteristic, as first shown by Bardeen ${ }^{69}$ and Tersoff / Hamann ${ }^{70}$. For the sake of simplicity, the interrelation between the tip-sample parameters and the tunnel current is described here with the semi-classical Wentzel-Kramers-Brillouin approximation. ${ }^{71}$ The electron current $I$ at the tip position $r_{\text {Tip }}$ is calculated from the tunneling probability between two planar electrodes at zero temperature, integrated over the energy range where pairs of filled-initial and empty-final states are available in tip and sample (elastic tunneling): 
Eq. $1 \quad I \propto \int_{E_{F}}^{E_{F}+e V} d E \rho_{s}\left(r_{\text {Tip }}, E\right) \rho_{t}(E-e V) T(z, E, V)$

The tunneling probability at certain energy $E$ depends on the local state density in tip $\rho_{t}$ and sample $\rho_{s}$, weighted by the transmission probability $T$ of electrons through the trapezoidal vacuum barrier. The barrier geometry is hereby fixed by the tip-sample distance $z$, the applied sample bias $V$ and the work function average of both electrodes: $\phi=1 / 2\left(\phi_{t}+\phi_{s}\right)$ (Fig. 2.1):

Eq. $2 \quad T(z, E, V)=\exp \left[-\frac{2 z}{\hbar} \sqrt{2 m(\phi-E+1 / 2 e V)}\right]$

The integral in Eq. 1 runs from the tip to the sample Fermi level $\left(E_{F}\right)$ and includes all filledempty state pairs in the emitter and the acceptor, respectively, in the energy window given by the bias voltage. However, as the tunneling probability depends exponentially on the barrier height and thus on the initial electron energy with respect to the vacuum level, electronic states near the Fermi level of the emitter contribute strongest to the tunnel current (Fig. 2.1). In most applications, STM images are obtained by adjusting the tip-sample distance $z$ in a way that the tunnel current is kept constant. The resulting tip-height images are consequently no one-to-one representation of the sample topography, but include spatial modulations of the sample electronic structure as well.

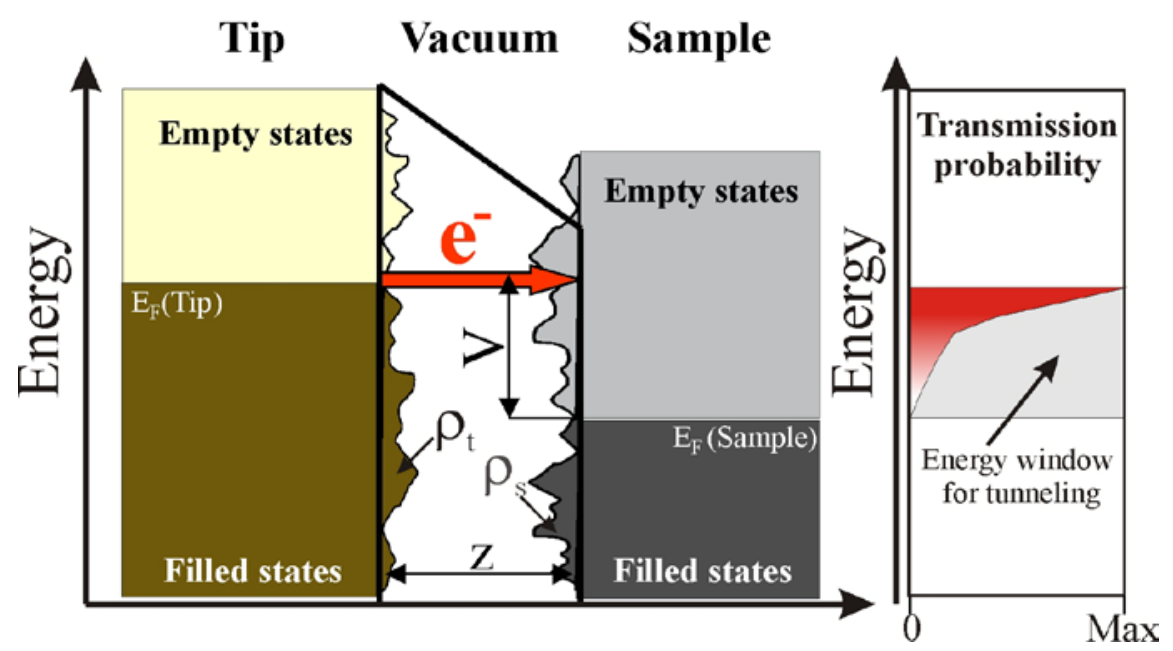

Fig. 2.1: Potential diagram of an STM junction (right) and sketch of the energy-dependent tunneling probability across the tip-sample gap (left).

The requirement that filled and empty states need to be available for tunneling transport has a number of consequences for the investigation of oxide materials with the STM. ${ }^{72}$ Many oxides are characterized by a band gap around the Fermi level, inhibiting tunnel processes at low bias voltages. Even when the sample bias is higher (lower) than the conduction (valence) band onset in the insulator and electron (hole) injection is possible from an energetic point of view, the small mobility of carriers in the oxide bands might prevent efficient electron transport. 
STM experiments even on thick oxide films are, however, possible when one of the following conditions is fulfilled:

- The oxide has a sufficient density of intrinsic carriers even at low temperature. This is the case for many transition-metal oxides with narrow band gaps, e.g. for $\mathrm{V}_{2} \mathrm{O}_{3}, \mathrm{Fe}_{3} \mathrm{O}_{4}$ and $\mathrm{ReO}_{3}$. ${ }^{18}$

- Electron transport through the oxide films is possible via tunneling, as the wave functions of the metal support sufficiently penetrate the oxide layer to overlap with the tip electronic states. In this case, the STM junction can be stabilized even at low bias. However, the conductance mechanism is strictly limited to ultra-thin insulating films of 5-10 A thickness.

- Electrons are injected ballistically into the oxide conduction band and propagate towards the metal support in the tip-induced electric field. The development of quantized states in the oxide band above the vacuum energy (so called Gundlach or field-emission resonances) ${ }^{73,74}$ strongly increases the maximum transport length in this case. As a result, insulting films of up to $50 \AA$ thickness can be studied when operating the STM in the near field-emission regime (5-15 V), however on the expense of the achievable spatial resolution.

- Sufficient oxide conductivity is established at elevated temperatures, when a small number of valence electrons is excited into the conduction band and provides free carriers for transport. The possibility to perform STM experiments at high temperature has been demonstrated for several wide-gap insulators, such as for $\mathrm{NiO}(500 \mathrm{~K})^{75}$ and $\mathrm{CeO}_{2}(700 \mathrm{~K}){ }^{76}$

- A finite carrier concentration is adjusted in the oxide bands via doping with intrinsic defects (e.g. oxygen vacancies) or impurity atoms. This approach is commonly used for semiconductors and can be employed for narrow-gap oxides as well. In the case of $\mathrm{TiO}_{2}$ or tin-oxide for example, ${ }^{40}$ the creation of oxygen vacancies produces a large number of donor states in the band gap that are able to release electrons into the conduction band. For wide-gap materials, such as $\mathrm{MgO}$, doping does not lead to a significant increase of the carrier concentration.

- Conductive surface states might be formed on certain oxides upon adsorption of molecular species. The most prominent example for this mechanism is the metallization of the $\mathrm{ZnO}(10-10)$ surface after hydrogen exposure. ${ }^{35}$

Even if tunneling through the oxide material is feasible for one of the given reasons, the tunneling characteristic will follow different rules than on metal samples. ${ }^{72}$ In particular, the contribution of inhomogeneous and spatially-varying electronic properties might be larger. This is especially true for tunneling through the oxide band gap, as regular electronic states are absent and defects might dominate the transport behavior. ${ }^{77}$ In low-bias STM images of thin oxide films, the apparent morphology might be completely governed by properties of the metal-oxide interface, accommodating the required electronic states for tunneling. ${ }^{78}$ For 
example, atomically-resolved STM images have been obtained from the interface between a thin alumina film and the $\mathrm{NiAl}(110)$ support, although the respective atomic layer is buried below three other oxide planes. ${ }^{31}$ At bias voltages close to the onset of the conduction or valence band, a new contrast might develop in the STM images, which has no topographic origin but arise from the sudden availability of the oxide states for electron transport. This contrast change is often accompanied by a drastic increase of the apparent film height, which can be exploited to estimate the gap size of the oxide material. ${ }^{79,80,81}$ Even for bias voltages well beyond the band onsets, the actual image contrast might not be governed by topographic effects. In fact, local changes in the electron transmission probability $T$ through the oxide film start to play a decisive role. At high voltages, $T$ will be governed by electron transport through confined vacuum states (more precisely through field-emission resonances) ${ }^{73,74}$ and not by the tunneling probability any more. As the availability of those transport channels depends on the work function, a new contrast mechanism becomes active that reflects local work function modulations in the oxide film instead of the real surface morphology (Fig. 2.2). ${ }^{82}$ See Chapter $\underline{3.6}$ for more details.

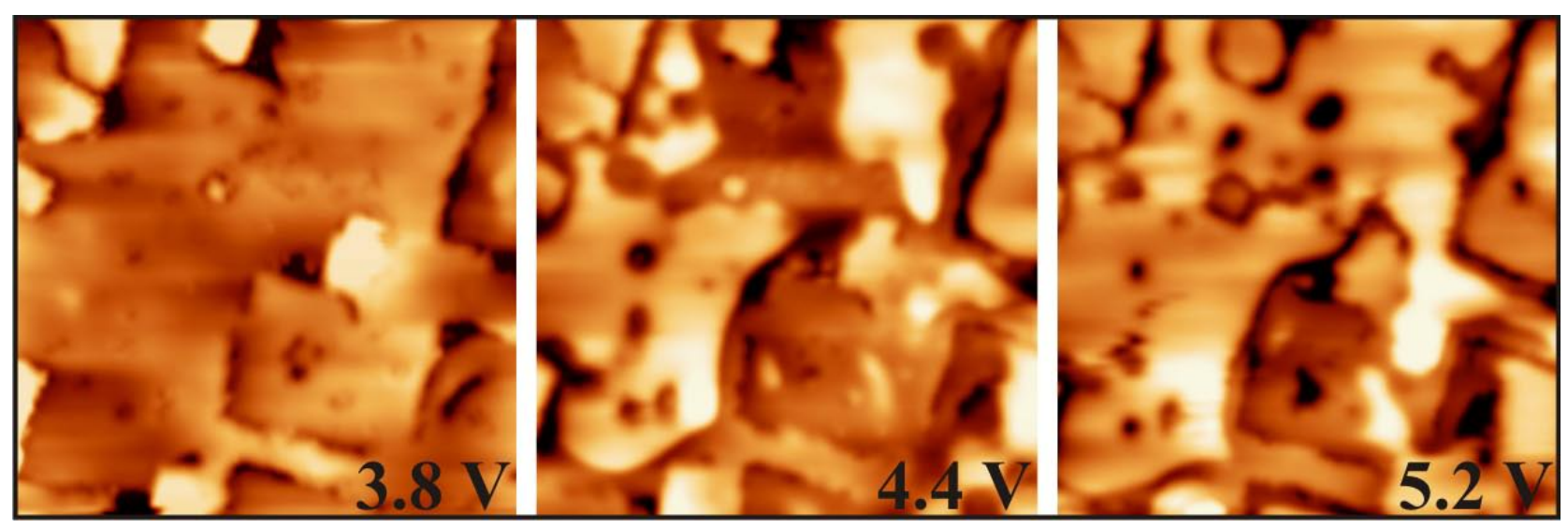

Fig. 2.2: STM images of an identical surface region of an $8 \mathrm{ML} \mathrm{MgO/Mo(001)} \mathrm{film} \mathrm{taken} \mathrm{at} \mathrm{different}$ sample bias $\left(35 \times 35 \mathrm{~nm}^{2}\right)$. Only for tunneling slightly above the conduction band onset, a representation of the surface structure is revealed (left), whereas at higher voltages, a structureindependent mechanism dominates the image-contrast that is governed by the availability of fieldemission resonances for electron transport. ${ }^{82}$

In general, STM imaging of oxide films is subject to large bias-dependent contrast changes, which renders an analysis of the true surface morphology difficult. It is therefore important to reiterate that especially on insulating surfaces, STM measurements are by no means representations of the actual morphology, but affected by electronic contributions to the transport characteristic. 
A further difference with respect to metal samples concerns the effect of voltage division in a tip-sample junction containing an insulating film. ${ }^{83,84}$ Due to the small electron density, oxides are often unable to screen the tip-electric field directly at their surface. As a consequence, a fraction of the applied junction bias drops inside the oxide film, leading to a bending of the oxide bands (Fig. 2.3A). ${ }^{85}$ The amount of band bending in the tip-electric field depends on the thickness $d$ and the dielectric constant $\varepsilon_{\mathrm{r}}$ of the oxide material. It can be estimated by modeling the tip-sample junction as a plate capacitor that is partly filled with a dielectric medium. The ratio between the voltage drop inside the oxide film and the total junction bias is given by: $\eta=\frac{d}{z \varepsilon_{r}}$ with $z$ being the tip-surface distance. As a result of this bias division, the band onsets in a thin oxide film shift away from their flat-band position $E_{o}$ by: $\Delta E=E_{0}\left(\frac{\eta}{1-\eta}\right)$ (Fig. 2.3). ${ }^{83}$

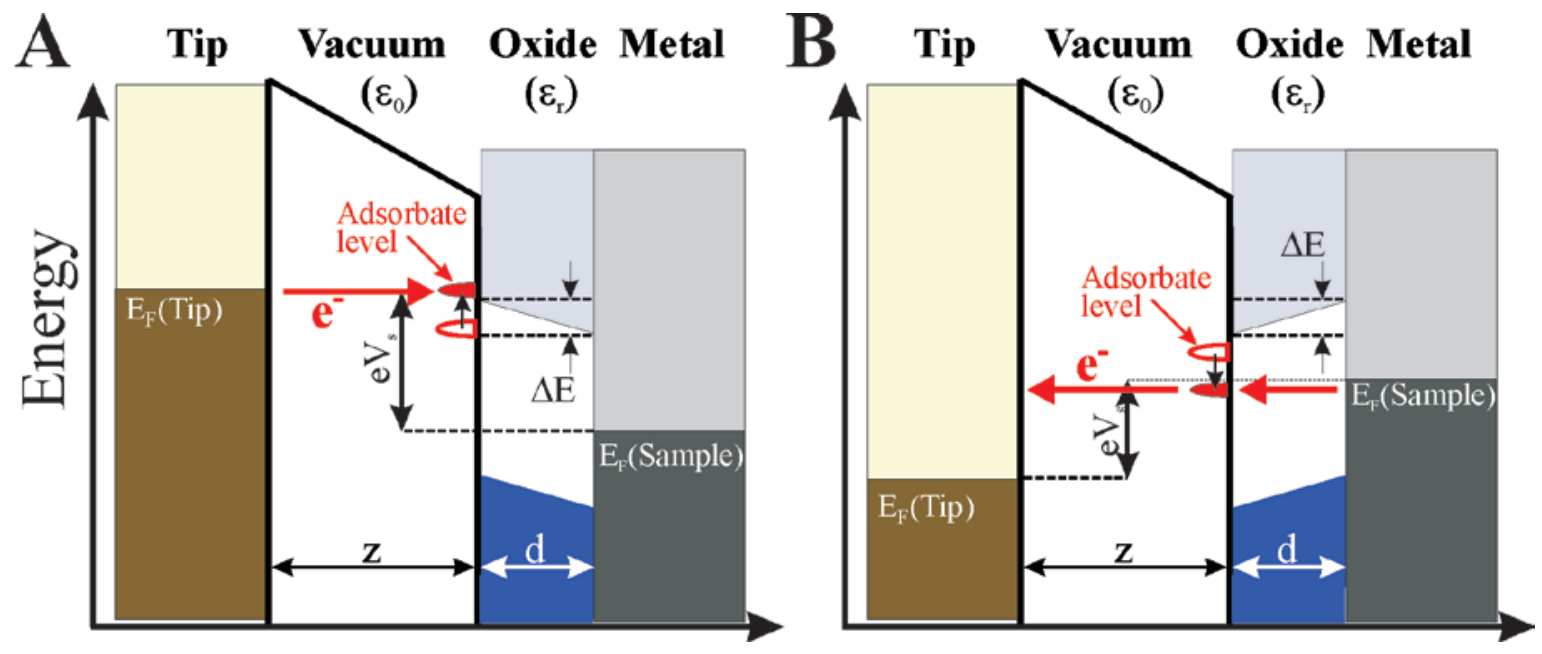

Fig. 2.3: (A) Potential diagram of an STM junction containing an insulating film. Due to the tipinduced field, the bands of the insulator but also the energy levels of an adsorbate shift to higher energies with increasing positive sample bias and to lower energies at negative voltage. (B) Low-lying energy levels of an adsorbate might now cross the Fermi level of the system, resulting in tip-induced charging of the ad-species. In this case, the same electronic state becomes available for tunneling at positive (not shown) and negative bias voltage, a phenomenon known as bipolar tunneling.

Tip-induced band bending also affects the position of energy levels in adsorbates bound to the oxide surface. The adsorbate electronic structure either adjusts to the band edge position or to the local vacuum energy and is therefore subject to similar energy shifts as the oxide bands in the tip-electric field (Fig. 2.3). ${ }^{86}$ Only when the metal substrate below the oxide film serves as a reference for the adsorbate electronic structure, the influence of the electric field in the STM junction becomes negligible. In particular cases, the tip-electric field might be responsible for an electronic state to cross the Fermi level of the metal-oxide system (Fig. 2.3B). In this 
scenario, the adsorbate becomes temporally charged in the biased STM junction. ${ }^{84,87,88}$ Hereby negative charging occurs at negative sample bias, as a formerly unoccupied state is pushed below the Fermi energy, while a positive voltage stimulates the emptying of an originally filled state. The sample bias $V_{c}$ at which level crossing occurs depends inversely on the amount of band bending, according to: $e V_{c}=-E_{0}\left(\frac{1+\eta}{\eta}\right)$. Due to the small screening response of typical oxides, the crossing bias is much more sensitive to the tip-electric field than the energy position of the respective state in the direct (resonant) tunneling mode (Fig. 2.4). The effect of level crossing and the subsequent charging of the adsorbate are limited to ad-species with low-lying electronic states bound to weakly polarizable, dielectric films. The related phenomenon that one and the same electronic state contributes to the electron transport at positive as well as negative bias is generally referred to as bipolar tunneling. ${ }^{83}$
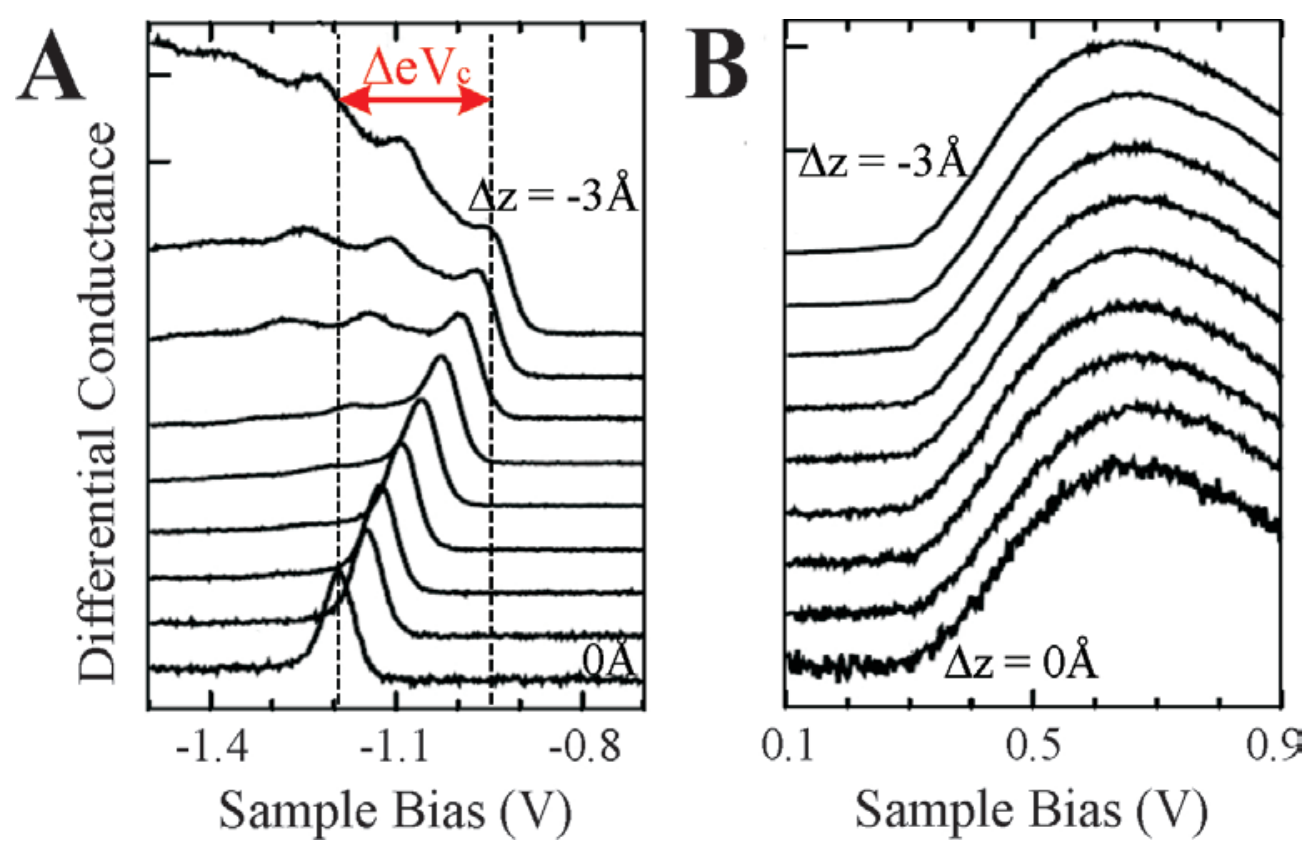

Fig. 2.4: Bipolar tunneling through the LUMO of a CuPc molecule adsorbed on a thin alumina / NiAl film. (B) Direct tunneling, where electrons propagate resonantly from the tip through the adsorbate level into empty states of the metal support (see scheme in Fig. 2.3A). Due to the small oxide thickness, the influence of the tip-electric field on the peak position is small in this case. (A) The same state contributes to the electron transport at negative bias, as it is pushed below the Fermi level of the $\mathrm{NiAl}$ in the tip electric field (see Fig. 2.3 B). In this scenario, the electrons tunnel into the tip from the now-occupied adsorbate state that is refilled with electrons from the metal in a second step. The crossing bias $\mathrm{eV}_{\mathrm{c}}$, at which the state moves below the Fermi level, hereby shifts to lower values when the tip approaches the surface ( $\Delta \mathrm{z}$ becomes more negative) ${ }^{84}$

In general, the dielectric response of an oxide film in a biased STM junction induces a shift of the experimental energy positions of oxide or adsorbate electronic states, whose magnitude is 
given by thickness and dielectric properties of the film. This effect needs to be considered whenever conductance spectroscopy is performed on insulating surfaces with a limited screening ability.

\subsection{STM on oxide-supported metal particles}

In analogy to adsorbates, also metal particles on thin insulating films can change their charge state in response to the applied sample bias. ${ }^{89,90,91,92}$ The electric isolation of the particle between the vacuum barrier on one side and the oxide barrier on the other brings about that its chemical potential floats between the Fermi levels of both electrodes. The charge state of the particle now adjusts to the applied sample bias, to the voltage division in the double barrier junction and the transmission probability of both barriers. In contrast to the molecular case, the charging is not governed by the filling/emptying of discrete energy levels that are pushed above or below the substrate Fermi level, because even small particles have already a quasicontinuous state density. The required energy for a charging event is rather determined by the Coulomb repulsion that needs to be overcome when a single electron is added or removed from the particle electronic system. ${ }^{89,93}$ In the most simple approach, the charging energy $E_{c}$ depends on the inverse capacitance $C$ of the particle according to: $E_{c}=e^{2} / C$, and therefore increases with decreasing particle size. ${ }^{94}$

The Coulomb charging of metal particles on insulating films leads to a characteristic transport behavior through the double-barrier STM junction, which affects the imaging and spectroscopy of metal deposits on oxide layers (Fig. 2.5). ${ }^{95,96}$ At low sample bias, electrons from the tip have not enough energy to overcome the Coulomb repulsion and cannot enter the particle electronic system. As a result, the tunnel current through the particle is suppressed and a zone of zero-conductance shows up, the so called Coulomb blockade region (see Fig. 2.5C). With increasing sample bias, the electron energy reaches the charging energy and the tunnel current sets in, producing a pronounced peak in the differential conductance (dI/dV). The lowest charging peaks are usually symmetric to the zero-bias position, as the Coulomb energies for adding or removing an electron are similar for sufficiently large electronic systems. A small deviation from a symmetric peak configuration provides a first indication for the presence of extra charges that reside on the particle even in the unbiased junction. ${ }^{89}$ For particles with good electric isolation from the tip and sample electrodes, also higher charging peaks become visible in the differential conductance, a behavior known as Coulomb staircase. The charging peak that occurs at $n$-times the single-electron Coulomb energy is hereby 
associated with the $n^{\text {th }}$ excess electron being transferred to (from) the particle (Fig. 2.5C). An experimental example for Coulomb charging in the STM is shown in Fig.2.6 for differentlysized Ag particles grown on a thin alumina/NiAl(110) film. ${ }^{95}$
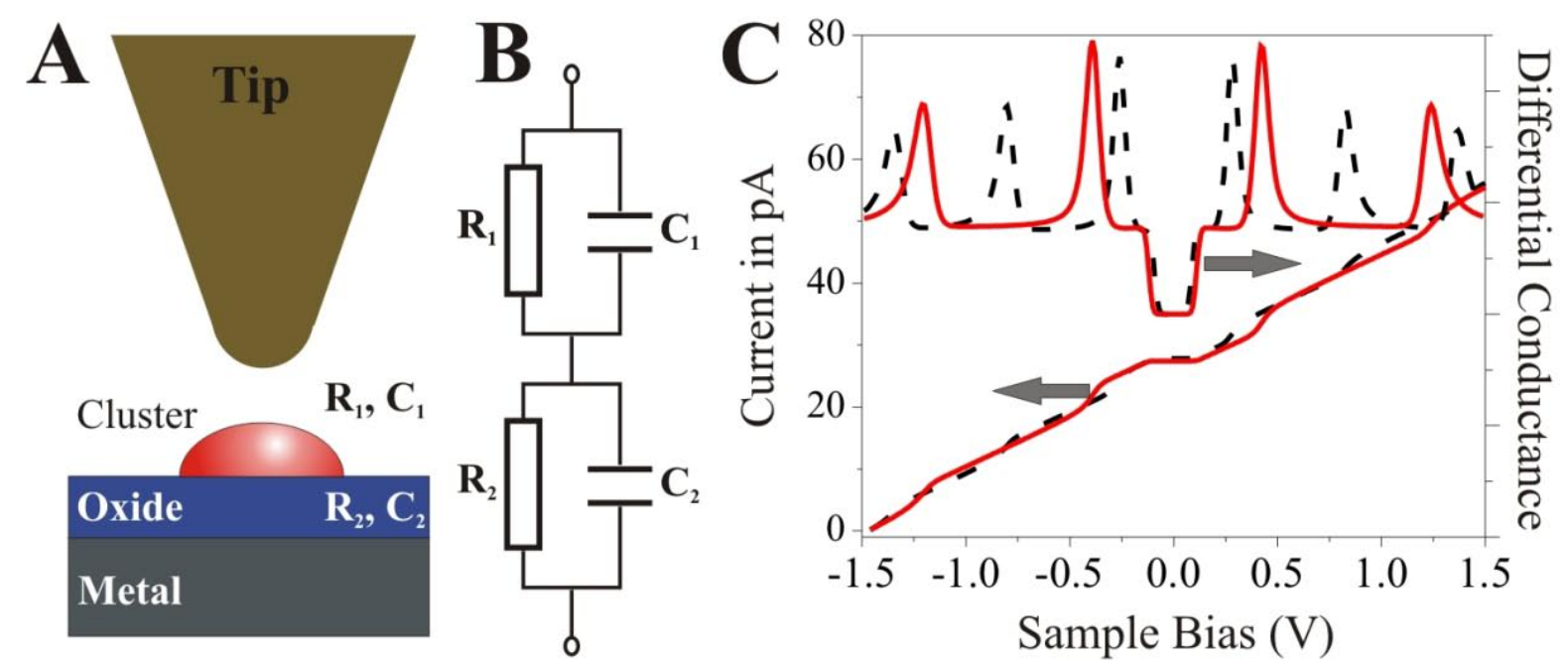

Fig. 2.5: (A) Model geometry and (B) equivalent circuit diagram of a double-barrier STM junction leading to Coulomb charging effects. The vacuum and oxide barrier are each described with a capacitance and a resistance value. ${ }^{89}$ (C) Simulated electron current and conductance behavior through a large (dashed line) and a smaller (solid line) metal particle on an insulating support, calculated with the model given in Ref. 89. The simulation parameters for the vacuum barrier are $\mathrm{R}_{1}=5 \times 10^{-10}$ $\Omega$ and $C_{1}=2 \times 10^{-19} \mathrm{~F}$. The oxide barrier is modeled with $\mathrm{R}_{2}=5 \times 10^{-8} \Omega$ and $\mathrm{C}_{2}=9 \times 10^{-9} \mathrm{~F}$ and $6 \times 10^{-9} \mathrm{~F}$ for the large and the small particle, respectively. The region of suppressed conductance around zero bias and the regular charging steps are clearly resolved in both cases.

The size dependence of the charging energy of single metal particles can be approximated with a spherical capacitor model, relating the Coulomb energy to the particle radius $r$ and the dielectric constant $\varepsilon_{r}$ of the surrounding medium via: $E_{c}=e^{2} /\left(4 \pi \varepsilon_{o} \varepsilon_{r} r\right) .{ }^{94}$ Using this formula, the charging energy of a $1 \mathrm{~nm}$ particle on an alumina support $\left(\varepsilon_{\mathrm{Al} 2 \mathrm{O} 3}=10\right)$ calculates to 730 $\mathrm{meV}$, but decreases to $73 \mathrm{meV}$ when the diameter increases to $10 \mathrm{~nm}$. A differential conductance spectrum of such particle-oxide system would therefore consist of equidistant charging peaks separated by a constant gap of $\Delta V=E_{c} / e=730 \mathrm{mV}(73 \mathrm{mV}$ ) (see Fig. 2.6). In a more sophisticated approach, being developed by Hanna and Tinkham, ${ }^{89}$ the electron transport through a double-barrier junction is modeled by the current flow through two shunted resistor/capacitor elements connected in series (Fig. 2.5B). Whereas the first element $\left(\mathrm{R}_{1}, \mathrm{C}_{1}\right)$ mimics the resistance/capacitance of the tip-particle junction, the second one $\left(R_{2}, C_{2}\right)$ describes the electron transport through the insulating film. This model does not only reproduce the energy position of charging steps, but also predicts absolute conductivity changes on the basis of the tunneling rates through both barriers (see Fig. 2.5C). 


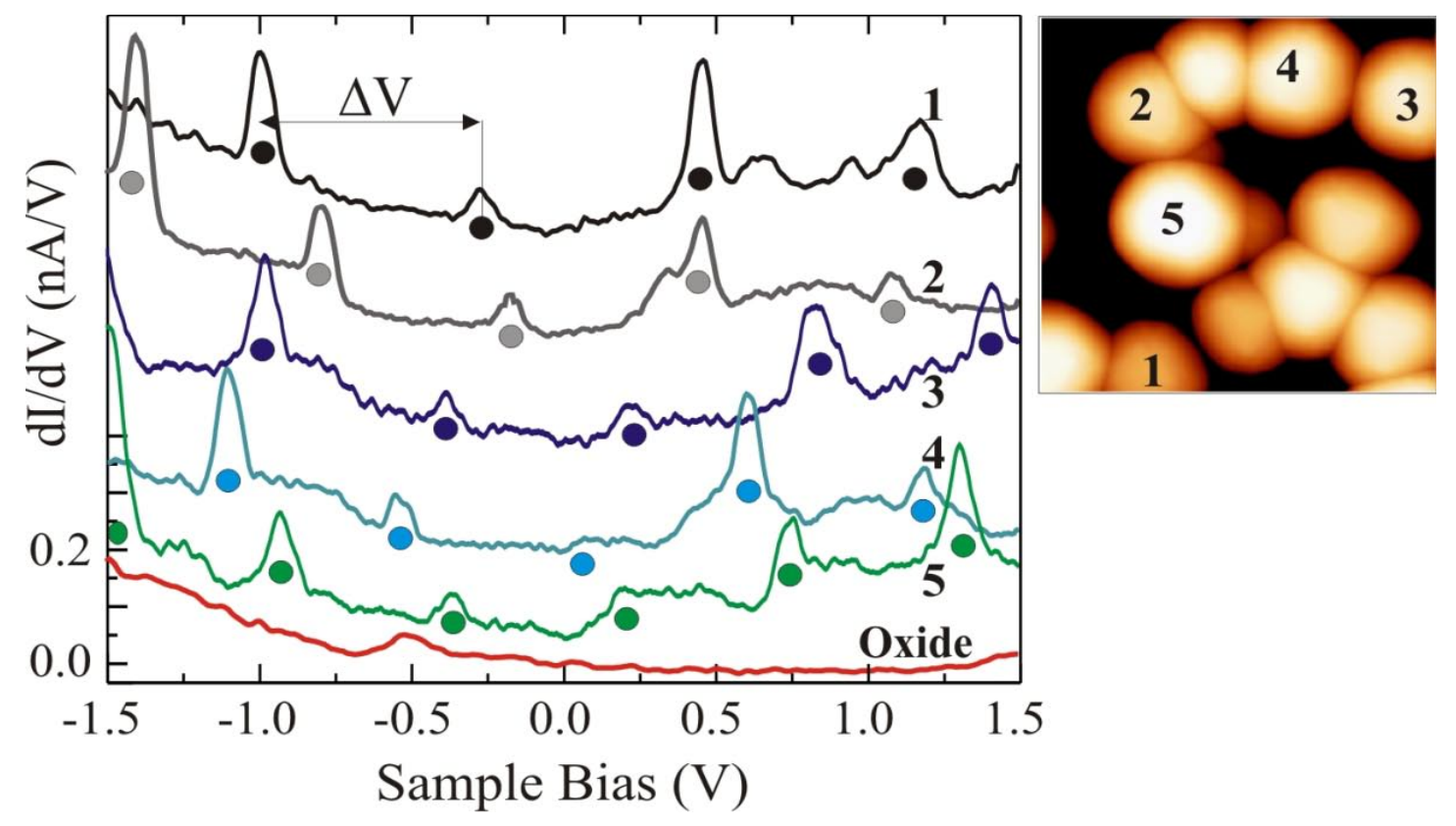

Fig. 2.6: Conductance spectra of differently-sized Ag particles on alumina / $\mathrm{NiAl}(110)$ and the bare oxide, taken at $\mathrm{V}_{\text {Sample }}=1.5 \mathrm{~V}, \mathrm{I}=1.0 \mathrm{nA}$. Equidistant $\mathrm{dI} / \mathrm{dV}$ peaks that represent the Coulomb staircase are marked by small dots. The investigated particles are labeled in the topographic STM image shown in the inset (image size: $50 \times 50 \mathrm{~nm}^{2}$ ). ${ }^{95}$

The distinct transport characteristic of a double-barrier junction needs to be considered in any STM measurement on metal particles deposited on an insulating film. Besides Coulomb charging effects, also a discontinuous and quantized electronic structure of the system might affect the differential conductance of the tunnel junction. Experimental approaches that distinguish both effects and exploit STM conductance measurements to obtain information on the sample electronic-structure are introduced in the next paragraph.

\subsection{Elastic tunneling spectroscopy}

As discussed before, the tunnel current through an STM junction contains always a topographic and an electronic contribution. ${ }^{59,60}$ The electronic data can be separated from the structure part by means of spectroscopic measurements, probing the current response to a linear bias ramp at constant tip-sample distance. By this means, the $z$-dependence of the tunneling probability is disabled, while the LDOS of tip and sample dominates the current change. Whereas the $I-V$ characteristic gives only an integral measure of the state density in the probed energy window, energy resolved information is obtained by detecting the harmonic response of the tunnel current to a bias modulation, an experimental technique termed differential conductance (dI/dV) spectroscopy (Fig. 2.7). The kind of information detected 
with this approach can be determined from calculating the bias derivative of the tunnel current formulated in Eq.1 of Chapter 2.1:

$$
\begin{aligned}
\text { Eq. } 3 \quad \frac{d I}{d V}\left(V_{0}\right) & \propto \rho_{S}\left(r_{T i p}, V_{0}\right) \rho_{T}\left(E_{F}\right) T\left(z, V_{0}\right)+\int_{E_{F}}^{E_{F}+e V} d E \rho_{S}\left(r_{T i p}, E\right) \frac{d \rho_{T}\left(E-e V_{0}\right)}{d V} T\left(z, E, V_{0}\right) \\
& +\int_{E_{F}}^{E_{F}+e V} d E \rho_{S}\left(r_{\text {Tip }}, E\right) \rho_{T}\left(E-e V_{0}\right) \frac{d T\left(z, E, V_{0}\right)}{d V}
\end{aligned}
$$

Using the assumption that the tip LDOS and the transmission coefficient are approximately bias independent in the explored spectral range ( $\mathrm{d} \rho_{T} / d V \sim 0$ and $d T / d V \sim 0$ ), the first term of Eq. 3 will dominate the $\mathrm{dI} / \mathrm{dV}$ signal. The current response to a small bias change therefore provides a measure of the state density in the sample surface probed at the position of the tip and scaled by the transmission coefficient and the tip LDOS. ${ }^{6}$

Eq. $4 \quad \frac{d I}{d V}\left(V_{o}\right) \propto \rho_{S}\left(r_{T i p}, V_{0}\right) \rho_{T}\left(E_{F}\right) T\left(z, V_{0}\right)$

Differences in the $\mathrm{dI} / \mathrm{dV}$ spectra acquired on different surface locations can now be associated to changes in the respective sample electronic structure, as long as the set-point for spectroscopy (defining the transmission coefficient) and tip properties do not change within the spectral series. An even better reproduction of the sample LDOS is usually obtained when the $\mathrm{dI} / \mathrm{dV}$ signal is weighted by the junction resistance $(V / I)$ to account for bias-dependent changes of the transmission probability: ${ }^{97,98}$

Eq. $5 \quad \frac{d I}{d V_{0}} / \frac{V_{0}}{I} \propto \rho_{S}\left(r_{\text {Tip }}, e V_{0}\right)$

The dI/dV signal does not give a one-to-one map of the state density, as the underlying model contains a number of simplifications and assumptions that are not always met experimentally. ${ }^{60,70}$ However, Eq.4 gives a phenomenological description on how differential conductance spectra can be related to the electronic structure of the sample surface. For comparison with data obtained by conventional electron and photon spectroscopy (e.g. UPS and X-ray absorption spectroscopy), the spatial localization of a given electronic states has to be taken into account. Conductance spectroscopy with the STM captures only those states that have a large density probability in the vacuum region above the surface and overlap sufficiently strong with the tip wave functions. Consequently, mostly $s$ and $p$-like states show up in conductance spectra, while the more localized $d$ - and $f$-orbitals are hardly detected. ${ }^{99}$ In 
addition, occupied sample states are generally more difficult to observe than empty ones, as they are spatially more contracted and often located in the near-surface region in accordance to their binding character. Due to the spatial confinement and broken translational periodicity across an STM junction, the electron wave vector is not conserved during tunneling. ${ }^{60}$ Apart from a few model systems where the wave vector can be determined indirectly, ${ }^{100} \mathrm{dI} / \mathrm{dV}$ spectroscopy provides therefore no $k$-space information. The electron spin, on the other hand, is a conserved quantity and magnetic (spin) properties of the sample are detectable with STM. $^{101,102}$
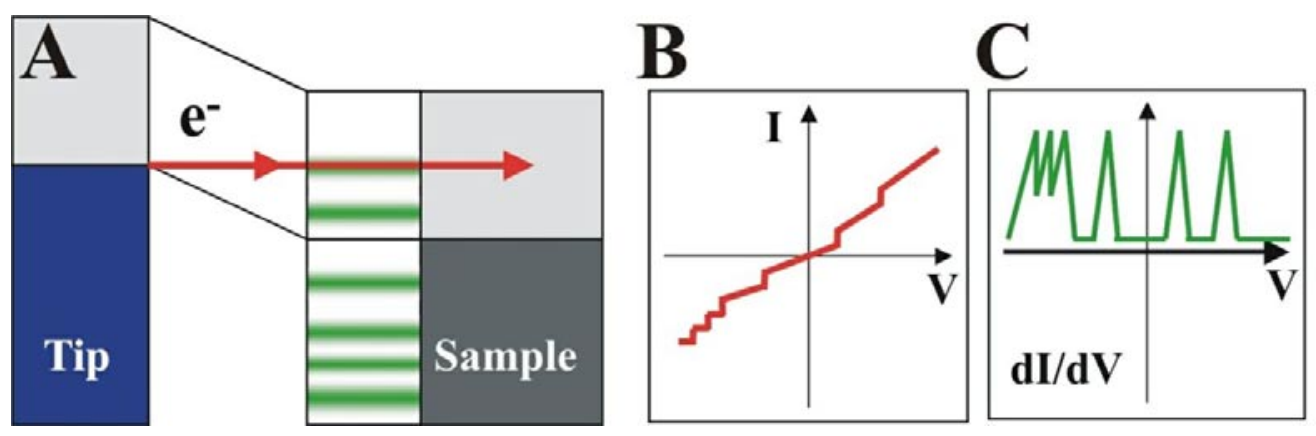

Fig. 2.7: (A) Potential diagram of an STM junction containing a system with discrete electronic states. (B) Current-voltage and (C) differential conductance curves modeled for the junction sketched in (A). Each energy level gives rise to a peak in the $\mathrm{dI} / \mathrm{dV}$ spectrum.

The $\mathrm{dI} / \mathrm{dV}$ signal is usually detected with lock-in technique, whereby a small modulation is added to the sample bias and the resulting current response is picked up by a frequency and phase-sensitive lock-in amplifier. Besides spectroscopy, also conductance imaging can be performed by recording the $\mathrm{dI} / \mathrm{dV}$ signal at a fixed bias on each point of a regular topography scan. This method provides information on the spatial distribution of electronic states and is employed for instance to map the shape and symmetry of molecular orbitals and quantum well states in a confined electronic system (see Ref.103,104 for examples).

The energy resolution of conductance spectroscopy is given by the Gaussian sum of the temperature and modulation-induced spectral broadening. ${ }^{105}$ For a certain intrinsic width of a LDOS peak $\Delta E_{i}$, the measured line width calculates to:

Eq. $6 \quad \Delta(e V)=\sqrt{\left(\Delta E_{i}\right)^{2}+\left(\Delta E_{T}\right)^{2}+\left(\Delta E_{M o d}\right)^{2}}$

Hereby, $\Delta E_{T}=3 k_{B} T$ denotes the temperature influence and $\Delta E_{M o d}=2.5 e V_{M o d}$ is the effect of the modulation bias. Whereas thermal broadening is the limiting factor for the energy resolution in room temperature studies $\left(\Delta \mathrm{E}_{\mathrm{T}}=77 \mathrm{meV}\right)$, the modulation bias contributes most 
to the measured line width in experiments performed at liquid helium temperature (e.g. 25 $\mathrm{meV}$ for $10 \mathrm{mV}$ modulation bias). For comparison, intrinsic line widths of different electronic modes range between $8 \mathrm{meV}$ for the $\mathrm{Ag}(111)$ surface-state onset ${ }^{106}$ and $300 \mathrm{meV}$ for the $6 s p$ resonance in Au adatoms on $\mathrm{NiAl}(110) .{ }^{107}$ Vibrational excitations, on the other hand, have a zero-temperature, zero-modulation width of only $4 \mathrm{meV}$, making an operation of the STM at cryogenic temperature absolutely necessary to detect the associated spectral features. ${ }^{105}$ In the context of this work, conductance spectroscopy is mainly applied to investigate the gap size of oxide materials, the spectral signature of localized defects and impurities as well as the electronic properties of single adsorbates and metal aggregates on oxide supports.

\subsection{Inelastic tunneling spectroscopy}

Electron tunneling between tip and sample does not only take place in the elastic regime, but might involve the transfer of energy to localized excitations in the tip-sample gap. Energy losses of tunneling electrons can stimulate vibrational and translational modes of individual molecules, ${ }^{108,109}$ as well as optical ${ }^{110}$ and magnetic excitations in the tunnel junction. ${ }^{111,112}$ The spectroscopic approach to probe inelastic transport channels is termed inelastic-electrontunneling spectroscopy (IETS) with the STM. As IETS is not in the focus of this review, the technique will be discussed only briefly here, while a detailed introduction can be found in the literature. $^{109,113}$
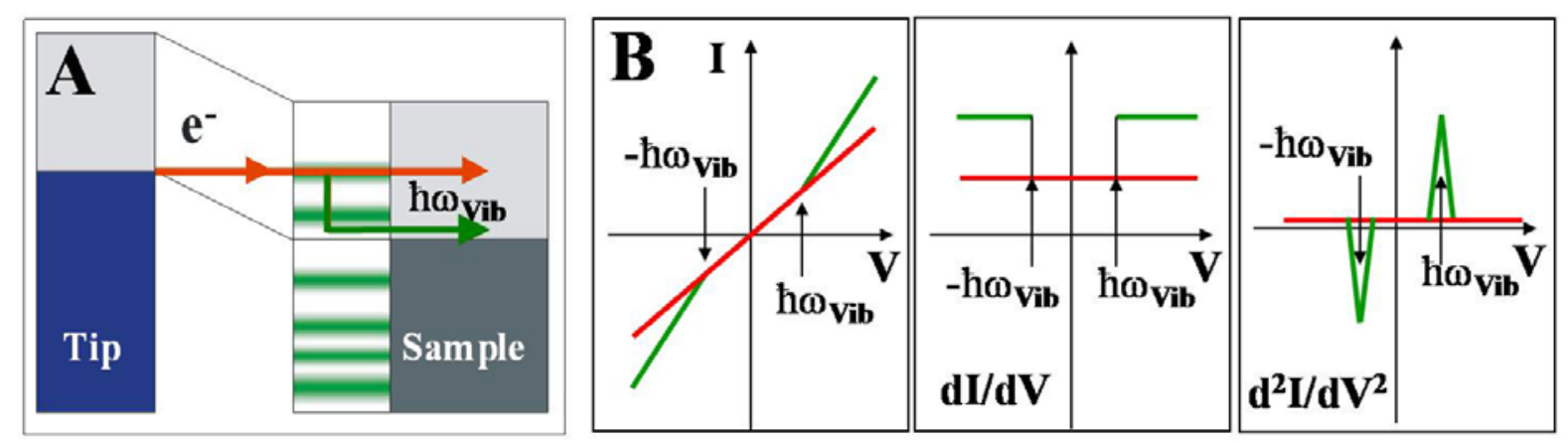

Fig. 2.8: (A) Visualization of the elastic (red) and inelastic (green) transport channel in an STM junction. The resulting current-voltage characteristic and its first and second derivatives are depicted by red (only elastic channel) and green lines (elastic and inelastic channel) in (B).

The opening of an inelastic transport channel by exciting a mode in the tip-sample gap usually leads to an increase of the total conductance, as elastic and inelastic transport contributions add up (Fig. 2.8). In selected cases, interference effects between elastic and inelastic channels may also result in a decreasing $\mathrm{dI} / \mathrm{dV}$ signal. ${ }^{114}$ In both cases, a small conductance step occurs 
at the bias position, at which the energy of the tunneling electrons matches the excitation energy of the gap mode. As changes in the differential conductance are small (1\%-10\%), the second bias-derivative of the tunnel current $\left(\mathrm{d}^{2} \mathrm{I} / \mathrm{dV}^{2}\right)$ is usually monitored instead of the $\mathrm{dI} / \mathrm{dV}$ signal. ${ }^{109}$ In the resulting $\mathrm{d}^{2} \mathrm{I} / \mathrm{dV}^{2}$ spectra, the bias onset for inelastic tunneling shows up as a symmetric peak/dip structure at positive/negative bias (Fig. 2.8). This spectral symmetry reflects the similar excitation cross-section of inelastic modes via electron tunneling into and out of the sample and provides a first hint for the inelastic nature of the spectral feature. A reliable identification demands, however, for independent proof, for instance by verifying a vibrational origin with isotope exchange experiments.
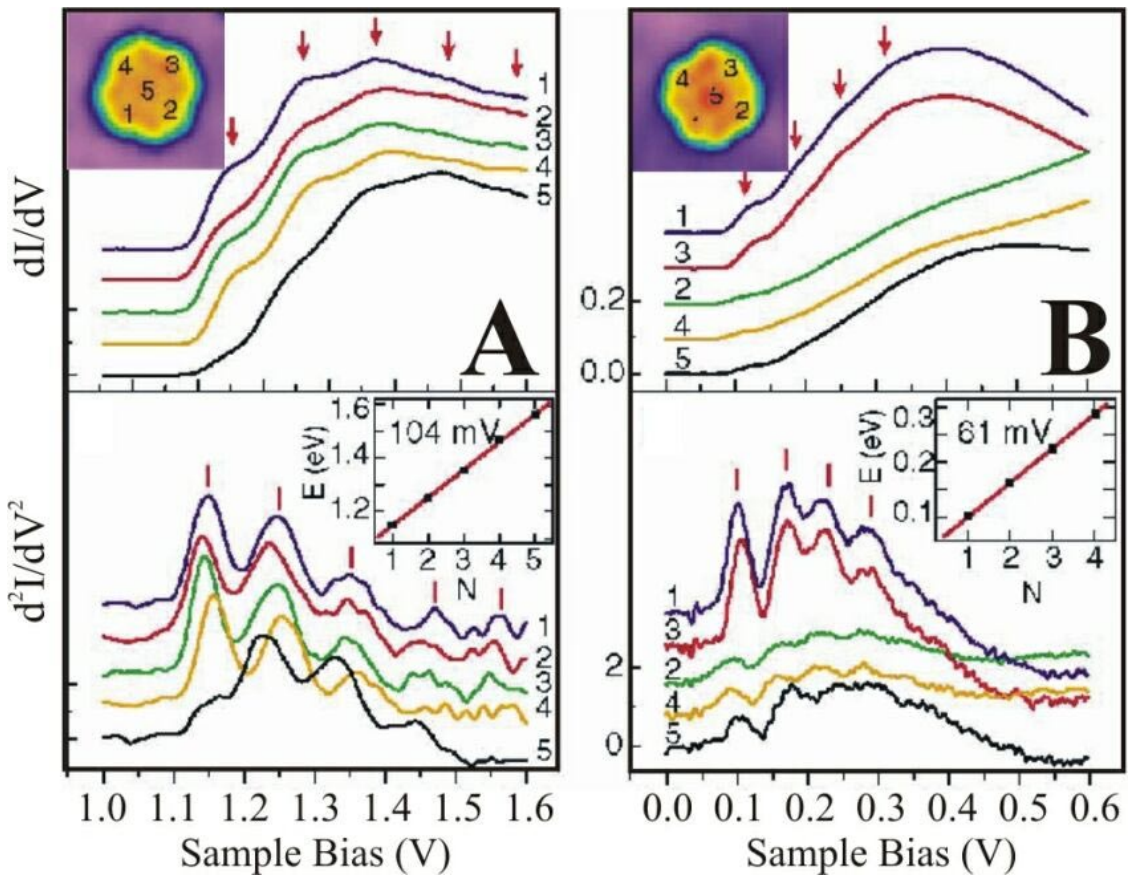

Fig. 2.9: (A,B) $d I / d V$ (top) and $d^{2} I / d V^{2}$ spectra (bottom) of CuPc molecules on alumina/NiAl(110) in two configurations. While the $\mathrm{dI} / \mathrm{dV}$ spectra show a series of equidistant kinks (marked by arrows), pronounced peaks become visible in the second-derivative spectra. The features result from tunneling through a vibrational progression. The peak-separation, as displayed in the insets, corresponds to the vibrational energy. At variance to classical IETS, the peaks are not centered at the Fermi level, but start at the LUMO of the molecule. ${ }^{115}$

The technique of IETS with the STM has not yet become very important for characterizing oxide materials, although the list of potential experiments is long. IETS could in principle be applied to detect phonon, magnon and exciton modes in bulk and thin-film oxides as well as to study vibrational properties of oxide-supported molecules. Only in the latter case, considerable progress has been reported in recent years, as reflected in various IETSexperiments on single phthalocyanine ${ }^{115,116}$ and fullerene molecules ${ }^{117}$ on alumina thin films (Fig. 2.9). Several vibrational modes could be indentified in these studies and correlated with 
the binding configuration of the molecules on the oxide surface. In contrast to conventional IETS, the vibrational signature appears as a progression of peaks centered on an eigenstate of the molecule (here the LUMO). The presence of the insulating spacer turned out to be essential for the detection of the vibrational progression, because it leads to an enhanced lifetime of the electronically excited molecule and enables significant coupling to its vibrational degrees of freedom. Not surprisingly, no vibrational fine-structure was observed in the $d^{2} I / d V^{2}$ spectra when the same molecule had been deposited onto a metal support.

\subsection{Photon emission spectroscopy with the STM}

Inelastic electron transport can also induce optical activity in an STM junction. ${ }^{110}$ In contrast to IETS that utilizes the electron current as a source of information, photon emission spectroscopy with the STM directly detects the light from the tip-sample gap. This technique offers the unique possibility to measure optical properties of surfaces with a spatial resolution of a few nanometers, a value that only depends on the diameter of the exciting electron beam from the tip. Light emission spectroscopy with the STM is well suited to probe the optical characteristics of oxide surfaces at the local scale. The main mechanisms of photon emission from an STM junction shall therefore be sketched in the following, while more details can be found in the literature. ${ }^{110}$

Two main principles govern the light emission from an STM junction:

(i) Excitation of coupled electromagnetic modes in the tip-sample gap (so called tip-induced plasmons) via inelastic electron tunneling.

(ii) Radiative recombination of the hot carriers (electrons, holes) injected from the tip into the sample surface (electro-luminescence).

While the first mechanism is typical for metal samples, as their free-electron character supports plasmonic excitations, ${ }^{118,119}$ the second one occurs preferentially in materials with a gap in their state-density, e.g. in semiconductors, ${ }^{120,121,122}$ oxides, ${ }^{51}$ quantum wells ${ }^{123}$ and single molecules. ${ }^{124,125}$ Common to both scenarios is the crucial role of the electromagnetic near-field in the tip-sample cavity, which amplifies the emission cross-section. ${ }^{126,127}$ Thanks to this field enhancement, light emission from an STM junction becomes an observable effect, in spite of the extremely local character of the measurement $\left(\sim \mathrm{nm}^{2}\right)$, the small electron flux from the tip ( nA) and the finite number of optical modes involved.

The first comprehensive treatment of the field enhancement in the tip-sample gap of an STM has been given by Johansson et al. ${ }^{126}$ He simulated the tunnel junction with a metal sphere 
placed in front of flat surface and solved the Laplace equation for this model geometry by considering the appropriate boundary conditions (Fig. 2.10). The field enhancement of the STM junction was then obtained by dividing the induced electromagnetic near-field $E_{\text {ind }}$ below the tip by a virtual far-field of unity strength $E_{\text {inc }}$. The resulting field amplification was found to depend weakly on the junction geometry, as given by tip-radius $R$ and tip-sample distance $z$, but strongly on the dielectric properties of tip and sample $\left(\varepsilon_{1}, \varepsilon_{2}\right)$. The enhancement factor can reach values of several thousand for noble metal junctions exhibiting small dielectric losses, and of 10-100 for transition-metal and semiconductor samples. In the frequency domain, the field enhancement peaks slightly below the plasmon frequency of an imaginary sphere made from the more noble of the two electrode materials, while directly at the plasmon resonance the enhancement drops to zero (Fig. 2.10). ${ }^{126,127}$ At this particular frequency, the electromagnetic gap mode decomposes into localized excitations in tip and sample. The physical origin for the strong near-field in the tip-sample cavity lies in the excitation of coherent and coupled oscillations of the electron gases in both electrodes. ${ }^{118}$ These concerted modes have been termed 'tip-induced plasmons' (TIPs), following the common nomenclature for collective electronic excitations in solid-state physics. The electromagnetic near-field in the tip-sample junction is spatially confined to an area with 40$200 \AA$ diameter, depending on the tip radius and the tip-sample distance. ${ }^{128}$ This strong fieldlocalization in conjunction with large amplification factors is the basic requirement to perform optical spectroscopy with an STM at the nanometer scale.
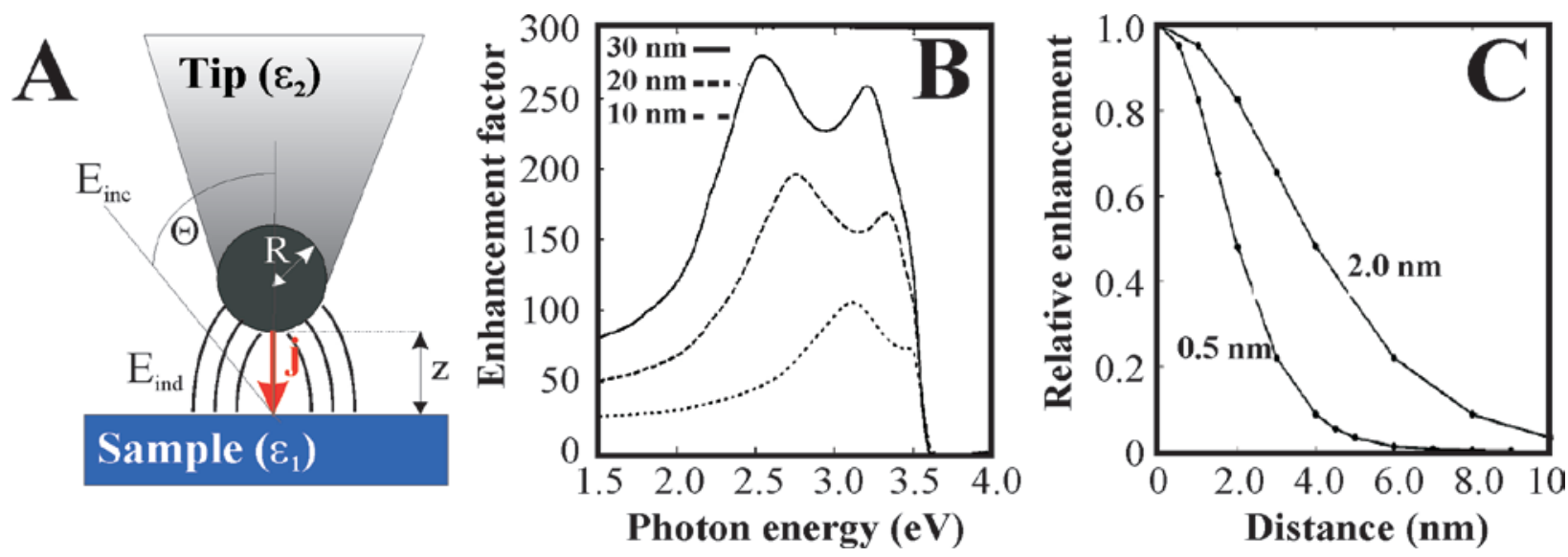

Fig. 2.10: (A) Model geometry used for calculating the field enhancement in the tip-sample gap. The enhancement factor is defined as the ratio between the induced near-field below the tip $E_{\text {ind }}$ and a driving far-field $E_{\text {inc }}$. (B) Calculated enhancement factors for iridium spheres with three different radii $R$ above a silver surface. The two electrodes are separated by $0.5 \mathrm{~nm}$. The enhancement vanishes at 3.5 $\mathrm{eV}$, when the gap mode localizes within the two electrodes. From [126]. (C) Dependence of the enhancement factor on the lateral distance from the tip-sample axis for two different vertical separations $\mathrm{z}$, as calculated for an Ag-Ag contact. ${ }^{128}$ 
The tip-induced plasmons need to be stimulated by inelastically tunneling electrons in the STM junction, representing the physical source of energy. In the Johansson model, ${ }^{126}$ the inelastic tunnel current $j$ is calculated from summing up all possible transitions between initial $|i\rangle$ and final states $|f\rangle$ in tip and sample, whose eigenenergies $\varepsilon_{i}$ and $\varepsilon_{f}$ are separated by the plasmon energy $\hbar \omega$ to obey energy conservation: $\varepsilon_{i}-\varepsilon_{f}=\hbar \omega$. For sake of simplicity, the underlying wave functions are assumed to be free-electron like and their decay into the gap region is deduced from solving the one-dimensional Schrödinger equation for a trapezoidal barrier. The radiated electromagnetic field $E(r, \omega)$ is finally determined from the reciprocity theorem of electrodynamics, using the inelastic tunnel current $j$ and the calculated field enhancement $G\left(\Theta, r^{\prime}, \omega\right)$ in the gap: ${ }^{126}$

Eq. 7. $\quad E(r, \omega)=\int d^{3} r^{\prime} \frac{\omega}{c^{2}} \frac{e^{i k r}}{r} G\left(\Theta, r^{\prime}, \omega\right) \cdot j\left(r^{\prime}, \omega\right)$

In principle, photon emission from the STM junction can now result from the direct decay of tip-induced plasmons, as typically observed for metal samples (Fig. 2.10), ${ }^{110,118}$ or from radiative electron transitions in a sample with a direct band gap. For the latter mechanism, which is the relevant one in the context of oxide materials, the emission characteristic is governed by the optical properties of the sample, and the tip mainly provides the hot electrons/holes to stimulate the optical activity. However, also in this case, the fieldenhancement in the STM gap leads to an amplification of the photon yield with respect to the isolated sample. In the most basic model, the enhanced yield can be estimated from the initial electron-transition probability $T_{i \rightarrow f}(\hbar \omega)$ of the isolated emitter, as given by Fermi's Golden Rule, multiplied with the field-enhancement factor $G(\hbar \omega)$ of the STM cavity. ${ }^{128}$ Thanks to the enhancement effect of the STM junction, even weak photon sources, such as single molecules, might produce sufficiently high photon fluxes to be detected in the far field, which has been exploited for instance to realize tip-enhanced Raman spectroscopy in an STM setup. ${ }^{129}$

Photon emission spectroscopy with the STM can be employed to study the local optical properties of pristine as well as adsorbed-covered oxide surfaces. Light emission from widegap materials usually stems from the radiative recombination of electron-hole pairs (excitons), following an electronic or optical excitation (electro- or photoluminescence). ${ }^{130}$ The trapping and subsequent annihilation of excitons occurs preferentially at low-coordinated lattice sites 
or defects, where the Madelung potential is smaller and the electron-hole pairs can reduce their total energy. ${ }^{131,132,133}$ Spatially-resolved optical experiments with an STM are therefore a powerful means to probe the defect structure of oxide surfaces. This is especially true, because structural parameters of the emission centre and its specific optical behavior can be explored simultaneously at the local scale. The feasibility of this approach has been demonstrated only for $\mathrm{MgO}$ thin films on $\mathrm{Mo}(001)$ so far, where two exciton-mediated emission bands could be identified in the optical spectra upon electron injection from the tip (Fig. 2.11). ${ }^{51,82}$ However, electron energies of more than $50 \mathrm{eV}$ were required to stimulate the photon response, which limited the spatial resolution of the experiment to some hundred nanometers and rendered the identification of individual optical centers impossible. An increase in spatial resolution can however be achieved, when a narrow-gap oxide is used for optical studies that requires lower excitation energies for the excitons.
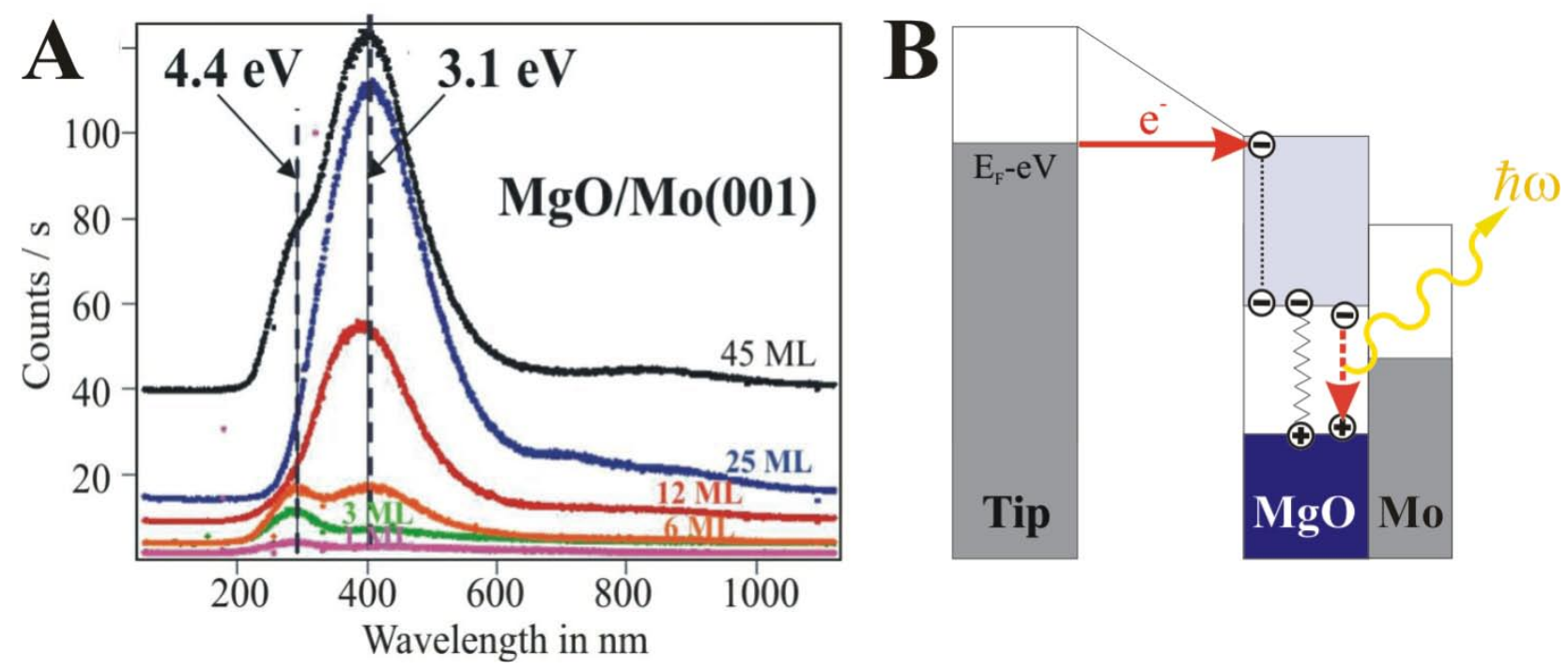

Fig. 2.11: (A) Optical emission spectra of $\mathrm{MgO} / \mathrm{Mo}(001)$ films with given thickness, excited by electron injection from the STM tip into the surface $\left(E_{\text {tip }}=-150\right.$ V). (B) Proposed emission mechanism: MgO excitons are created first by an energy transfer from the incoming electrons. The electron-hole pairs then diffuse across the surface until they become trapped and decay at low coordinated lattice sites. The two optical bands observed here are assigned to exciton recombination at step edges $(4.4 \mathrm{eV})$ and corner sites $(3.1 \mathrm{eV})$ in the MgO surface. ${ }^{51}$

In contrast to bare oxide surfaces, photon emission spectroscopy with the STM has been widely employed to investigate the optical response of single metal particles ${ }^{119,134}$ and molecules on oxide supports. ${ }^{124,125}$ In the case of metal particles, the emission mechanism is governed by the excitation of collective electronic modes in the particle electron gas (so called Mie plasmons) followed by their radiative decay. ${ }^{58}$ An example for the resulting optical response is given in Fig. 2.12 for single $\mathrm{Au}$ particles on a $\mathrm{TiO}_{2}$ bulk support. ${ }^{135,136}$ The 
injection of tip electrons with $5 \mathrm{eV}$ energy into the particle centre produces a characteristic emission peak at $550 \mathrm{~nm}$ that can be assigned to a Mie-plasmon excitation in the hemispherical Au particle. The emission intensity starts to decline when the tip is moved towards the particle edge and completely vanishes for electron injection into the oxide support. From this spectral series taken across the particle, the spatial resolution of the method can be estimated to be around $1 \mathrm{~nm}$, which is well below the resolution limit of conventional optical techniques set by the wavelength of the emitted radiation. Further experiments have been performed for Ag and Ag-Au alloy particles on alumina thin films, putting special emphasis on the interrelation between the plasmon-mediated emission behavior and the geometry and chemical composition of the individual particles. ${ }^{119,137}$ For Ag clusters, for example, a blue-shift of the plasmon resonance was revealed with decreasing particle diameter, ${ }^{119}$ reflecting the diminishing influence of the Ag $d$-electrons on the plasmon energy in aggregates with large surface-to-volume ratio. ${ }^{138}$
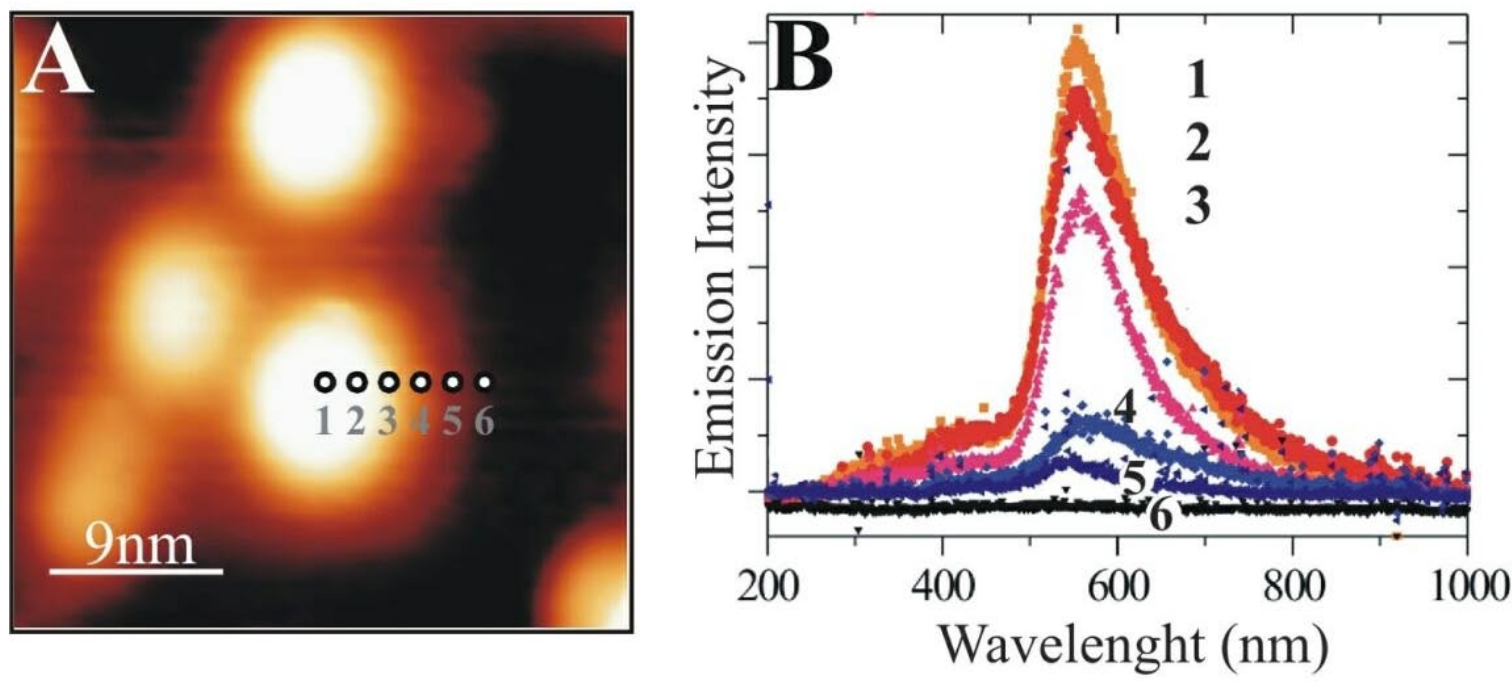

Fig. 2.12: (A) STM image of Au particles on $\mathrm{TiO}_{2}(110)$. (B) Corresponding photon emission spectra taken across the central particle at the positions marked in (A) $\left(\mathrm{U}_{\mathrm{s}}=5 \mathrm{~V}, \mathrm{I}=2 \mathrm{nA}\right)$. The plasmonmediated light emission is only observed when the tip electrons are injected into the particle.

STM-induced light emission has also been observed for individual Zn-Etiol molecules deposited on alumina/NiAl(110), as shown in $.2 .13 .^{124}$ The emission is initiated by electron injection into an unoccupied molecular orbital located at 2.1-2.5 eV above the Fermi level. From there, the electrons decay into a low-lying molecular state and emit a photon with 1.5$1.7 \mathrm{eV}$ energy. The emission process might therefore be regarded as a LUMO+1 to LUMO transition within the molecule. The most intriguing experimental result is, however, the reproducible observation of a fine structure in the molecular light emission, consisting of a series of sharp sub-peaks with constant energy separation of $(40 \pm 2) \mathrm{meV}$. This energy value is 
in good correspondence with a vibrational excitation of the molecule, suggesting that the peak series is in fact a vibrational progression that accompanies the electronic transition. The strong electron-phonon coupling responsible for this effect is partly induced by the oxide spacer layer, which efficiently decouples the molecular electronic system from the metal support and increases the lifetime of the excited molecule.
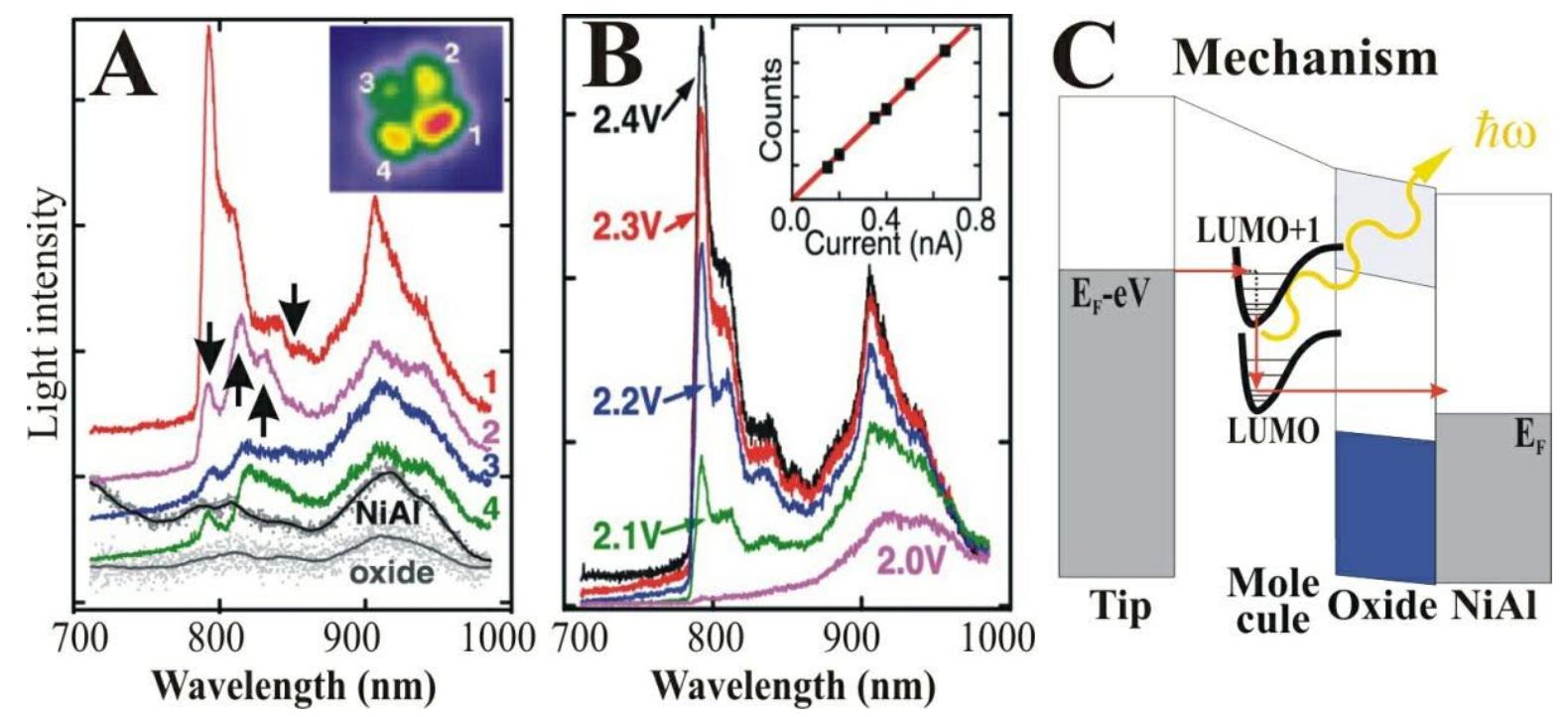

Fig. 2.13: (A) Luminescence spectra of different lobes of a single ZnEtiol molecule (shown in the inset) deposited on a thin alumina film on $\mathrm{NiAl}(110)$ (Excitation bias $\mathrm{V}_{\text {Sample }}=2.35 \mathrm{~V}$ ). Arrows mark the sub-peaks that are related to a vibrational progression accompanying the optical transition. (B) Similar spectral series, but shown as a function of the excitation bias. The current dependence of the emission intensity is depicted in the inset. (C) Proposed mechanism for the photon emission from the molecule in the tip-sample junction. ${ }^{124}$

Despite of the large potential of photon emission spectroscopy with the STM, the method has been applied only in a few cases to investigate the local optical properties of oxides as well as molecules and clusters adsorbed on their surface. The little spread of the technique is mainly owed to the experimental challenge to detect the tiny photon fluxes resulting from electron injection into a nanometer-sized surface region. Additionally, single adsorbates do often not withstand the excitation energies that are required to activate an optical mode, and desorb upon electron injection. However, as STM-mediated photon emission spectroscopy is one of the few methods that enable simultaneous exploration of the topography and optical properties of a surface, it will certainly gain more attention in future studies on oxide systems. Possible objectives of those experiments will be the investigation of oxide excitons, in particular their diffusion, trapping and recombination behavior on well-characterized surfaces. Also single metal particles as well as molecules bound to oxide surfaces will remain in the focus of the 
experiments. Hereby, possible energy-exchange mechanisms between particle plasmons and single-electron excitations in molecules and the oxide support need to be explored, as they form the basis for processes occurring in photo-catalytically active or dye-sensitized oxide systems. $^{15,139}$

As demonstrated in the previous paragraphs, the STM is a powerful tool to investigate the structural, electronic, vibrational and optical properties of oxide materials at the nanometer scale. A large number of examples that will demonstrate this potential will be given in the following chapters. However, despite of the possibility for atomic-scale studies, the STM develops its full potential only in combination with other, spatially-averaging techniques. In particular, an independent chemical analysis is often essential to assign adsorbates occurring in the STM images to the correct chemical entities. ${ }^{44}$ Also structural information obtained by STM, e.g. on the diameter of metal particles or the height of adsorbates above the surface, should be verified by independent probes, such as diffraction techniques or electron microscopy. This is required, because the STM tip introduces a certain degree of uncertainty into the measurements due to its ill-defined shape and chemical identity. Closely related to this, spectroscopic measurements with the STM are always subject to tip influences and need to be compared with the results of averaging spectroscopic techniques and theoretical studies.

As a conclusion, the STM is able to contribute to a better understanding of oxide surfaces in various ways; however a comprehensive and reliable picture of oxide materials only emerges upon combination with other surface science techniques. 


\section{Oxide thin films and their surface properties}

The preparation of oxides in the form of thin films opens the unique possibility to study this class of materials with the well-established methodology of surface science, as it overcomes the restrictions imposed by the insulating nature of many bulk oxides. ${ }^{27,46,47,48,49,53}$ However, it brings about a number of systematic differences in the structural, electronic and chemical properties of the low-dimensional system with respect to the bulk material. Furthermore, a number of technical questions need to be solved, e.g. finding a suitable support material and the optimal thermodynamic conditions for film preparation. The first step towards a defectpoor oxide layer is the formation of a structurally well-defined and crystalline interface with the metal support. With increasing thickness, this precursor layer should transform into a stable bulk phase, which usually involves the relaxation and reconstruction of the interfacial oxide planes. Ideally, a film thickness can be prepared at which the characteristic bulk properties emerge in the top oxide-layers, but unwanted charging effects are still suppressed. In this case, a thin oxide film on a metal substrate represents a suited model system for the respective bulk material what its surface properties and adsorption behavior concerns. Following this outline, the first section of this chapter discusses the nucleation and growth of thin oxide films on metal supports, emphasizing in particular the role of the STM to analyze the underlying processes at the atomic scale.

\subsection{The metal-oxide interface: Structural aspects}

The structural properties of the metal-oxide interface decisively govern the crystalline nature and defect concentration of the oxide film. A suitable metal support for film growth is characterized by a number of parameters:

(i) The atomic arrangement in the metal surface should match the symmetry of the oxide unit cell along the desired growth direction (Fig. 3.1). On metal surfaces with a square lattice, the nucleation of square oxide phases is favored [e.g. $\mathrm{CoO}(001),{ }^{79} \mathrm{NiO}(001)^{140,141}$ and $\mathrm{MgO}(001)^{142,143,144}$ on $\mathrm{Ag}(001)$ and $\left.\mathrm{MgO}(001) / \mathrm{Mo}(001)^{51,145}\right]$, whereas a hexagonal metal surface induces the growth of oxide films with six-fold symmetry [e.g. $\mathrm{FeO}(111) / \mathrm{Pt}(111)^{146,147}$ and $\left.\mathrm{MgO}(111) / \operatorname{Ag}(111)^{148,149}\right]$. As a consequence, the growth direction of the oxide layer can be controlled to a certain extent by choosing the correct substrate symmetry. Exploiting this 
template effect, oxide films can be stabilized even when their structure does not correspond to the thermodynamically favored phase, which is the decisive precondition to prepare polar oxide films. However, also deviating symmetries for oxide and metal substrate have been reported in the literature, especially for chemically inert metal supports. In these cases, the metal-oxide interaction is not sufficient to determine the oxide geometry and the oxide grows along its favored crystallographic direction. For example, $\mathrm{NiO}^{150}$ and $\mathrm{MnO}^{151}$ were found to expose mainly (001) planes even on the hexagonal Pt(111) surface.

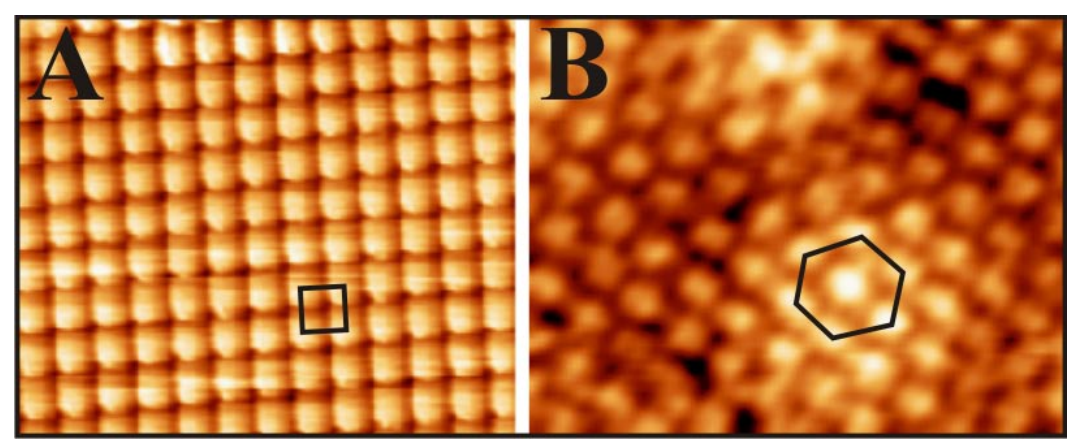

Fig. 3.1: STM images of two oxide films with rocksalt structure, exposing different crystallographic planes: (A) $\mathrm{MgO} / \mathrm{Ag}(001)$ and (B) $\mathrm{FeO} / \mathrm{Pt}(111)\left(3.5 \times 2.9 \mathrm{~nm}^{2}\right)$. The different surface symmetries reflect the influence of the support on the growth direction. The topographic height of the FeO film is modulated, as Fe atoms occupy different binding sites on the Pt surface.

(ii) Apart from the symmetry match, the formation of high-quality interfaces requires a small difference between metal and oxide lattice constants, whereby the lattice mismatch must not exceed $5 \%$ to enable pseudomorphic growth. ${ }^{142}$ For a larger mismatch, the oxide film reconstructs or forms coincidence structures already in the first layer, ${ }^{146}$ which has usually consequences for the quality of thicker films. For example, while $\mathrm{MgO}$ films grown on $\mathrm{Ag}(001)$ are smooth and defect free due to a lattice mismatch of $3 \%$ only, ${ }^{143}$ the $\mathrm{MgO} / \mathrm{Mo}(001)^{152}$ system with $5.4 \%$ mismatch develops a defect network already in the interfacial layer. It should be noted that due to an intrinsically high lattice mismatch, natural oxide films prepared by oxidation of single-crystalline metal surfaces have often low structural quality. ${ }^{153,154}$ This problem can be circumvented, when binary alloys are used as starting point, whereby only one component becomes oxidized (e.g. alumina on $\mathrm{NiAl}$ and $\mathrm{CuAl}) .{ }^{155,156}$ On the other hand, the growth of oxide layers on bulk oxides usually results in films of extremely high quality and low interface-roughness, reflecting the similar lattice constants of many rocksalt, corundum and spinel structures being fixed by their oxygen sublattice. ${ }^{157,158}$

(iii) Support and ad-layer should be non-miscible at the interface and the support should have a good thermal and mechanical stability. The latter conditions enable high-temperature 
treatment of the as-grown film, facilitating a transition into the thermodynamic equilibrium. ${ }^{152}$ Oxide films on high-melting substrates (e.g. NiAl, Pt, Mo and W) therefore often exhibit a superior crystallographic quality and a lower defect concentration compared to films on lowmelting supports, as Ag or $\mathrm{Cu}$. The possibility to anneal the metal-oxide system to high temperatures is especially advantageous when thick oxide films shall be grown. ${ }^{152}$

\subsection{The metal-oxide interface: Electronic aspects}

The adhesion between the metal surface and the oxide over-layer is often of pivotal importance for the stability and crystallographic properties of the film. ${ }^{50}$ For non-reactive supports, the interfacial binding strength is usually weak, imposing fewer constraints on a perfect lattice match and good interfacial registry. ${ }^{28,151}$ Typical systems falling into class are $\mathrm{MgO}^{142,143}$ and $\mathrm{NiO}^{142,150}$ films on $\mathrm{Ag}(001)$. For metal supports with higher reactivity, on the other hand, the interface contribution might even exceed the intrinsic lattice energy of the oxide, and the film structure becomes entirely governed by the symmetry and lattice parameters of the substrate. ${ }^{29,159}$ Examples for this behavior are the thin alumina films grown on the various $\mathrm{NiAl}$ surfaces, as discussed in the second part of this paragraph. ${ }^{48,160,161}$

For weak or moderate oxide-support interactions, the interfacial coupling strength mainly determines the registry of atoms in the oxide and support, and to a smaller extent the oxide lattice constant. In most cases, the metal-oxide adhesion strength can be directly correlated with the oxygen affinity of the substrate. ${ }^{162}$ Metals with high oxidation enthalpies have a strong tendency to form interfacial bonds with the oxygen ions in the film. The $\mathrm{O}$ atoms therefore mainly occupy the top positions in the metal surface. For $\mathrm{MgO} / \mathrm{Mo}(001)$ and $\mathrm{MgO} / \mathrm{Pd}(001)$ systems, for example, the formation of O-Mo and O-Pd bonds is strongly preferred over interfacial metal-metal bonds. ${ }^{162,163}$ The strength of an interface bond is also reflected by the average metal-oxide separation. While the MgO-Ag(001) interlayer distance has been calculated to $2.73 \AA$, this value decreases to $2.15 \AA$ for $\mathrm{MgO}$ on a Mo(001) support. ${ }^{164}$ The true nature of the interfacial metal-oxygen bonds needs to be analyzed via density-function-theory (DFT) calculations, as carried out for various model systems such as $\mathrm{Pd} / \mathrm{MgO},{ }^{162} \mathrm{MgO} / \mathrm{Ag}(001){ }^{165}$ and $\mathrm{FeO} / \mathrm{Pt}(111) .{ }^{166}$ For the $\mathrm{Pd} / \mathrm{MgO}$ case, as an example, the interaction originates from a hybridization between the $O-2 p_{x y}$ orbitals in the oxide film and the Pd $d_{x z, y z}$-states in the support. ${ }^{162}$ Formation of those Pd-O bonds represent the largest contribution to the interfacial adhesion between metal and oxide film. 
On metal-oxide systems with pronounced lattice mismatch, where the interfacial registry shifts from cations to anions being bound atop a substrate atom, the layer exhibits pronounced height modulations due to the different interface-bond lengths. These height variations have indeed been indeed observed with STM for the $\mathrm{MgO} / \mathrm{Mo}(001)$ system. In topographic images of mono-layer thick films, lattice regions with $\mathrm{Mg}$ atoms bound on-top of the Mo sites show up as a network of bright lines due to the enlarged Mg-Mo binding length (Fig. 3.2A). ${ }^{152}$ In selected cases, the oxygen affinity of the support material is so strong that the number of cations in the oxide-interface layer is reduced to allocate sufficient space for the anions to interact with the metal surface. For a $\mathrm{NiO}$ monolayer on $\mathrm{Pd}(001)$, an ordered network of $\mathrm{Ni}$ vacancies has been inferred from STM and LEED measurements, which enables the formation of particularly strong Pd-O bonds at the interface and reduces the lattice strain in the oxide film. ${ }^{167}$

The registry at the metal-oxide interface can be deduced from STM images taken in the early growth regime of the oxide film when both, the substrate and oxide atoms, are still resolved within one image. ${ }^{78}$ Similar information might be obtained from the reverse approach that means from depositing metal islands onto crystalline oxide surfaces (Fig. 3.2B). ${ }^{168}$ For compact, thick oxide films, on the other hand, diffraction techniques and electron spectroscopy may provide insight into the nature of the metal-oxide interface. ${ }^{163,169}$
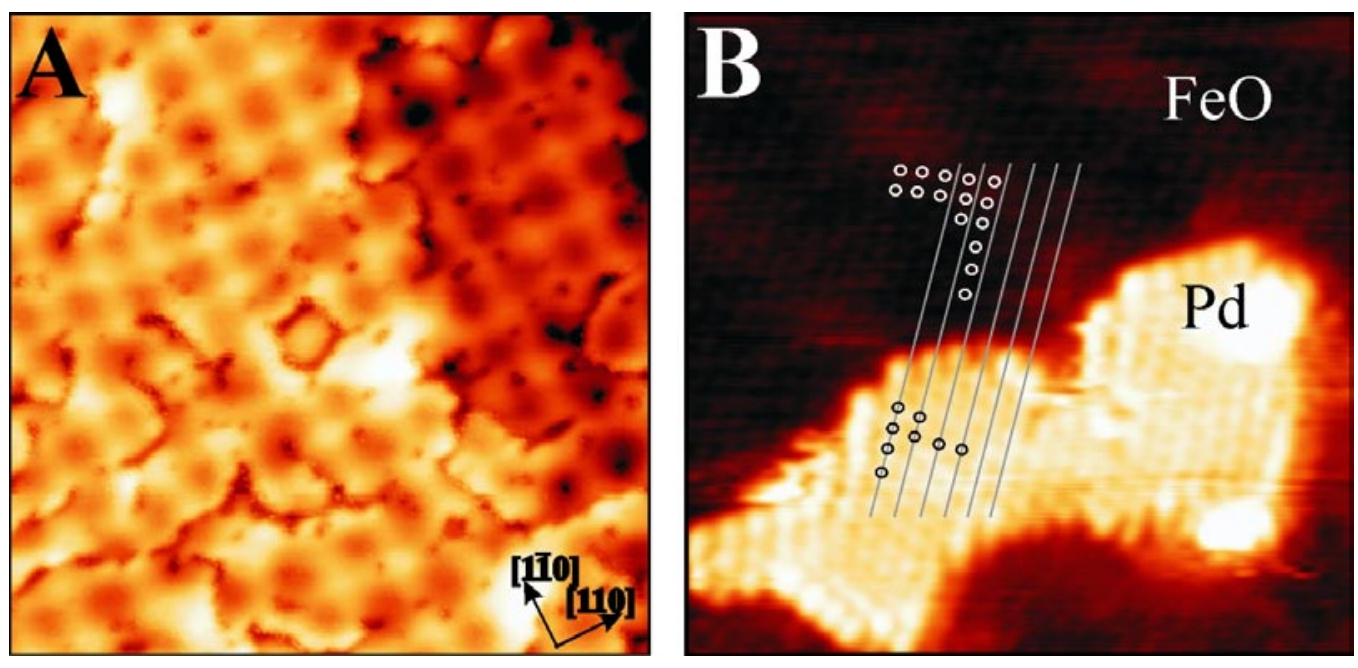

Fig. 3.2:

(A) STM image of a $2 \mathrm{ML}$ MgO film on $\mathrm{Mo}(001)\left(50 \times 50 \mathrm{~nm}^{2}\right)$. The square pattern of bright lines marks the oxide areas where $\mathrm{Mg}$ atoms are located on top of the Mo atoms in the support. The dark, irregular lines are domain boundaries in the oxide film. ${ }^{51,152}$ (B) Atomically resolved STM image of a two-dimensional Pd island formed by room temperature deposition onto $\mathrm{FeO} / \mathrm{Pt}(111)\left(9.0 \times 9.0 \mathrm{~nm}^{2}\right)$. The atom positions in island and film are in an out-of-phase relation, indicating that the Pd occupies three-fold hollow sites of the atomic species imaged in the film. According to DFT calculations, ${ }^{166}$ the resolved species are the Fe ions, suggesting that Pd binds on top of $\mathrm{O}$ species in the FeO surface. 
The metal-oxide coupling across the interface also determines the stacking sequence of layered or polar oxide films. For metal substrates with high oxygen affinity, the oxide usually starts with an oxygen plane at the interface, as observed for $\mathrm{SiO}_{2}$ on $\mathrm{Mo}(112)^{170}$ and $\mathrm{RhO}_{2}$ on $\mathrm{Rh}(110) .{ }^{171}$ In contrast, the interface is formed by a cationic oxide layer for more inert metal supports, as revealed for FeO films on $\mathrm{Pt}(111)$ for example. ${ }^{28,172}$ Also the electro-negativity of the support material plays a crucial role for the nature of the interfacial layer. ${ }^{173}$ Strongly electronegative metals, such as $\mathrm{Pt}$ and $\mathrm{Au}$, attract charges from the oxide film, rendering the interface layer slightly electron deficient (Fig. 3.3). In this case, a cationic plane at the interface is highly preferred over an oxygen layer that is electronegative itself. For electropositive materials, the sequence is reversed and formation of an oxygen plane in contact with the metal is favorable from the electrostatic point of view (Fig. 3.3).
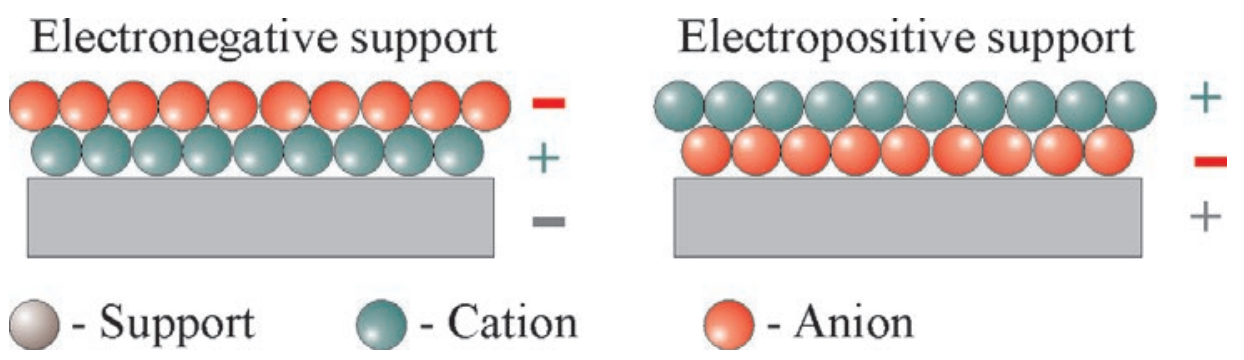

Fig. 3.3: Sequence of interfacial oxide layers for an electronegative (left) and an electropositive support (right). Only the electrostatics of the metal-oxide interface is considered in this case. The dominant charge state of the different layers is marked by positive and negative signs, respectively. ${ }^{173}$

In selected cases, the amount of hybridization at the metal-oxide interface can directly be deduced from the contrast obtained in STM topographic images. The tunneling probability is usually high, when electrons are injected into hybrid states formed between the metal and oxide atoms at the interface. This is nicely demonstrated for the FeO/Pt(111) system, which develops a coincidence structure with either $\mathrm{Fe}$ or $\mathrm{O}$ atoms being in on-top position of the Pt(111) lattice (Fig. 3.4). ${ }^{166,174}$ In low-bias STM images (150 mV), those oxide regions appear bright, where the $\mathrm{O}$ atoms occupy on-top sites and hybridization between the $O-2 p_{z}$ and the $\mathrm{Pt}$ states locally increases the available state density for electron transport (Fig. 3.4). In contrast, at an intermediate bias voltage $(500 \mathrm{mV})$, the $F e-d_{z} 2$ states dominate the LDOS of the oxide film while the O-derived state density is comparatively small. The tunneling probability into the film becomes now particularly high, when Fe and Pt atoms sit on top of each other and their atomic orbitals are able to interfere. Consequently, the bright topographic contrast moves from the O-top regions to adjacent areas with Fe sitting atop. The imaging contrast in the 
STM therefore provides a first indication for the amount of interfacial hybridization, which might facilitate the analysis of the genuine properties of the metal-oxide interface.
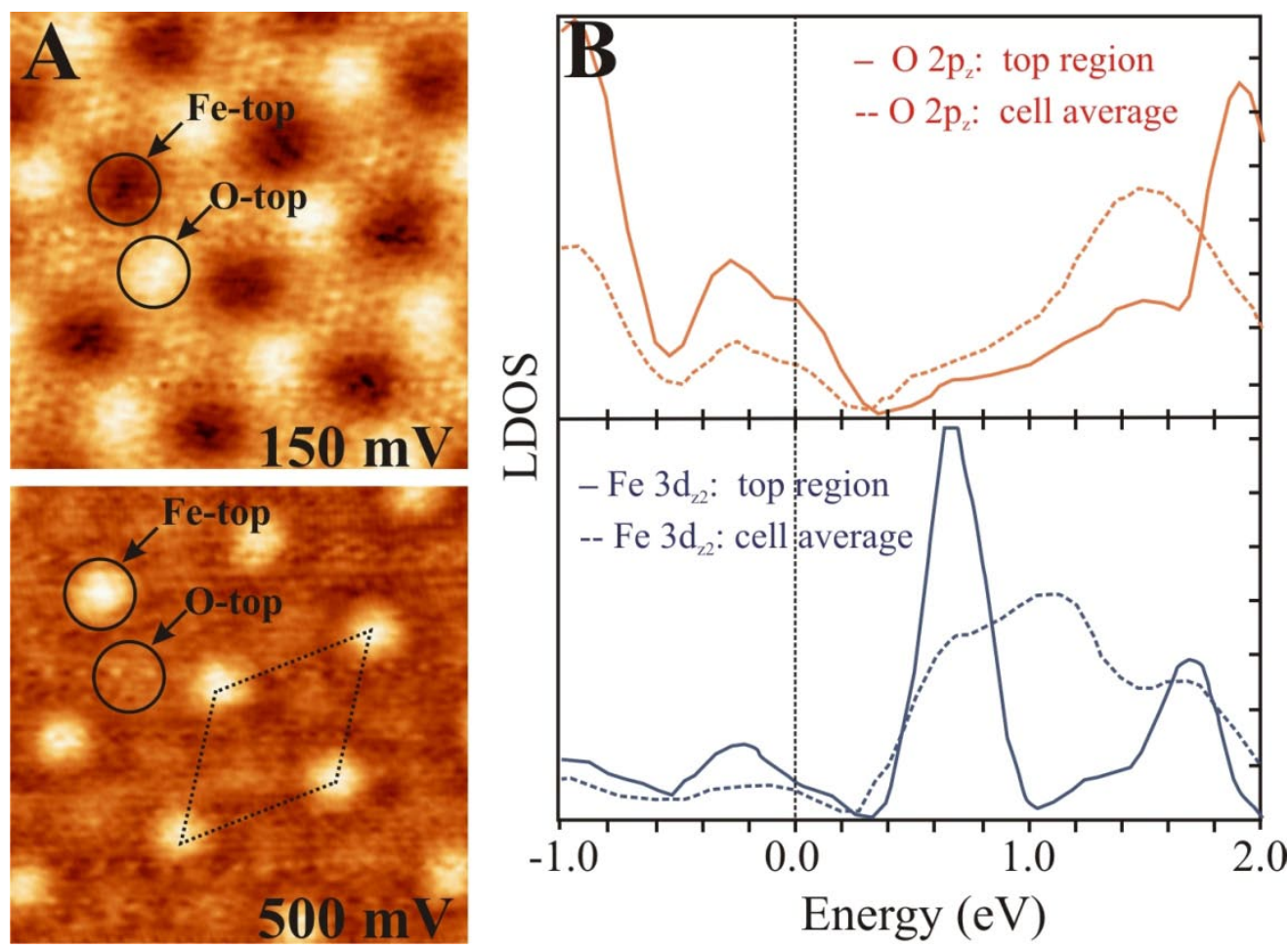

Fig. 3.4: (A) STM images of the FeO/Pt(111) coincidence structure taken at two different bias voltages $\left(7 \times 7 \mathrm{~nm}^{2}\right)$. The coincidence unit cell is marked by the dashed parallelogram. (B) Projected density of the $\mathrm{O}-2 p_{z}$ and $\mathrm{Fe}-\mathrm{d}_{z} 2$ states calculated for particular regions in the coincidence cell, as well as for the cell average. Regions with a particularly high LDOS due to interfacial hybridization appear bright in the STM images taken at the corresponding bias voltage. ${ }^{166}$

In the case of extremely strong interfacial coupling between metal and oxide support, the interaction might even lead to completely new film morphologies. Prominent examples for such a scenario are the various alumina films that can be prepared on NiAl surfaces of different crystallographic orientation, namely on $\mathrm{NiAl}(110),{ }^{155} \mathrm{NiAl}(001){ }^{161}$ and $\mathrm{Ni}_{3} \mathrm{Al}(111)$. ${ }^{160,175}$ In all three cases, the alumina forms complex unit cells, where the $\mathrm{Al}$ and $\mathrm{O}$ ions bind in different coordination schemes than in bulk corundum. Using DFT calculations, ${ }^{31,176}$ the strong interfacial coupling between $\mathrm{Ni}$ in the substrate and $\mathrm{Al}$ in the oxide film has been identified as the driving force for the atom arrangement at the interface. Details of this interplay shall be discussed for the alumina/NiAl(110) system in the following (Fig. 3.5). 


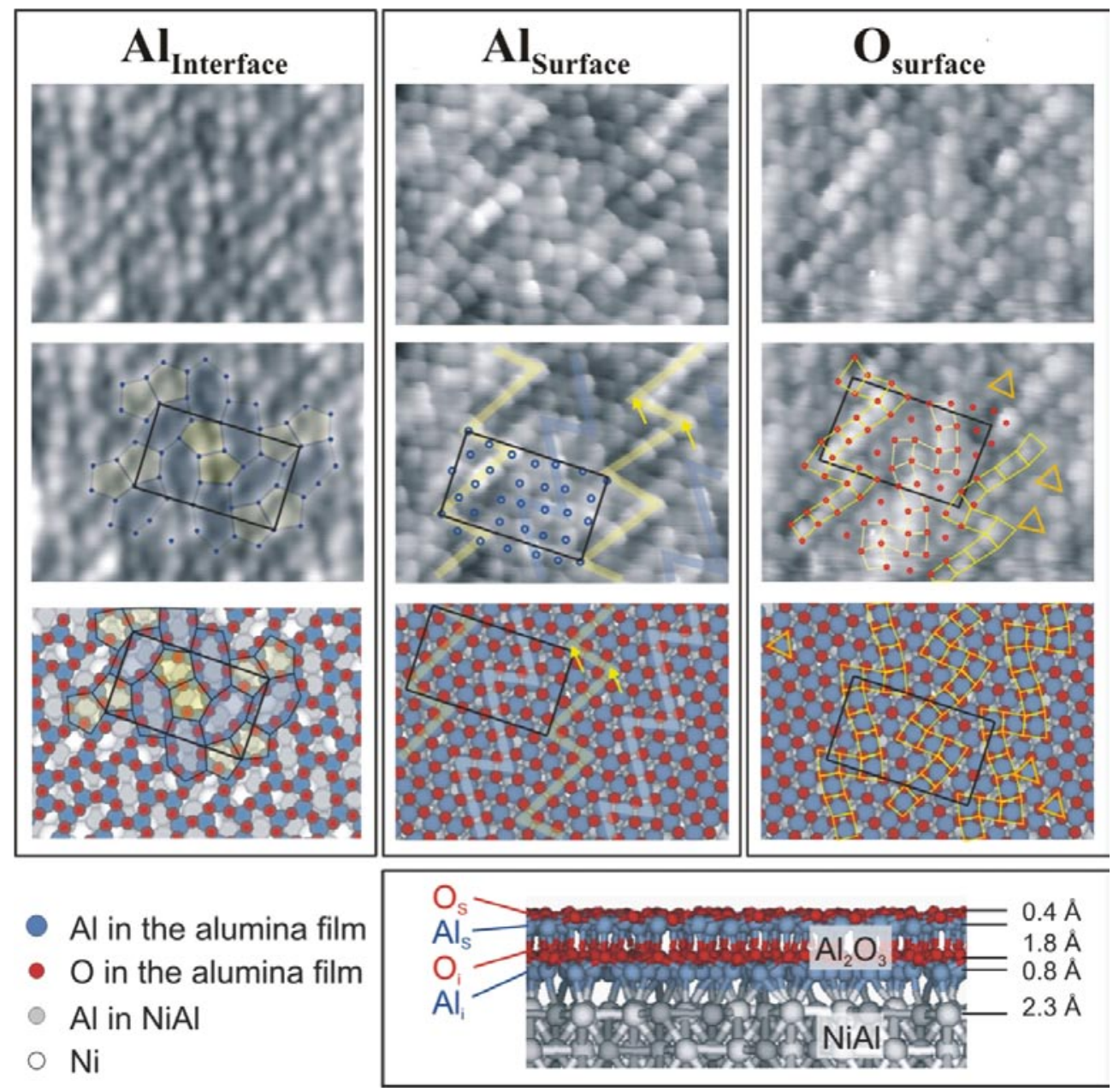

Fig. 3.5:

Atomically resolved STM images $\left(4.0 \times 2.9 \mathrm{~nm}^{2}\right)$ of three layers of the alumina/NiAl(110) film in comparison with a structure model taken from Ref.[31]. The Al ions at the interface form a network of heptagons and pentagons, which enables optimal overlap with the $\mathrm{Ni}$ atoms in the support. The hexagonal $\mathrm{O}_{\text {inter }}$ layer located above is not shown, because it cannot be resolved in the STM. The surface $\mathrm{Al}_{\mathrm{s}}$ plane contains five-fold (marked by the zig-zag lines) and four-fold coordinated $\mathrm{Al}^{3+}$ species that can be distinguished due to the higher LDOS and brighter STM appearance of the former ones. The $\mathrm{O}^{2-}$ ions in the surface $\mathrm{O}$-plane are finally arranged in squares and triangles, depending on their binding geometry with the $\mathrm{Al}_{\mathrm{s}}$ sitting below. A cut through the four oxide layers is shown in the bottom panel.

The film grows in a self-limiting regime and terminates after four layers of $0.5 \mathrm{~nm}$ total thickness. ${ }^{48}$ The interfacial $\mathrm{Al}_{\mathrm{i}}$ atoms arrange themselves in pentagonal and heptagonal rings on the bulk-like $\mathrm{NiAl}(110)$ surface and determine therewith the symmetry of the following planes (Fig. 3.5, left panel). ${ }^{31}$ The adjacent $\mathrm{O}_{\mathrm{i}}$ layer and the following surface $\mathrm{Al}_{\mathrm{s}}$ layer adapt both hexagonal atom arrangements and lie directly on top of each other (central panel). The terminating $\mathrm{O}_{\mathrm{s}}$ plane finally consists of oxygen triangles and squares with one $\mathrm{Al}_{\mathrm{s}}$ ion from the layer below located in the centre (right panel). This unusual lattice structure is dictated by two 
principles. (i) The overlap between the oxide $\mathrm{Al}_{\mathrm{i}}$ ions and the $\mathrm{Ni}$ atoms in the metal is maximized, as their interaction anchors the film to the support. The interfacial $\mathrm{Al}_{\mathrm{i}}$ species almost exclusively bind to the closed-packed $\mathrm{Ni}$ rows but not to the parallel Al rows in the $\mathrm{NiAl}$ surface, giving rise to the formation of the $\mathrm{Al}_{5}$ and $\mathrm{Al}_{7}$ rings. (ii) Each $\mathrm{O}$ ion has to be adjacent to three $\mathrm{Al}$ neighbors to maintain the same coordination and charge state as in bulk $\mathrm{Al}_{2} \mathrm{O}_{3}$. As the $\mathrm{O}_{\mathrm{i}}$ atoms in the lowest oxygen layer are already bound to two $\mathrm{Al}_{\mathrm{i}}$ ions in the interface plane, they can only coordinate a single $\mathrm{Al}_{\mathrm{s}}$ in the layer above. Consequently, the $\mathrm{O}_{\mathrm{i}}$ and the $\mathrm{Al}_{\mathrm{s}}$ planes need to be structurally identical and located on top of each other. Similarly, the surface $\mathrm{O}_{\mathrm{s}}$ layer is unable to adopt its preferred hexagonal symmetry but forms a complex pattern to coordinate the underlying $\mathrm{Al}_{\mathrm{s}}$ atoms in pyramidal (four $\mathrm{O}_{\mathrm{s}}$ above a central $\mathrm{Al}_{\mathrm{s}}$ ) as well as tetrahedral geometry (three $\mathrm{O}_{\mathrm{s}}$ above a central $\mathrm{Al}_{\mathrm{s}}$ ), in contrast to all known bulk corundum structures. On the other hand, the alternation of five- and four-fold coordinated $\mathrm{Al}$ ions is also found in the $\gamma-\mathrm{Al}_{2} \mathrm{O}_{3}$ bulk phase, where the $\mathrm{Al}$ ions occupy both, the octahedral and tetrahedral sites in the oxygen sub-lattice.

The peculiar structure of the alumina thin film is also revealed in its stoichiometry that corresponds to $\mathrm{Al}_{10} \mathrm{O}_{13}$ and is therefore oxygen deficient with respect to bulk $\mathrm{Al}_{2} \mathrm{O}_{3}$. The lower oxygen content is easily explained by the presence of interfacial $\mathrm{Al}_{\mathrm{i}}-\mathrm{Ni}$ bonds, saturating already some valences of the interfacial $\mathrm{Al}_{\mathrm{i}}$ atoms. On the basis of the two building principles discussed above, a chemically saturated and strongly-bound alumina ad-layer is formed on the $\mathrm{NiAl(110)}$ surface, which has no analogue to the bulk alumina phases. It shares however similarities to the oxide films grown on $\mathrm{Ni}_{3} \mathrm{Al}(111)^{176}$ and $\mathrm{NiAl}(001)$. In all three cases, atomically-resolved STM images of the different alumina planes played a decisive role for the elucidation of the complex film structures and provided the necessary input parameters for the DFT calculations that finally led to a valid structure model. ${ }^{31,177,178}$ It should be noted that diffraction methods failed in this particular example to identify the correct film structure. $^{179}$

Besides the alumina/NiAl system, other oxide films have been identified whose internal structure largely deviates from the known bulk oxide phases. Examples are the $\mathrm{Pd}_{5} \mathrm{O}_{4}$ film on $\operatorname{Pd}(111),{ }^{159,180}$ the $\mathrm{MnO}$ film on $\mathrm{Pt}(111)^{151}$ and the different $\mathrm{TiO}_{\mathrm{x}}$ structures developing on the Pt(111) surface. ${ }^{181,182}$ 


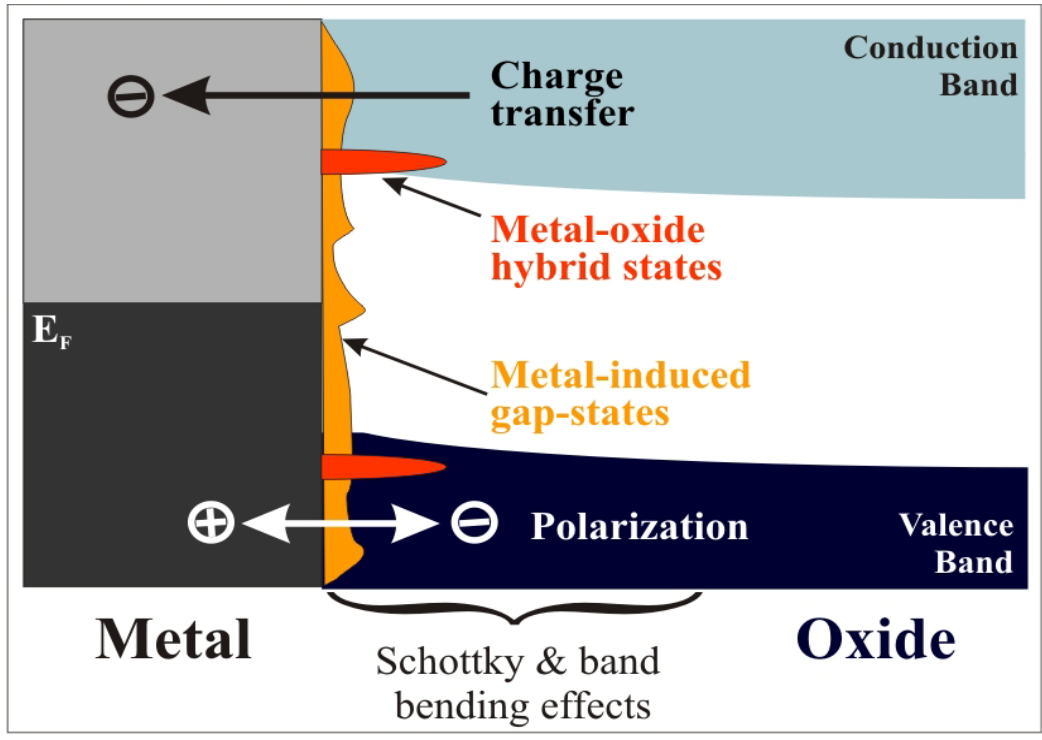

Fig. 3.6:

Potential diagram of a metal-oxide interface, depicting the different interaction mechanisms between the two electronic systems.

Apart from direct hybridization of states at the interface, the metal support affects the electronic properties of the oxide film in different ways (Fig 3.6). It introduces new states in the band gap of insulating oxides, resulting from the penetration of the metal wave functions into the oxide gap-region. Such metal-induced gap-states are characterized by a rather uniform state density throughout the gap and typically decay within several $\AA$ distance from the interface. ${ }^{162,183,184,185}$ Metal-induced gap-states and their availability even above the oxide surface are the basis for electron transport through insulating oxide films at low bias, but also contribute to the adsorption behavior of ultra-thin oxide films. ${ }^{186}$ The oxide electronic structure is further modified by the superior polarizability of the metal below, which has a direct influence on the metal-oxide adhesion. Pronounced polarization interactions occur especially for ionic oxides, as the large net-charges of the oxide ions introduce attractive forces with their image charges in the metal support. ${ }^{33,164,165}$ Polarization interactions are always connected to the formation of interfacial dipoles that in turn modify the work function of the system. The interfacial dipoles hereby increase, when charges are polarized away from the metal or are transferred into the oxide layer. This situation is met for $\mathrm{TiO}_{2}$ and $\mathrm{SiO}_{2}$ films on Mo supports and gives rise to an increase of the metal-oxide work function. ${ }^{187}$ In contrast, the work function decreases in absence of interfacial charge exchange because of two effects. First, the intrinsic dipole that naturally forms on every metal surface due to electron spill out is reduced by the oxide layer, which pushes the diffuse charge cloud back into the substrate. ${ }^{187}$ And second, attractive image-dipole interactions between electrons in front of the surface and 
their image charges in the metal are suppressed by the dielectric spacer layer, lowering the required energy to remove an electron from the system. Both oxide-induced effects might add up to a total work function decrease in the order of 1.0-1.5 eV, as calculated and measured for MgO films on $\mathrm{Mo}(001) .{ }^{187,188,189}$ Relative work-function changes can be accessed experimentally by conductance spectroscopy with the STM; a procedure that is demonstrated in detail in Chapter 3.6. $^{190}$
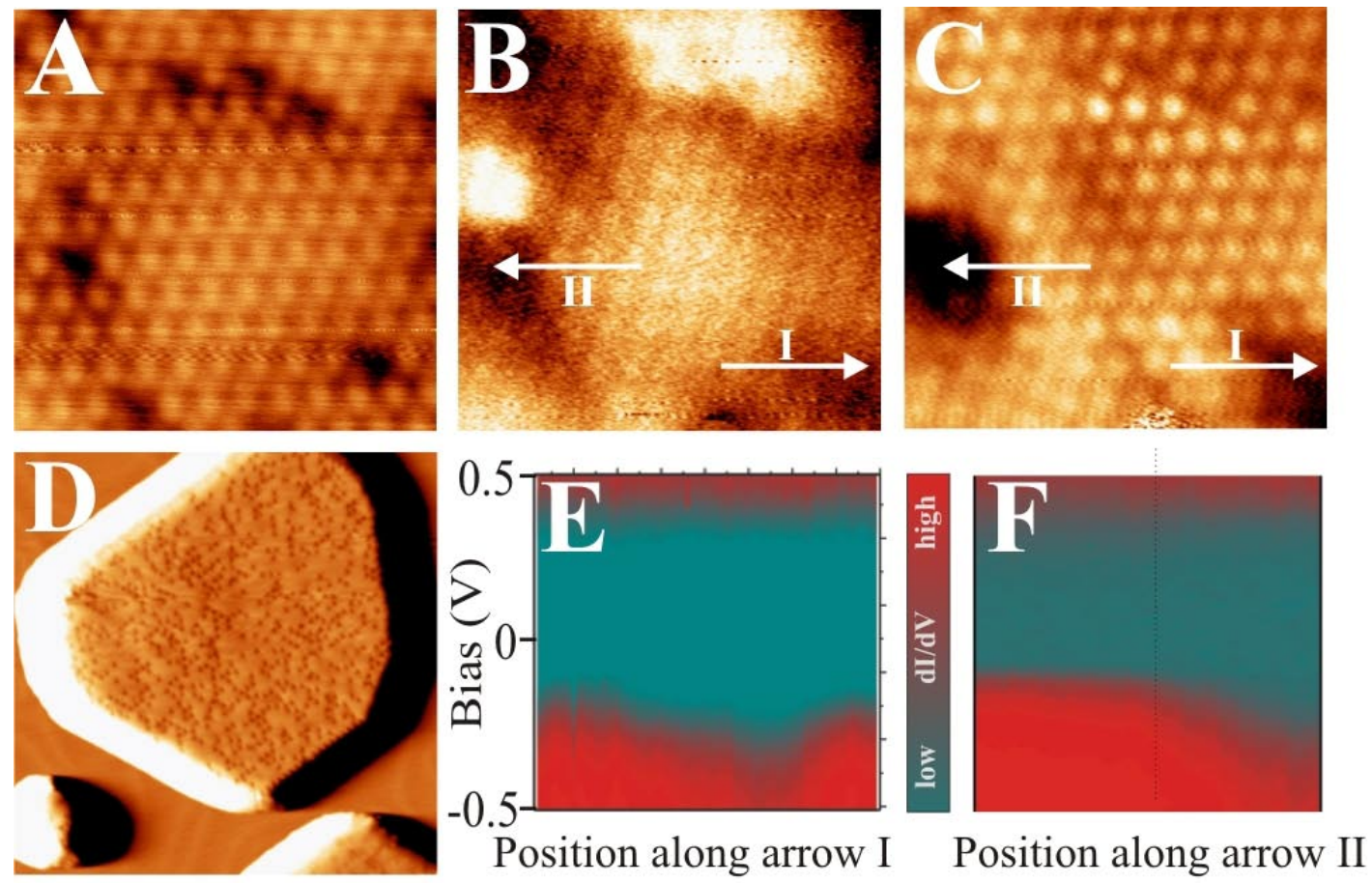

Fig. 3.7:

(A) STM topographic image of $\mathrm{V}_{2} \mathrm{O}_{3} / \mathrm{Au}(111)$ and corresponding conductance maps taken at (B) 240 $\mathrm{mV}$ and $(\mathbf{C})-240 \mathrm{mV}\left(5.5 \times 5.5 \mathrm{~nm}^{2}\right)$. The white arrows mark the course of the two series of $\mathrm{d} / \mathrm{dV}$ spectra shown in (E) and (F). In this matrix representation, the red and turquoise colors represent high and low $\mathrm{dI} / \mathrm{dV}$ intensity, respectively, and the turquoise region therefore marks the $\mathrm{V}_{2} \mathrm{O}_{3}$ band gap. Local gap modulations are due to changes in the oxide stoichiometry. (D) Overview STM image (70 $\times$ $70 \mathrm{~nm}^{2}$ ) showing a single vanadia crystallite on the $\mathrm{Au}(111)$ surface. ${ }^{194}$

Closely related to polarization interactions and charge exchange is the bending of oxide bands at the interface, a behavior commonly known as Schottky response (Fig. 3.6). ${ }^{184,191}$ The amount of band bending and its spatial extension away from the interface depend on the ionization potential of the free oxide compared to the metal Fermi level and on the available charge density in the oxide to screen the band perturbation. ${ }^{85}$ For thin oxide films, the decay length of the band bending often exceeds the layer thickness, and valence and conduction bands are simply offset in energy. This phenomenon can be probed by conductance spectroscopy, measuring the onset energies of the oxide bands with respect to the zero-bias 
position. For many wide-gap oxides, e.g. $\mathrm{SiO}_{2} / \mathrm{Mo}(112),{ }^{192}$ alumina/NiAl(110) ${ }^{193}$ and $\mathrm{MgO} / \mathrm{Ag}(001),{ }^{143}$ the mid-gap position is found to be lower than the Fermi level of the system, suggesting a charge transfer into the film. For $\mathrm{V}_{2} \mathrm{O}_{3}$ on $\mathrm{Au}(111)$, on the other hand, modulations of the midgap position with respect to Au Fermi level are detected, which occur on a length of few nanometer and sometimes involve changes of the total gap size (Fig. 3.7). ${ }^{194}$ This experimental finding is compatible with a local switching between $p$-type and $n$ type conductance behavior of the oxide film, being triggered by the dominances of either vanadium or oxygen defects within spatially-confined regions of the oxide lattice. The resulting defect states in the gap are filled via electron/hole transfer from the Au support and introduce a bending of the oxide bands in response to the charging effect. ${ }^{194}$ From evaluating the position of the band edges with respect to the Au Fermi level, the amount of nonstoichiometry and the charge exchange across the interface can be approximated. A similar band bending effect has been found for alumina films on NiAl(110). ${ }^{195}$ Along line defects, excess electrons related to the oxygen-deficiency of the perturbed oxide lattice are transferred out of the film into the metal support. The resulting positive net charge in the alumina is counterbalanced by a downward bending of the alumina bands, as derived from $\mathrm{dI} / \mathrm{dV}$ spectroscopy and DFT calculations. ${ }^{193,195}$

In general, the electronic coupling across a metal-oxide interface influences not only the adhesion between oxide and support, but also the intrinsic electronic structure of the thin film.

\subsection{Growth and relaxation of oxide thin films}

The growth of thin oxide films is usually initiated at structural inhomogeneities on the metal surface. Metal step-edges play hereby a particular role for oxide nucleation, as the chemically unsaturated nature of the step atoms facilitates oxygen dissociation and attachment (Fig. 3.8A). ${ }^{180,196,197}$ However also surface reconstructions, e.g. the $\mathrm{Au}(111)$ herringbone reconstruction, may provide suitable sites for stabilizing the oxide nuclei (Fig. 3.8B). ${ }^{198}$ Wellresolved STM measurements obtained at low film coverage are extremely helpful to elucidate the oxide nucleation behavior on metal supports, as they reveal the nature and spatial distribution of the oxide nuclei on the surface. Especially in-situ STM measurements are advantageous in this context, where an initially clean metal surface is scanned in an oxygen ambience and at elevated temperature. (Fig. 3A) ${ }^{151,154}$ By this means, the evolution of the oxide system from an early nucleation stage towards a closed and compact film can be monitored in real time and with high spatial resolution. ${ }^{199}$ 

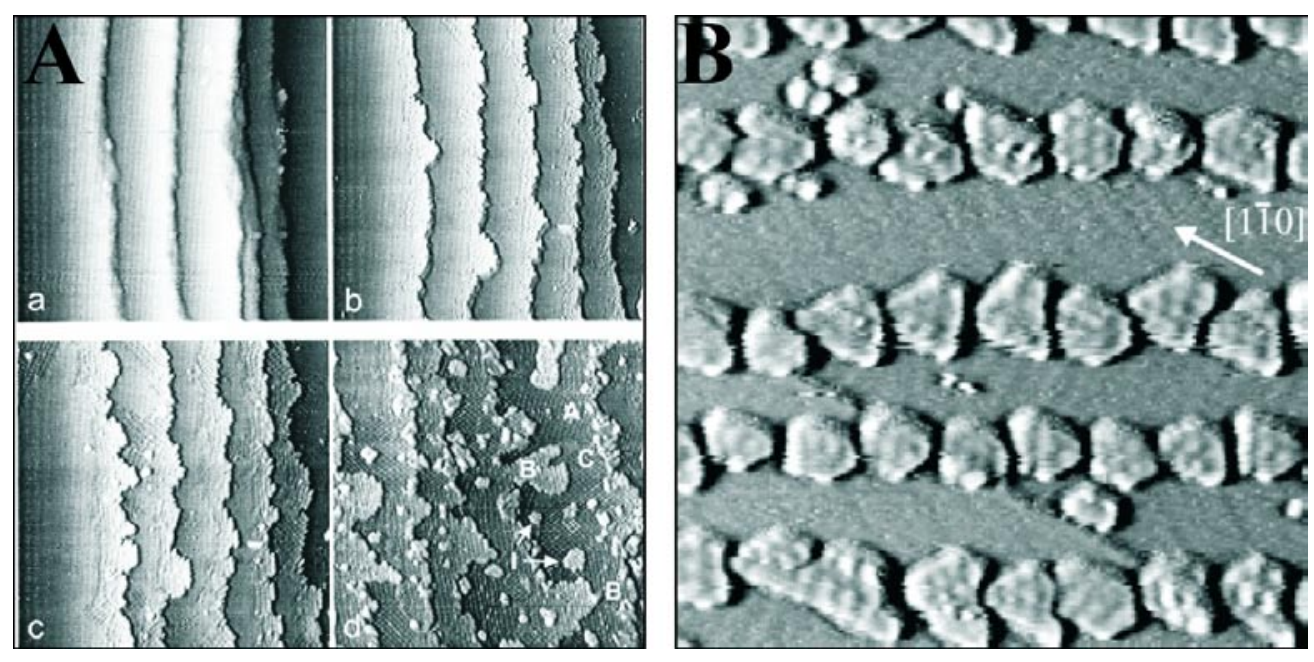

Fig. 3.8: (A) STM image series showing the gradual oxidation of a Ni(111) single crystal upon dosing 0 to 20 Langmuir of oxygen. ${ }^{154}$ The formation of $\mathrm{NiO}$ is initiated at the metal step edges and slowly proceeds towards the terraces $\left(50 \times 50 \mathrm{~nm}^{2}\right)$. (B) CoO islands grown on $\mathrm{Au}(111)\left(60 \times 60 \mathrm{~nm}^{2}\right)$. The spatial distribution of the oxide nuclei is given by the elbows of the Au herringbone reconstruction. ${ }^{198}$

With increasing film thickness, the oxide layer has to evolve from a transition regime, stabilized by the metal-oxide interactions, to a bulk-like oxide phase. Only for a small deviation between the metal and oxide lattice constants, the layer is able to adopt a pseudomorphic relationship with the substrate lattice. However, already a small lattice mismatch makes substantial elastic deformations necessary to stabilize the pseudomorphic growth of the oxide layer. The preparation of thick, but flat and crystalline films is therefore closely related to the ability of the oxide lattice to compensate growth-induced stress and to relax towards its intrinsic bulk parameters. Such relaxation processes of oxide films are often complex and may include dozens of atomic planes. In principle, many surface science techniques are able to monitor the structural relaxation of oxide materials; however STM measurements play a particular role as they provide real-space insights into the resulting surface morphology.

The amount of strain that builds up during film growth depends on the lattice mismatch between oxide and support, but equally important on the interfacial adhesion with respect to the lattice energies of the isolated systems. Consequently, pronounced lattice deformations only occur when the metal-oxide interaction is substantial, while incommensurate growth or the formation of a coincidence structure prevail otherwise (Fig. 3.9). ${ }^{146,147}$

Several mechanisms have been identified to release misfit-induced lattice strain in a metaloxide system. In the initial growth stage, elastic deformations in the more ductile of the two materials dominate the strain absorption. Elastic deformations are sufficient to compensate growth-induced strain as long as the deformation energy is smaller than the lattice energy. 
This is only the case for sufficiently lattice-matched systems of low thickness, e.g. for thin MgO films on $\mathrm{Ag}(001) .{ }^{142,143}$ For most metal-oxide combinations, however, structural rearrangements occur at a critical thickness to release the elastic deformations. The following mechanisms have been identified to reduce misfit-induced lattice strain in a metal-oxide system (Fig. 3.9): ${ }^{200}$

(i): Formation of misfit dislocations

(ii): Formation of tilted oxide planes (mosaics)

(iii):Stranksi-Krastanow growth associated with the formation of 3D oxide islands

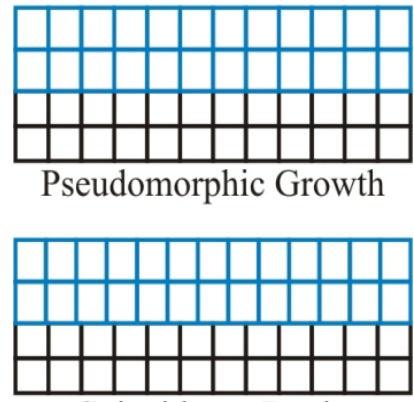

Coincidence Lattice

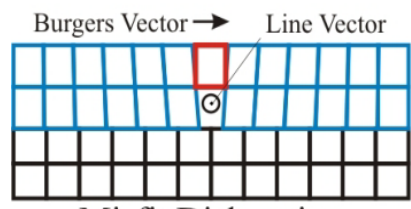

Misfit Dislocation

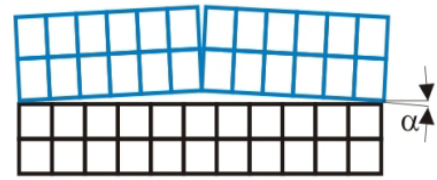

Mosaic Formation

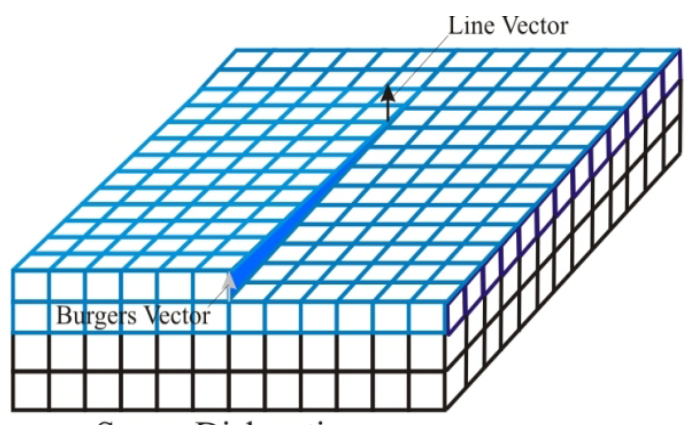

Screw Dislocation

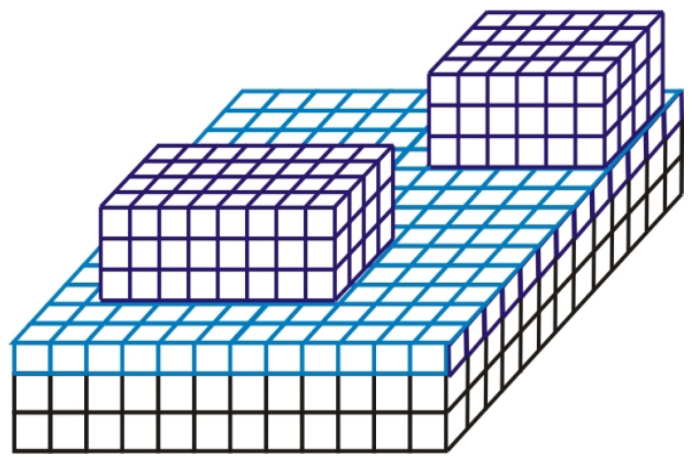

Stranski-Krastanov Growth

Fig. 3.9:

Different mechanisms to accommodate misfit-induced lattice strain and to enable structural relaxation of a thin oxide film.

(i) Misfit dislocations are extra atomic planes that have no counterpart in the lattice below and are inserted into oxide films with a smaller lattice constant than the support (Fig. 3.9). While around the dislocation, bonds to neighboring atoms are strongly compressed by the inserted plane, efficient release of the tensile strain takes place in between two dislocation lines. The strain distribution in the film becomes therefore inhomogeneous, which distinguishes elastic from inelastic deformations. When moving away from the metal-oxide interface that hosts the dislocation, the strain inhomogeneity fades away and the lattice parameter quickly homogenizes in the overlaying planes. In the thermodynamic equilibrium, misfit dislocations 
often form a regular network, whose periodicity is given by the energy balance between distorting the lattice and forming a dislocation line. ${ }^{201,202}$ This phenomenon is treated in the isotropic elasticity theory, ${ }^{203}$ which predicts not only the periodicity of the dislocation network but also the critical thickness, at which an insertion of dislocation lines becomes energetically favorable.

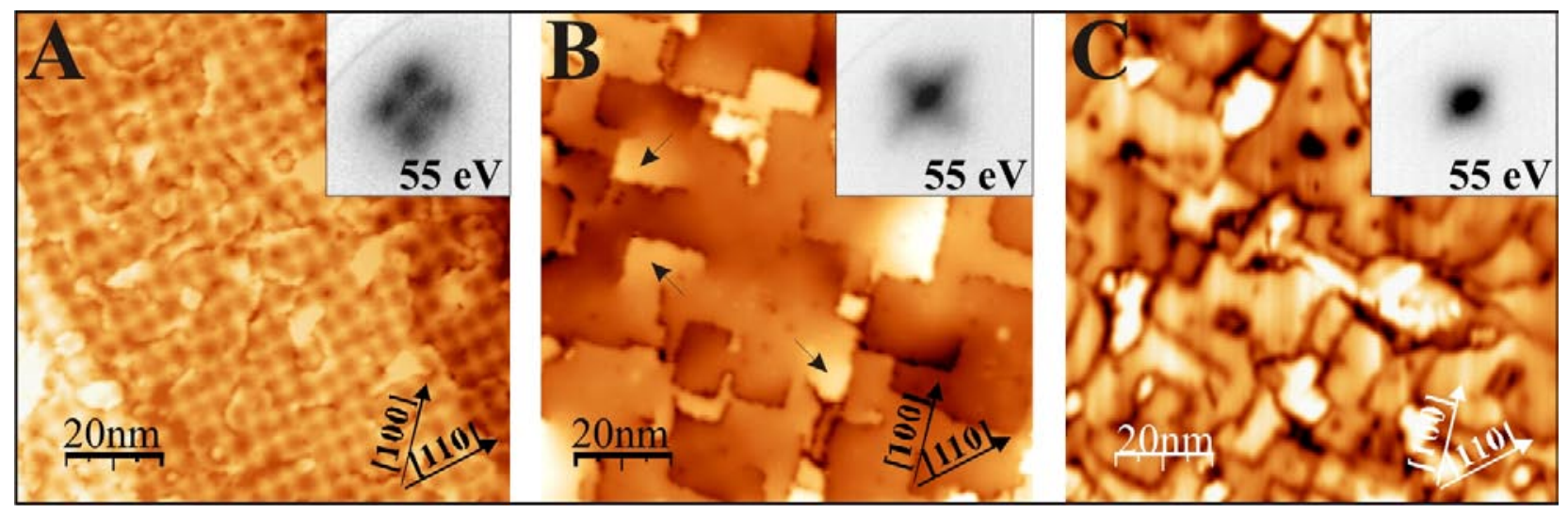

Fig. 3.10: STM topographic images and shape of the (1,0) LEED spot of MgO/Mo(001) films of (A) 2 ML, (B) $10 \mathrm{ML}$ and (C) $18 \mathrm{ML}$ thickness. The network of misfit-dislocations that releases approximately $70 \%$ of the total misfit strain is visible as the bright line pattern in (A). In (B), screw dislocations and mosaics (marked by the small arrows) are the structural elements that enable further lattice relaxation. The fine-structure in the LEED spots in both cases reflects the presence of $\mathrm{MgO}$ mosaics, tilted along the MgO[100] direction in (A) and along the [110] direction in (B). The thick film in (C) has essentially reached the bulk lattice constant of MgO, as manifested by simple, circular LEED reflexes. The blurred appearance of the STM image is due to the large film thickness that renders STM measurements difficult. ${ }^{51,152}$

Well-ordered networks of misfit dislocations have been observed in various thin-film systems, comprising metal, semiconductor and oxide over-layers. ${ }^{204,205}$ A particularly instructive example for a metal-oxide interface is the $\mathrm{MgO} / \mathrm{Mo}(001)$ system, whose relaxation characteristic has been investigated as a function of film thickness by STM, LEED and X-ray diffraction (Fig. 3.10). ${ }^{51,152}$ The STM hereby enables a simultaneous determination of the periodicity and crystallographic orientation of the dislocation lines, extending the information obtained from the diffraction methods. The $\mathrm{MgO} / \mathrm{Mo}$ lattice mismatch amounts to $-5.3 \%$, which implies that approximately $20 \mathrm{MgO}$ unit cells match 19 cells in the support. Accordingly, STM images of 1-4 ML thick MgO films show a regular network of dislocation lines with $55 \AA$ periodicity, being close to the computed value of $59.6 \AA$ for 19 Mo or 20 MgO lattice constants (Fig. 3.10A). Every bright line in the picture marks the position of an inserted MgO plane that is not in registry with the Mo atoms underneath. The image contrast originates partly from a topographic effect, as the regular Mo-O bonds at the interface are replaced by slightly longer Mo-Mg bonds along the dislocation line (see discussion in Chapter 
3.2). Additionally, the dislocation lines exhibit a deviating electronic structure due to a lower amount of interfacial coupling, which also contributes to the image contrast. Similar dislocation networks have been observed for $\mathrm{NiO}$ on $\mathrm{Pd}(001)^{206}$ and $\mathrm{MgO}$ on $\mathrm{FeO}(001)^{207,208}$ via STM and for $\mathrm{MgO},{ }^{209} \mathrm{NiO},{ }^{142}$ and $\mathrm{CoO}^{210}$ on $\mathrm{Ag}(001)$ via LEED. The presence of ordered defect patterns often affects the adsorption behavior of oxide thin films, which are consequently used as a template for the self-assembled growth of metal nano-particles (see Chapter $\underline{4.4 .4})^{211,212}$

(ii) Misfit-induced defects in strained oxide films can also take the form of screw dislocations. Screw dislocations are characterized by a parallel alignment of the dislocation vector that marks the direction of the inserted plane, and the Burgers vector that is the difference vector between a closed loop through the crystal lattice with and without dislocation (see Fig. 3.9). ${ }^{200,213}$ In contrast, both vectors are perpendicular to each other in edge dislocations, which is the general term for the line defects discussed in (i). For the MgO film on $\mathrm{Mo}(001)$, the formation of screw dislocations is observed as a competing relaxation mechanism to the insertion of the dislocation network. ${ }^{51,152}$ Their tentative origin lies in the coalescence of neighboring $\mathrm{MgO}$ islands on the Mo surface with an out-of-phase lattice relation. The position, at which the core of a screw dislocation penetrates the surface, is easily recognized in STM topographic images, as it is marked by the emergence of a new step edge (Fig. 3.10B).

Screw dislocations are inherently connected with the development of mosaics, comprising small oxide patches that are misaligned by some degrees against the global surface plane (Fig. 3.9). ${ }^{200}$ Mosaic formation represents another means to release misfit-induced strain, because it permits a slight expansion of the inter-atomic spacing in tilted compared to flat surface regions. The corresponding tilt angles can, in principle, be deduced from STM topographic images, e.g. by measuring the upward slope of a tilted plane against the global surface orientation. However, more accurate values are obtained with diffraction methods. Here, surface mosaicity leads to a characteristic satellite structure around the fundamental reflexes, from which the tilt angles can be deduced (see inset in Fig. 3.10B). ${ }^{214}$ The formation of tilted oxide-planes is a typical phenomenon occurring during film relaxation and has been observed in real space for $\mathrm{MgO} / \mathrm{Mo}(001)^{152}$ and $\mathrm{NiO} / \mathrm{Ni}(100)^{215}$ and in reciprocal space for $\mathrm{MgO} / \mathrm{Ag}(001)^{141,142}$ and $\mathrm{Pd} / \mathrm{MgO}(001){ }^{216}$ 


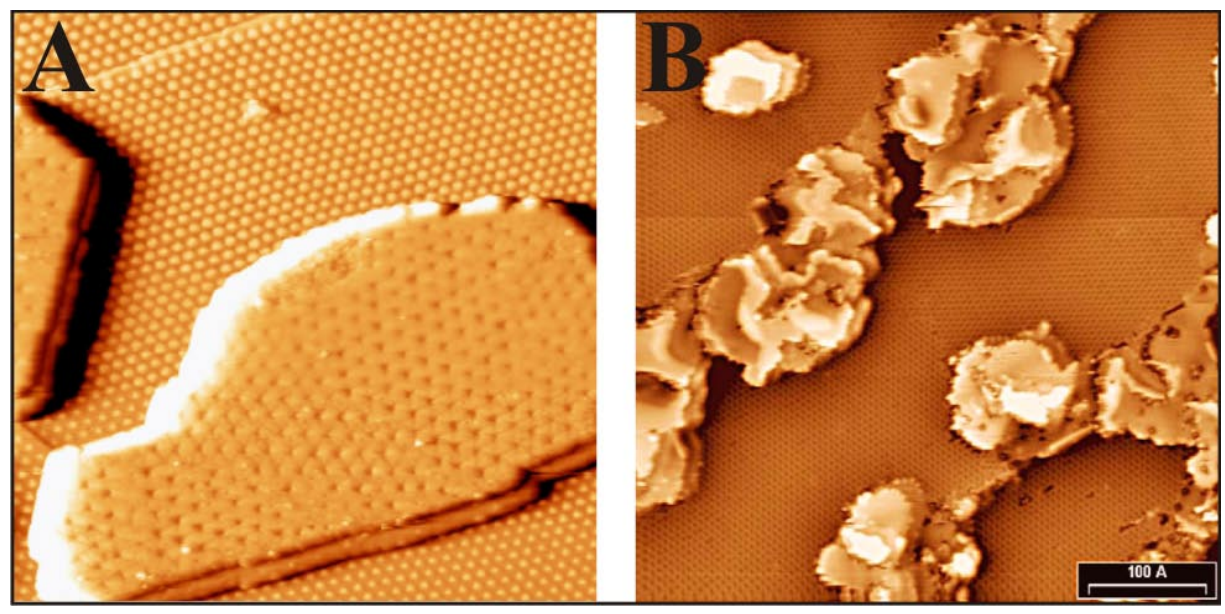

Fig. 3.11: Examples for the Stranski-Krastanov growth of oxides: (A) $\mathrm{FeO}$ wetting layer and 3D islands of $\mathrm{Fe}_{1-\mathrm{x}} \mathrm{O}$ grown on $\mathrm{Pt}(111)\left(100 \times 100 \mathrm{~nm}^{2}, 4.5 \mathrm{~V}\right)$ and $(\mathbf{B})(4 \times 2)$ surface oxide and bulk-like $\mathrm{NiO}$ islands on $\mathrm{Pd}(001)\left(50 \times 50 \mathrm{~nm}^{2},-2.0 \mathrm{~V}\right) .{ }^{167}$

(iii) The limiting case of film growth is reached, when strain relaxation cannot be realized in the complete film any more, but only in isolated three-dimensional oxide islands. ${ }^{153,200,217}$ Even in this case, the first oxide layer might wet the support, as the required deformation energy is compensated by the substantial gain in surface-free-energy when covering a metal surface with an oxide film. However, already in the second or third layer, the misfit strain is not stabilized in the oxide lattice any more, and the film breaks open into an array of isolated oxide islands (Fig. 3.9). The resulting growth regime is known as Stranski-Krastanov mode and has been observed for $\mathrm{Fe}_{1-\mathrm{x}} \mathrm{O}$ on $\mathrm{Pt}(111)^{28,147}$ as well as for $\mathrm{NiO}$ on $\operatorname{Pt}(111)^{150}$ and $\operatorname{Pd}(001)^{167}$ (Fig. 3.11). It is predicted to occur on the polar $\mathrm{MgO} / \mathrm{Ag}(111)$ system as well. ${ }^{218}$

A special growth mode of thin oxide films on crystalline supports is the so called 'Carpet mode', which denotes the possibility to overgrow step edges in the substrate in the form of an elastic and continuous layer (Fig. 3.12). ${ }^{219}$ By this means, strong lattice distortions and repulsive forces between equally charged oxide ions at the step edge are suppressed, resulting in a decrease of the total energy of the system. The 'Carpet mode' is usually observed for metal-oxide systems with small adhesion, as the interfacial binding length needs to be increased at the low side of each substrate step. In the STM, this particular growth mode is best identified at sub-monolayer oxide coverage when substrate step edges are still discernable in the images. The 'Carpet mode' has been observed for $\mathrm{ZrO}_{2}$ islands on $\mathrm{Pt}(111)^{81}$ (Fig. 3.12) and thin CoO films on $\mathrm{Ag}(001)$ so far. ${ }^{220}$ 


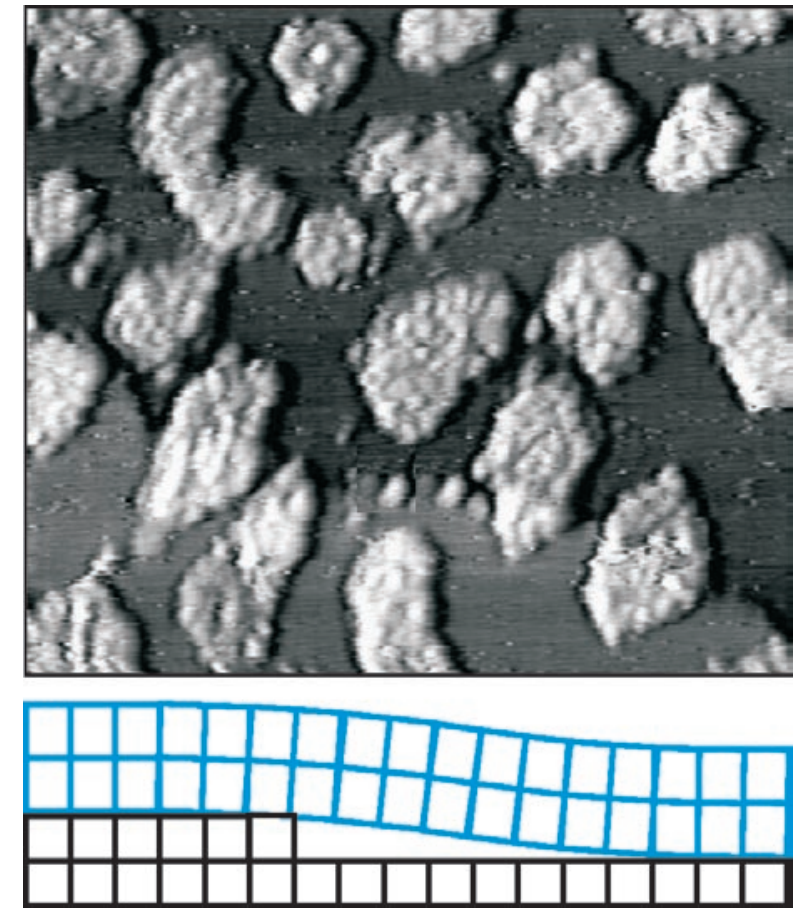

Fig. 3.12.

STM topographic image of $\mathrm{ZrO}_{2}$ islands on $\operatorname{Pt}(111)\left(75 \times 75 \mathrm{~nm}^{2}\right)$. The islands in the lower part of the image overgrow a Pt(111) step edge, a behavior denoted as 'Carpet-growth'. A structure model for this particular regime is sketched below. ${ }^{81}$

The dominant growth mode of oxide thin films on metal supports is not only determined by thermodynamic and crystallographic parameters, but additionally by the kinetics of film growth, e.g. by the flux and surface mobility of the incoming oxygen and metal atoms. Only for a good interplay between structural parameters, e.g. the lattice match at the interface, and thermodynamic growth conditions, the desired layer-by-layer growth of a flat and crystalline oxide films can be expected. In all growth stages, STM is a powerful tool to monitor the dimensionality and morphology of the resulting oxide film, but can also provide insights into the growth kinetics.

\subsection{Oxide films: Phase diagram and surface terminations}

Oxide thin films on metal supports can develop a large variety of different phases and surface configurations, whose number may considerably exceed the one of bulk oxides. The reason for this diversity is the stabilization of non-bulk configurations by the metal support, as well as the large number of potential growth parameters. The decisive requirement for the thermodynamic stability of a particular oxide phase is a minimum in the Gibbs free energy, determined as a function of oxygen partial pressure and temperature (viz. the $\mathrm{O}_{2}$ chemical potential). ${ }^{18,27}$ For thin oxide films, also the adhesion to the metal support plays a substantial role for this energy balance. ${ }^{50}$ The interrelation between the Gibbs energy of a particular oxide structure and the thermodynamic parameters is reflected in the phase diagram of the 
respective metal-oxygen system (see Fig. 3.14 for an example). For oxide films on metal supports, such phase diagrams are acquired with DFT calculations, and only verified at selected points by experimental, e.g. STM studies. ${ }^{26,171,221}$ Simple metals have usually a plain phase diagram, as only a few oxygen binding configurations can be stabilized (e.g. O-Mg binding geometries only in the rock-salt structure). Transition metals, on the other hand, may form a large number of oxide compounds that even coexist at similar thermodynamic conditions. ${ }^{18,29}$ This behavior reflects the small energy difference between single, double and multiple-charged cations in the case of transition metals. In general, the oxygen content of a particular oxide phase increases with the equilibrium $\mathrm{O}_{2}$ partial pressure, reflecting the thermodynamic stability of oxygen-rich structures. ${ }^{171,180}$ The upper limit of the oxygen-tometal ratio is hereby given by the number of valence electrons in the transition metal. For example, Ti can coordinate two oxygen atoms $\left(\mathrm{TiO}_{2}\right)$, as four $\mathrm{Ti}$ valence electrons are available to fill up the 2p shell of two oxygen atoms. In contrast, the oxygen content decreases with increasing temperature due to gradual decomposition of the compound. ${ }^{222}$ Understoichiometric oxide configurations can be formed with almost arbitrarily oxygen content.

The richness of the phase diagram of transition metal oxides becomes manifest in the different vanadium oxides. $^{29}$ The $\mathrm{V}$ atom can adapt a large number of oxidation states, ranging from $\mathrm{V}^{2+}$ in $\mathrm{VO}$ to $\mathrm{V}^{5+}$ in $\mathrm{V}_{2} \mathrm{O}_{5}$, and is able to coordinate oxygen in various geometries. Additionally, well-defined vanadia films form on metal supports with different symmetries and lattice constants, including Pd(111), Rh(111), W(110) and $\mathrm{Au}(111) .^{223,224,225} \mathrm{On} \operatorname{Pd}(111)$ for example, $226,227,228$ a hexagonal surface oxide with $\mathrm{V}$ atoms in tetrahedral coordination develops at monolayer coverage. With increasing metal exposure, the film transforms into a $\mathrm{VO}_{2}$ phase, and takes its final bulk-like $\mathrm{V}_{2} \mathrm{O}_{3}$ structure only above $3 \mathrm{ML}$ nominal thickness. ${ }^{223}$ The $\mathrm{V}_{2} \mathrm{O}_{5}$ phase, being the one with highest oxygen content, is stabilized only at $\mathrm{O}_{2}$ partial pressures exceeding 1 mbar. $^{229}$

A similar diversity of surface structures is produced by reducing an oxide film, as realized either via hydrogen exposure, electron bombardment or heating to elevated temperatures. $^{222,230,231}$ Thin-film vanadia on Rh(111), for example, undergoes several phase transition upon reduction, transforming from a $\mathrm{VO}_{2}$ into a $\mathrm{V}_{2} \mathrm{O}_{3}$-like structure with one and $0.5 \mathrm{~V}=\mathrm{O}$ groups per surface $\mathrm{V}$ atom, respectively. In higher reduction stages, different surface oxides without $\mathrm{V}=\mathrm{O}$ groups develop until finally a plain VO layer is formed (Fig. 3.13). 

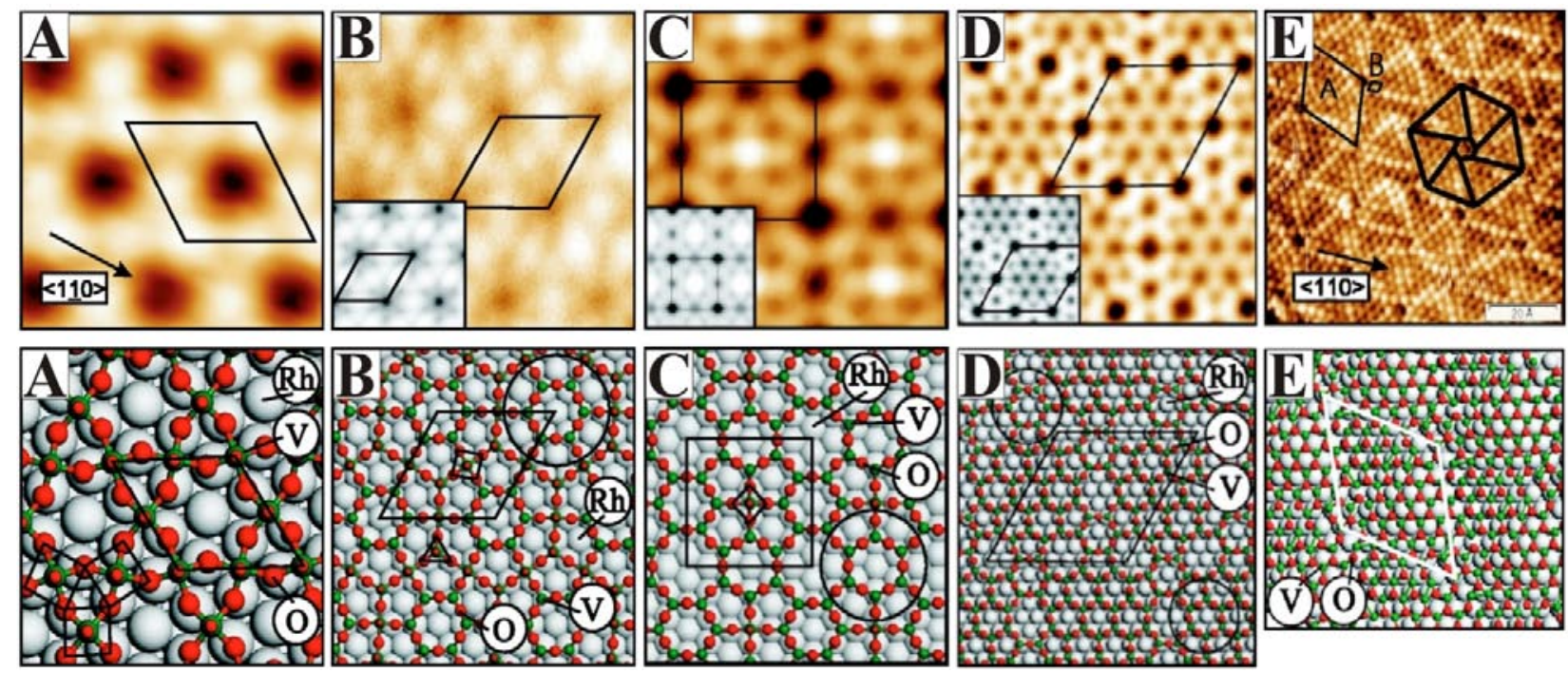

Fig. 3.13: STM topographic images and structure models for different vanadia phases on $\mathrm{Rh}(111)$, produced by a continuous reduction of the oxide film. In the model, red and green spheres mark the oxygen and vanadium atoms. (A) $\mathrm{V}_{3} \mathrm{O}_{9}$ phase consisting of $\mathrm{O}_{4} \mathrm{~V}=\mathrm{O}$ building blocks ( $\mathrm{V}: \mathrm{O}$ ratio $-1: 3$ ) $\left(1.5 \times 1.5 \mathrm{~nm}^{2}\right)$. (B) $\mathrm{V}_{11} \mathrm{O}_{23}$ phase with a V:O ratio of 1:2.1 $\left(4 \times 4 \mathrm{~nm}^{2}\right) .\left(\right.$ C) $\mathrm{V}_{13} \mathrm{O}_{21}$ with a $\mathrm{V}: \mathrm{O}$ ratio of 1:1.6 $\left(3.3 \times 3.3 \mathrm{~nm}^{2}\right)$. (D) Surface $\mathrm{V}_{2} \mathrm{O}_{3}$ with a V:O ratio of $1: 1.5\left(6 \times 6 \mathrm{~nm}^{2}\right)$. (E) Wagon-wheel VO structure $\left(8 \times 8 \mathrm{~nm}^{2}\right)$. The latter two oxide phases have no $\mathrm{V}=\mathrm{O}$ groups any more. ${ }^{222}$

A comparably complex behavior can be found for the rhodium oxides that are prepared by oxidation of the $\mathrm{Rh}(111)$ and $\mathrm{Rh}(110)$ surface with molecular and atomic oxygen. ${ }^{171,232,233}$ With increasing oxygen exposure, one-dimensional Rh-O rows with decreasing mutual distance appear on the $\mathrm{Rh}(110)$ surface and later transform into $\mathrm{Rh}-\mathrm{O}_{2}$ stripes. In a strongly oxidizing environment, a compact oxide film develops that consists of dense-packed, hexagonal O-Rh-O planes stacked on-top of each other. ${ }^{171}$ For $\mathrm{NiO}$ on $\mathrm{Pt}(111)$, a reversible phase switching between an oxygen-poor $\mathrm{Ni}-\mathrm{O}(2 \times 2)$ and an oxygen-rich $(7 \times 1)$ phase has been observed when cycling the $\mathrm{O}_{2}$ partial pressure between $1 \times 10^{-6}$ and $2 \times 10^{-6}$ mbar. ${ }^{150}$ Phase transitions as a function of the $\mathrm{O}_{2}$ partial pressure are also observed for the Pd-O surface oxide formed on $\mathrm{Pd}(111)^{180}$ and, at oxygen pressures exceeding 1 mbar, for $\operatorname{Pt}(110){ }^{234,235}$

The various oxide phases are usually characterized by different electronic properties and might therefore be distinguished via their different gap values. This effect has been exploited to study the decomposition of $\mathrm{V}_{2} \mathrm{O}_{5}$ surface layers at $400 \mathrm{~K}$, using STM and conductance spectroscopy. The decay of the oxygen-rich $\mathrm{V}_{2} \mathrm{O}_{5}$ into a $\mathrm{V}_{6} \mathrm{O}_{13}$ intermediate structure and a final $\mathrm{V}_{2} \mathrm{O}_{3}$ phase is accompanied by the gradual closing of the oxide band gap. ${ }^{236} \mathrm{~A}$ mapping of the local gap-size with $\mathrm{dI} / \mathrm{dV}$ spectroscopy thus provides spatially resolved information on the phase transition and the associated electronic reorganization. 
It should be mentioned that the same thermodynamic considerations that determine the development of a particular oxide film are also responsible for the surface termination of bulk oxides and thick oxide layers. The stoichiometry and structure of their top layer reflects the desorption-adsorption equilibrium with the surrounding gas atmosphere, and consequently depends on the $\mathrm{O}_{2}$ partial pressure and temperature during surface preparation. The phase diagram of the (0001) $\mathrm{V}_{2} \mathrm{O}_{3}$ surface, shown in Fig. 3.14, demonstrates this interrelation between the $\mathrm{O}_{2}$ chemical potential and the oxygen content of the surface. ${ }^{26}$ At strongly reducing or oxidizing conditions, the $\mathrm{V}_{2} \mathrm{O}_{3}$ surface exposes either a bulk-like vanadium plane (left part of the phase diagram) or an oxygen plane (right part), respectively. At intermediate $\mathrm{O}_{2}$ partial pressures, a vanadyl $(\mathrm{V}=\mathrm{O})$ terminated surface develops with different densities of $\mathrm{V}=\mathrm{O}$ groups. ${ }^{224,225}$ Similarly, several terminations have been observed in STM for the (111) surface of bulk $\mathrm{Fe}_{3} \mathrm{O}_{4}$ by adjusting different oxygen pressures during preparation. ${ }^{237,238}$
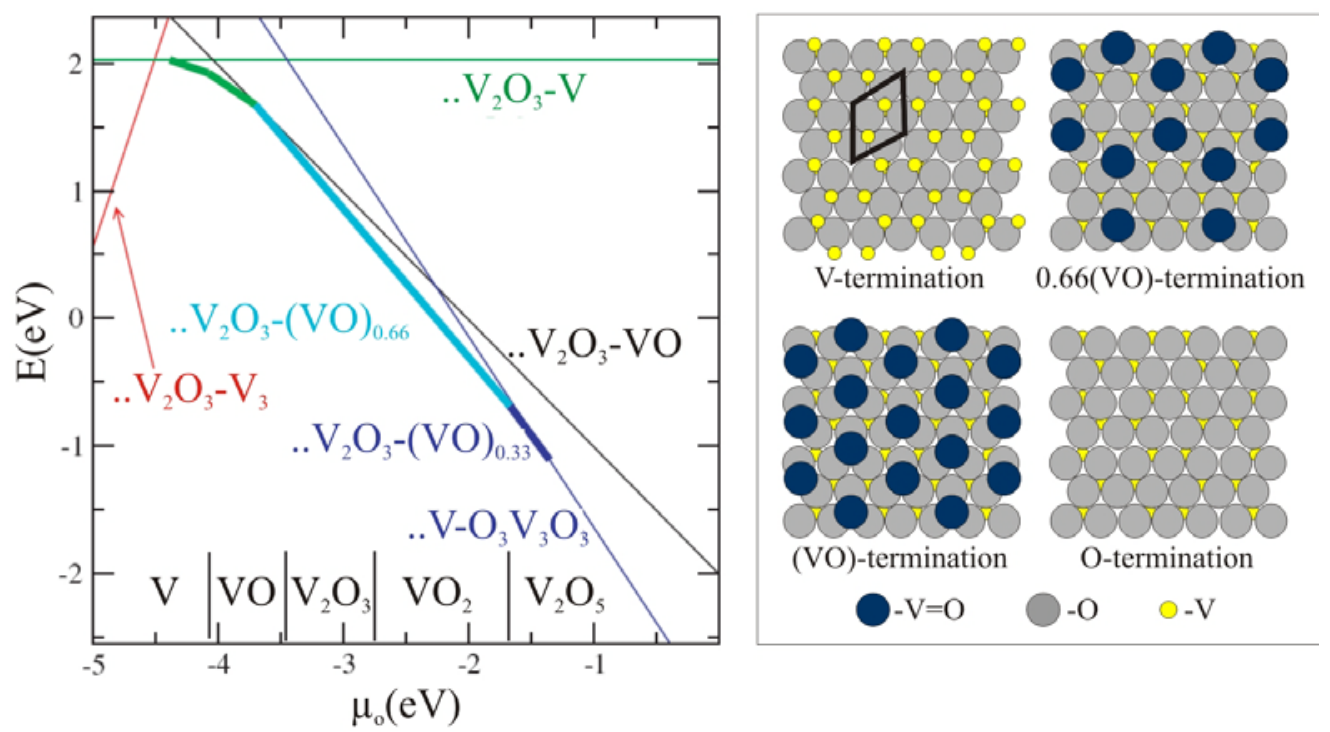

Fig. 3.14: Phase diagram showing the different terminations of a (0001)-oriented $\mathrm{V}_{2} \mathrm{O}_{3}$ bulk crystal as a function of the oxygen chemical potential $\mu_{\mathrm{O}}$. The surface energy per unit cell has been calculated by DFT. Thin and thick lines correspond to primitive and reconstructed cells, respectively. The stability regime of the corresponding bulk oxides is indicated at the bottom of the graph. ${ }^{26}$ Models of selected surface terminations are depicted on the right.

\subsection{Defects in the oxide surface}

The physical and chemical properties of oxide materials are inherently connected with the presence of defects in their lattice. The importance of defects with respect to metals relates to the strong electron localization and small screening ability of most oxide compounds as well as to the high degree of bond saturation in the ideal oxide surface. In wide-gap materials, defects often introduce localized states in the gap, which give rise to new optical excitations ${ }^{24}$ 
and may alter the bulk conductivity. ${ }^{18,40}$ Surface defects, on the other hand, offer an attractive binding environment for adsorbates and therefore govern the nucleation and growth behavior as well as the chemical reactivity of the oxide surface. ${ }^{27,36,37}$

Oxygen vacancies are the most common defect type and can be roughly divided into two groups. In highly-ionic oxide materials, the removal of an oxygen atom leaves behind two electrons per vacancy, which had filled the $O 2 p$ orbitals in the unperturbed lattice. ${ }^{23,55}$ These extra electrons occupy spatially-confined defect states localized at the vacancy site. A doubly occupied lattice defect is termed $\mathrm{F}^{0}$ (F stands for 'Farb' or color center and refers to its optical activity), while singly occupied and unoccupied vacancies are termed $\mathrm{F}^{+}$and $\mathrm{F}^{++}$centers, respectively. ${ }^{21,23}$ In reducible oxides, on the other hand, the electrons left behind after oxygen removal distribute among the next neighbor and second-next neighbor cations that in turn lower their oxidation state. Typical examples for this defect type are the oxygen vacancies in $\mathrm{TiO}_{2}{ }^{40,239} \mathrm{CeO}_{2}{ }^{76,240}$ and different vanadium oxides. ${ }^{241}$ The reduction of the oxide material can be followed by the emergence of cationic species in a reduced oxidation state using electron spectroscopy (e.g. $\mathrm{Ti}^{3+}$ or $\mathrm{Ce}^{3+}$ ions in addition to the $4+$ bulk species). ${ }^{242}$

Structural inhomogeneities in the surface form an equally important class of defects that comprises step edges, kinks and corner sites in the top-layer. In analogy to point defects, they expose low-coordinated and chemically unsaturated atoms, which govern the oxide adsorption behavior when the concentration of point defects is low. Furthermore, under-coordinated surface sites are characterized by a smaller Madelung potential with respect to the bulk lattice positions, as a certain quantity of then lattice neighbors is missing. A reduced Madelung potential now decreases the local gap size at the surface. As a result, surface steps, corners and kinks are the preferred trapping and recombination centers for coupled electron-hole pairs (excitons), being the fundamental electronic excitation in a gap material. ${ }^{23,55,243}$ The decay of excitonic modes is the main source for the emission of photons ${ }^{131,132,133}$ and hot-electrons from the oxide surface. ${ }^{244,245,246}$ Not surprisingly, the properties and abundance of lowcoordinated surface sites is therefore tightly connected with the optical and photochemical activity of oxide materials.

The major portion of the available knowledge on oxide defects has been produced by nonlocal spectroscopic techniques. Hereby, optical spectroscopy ${ }^{131,132,133}$, XPS and EELS ${ }^{247,248}$ are ideally suited to detect defect-induced electronic states in the band gap, while electronparamagnetic resonance spectroscopy (EPR) is sensitive to oxide defects carrying an unpaired 
electron. ${ }^{249}$ Thermal desorption and infrared-absorption spectroscopy, on the other hand, enable an investigation of defect-mediated adsorption processes. In contrast, the contribution of the STM to explore defect properties at the local scale is still relatively small. First attempts to characterize defects on the oxide surface exploited the fact that defects are able to anchor metal particles and thus become detectable in the STM via decoration effects (Fig. 3.15A). ${ }^{48,250,251,252,253}$ Using this approach, the spatial distribution and number density of defects has been revealed, however, no information on their nature and electronic properties could be obtained. The first topographic images of undecorated point defects were obtained on the $\mathrm{TiO}_{2}(110)$ surface, ${ }^{254,255}$ although those sites were confused with hydrogen adatoms for a long time due to their similar appearance. ${ }^{44,256}$ The final identification of the defects as oxygen vacancies came from water and oxygen adsorption experiments, which either led to an increasing number of $\mathrm{H}$ adatoms or to a decreasing concentration of $\mathrm{O}$ vacancies, respectively, and thus enabled the distinction of both species (Fig. 3.15B). ${ }^{257}$ The assignment was later verified by DFT calculations, which simulated the appearance of oxygen vacancies versus hydroxyl $(\mathrm{OH})$ groups in STM images taken at different conditions. ${ }^{258}$ Again on $\mathrm{TiO}_{2}(110)$, first attempts have been made to correlate structural and electronic properties of isolated defects using conductance spectroscopy with the STM. ${ }^{259}$ Those measurements succeeded in analyzing band-bending effects that were introduced by charged impurity-atoms in the subsurface region of the oxide material.
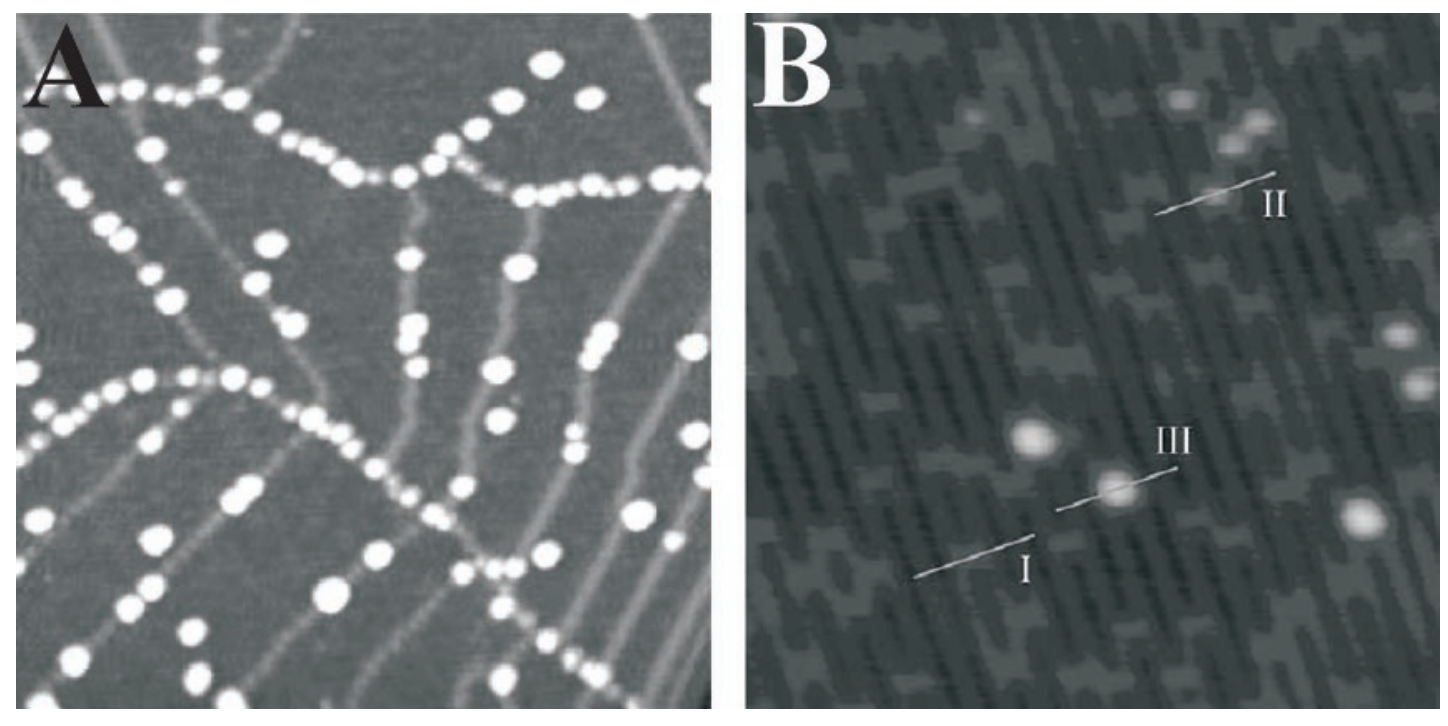

Fig. 3.15: (A) STM image displaying the decoration of line defects in an alumina/NiAl(110) film by Pd particles $\left(100 \times 100 \mathrm{~nm}^{2}\right){ }^{253} \mathbf{( B )}$ Single $\mathrm{O}$ vacancies (marked by I) in a slightly reduced $\mathrm{TiO}_{2}(110)$ surface $\left(16 \times 16 \mathrm{~nm}^{2}\right)$. The species (II) and (III) mark a single and a double hydroxyl group in the surface. $^{44}$ 
Point defects have been imaged on other bulk oxides as well, e.g. on $\mathrm{NiO}^{75}$ and $\mathrm{CeO}_{2}{ }^{76}$ The problem of the limited conductivity of most wide-gap oxides was solved in this case by performing the STM experiments at high temperature in order to install a sufficient carrier concentration in the oxide bands. On $\mathrm{NiO}(001)$, mainly $\mathrm{Ni}$ vacancies were observed, being identified by their characteristic contrast reversal in filled versus empty state images (Fig. 3.16). ${ }^{75}$ The $\mathrm{CeO}_{2}$ (111) surface, on the other hand, was found to expose a large number of single oxygen vacancies and vacancy clusters, whose concentration increased upon oxide reduction at $900^{\circ} \mathrm{C}$. Comparing the STM images to DFT calculations even enabled the identification of the under-stoichiometric $\mathrm{Ce}^{3+}$ ions, located in direct vicinity to the oxygen defects. $^{76}$
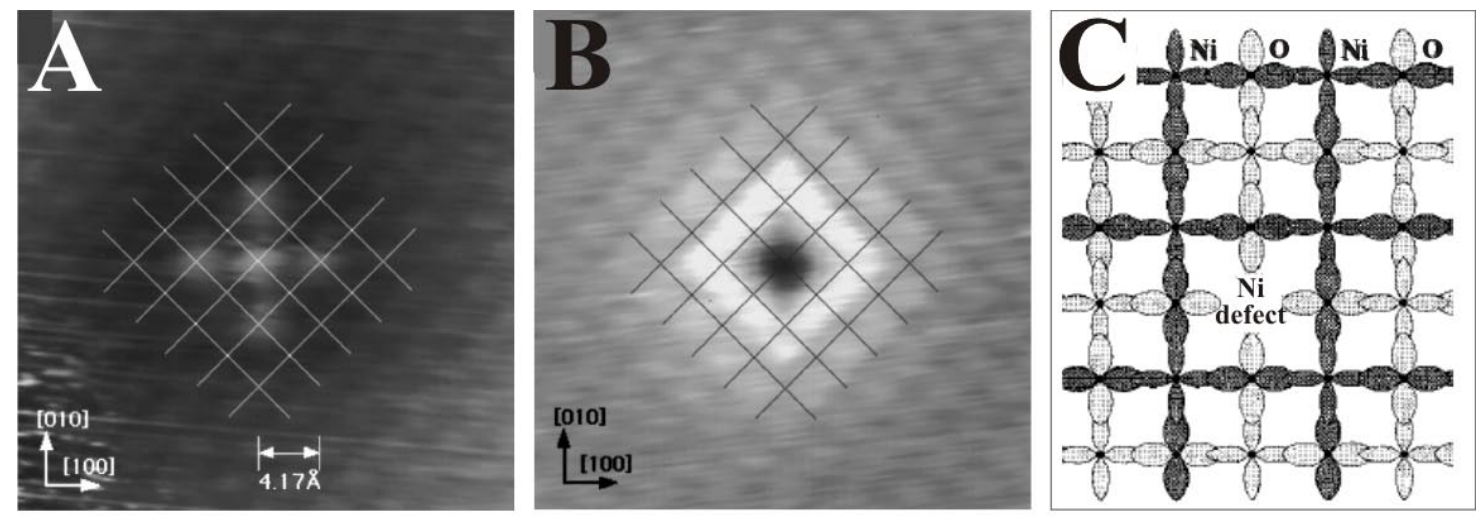

Fig. 3.16: (A) Empty $(+1.2 \mathrm{~V})$ and (B) filled-states (-1.3 V sample bias) STM images of a single Ni vacancy on $\mathrm{NiO}(001)$. The overlaid mesh denotes the $\mathrm{Ni}$ sub-lattice. In (A), the defect and four second-next neighbor Ni-atoms appear bright, while in (B) the surrounding $\mathrm{O}$ ions are imaged with enhance contrast. The bias-dependent contrast reversal has been rationalized by state density calculation of the Ni vacancy using the model shown in (C). ${ }^{75}$

Individual point defects were also imaged in different thin-film oxides grown on metal supports. ${ }^{77,260,261}$ In some cases, the similarity of their structural and electronic properties to the respective bulk defects could be proven, for instance for single $\mathrm{V}=\mathrm{O}$ vacancies in vanadylterminated film ${ }^{29,225,262}$ versus bulk $\mathrm{V}_{2} \mathrm{O}_{3}(0001)^{263}$ and for Fe and $\mathrm{O}$ defects in $\mathrm{Fe}_{3} \mathrm{O}_{4} / \mathrm{Pt}(111)$ films ${ }^{28,264}$ in comparison to the bulk oxide. ${ }^{237,265}$ A particularly comprehensive STM-analysis has been performed for point defects in thin MgO films grown on $\mathrm{Ag}(001){ }^{77,266}$ Using topographic imaging and conductance spectroscopy with the STM, two defect types could be distinguished on the surface (Fig. 3.17). Whereas the type-1 species are characterized by two $\mathrm{dI} / \mathrm{dV}$ peaks close to the Fermi level at $\pm 1.0 \mathrm{~V}$, type-2 defects exhibit a dI/dV maximum at 3.0 V. The bias position of the states in the band gap also determines their appearance in the STM images. Type-2 defects are imaged with bright contrast at $-3.5 \mathrm{~V}$, as tunneling out of the states is easy, whereas type- 1 defects are hardly visible at this condition. Based on DFT 
calculations for the level energies of different defects, the experimental species were assigned to positively-charged $\mathrm{F}^{+}$(type-2) and neutral $\mathrm{F}^{0}$ oxygen vacancies (type-1) in the MgO films. Both defect types could be transformed into one another by adding $\left(\mathrm{F}^{+} \rightarrow \mathrm{F}^{0}\right)$ or removing electrons from the defect states $\left(\mathrm{F}^{0} \rightarrow \mathrm{F}^{+}\right)$with a voltage pulse applied to the STM tip. Even the slightly deviating properties of oxygen vacancies located at terraces, step edges and corner sites could be probed thanks to high spatial resolution of the method (Fig. 3.17). ${ }^{23,77}$

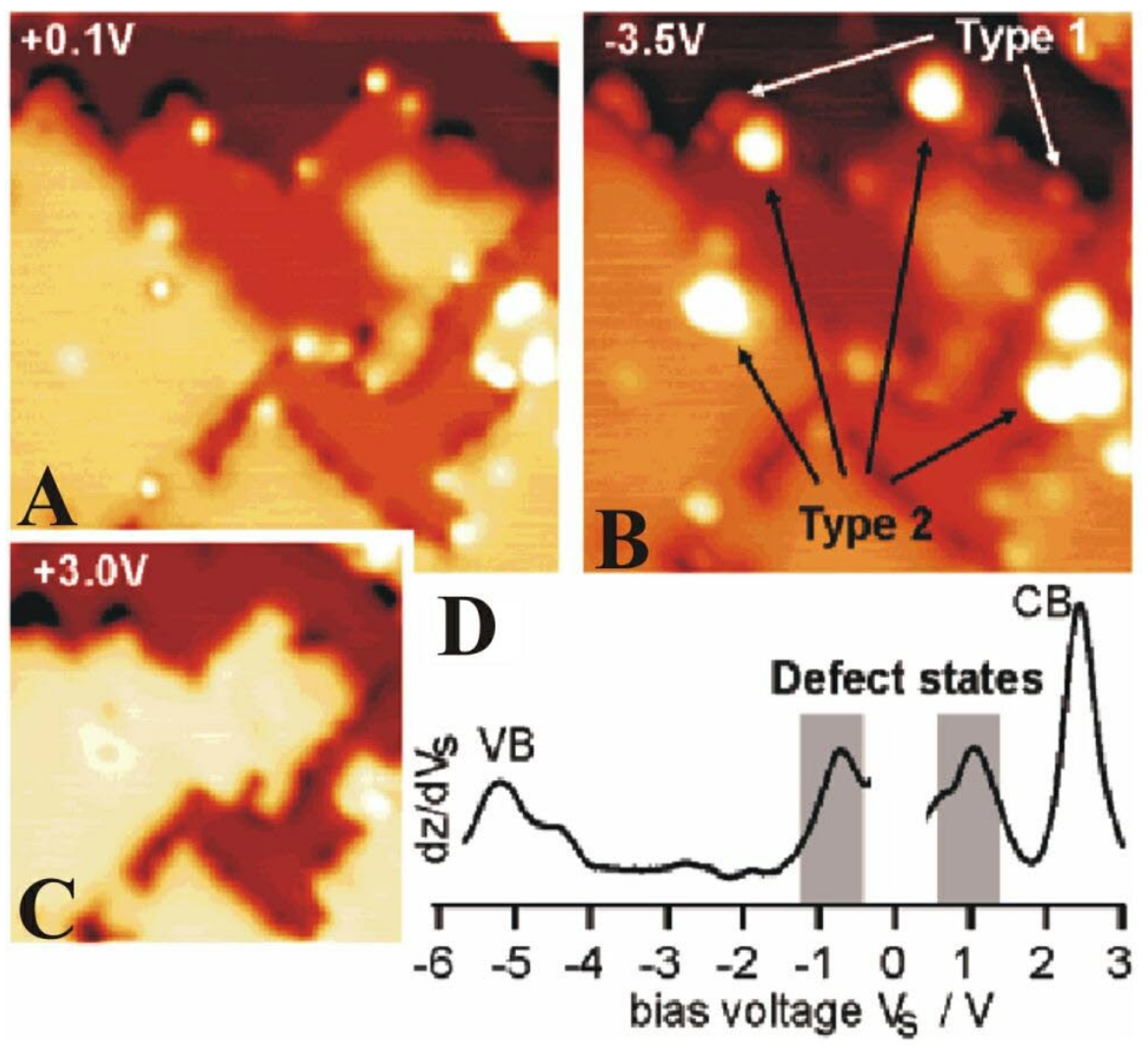

Fig. 3.17:

(A-C) Topographic STM images of 3ML MgO grown on $\mathrm{Ag}(001)(20 \times$ $20 \mathrm{~nm}^{2}$ ). While for bias values inside the band gap (A,B), defects appear as bright dots along the oxide step edges, only the film morphology is visible when tunneling into the $\mathrm{MgO}$ conduction band (C). Note that buried step edges in the Ag surface are only visible at certain bias conditions. (D) $\mathrm{dI} / \mathrm{dV}$ signature of a $\mathrm{F}^{0}$ center (type-1 defect) exhibiting an occupied and an empty state in the $\mathrm{MgO}$ band gap. $^{77}$

The STM has decisively contributed to the characterization of film-specific oxide defects too, which originate from the relaxation of misfit-induced lattice strain and have no counterpart in the respective bulk samples. Profound knowledge on such defects is not only required when using thin oxide films as model systems for bulk materials. It may also open possible routes to tailor thin-film properties by modifying their defect strucutre. ${ }^{53,197}$ Growth-induced defects are mostly line defects that means domain boundaries or dislocations, and have been identified in various thin oxide films, e.g. in alumina, ${ }^{177}$ magnesia, ${ }^{51}$ vanadia, $^{29}$ silica $^{221} \mathrm{CoO}^{210}$ and MnO. ${ }^{151}$ Due to their irregular arrangement and small number density, they are usually hard to detect with non-local diffraction and spectroscopic methods. The STM, on the other hand, succeeded in several cases to characterize such growth defects on an atomic level, providing structure models and the local stoichiometry around the defect line. This capability has been demonstrated for instance for antiphase-domain boundaries in alumina thin films on 
$\mathrm{NiAl}(110)$, where even an atomistic mechanism for the strain relaxation via dislocation lines was proposed (Fig. 3.18). ${ }^{178,195}$
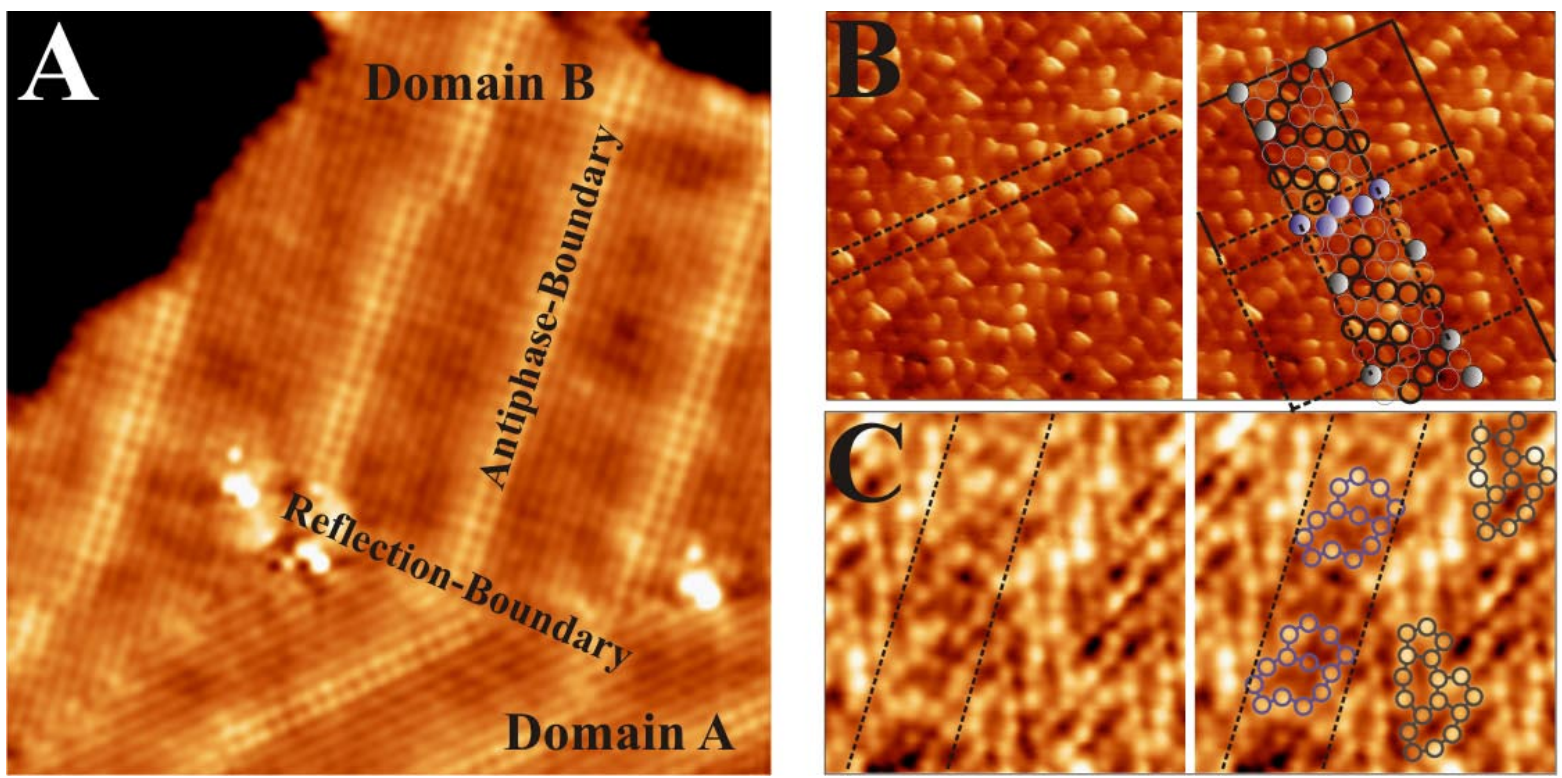

Fig. 3.18: (A) Alumina film on $\mathrm{NiAl}(110)$ with antiphase and reflection domain boundaries $(43 \times 43$ $\mathrm{nm}^{2}$ ). Whereas the former type separates two equally-oriented oxide domains and runs along defined crystallographic directions, the latter one forms between domains of different orientation and has no defined atomic structure. (B) Domain boundary between two A-domains with atomically resolved Al top layer $\left(4.6 \times 4.9 \mathrm{~nm}^{2}\right)$. The inserted $\mathrm{Al}$ row along the defect line is marked by blue spheres in the right image. (C) Domain boundary between two $\mathrm{B}$ domains showing the $\mathrm{Al}$ interface layer. Along the line defect, five and seven-membered $\mathrm{Al}$ rings are replaced by $\mathrm{Al}_{7}$ and $\mathrm{Al}_{9}$ configurations $(4.6 \times 4.6$ $\mathrm{nm}^{2}$ ). The modified regions in the oxide lattice are marked by dashed lines in (B, C).

In well prepared alumina films, antiphase-domain boundaries with defined orientations are inserted every 8-10 nm into the regular film (Fig. 3.18A). ${ }^{177}$ At the defect line, the rectangular oxide unit cell (see structure model in Fig. 3.5) is enlarged by $3 \AA$ along its long axis to allocate space for releasing the compressive strain induced by the lattice mismatch with the $\mathrm{NiAl}(110)$ support (Fig. 3.18B). To bridge this extra space, additional atoms are introduced along the line defect, whose positions have been determined for all four oxide planes with the help of atomically-resolved STM images and DFT calculations. ${ }^{195}$ In the interfacial $\mathrm{Al}_{\mathrm{i}}$ plane, the five and seven-membered $\mathrm{Al}_{i}$ rings of the unperturbed unit cell are replaced by $\mathrm{Al}_{7}$ and $\mathrm{Al}_{9}$ rings along the line defect (insertion of three $\mathrm{Al}_{\mathrm{i}}$ atoms), while four $\mathrm{Al}_{\mathrm{s}}$ atoms are introduced into the surface $\mathrm{Al}_{\mathrm{s}}$ layer directly at the boundary (Fig. $3.18 \mathrm{~B}, \mathrm{C}$ ). In accordance to the building principle of the alumina film explained in Chapter $\underline{3.2}$, the oxygen sub-lattice is replenished with four $\mathrm{O}_{\mathrm{i}}$ ions at the interface and another four $\mathrm{O}_{\mathrm{s}}$ ions in the surface plane. The resulting atomic structure along the line defect is oxygen deficient, as the local $\mathrm{O}$ to $\mathrm{Al}$ 
ratio decreases from $\mathrm{Al}_{40} \mathrm{O}_{52}$ (or $\mathrm{AlO}_{1.3}$ ) in the unperturbed alumina unit cell to $\mathrm{Al}_{47} \mathrm{O}_{60}$ $\left(\mathrm{AlO}_{1.27}\right)$ in the defect cell.

This under-stoichiometry induces a local modification of the oxide electronic structure. Along the domain boundaries, new unoccupied states appear in the oxide band gap that give rise to a strong bias-dependent contrast of the defect lines in empty-state STM images (Fig. 3.19). ${ }^{193}$ Whereas at low sample bias, the defects are nearly invisible due to their similar corrugation as the unperturbed oxide lattice, they show up as bright lines at higher positive bias. Pronounced contrast maxima with respect to the surrounding oxide patches occur at $+2.5,+3.5$ and $+4.5 \mathrm{~V}$ sample bias. At these bias positions, defect states are available in or near the oxide band gap, which enable efficient tunneling through the oxide film. The presence of these defect levels is verified by conductance spectroscopy with the STM (Fig. 3.19). In contrast to stoichiometric oxide patches, a pronounced peak at $+2.5 \mathrm{~V}$ shows up along the domain boundary, marking the lowest defect state. The higher states at 3.5 and $4.5 \mathrm{~V}$ are not resolved, as they lie above the conduction band onset at $3.1 \mathrm{~V}$ and are swamped by the in the conductance contribution through the oxide band. The high resolution of the conductance measurements provides also information on the spatial extension of the perturbed electronic structure around the line defects, or more precisely on the localization and symmetry of the underlying defect levels. Whereas the first defect state at $+2.5 \mathrm{~V}$ is imaged as single line running on top of the inserted atoms, a double strand with a ladder-like pattern appears for the second level at $+3.5 \mathrm{~V}$ (Fig. 3.19). ${ }^{193}$

The origin of the unoccupied defect states could be clarified in a subsequent DFT study. ${ }^{195}$ The missing $\mathrm{O}$ atom in each defect unit cell is expected to leave behind two extra electrons located in the vacancy site. In bulk alumina, the defect electrons fill Al-3s like states in the oxide band gap, forming a neutral $\mathrm{F}^{0}$ color center. ${ }^{267}$ In the thin alumina film, however, these electrons are transferred into the $\mathrm{NiAl}(110)$ support, as even the lowest defect state lies above the Fermi energy of the metal support. This charge transfer out of the oxide layer is counterbalanced by an electric field across the metal-oxide interface that induces a downward bending of the alumina bands. The observed increase of unoccupied state density around the line defects has therefore two origins: It results from the presence of unfilled defect levels related to the oxygen vacancies and reflects the down-shift of the alumina conduction band in response to the charge transfer into the metal. It is interesting to note that the oxygen-poor character of domain boundaries in alumina thin films makes them attractive for the nucleation of Rh and Pd particles ${ }^{195,253}$ (see Fig. 3.15) and for molecular adsorption. ${ }^{268}$. 

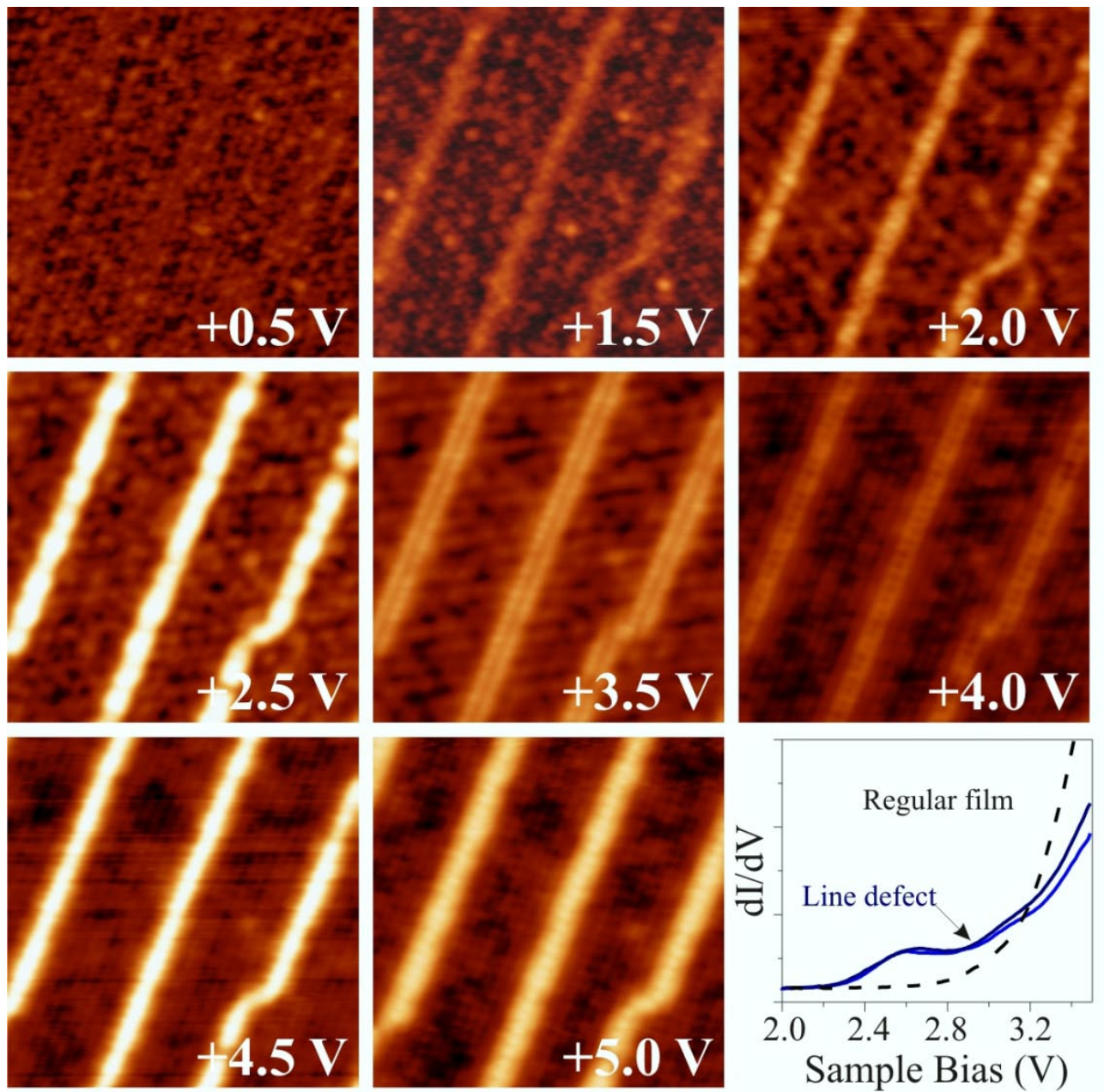

Sample Bias (V)

Fig. 3.19: Bias series showing several antiphase-domain boundaries in alumina/ $\mathrm{NiAl}(110)(30 \times 30$ $\mathrm{nm}^{2}$ ). The defect lines are nearly invisible at low imaging bias, but appear bright when the bias value matches a defect state in the oxide band gap (apparent for $+2.5,+3.5$ and $+4.5 \mathrm{~V}$ ). The right panel at the bottom depicts $\mathrm{dI} / \mathrm{dV}$ spectra taken on two defect lines and a stoichiometric oxide region. The first defect-induced gap state shows up as pronounced maximum at $+2.5 \mathrm{~V} .{ }^{193}$

The STM offers an additional means to study electronic properties of oxide defects, which is especially suited to explore local work-function changes introduced by structural inhomogeneities. The approach evaluates the electron-transport characteristic through the STM junction in the field-emission regime that means at bias voltages higher than the work function of the sample. ${ }^{97}$ At these conditions, the conductance through the oxide film is governed by field emission resonances (FER), which can be regarded as localized vacuum states confined by the sample surface on one side and the vacuum barrier on the other. A detailed introduction into the nature of FER will be given in Chapter 3.6. At this point, it is sufficient to note that the energy position of such resonances sensitively depends on the local work function, as this quantity sets the environment in which FER develop. ${ }^{269,270}$ Probing the 
first FER state on a regular versus a defect position in the oxide surface therefore yields information on the work function difference between both regions.

An example for this approach is given in Fig. 3.20A, which shows two FER spectra taken at a domain boundary and a defect-free region of a $10 \mathrm{ML}$ thick $\mathrm{MgO}$ film grown on $\mathrm{Mo}(001)$ (see also Fig. 3.10) ${ }^{82,188}$ Apparently, the first FER is shifted by $+0.75 \mathrm{eV}$ to higher energies at the line defect, indicating a substantial work function increase. Also the spatial distribution of the work-function can be mapped by this means, simply by imaging the oxide film at the bias position of the lowest FER (Fig. 3.20B). Whereas defect-free MgO patches appear with bright contrast due to the availability of the lowest FER for electron transport, the domain boundaries are imaged as dark network as the first FER is not yet reached there. The image contrast obtained in the field-emission mode is therefore dominated by work function modulations of the oxide surface, being emphasized by the FER-mediated transport channel. As expected, this particular contrast disappears in images taken in the tunneling regime $(\mathrm{U}<$ $5.0 \mathrm{~V}$ ), when the tip height is mostly controlled by topographic and electronic sample properties (Fig. 3.20C). The work-function increase observed along the domain boundaries in $\mathrm{MgO} / \mathrm{Mo}$ films is tentatively attributed to an abundance of negative excess charges at the defect lines (e.g. due to an oxygen surplus), which increases the local surface dipole and thus the barrier for electron emission. A comprehensive interpretation of the work-function variations would require the exact determination of the Mg-O ratio along the defect lines, and cannot be given at this point.
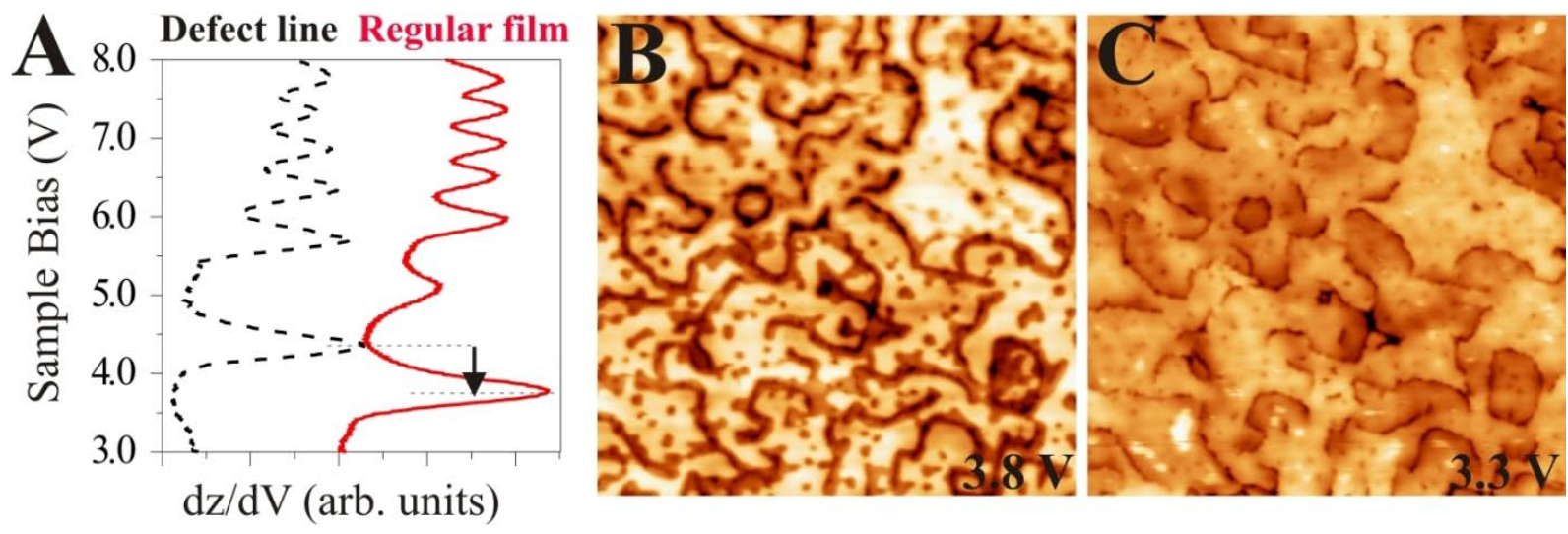

Fig. 3.20: (A) Dependence of the tip-height on the sample bias (dz/dV spectrum) measured on a defective and a regular position in the $\mathrm{MgO} / \mathrm{Mo}(001)$ surface. The $\mathrm{dz} / \mathrm{dV}$ maxima depict the positions of the FER, when electron transport through the tip-sample junction becomes particularly efficient. Due to a local increase of the work function, the FER are up-shifted around MgO line defects. STM images of $10 \mathrm{ML} \mathrm{MgO} / \mathrm{Mo}(001)$ taken at (B) the bias position of the first FER level and (C) below the lowest resonance $\left(100 \times 100 \mathrm{~nm}^{2}\right)$. Whereas the contrast in (B) is mainly governed by local work function modulations in the oxide surface, $(\mathrm{C})$ reveals to a first approximation the film morphology. 
In summary, the STM is a versatile technique to investigate defects in oxide surfaces, both from a topographic and an electronic point of view. Especially on well-prepared surfaces, defects may neither be accessed by diffraction techniques due to their random distribution, nor by conventional electron spectroscopy due to their low surface concentration. However, even a single oxygen vacancy can lead to a large perturbation of the differential conductance in the STM, for instance when the associated defect states are located inside the oxide band gap. The potential of the STM to probe individual oxide defects at the atomic scale is by far not exhausted, especially what a correlation between the atomic configuration and electronic structure of the defects concerns. Experiments on the adsorption behavior of oxide defects will be discussed in Chapter 4.4.4.

\subsection{Polar oxide films}

Polar oxides form a particularly interesting class of materials, as they possess a macroscopic surface dipole that largely determines the morphology, the electrostatic potential and the adsorption characteristic of the oxide surface. ${ }^{271}$ Oxide polarity arises from the stacking of atomic layers carrying an opposite net charge. In the case of non-compensated polarity, the dipole moments associated with each unit-slab consisting of anionic and cationic planes increases the total electrostatic energy, causing this quantity to diverge at infinite oxide thickness (Fig. 3.21). Materials of this kind belong to the type III polar surfaces according to the Tasker classification. ${ }^{272}$ Prominent examples are rocksalt and fluorites structures with (111) orientation, e.g. $\mathrm{MgO}(111), \mathrm{NiO}(111)$ and $\mathrm{CeO}_{2}(111)$, and the (0001) planes of wurzite, for instance $\mathrm{ZnO}(0001)$. The polar nature of other oxide structures is not intrinsic to a certain crystallographic orientation, but depends on the surface termination (Type II surfaces in the Tasker classification). For example, the Al-terminated $\mathrm{Al}_{2} \mathrm{O}_{3}(0001)$ is non-polar, as a chargecompensated unit cell that includes the surface layer can be constructed, while the oxygen termination introduces surface polarity. The same consideration holds for the O- (non-polar) versus the Ti-terminated (polar) $\mathrm{TiO}_{2}(110)$ surface. The occurrence of surface polarity therefore depends on both, the crystallographic orientation and the termination of the oxide surface. ${ }^{33,271}$ 

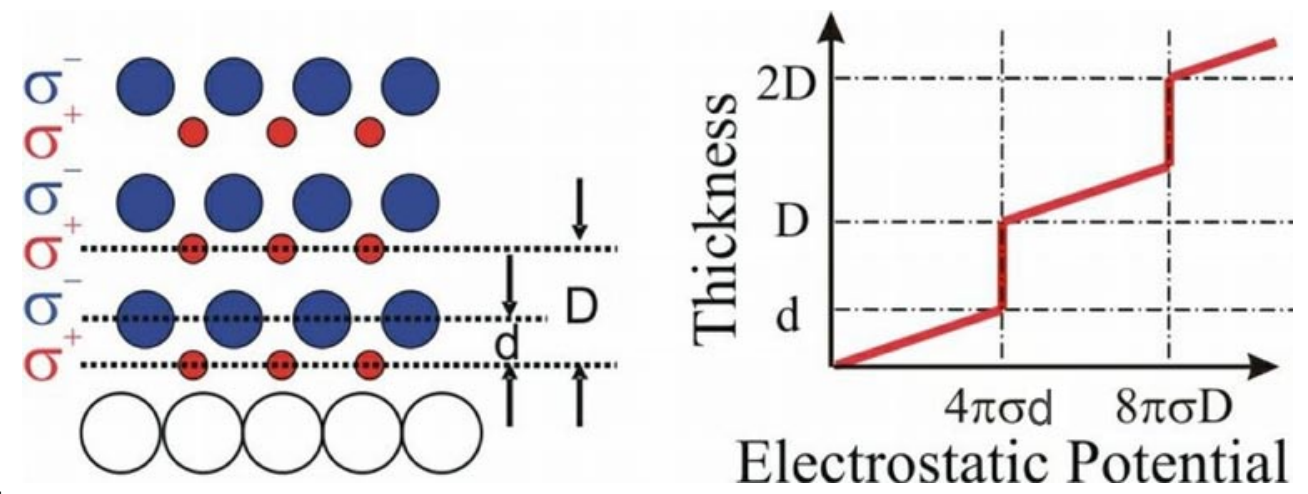

Fig. 3.21:

Alternation of positively and negatively charged oxide planes (left) leads to an electrostatic potential that gradually increases with the thickness of the polar oxide film (right). ${ }^{33}$

Polar oxide surfaces become thermodynamically stable when the divergence in the surface potential can be suppressed by removing those excess charges that lead to the uncompensated surface dipole. Several mechanisms have been identified that enable the healing of surface polarity. A first possibility is the restructuring of the top-most surface layers, creating planes of fractional atom occupancy and hence modified charge distribution. The best studied example is the octopolar reconstruction of the rocksalt (111) surface that was predicted by Wolf $^{273}$ and later verified experimentally for $\mathrm{NiO}(111)^{34}$ and $\mathrm{MgO}(111)$ surfaces. $^{274}$ The octopolar reconstruction comprises two surface layers with $75 \%$ and $25 \%$ atom filling with respect to the ideal plane, residing on top of a bulk-truncated oxide layer. The dipole compensation in this case is so effective that the free-energy of the reconstructed surface drops even below the value for comparable non-polar oxide planes. ${ }^{34}$ Alternative reconstruction schemes have been identified for other lattice structures and different chemical environments. For instance, a spinel-like termination was found to develop on $\mathrm{NiO}(111)$ at low $\mathrm{O}_{2}$ chemical potentials, ${ }^{275}$ and several non-stoichiometric surface compositions were revealed on $\mathrm{SrTiO}_{3}(111)$ as a function of the chemical environment. ${ }^{276}$

Dipole compensation might also be achieved via purely electronic effects, leaving the atomic surface structure essentially unchanged. One proposed mechanism is the creation of surface states, whose electron filling is adapted in a way that excess charges are removed. The formation of a partly filled electronic state is directly connected with the metallization of the oxide surface and was predicted to be the dominant polarity-healing mechanism for bulk $\mathrm{Al}_{2} \mathrm{O}_{3}(0001)^{277}$ and thin, unreconstructed $\mathrm{MgO}(111)$ films. ${ }^{278}$ The formation of dipolecompensating surface states becomes particularly easy when the polar oxide is capped by a thin metal film, as demonstrated for Cu over-layers on $\mathrm{ZnO}(0001) .{ }^{279,280}$ 
A third way to remove the uncompensated dipole of polar oxide surfaces is the binding of adspecies that become charged upon adsorption. The prototype adsorbate to heal surface polarity is hydrogen, which forms hydroxyl groups consisting of a positively charged $\mathrm{H}^{+}$ion and a surface oxygen atom. The hydroxylation of oxide surfaces is often triggered by the heterolytic splitting of water, which renders this compensation mechanism especially efficient for oxide surfaces in ambient environments. Hydroxylation was predicted to occur spontaneously on most rocksalt (111) surfaces ${ }^{281}$, on $\mathrm{ZnO}(0001)$ and on $\mathrm{Al}_{2} \mathrm{O}_{3}(0001) .{ }^{277}$ It has been revealed experimentally for instance for $\mathrm{MgO}(111),{ }^{282,283} \mathrm{NiO}(111)^{284,285}$ and $\mathrm{ZnO}(0001)^{286}$ by detecting the $\mathrm{O}-\mathrm{H}$ vibrational bands. Also combined mechanisms are reported; where molecular adsorption induces the formation of a partly-filled surface state at $\mathrm{E}_{\mathrm{F}}$ that in turn removes the surface polarity. ${ }^{35}$

In the case of ultrathin oxide films on metal supports, uncompensated surface polarity can be stabilized even without substantial reconstructions or attachment of charged adsorbates. The reason is an efficient screening of the unfavorable charge distribution at the oxide surface by accumulating a corresponding number of image charges in the metal underneath. ${ }^{144}$ Oxide films with polar structure might thus be prepared on metal supports, although the respective bulk material is thermodynamically instable. Well-studied examples for a support-induced stabilization of surface polarity are $\mathrm{MgO}(111)^{287,288}$ and $\mathrm{ZnO}(0001)^{289}$ on $\mathrm{Ag}(111)$, as well as $\mathrm{FeO}(111)$ on $\operatorname{Pt}(111){ }^{147,190}$

Oxide surfaces with polar termination often exhibit a different adsorption behavior and a superior chemical reactivity compared to their non-polar counterparts and a detailed understanding of polarity-driven oxide properties is therefore highly desirable. ${ }^{33}$ The STM is able to contribute to the exploration of polar oxide materials in various ways. It provides topographic images of polar surfaces, which might clarify the predominant polarity-healing mechanism of the system. Furthermore, the STM can be used to probe directly the strength of uncompensated surface dipoles, by mapping the associated changes in the surface potential. An example for this approach is given in the second section of this chapter.

The possibility to image polar oxide surfaces with the STM has first been demonstrated for $\mathrm{ZnO}(0001)$, whose polarity arises from an alternation of ionic oxygen and zinc planes along the surface normal. ${ }^{290,291}$ Triangular shaped islands and pits of $1 \mathrm{ML}$ height have been identified as the characteristic structural element of the oxide surface (Fig. 3.22). According to DFT calculations, this particular reconstruction is responsible for the suppression of the surface polarity of $\mathrm{Zn}$-terminated $\mathrm{ZnO}(0001) .{ }^{292}$ In principle, uncompensated surface charges 
introduced by the Zn top-layer can be removed by decreasing the Zn:O stoichiometry from the bulk value of $1: 1$ to $3: 4$ at the surface. Such an oxygen-rich surface is indeed realized by creating the observed triangular structures (islands and pits) and saturating all step edges with oxygen atoms. For an equal-sided, triangular island with $1 / 2 \cdot n \cdot(n+1)$ anions and $1 / 2 \cdot n \cdot(n-1)$ cations, the charge difference corresponds to the number of oxygen atoms $n$ bound to one side of the triangle. The desired 3:4 surface stoichiometry is now obtained for distinct islands sizes, e.g. for $n=7$. For larger structures, additional atoms have to be removed from the layer underneath to reach the ideal $\mathrm{Zn}: \mathrm{O}$ surface ratio. The patched Zn-terminated $\mathrm{ZnO}(0001)$ surface observed in the STM is in perfect agreement with this stabilization mechanism (Fig. 3.22).
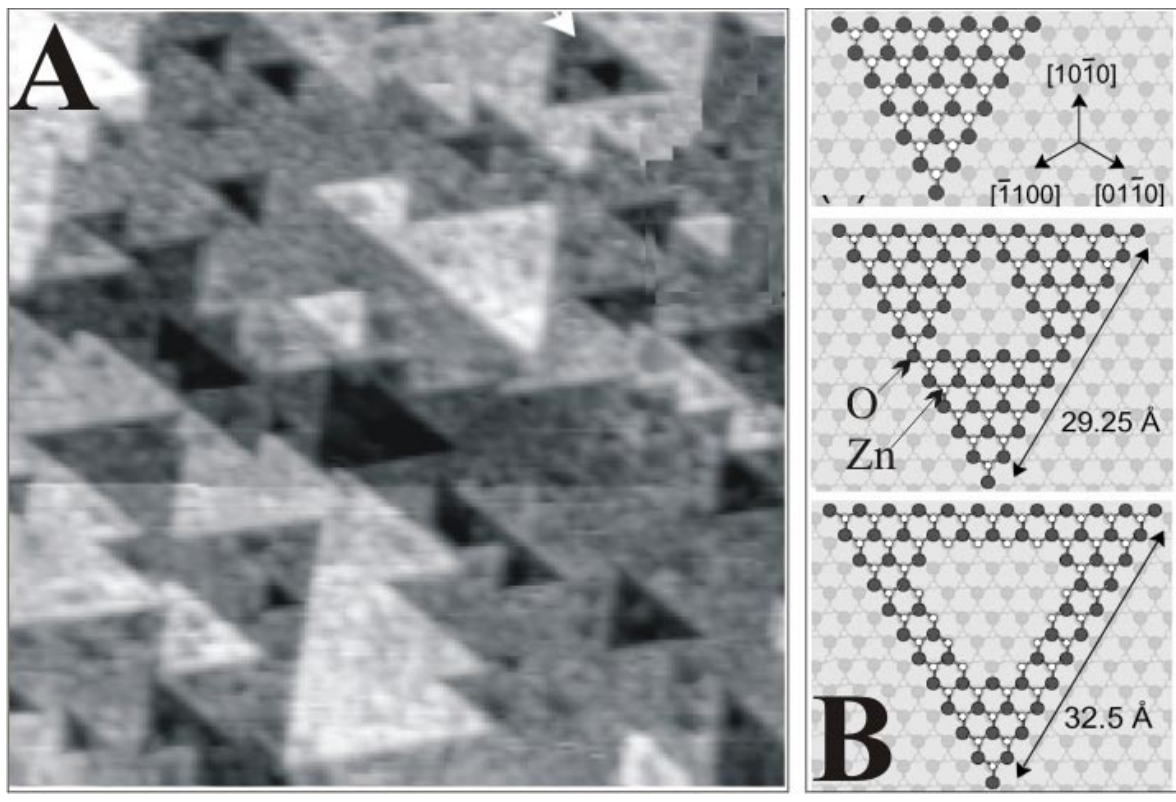

Fig. 3.22:

(A) Empty-states image of $\mathrm{ZnO}(0001)(50 \times 50$ $\mathrm{nm}^{2}$ ). The terraces are covered with triangular islands and holes, being responsible for healing the surface polarity.

(B) Structure models of different triangular islands, which are oxygen-rich with respect to the bulk stoichiometry in order to remove the surface dipole. ${ }^{291}$

In contrast, an atomically flat (0001) surface is revealed for thin $\mathrm{ZnO}$ films grown on $\mathrm{Ag}(111){ }^{289}$ The polarity is suppressed in this case, as the first two atomic layers adopt a boron-nitride structure with the Zn and O ions being co-planar. The wurzite structure of bulk $\mathrm{ZnO}$ is, however, restored in thicker films, which is accompanied by a considerable roughening of the oxide surface in order to quench the reappearing surface dipole. The issue of polarity has been addressed also for spinel-like oxide structures, such as for bulk ${ }^{293}$ and thin film $\mathrm{Fe}_{3} \mathrm{O}_{4}{ }^{28}$ as well as for $\mathrm{Cr}_{3} \mathrm{O}_{4}$ on $\operatorname{Ir}(001) .{ }^{294}$ In the latter case, polarity healing is achieved by a substantial decrease of the separation between the terminating $\mathrm{Cr}$ layer and the $\mathrm{O}$ plane below and a concomitant reduction of the ionicity of the surface species. Other polarityhealing mechanisms, such as changes of the stoichiometry and atomic structure at the surface or adsorption of charged species could be excluded with the help of STM measurements. 
A similar means to reduce uncompensated surface polarity has been identified for (111)oriented films with rocksalt structure, in particular for FeO/Pt(111). ${ }^{295}$ The FeO film has a bilayer structure with a close-packed hexagonal O layer at the surface and a hexagonal Fe plane at the interface. Due to the net-charges of the $\mathrm{Fe}^{\delta+}$ and $\mathrm{O}^{\delta-}$ ions, the film has a polar character and belongs to the type III surfaces according to Taskers scheme. ${ }^{272}$ However, the Fe-O layer separation, determined with $0.68 \AA$ by X-ray diffraction, ${ }^{172}$ is $50 \%$ smaller than in bulk FeO, which leads to a substantial decrease of the surface polarity. As a result of this vertical contraction, the FeO layer is expanded within the (111) plane and the Fe nearest-neighbor distance increases from $3.0 \AA$ in the bulk to $3.1 \AA$ in the thin film. A similar tetragonal lattice distortion has been revealed for other polar films, e.g. VO/Rh(111) ${ }^{222,296}$ and $\mathrm{TiO}_{\mathrm{x}} / \mathrm{Pt}(111){ }^{181}$ indicating the universal nature of this mechanism to lower the electrostatic energy.

In addition to the characterization of the surface morphology, the STM is capable to probe the surface potential of polar oxide films directly. Two techniques are suitable to provide information on this quantity: (i) Evaluation of the energy position of field-emission resonances in the tip-sample gap and (ii) detection of the local barrier height experienced by the tunneling electrons. Both approaches have been employed to analyze the local surface potential of polar $\mathrm{FeO}(111)$ films grown on $\mathrm{Pt}(111)$ and shall be discussed in the following. ${ }^{190}$
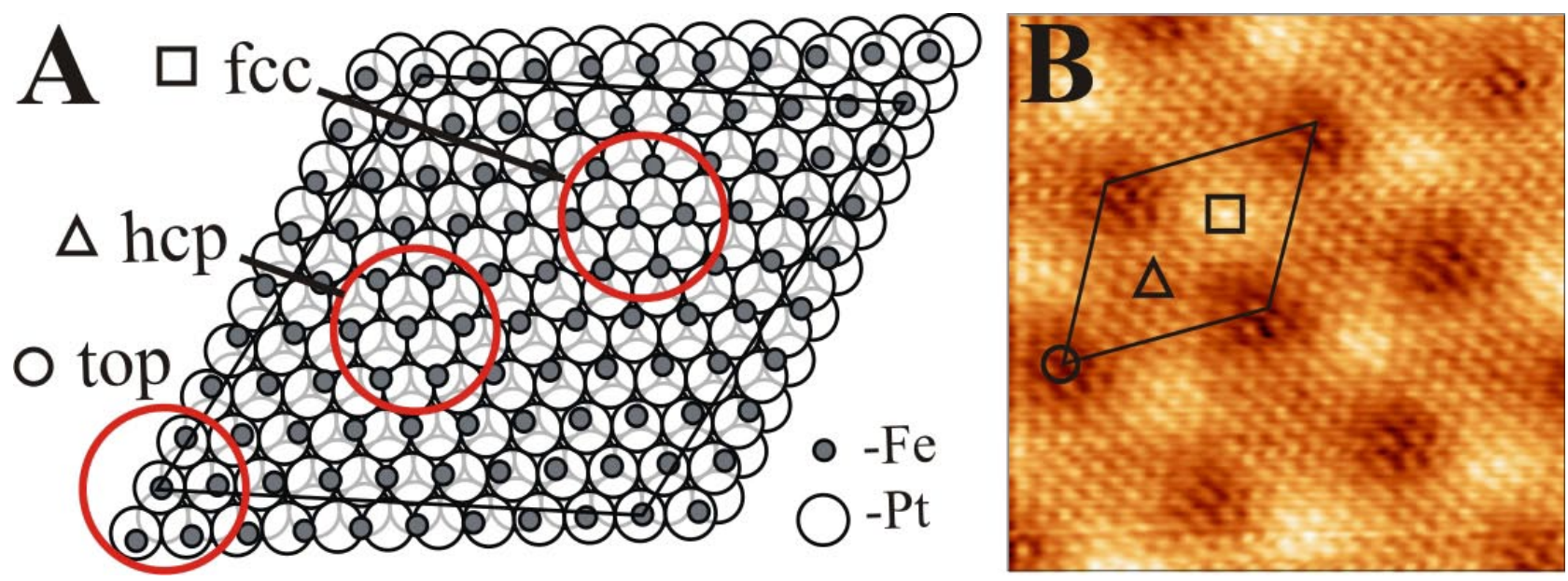

Fig. 3.23:

(A) Structure model of the coincidence cell formed between $\mathrm{FeO}$ and $\mathrm{Pt}(111)$. The O-top layer is omitted for the sake of clarity. (B) STM topographic image taken at $65 \mathrm{mV}\left(7 \times 7 \mathrm{~nm}^{2}\right)$. The different stacking domains are assigned in accordance to the DFT calculations presented in Ref. 166.

Due to a lattice mismatch of $11 \%$, the FeO bilayer forms a coincidence structure with an hexagonal unit cell of $25 \AA$ size and a crystallographic relation of $(\sqrt{ } 91 \times \sqrt{ } 91) \mathrm{R} \pm 5.2^{\circ}$ with 
respect to the underlying $\operatorname{Pt}(111)$ (lattice parameter: $2.76 \AA$ ). ${ }^{147}$ Within the coincidence cell, three stacking domains are distinguishable that differ in their Fe binding geometry on the $\mathrm{Pt}$ support (Fig. 3.23). In the top domain, Fe atoms bind on top of Pt and O atoms occupy fcc hollow sites. On the fcc (hcp) domains, Fe binds on fcc (hcp) hollow sites of the Pt(111), while $\mathrm{O}$ sits in hcp (top) lattice positions. The different binding configurations give rise to a distinct contrast in STM topographic images that has been related to the underlying domain structure by model calculations using $\mathrm{DFT}^{166}$ as well as a scattering formalism based on the Hückel theory (Fig. 3.23). ${ }^{174}$

The location of neighboring $\mathrm{Fe}$ and $\mathrm{O}$ atoms either in hollow or top positions of the $\mathrm{Pt}$ substrate results in local changes of the interlayer distance and consequently affects the surface dipole. The associated modulation of the surface polarity is an observable quantity, and gives rise to a strong bias-dependent contrast of the FeO film in STM images taken in the field-emission regime (Fig. 3.24). At $4.25 \mathrm{~V}$, one particular region of the coincidence cell (here marked with a circle) is imaged with a $2 \AA$ larger apparent height than the surrounding domains although the low-bias corrugation that reflects the true surface topography is below $0.3 \AA$ (see Fig. 3.23). The contrast enhancement at high bias voltages is therefore of electronic origin, as verified with the differential conductance maps shown in the top part of Fig. 3.24. In correspondence to the topographic images, high $\mathrm{dI} / \mathrm{dV}$ intensity is observed at $4.25 \mathrm{~V}$ in the domains marked by the circle. With increasing sample bias, the conductance maximum moves to the domains depicted by a square that turns bright at $4.5 \mathrm{~V}$. It finally localizes in the triangular regions at around $4.9 \mathrm{~V}$ sample bias. ${ }^{190}$
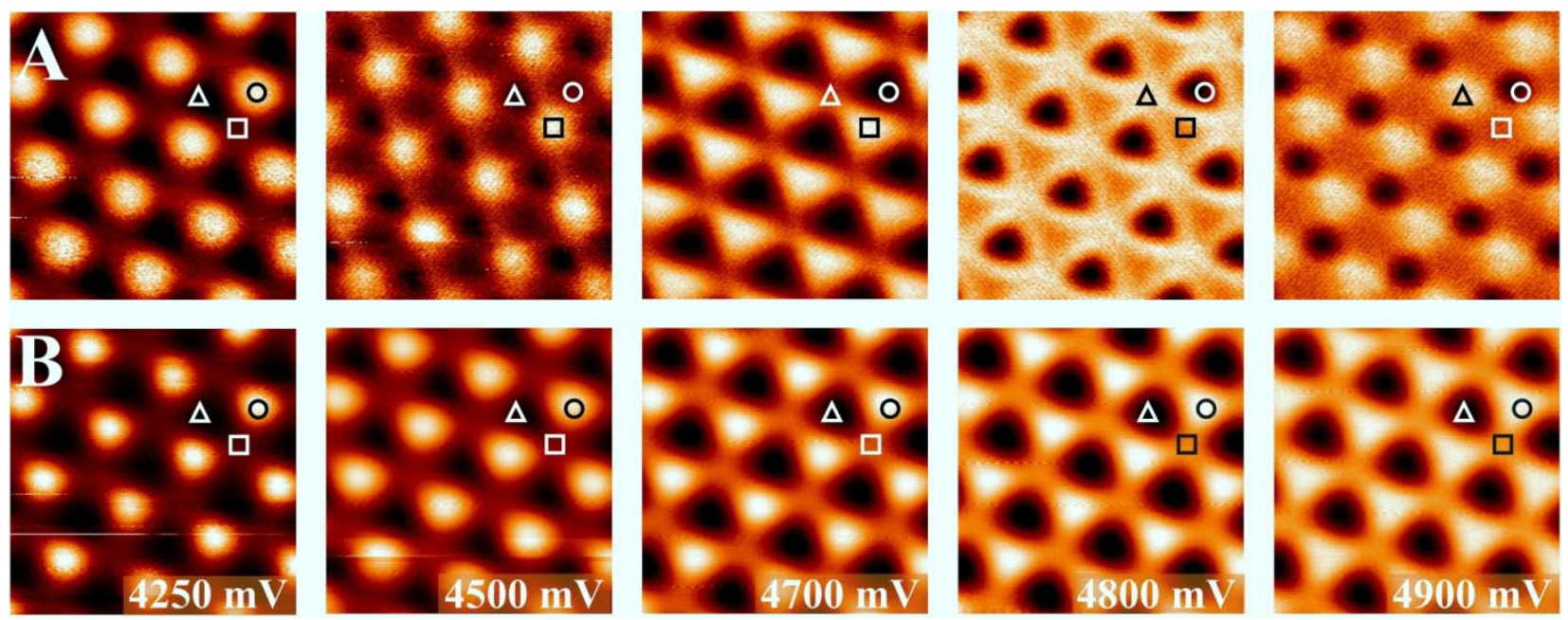

Fig. 3.24: (A) $\mathrm{d} / \mathrm{dV}$ and (B) topographic images of $\mathrm{FeO} / \mathrm{Pt}(111)$ taken as a function of the bias voltage $\left(9 \times 9 \mathrm{~nm}^{2}\right)$. The contrast change reflects the varying contributions of FER to the electron transport through the different stacking domains. 
Similar to the $\mathrm{MgO} / \mathrm{Mo}(001)$ case discussed in Chapter 3.5, these bias-dependent conductance changes are caused by the availability of field emission resonances (FER) in the tip-sample junction that are in turn sensitive to the local surface potential. FER represent extremely efficient conductance channels that are available for electron transport at bias voltages higher than the $\mathrm{FeO} / \mathrm{Pt}(111)$ work function. At this condition, that part of the STM junction that is confined by the sample surface on one side and the down-sloping vacuum barrier on the other becomes classically accessible (Fig. 3.25B). Electrons penetrating the classical region are now able to form standing waves, if multiples of half their electron-wavelength match the distance between the two boundaries, and propagating and reflected waves interfere constructively. The resulting quasi-bound electronic states, being termed field-emission resonances, are characterized by a high transmission probability and carry a large fraction of the electron current at elevated sample bias. ${ }^{74,297}$ Their energy positions depend on the local surface potential $\Phi$ in a way that increasing $\Phi$ values induce an up-shift of the resonance levels (Fig. 3.25B)

Based on this simple model, the contrast change in the dI/dV maps of FeO/Pt(111) can be translated into spatial modulations of the surface potential in the polar oxide film. The domain that turns bright at $4.25 \mathrm{~V}$ is the one with lowest $\Phi$, as the first FER becomes available for electron transport at relatively small bias values. Assuming a simple hard-sphere model, this region can be assigned to the $\mathrm{FeO}$ top domain, where $\mathrm{Fe}$ and $\mathrm{O}$ atoms occupy top and hollow sites in the Pt surface, respectively, and the Fe-O layer distance and hence the surface dipole are smallest. At $4.5 \mathrm{~V}$, the first FER is available in the next domain, which has a slightly higher surface potential and thus up-shifted resonance levels (square region). The triangular region, turning bright last in the $\mathrm{dI} / \mathrm{dV}$ maps, is therefore the one with the largest surface potential. Our hard-sphere model connects the latter region to the hcp domains, where the vertical distance between Fe atoms in Pt hollow sites and $\mathrm{O}$ atoms in on-top positions is expected to be largest. The sequential availability of FER in the different domains is not so apparent in the topographic images, which probe the integral conductance over a large bias window (Fig. 3.24B). This phenomenon can be understood by the fact that low-lying FER still carry parts of the tunnel current even if they are not in resonance with the tip Fermi level. 

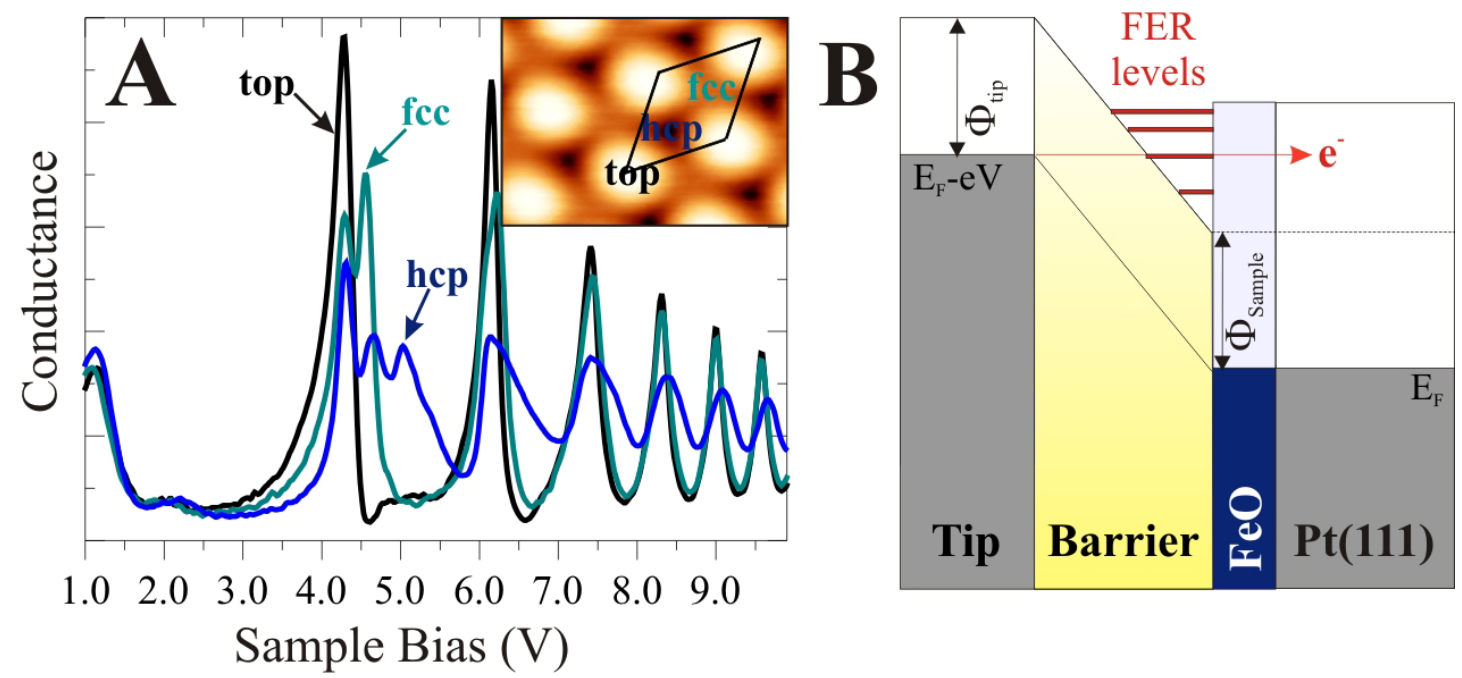

Fig. 3.25: (A) $d \mathrm{I} / d V$ spectra obtained with closed feedback loop for the three stacking regions of the $\mathrm{FeO}$ film, as indicated in the STM image shown in the inset. Each maximum marks the position of a field-emission resonance (FER) in the tip-sample junction. (B) Potential diagram visualizing the development of FER at high bias voltage and their dependence on the surface potential.

The variation in the local surface potential can even be quantified for the three stacking domains of the $\mathrm{FeO} / \mathrm{Pt}(111)$ film. ${ }^{190}$ For this purpose, the FER energy-positions are determined in the different regions of the coincidence cell via $\mathrm{dI} / \mathrm{dV}$ spectroscopy performed in a large bias window (1-10V) (Fig. 3.25A). In accordance to the $\mathrm{dI} / \mathrm{dV}$ maps, the lowest resonance state occurs in the top domain at $4.25 \mathrm{~V}$ (marked by the circle), whereas the fcc (square) and hcp (triangle) domains follow at 4.7 and $5.1 \mathrm{~V}$, respectively. The higher-order FER show the same sequence in the three domains, although the energy separation between the $\mathrm{dI} / \mathrm{dV}$ maxima gradually decreases. It should be mentioned at this point that due to the limited spatial resolution of $\mathrm{dI} / \mathrm{dV}$ spectroscopy at high bias, low-lying FER in neighboring domains contribute to the spectral response. As a consequence, the dI/dV peaks in fcc and hcp domains are split in two and three maxima, respectively, with the lower ones reflecting the conductance contributions from the adjacent top and fcc domains.

The surface potential $\Phi$ can now be determined by fitting the experimental FER positions to a simple model, which treats the resonances as the eigen-states of a triangular potential. The well is confined by the sample surface and the vacuum barrier, sloping down in accordance to the tip-induced electric field (Fig. 3.25B). ${ }^{269}$ The surface potential is explicitly included in the approach, as it sets the bottom of the potential and provides an offset to all FER levels. Assuming a constant tip-electric field $F$, being adjusted by the enabled feed-back loop during spectroscopy, the position of the $n^{\text {th }}$ FER calculates to: $e V_{n}=\Phi+\left(\frac{3 \pi \hbar e}{2 \sqrt{2 m}}\right)^{2 / 3} F^{2 / 3} n^{2 / 3}$, whereby 
$m$ is the free electron mass. ${ }^{269}$ The $\Phi$ value for all three domains is now deduced from fitting the experimental FER positions to the calculated ones. The first level is hereby excluded, because its energy is strongly altered by image potential interactions that are not considered in the model. ${ }^{269}$ In good agreement with the results of the $\mathrm{dI} / \mathrm{dV}$ maps, the surface potential rises when going from the top domain $(3.5 \mathrm{eV})$ to the fcc $(3.65 \mathrm{eV})$ and hcp domain $(3.85 \mathrm{eV})$, reflecting the increase of the interlayer distance predicted by the hard sphere model.
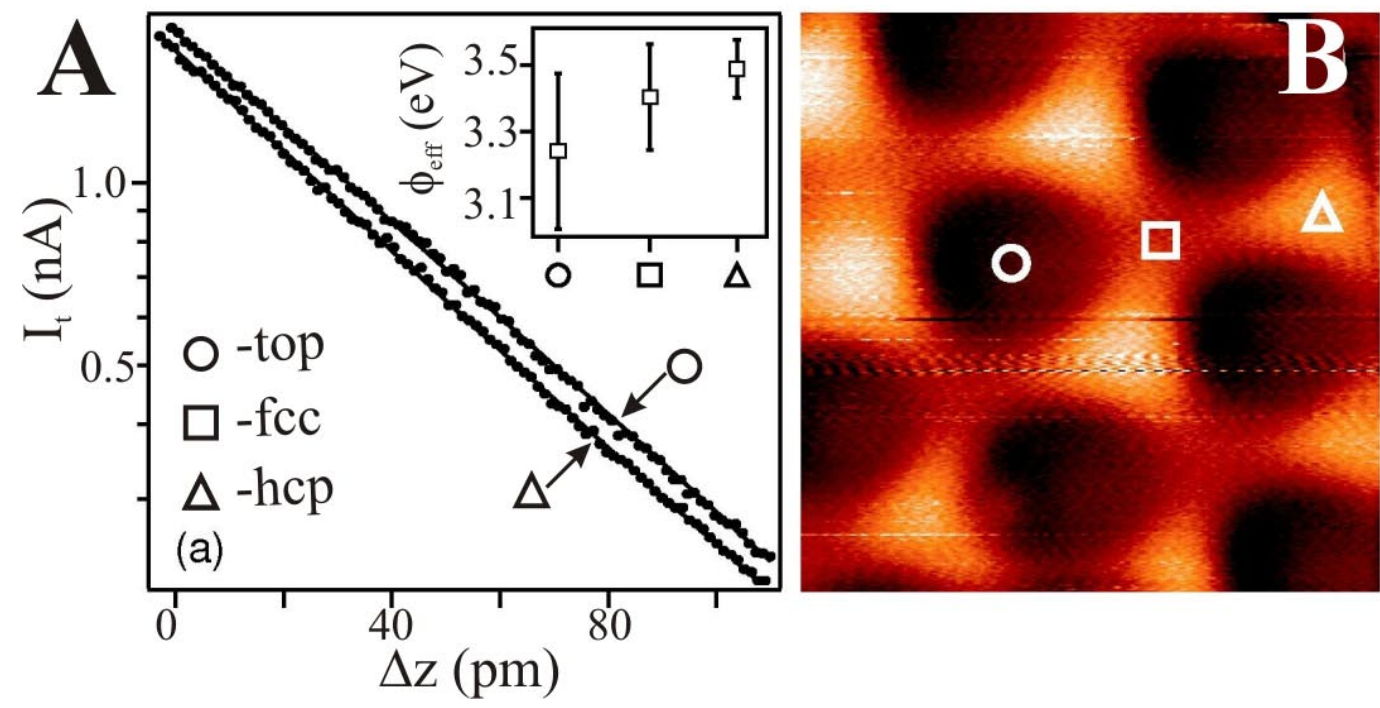

Fig. 3.26: (A) Tunnel current versus tip-sample separation recorded for the top and hcp domain of the $\mathrm{FeO}$ coincidence cell. From the current slope, the effective barrier height is determined, as shown in the inset. (B) $d(\operatorname{lnI}) / d z$ image of $\mathrm{FeO} / \mathrm{Pt}(111)$ taken with lock-in technique and closed feed-back loop (Image size $5.7 \times 5.7 \mathrm{~nm}^{2}, V_{s}=4.5 \mathrm{~V}, \Delta z_{\mathrm{rms}}=1 \AA$ ).

Compatible information on the local surface potential is obtained from spatial variations of the effective barrier-height $\Phi_{\text {eff }}$ that is experienced by electrons tunneling from the tip to the sample. This quantity is experimentally determined from evaluating the distance dependence of the tunnel current $I$ in an STM junction ( $d I / \mathrm{dz}$ spectroscopy). ${ }^{59}$ The underlying interrelation can be easily derived from a simple one-dimensional model for electron tunneling through a square-shaped barrier and holds for small bias voltages only: $\Phi_{\text {eff }}=\frac{\hbar^{2}}{8 m}\left(\frac{d \ln I}{d z}\right)^{2} \cdot{ }^{60}$ It should be emphasized that the effective barrier height is not identical to the real surface potential, because the effects of the tip work function, of image potential interactions and realistic barrier shapes are not considered. ${ }^{298}$ The barrier height is, nonetheless, a homogenous function of the surface potential $\Phi$, and consequently reproduces the correct order of $\Phi$ within the different FeO stacking domains. Figure 3.26 shows two approaches to determine the effective barrier height in an STM junction. In (A), the logarithmic current response to a linear distance 
ramp is recorded and $\Phi_{\text {eff }}$ is extracted from the slope of the $\ln (I)$-z curves. In (B), the $d(\ln I) / d z$ signal is directly mapped with lock-in technique by superimposing a small distance modulation to the feed-back response during scanning. In both cases, spatial variations of the effective barrier height are detected on the FeO surface. The slope of the $\ln (I)$ - $z$ curves is found to be smallest in the top-domain, indicating the lowest barrier height in this region. Accordingly, the same oxide domain turns dark in the $d(\operatorname{lnI}) / d z$ maps. The opposite behavior is found for the hcp-domain, reflecting the higher $\Phi_{\text {eff }}$ value in this stacking region. Both analyses, based either on measuring the FER energies or the effective barrier height, therefore reveal similar spatial variations of the local surface potential. As those $\Phi$ modulations are directly linked to the local $\mathrm{Fe}^{\delta+}-\mathrm{O}^{\delta-}$ layer distance, they provide a measure for the surface polarity in the three FeO stacking domains.

In the experiments presented so far, the assignment of the different oxide regions to top, fcc and hcp stacking domains is exclusively based on a simple hard-sphere model for estimating the interlayer distances. To link the polarity modulations observed in the STM to the true atomic structure of the $\mathrm{FeO} / \mathrm{Pt}(111)$ film, complementary theoretical investigations are required. The FeO film poses, however, a big challenge to any theoretical investigation. First, the large size of the coincidence cell formed between FeO and Pt support is not treatable with reasonable computational effort and simplified model geometries have to be used instead. In particular, three pseudomorphic FeO-Pt systems with a single lattice constant of $3.1 \AA$ were constructed, placing the Fe atoms either in the top, hcp or fcc positions of the $\operatorname{Pt}(111)$ surface. ${ }^{168}$ Alternatively, different FeO-Pt superstructures have been used that are much smaller than the original coincidence cell but contain Fe atoms in all characteristic binding configurations (Fig. 3.27A,B). ${ }^{166}$ Second, transition metal oxides are difficult to treat with conventional DFT in general due to the high degree of electron correlation in their spatially confined $d$-levels. ${ }^{18}$ The calculations were therefore performed at the DFT+U level, using the generalized gradient approximation (GGA) and the Perdew-Wang 91 functional. ${ }^{299,300}$ And third, a variety of ferromagnetic and anti-ferromagnetic Fe spin configurations needs to be tested for the FeO bilayer in order to identify the one with lowest total energy (Fig. 3.27C). ${ }^{301}$ For comparison, bulk FeO (wüstite) has an anti-ferromagnetic order, arising from the parallel alignment of $\mathrm{Fe}$ magnetic moments in the (111) planes and an anti-parallel stacking of adjacent planes. 


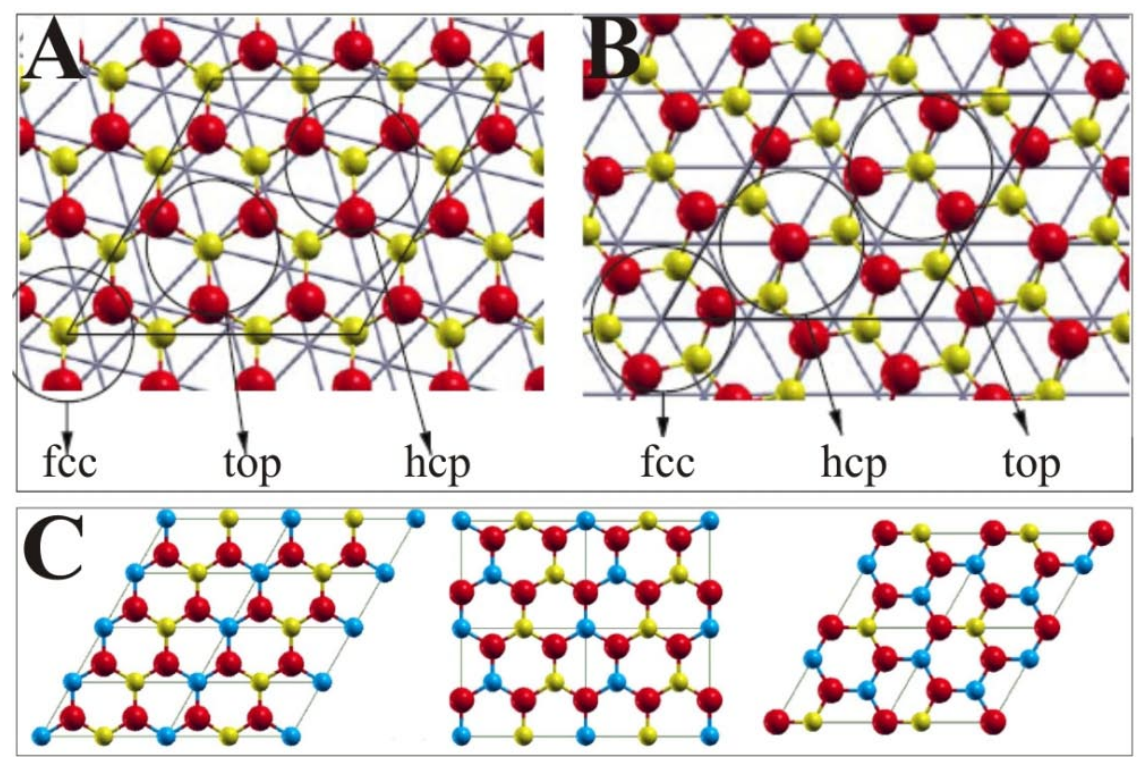

Fig. 3.27: Top view of two FeO/Pt(111) model structures formed by (A) the superposition of a $(3 \times 3)$ $\mathrm{FeO}$ and a $(\sqrt{13} \times \sqrt{ } 13) \mathrm{R} 14^{\circ} \mathrm{Pt}$ lattice and by $\mathbf{( B )}$ a $(\sqrt{ } 7 \times \sqrt{ } 7) \mathrm{R} 19^{\circ} \mathrm{FeO}$ combined with a $(3 \times 3) \mathrm{Pt}(111)$ unit cell. (C) Anti-ferromagnetic spin configurations tested for the FeO thin film: row-wise (left), zigzag (middle) and $(\sqrt{ } 3 \times \sqrt{3}) R 30^{\circ}$ spin alignment (right). The latter one can be considered as the collinear analog of the $120^{\circ}$-Néel structure. Large red spheres depict the $\mathrm{O}$ atoms, small yellow and blue ones are Fe atoms with opposite spin orientation. ${ }^{166}$

In all model structures, the Fe atoms have a constant magnetic moment of $3.6 \mu_{\text {Bohr. }}{ }^{166}$ The ferromagnetic spin ordering is generally found to be higher in energy than anti-ferromagnetic ones, whereby the $120^{\circ}$ Néel configuration is energetically preferred out of the limited set of tested spin structures (Fig. 3.27). This finding is in agreement with paramagnetic resonance spectroscopy, which did not reveal a ferromagnetic spin signal from the FeO film. ${ }^{301}$ In both, the pseudomorphic and the non-pseudomorphic computational cells, the oxide regions with Fe in hcp and fcc binding sites have rather similar properties independent of the assumed magnetic structure, whereas the top domain sticks out in various aspects. It is characterized by the largest interfacial separation from the Pt(111) surface and therefore mimics the properties of a free standing FeO layer. Additionally, its vertical separation between the Fe and O planes is $15-20 \%$ smaller than in the hcp and fcc domains, rendering the $\mathrm{Fe}^{\delta+}-\mathrm{O}^{\delta-}$ surface dipole and hence the surface potential smallest in this region. In contrast, the hcp and fcc domains possess pronounced surface polarity due to the larger Fe-O layer separation. With respect to the cell average, the computed surface potential is $0.15 \mathrm{eV}$ lower in the top domains, but 0.08 $\mathrm{eV}$ and $0.14 \mathrm{eV}$ higher in the fcc and hcp regions, respectively. The total magnitude of the potential modulations across the coincidence cell is with $0.29 \mathrm{eV}$ in good agreement with the experimental value of $0.35 \mathrm{eV} \cdot{ }^{190}$ Apparently, already the simplified model structures used in 
the calculations are capable to describe the polarity modulations in the $\mathrm{FeO} / \mathrm{Pt}(111)$ film with good accuracy.

Assuming that the pronounced contrast in STM images taken at elevated sample bias is indeed governed by modulations of the surface potential (Fig. 3.24), the experimental distribution of bright and dark areas should follow the calculated surface potential within the coincidence cell. A comparison between STM topographic images and simulated surface potential maps is presented in Fig. 3.28. Hereby, the potential is shown with reversed contrast (small $\Phi$ - bright color), because a smaller $\Phi$ value enables effective electron transport through a low-lying FER in the experiment. Indeed, the oxide domain with brightest contrast in the STM image corresponds to the one with the smallest surface potential in the DFT, verifying our initial assignment of the different stacking domains in the $\mathrm{FeO}$ coincidence cell.
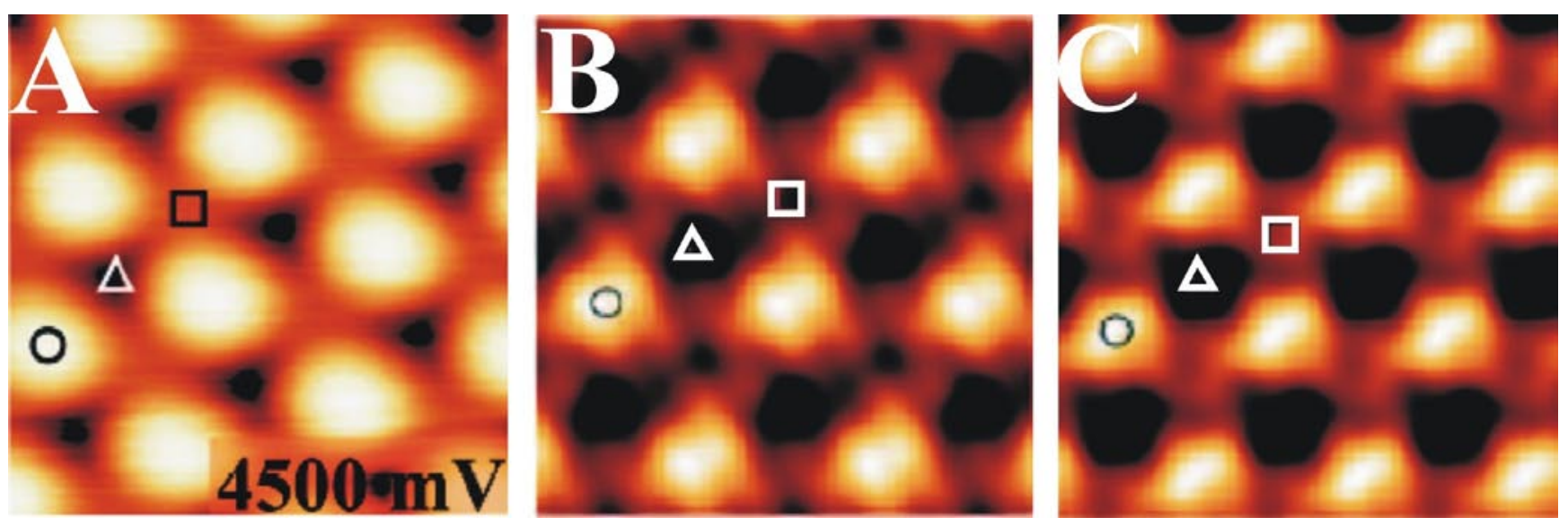

Fig. 3.28: (A) STM image of FeO/Pt(111) taken at $+4.5 \mathrm{~V}$ sample bias $\left(7.5 \times 7.5 \mathrm{~nm}^{2}\right)$. (B,C) Surface potential maps of $\mathrm{FeO} / \mathrm{Pt}(111)$ calculated for the two unit cells shown in Fig. 3.27. The maps depict the $-\Phi$ signal, and low potential values therefore appear bright. The symbols represent the three $\mathrm{FeO} / \mathrm{Pt}$ stacking domains (circle: Fe-top, triangle: hcp and square: fcc region). ${ }^{166}$

In should be noted that the assignment of the three stacking domains to the experimental data is not beyond dispute. Although other groups agree with the deviating properties of the top domain, being the one with the smallest layer distance and surface polarity, they propose a swapping of the fcc and hcp regions with respect to the model presented here. ${ }^{302,303}$ Independent of the true assignment, the experiments on the $\mathrm{FeO} / \mathrm{Pt}(111)$ system demonstrate the capabilities of STM to provide spatially-resolved information on the electrostatic potential across a polar oxide surface. The STM is therefore not only suited to characterize the various polarity-healing mechanisms from the topographic point of view, but additionally enables a determination of surface potential modulations induced by uncompensated oxide polarity. The technique does not provide quantitative values for surface potential and work function, in 
contrast to methods like Kelvin-Probe spectroscopy. However, relative changes of this quantity can be measured with good accuracy and unmatched spatial resolution.

The unusual adsorption behavior of polar oxide surfaces and the contribution of STM to their exploration will be in the focus of Chapter $\underline{4.4 .2}$.

\section{Adsorption on oxide surfaces}

After discussing the properties of pristine oxide surfaces and introducing experimental approaches for their investigation with STM, the second part of this review concentrates on the adsorption characteristics of oxide materials. Thanks to the immense progress in the understanding of adsorption mechanisms on bulk oxides, this topic will be presented first, while deviations from those general principles in thin-film oxides are presented thereafter. The adsorption behavior of oxide films on metal supports can be divided into two scenarios. For a film thickness that is large enough to decouple the topmost oxide layer from the metal below, comparable adsorption properties are expected for thin-film and bulk oxides. In this case, the thin-film approach is suitable to model the respective bulk system, however, ensures electrical conductivity and provides maximum control over the stoichiometry, polarity and defect density in the oxide surface. In the limit of ultra-thin films, on the other hand, new adsorption mechanisms emerge that arise from the contribution of the metal support to the oxide binding behavior. Hereby, long-ranged dispersive and electrostatic interactions between adsorbate and support are of special importance, because the influence of the metal underneath prevails even for thicker oxide films. The applicability of an oxide film as model system for the respective bulk material therefore depends not only on the film thickness, but also on the nature of the oxide-adsorbate interaction.

An ultrathin oxide film might be a versatile and interesting support on its own even if its adsorption characteristic is still modified by the metal underneath. In a metal-oxide compound system, several structural and electronic parameters can be tailored in a controlled way in order to tune the adsorption behavior. The binding strength of an adsorbate to the oxide surface become therefore an adjustable quantity, for example by modifying the chemical and crystallographic nature of the metal below, by inserting doping centers and defects, and by varying the film thickness. A similar flexibility is usually not achievable for bulk oxides, which renders thin oxide films a promising approach to create tunable adsorption systems 
with potential applications in catalysis, micro-electronics and optics. ${ }^{53}$ The next chapter presents a short introduction into the theory of the different adsorption mechanisms on oxide surfaces, while experimental aspects are discussed in Chapter $\underline{4.2}$.

\subsection{Adsorption on bulk oxides - Theory}

\subsubsection{Stoichiometric surfaces}

Adsorption on defect-free oxides is generally weak due to the high degree of bond saturation of their surface atoms. ${ }^{18,19,36}$ Especially oxygen-terminated surfaces are chemically inert, and physisorption, viz. van-der-Waals and polarization-interactions, are the dominant adsorption mechanisms. The binding becomes even weaker when the oxide material exhibits a band gap around the Fermi level, and no electronic states are available for hybridization with the energy levels of the adsorbate. According to DFT calculations, ${ }^{304,305,306}$ TDS $^{307,308,309}$ and microcalorimetry studies, ${ }^{310}$ the binding energy of metal adatoms to stoichiometric oxide surfaces is of the order of $1 \mathrm{eV}$ but drops below $100 \mathrm{meV}$ for most closed-shell molecules (probably with the exception of water). ${ }^{311}$

The preferential interaction mechanism between metallic adsorbates and highly-ionic oxide materials, such as $\mathrm{Al}_{2} \mathrm{O}_{3}$ and $\mathrm{MgO}$, is atom polarization in the oxide Madelung field (Fig. 4.1). The resulting electrostatic attraction is usually higher at anionic than cationic surface sites, and oxygen ions are therefore the preferred binding sites for most metal adatoms. ${ }^{312,313}$ The binding strength increases with the polarizability of the adsorbate, which explains the higher adsorption energies of larger atoms (e.g. Au) with respect to smaller, less polarizable ones, such as $\mathrm{Cu}$ and $\mathrm{Ag} .{ }^{312,314}$ This effect is partly compensated by the reduced Pauli repulsion experienced by smaller ad-species, which allows them to further approach the oxide surface. Polarization in the oxide Madelung field is the predominant interaction mechanism for noble and semi-noble metal atoms on ionic oxide surfaces and leads to binding energies ranging between $0.3 \mathrm{eV}$ (e.g. Ag on $\mathrm{MgO})^{315}$ and $0.9 \mathrm{eV}\left(\mathrm{Pd}\right.$ and $\mathrm{Au}$ on $\mathrm{MgO}$ or $\mathrm{Al}_{2} \mathrm{O}_{3}$ ). ${ }^{312,316}$

Transition metal atoms, on the other hand, are able to form weak covalent bonds with the oxide anions, which leads to a substantial increase of the adsorption energy with respect to pure polarization interactions. ${ }^{312}$ Mainly the metal $d$-states and the $O 2 p$ orbitals participate in this hybridization process, which is usually not accompanied by a charge exchange. ${ }^{317}$ The binding energies, as determined with DFT, slightly increase when going from the left to the right of the periodic table of elements due to the gradually filling of the metal $d$-states. ${ }^{315,318}$ 
This trend is only interrupted for the noble metals, whose $d$-states are completely filled and too low in energy to participate in the bond formation. Transition metal atoms, e.g. $\mathrm{Cr}$, Ni, Mo, $\mathrm{W}$ and Pt atoms, are able to bind with 0.6 to $1.7 \mathrm{eV}$ to defect-free ionic oxides, whereby the exact binding strength depends on the chemical nature of oxide material and adsorbate, but also on details of the computational approach. ${ }^{312}$

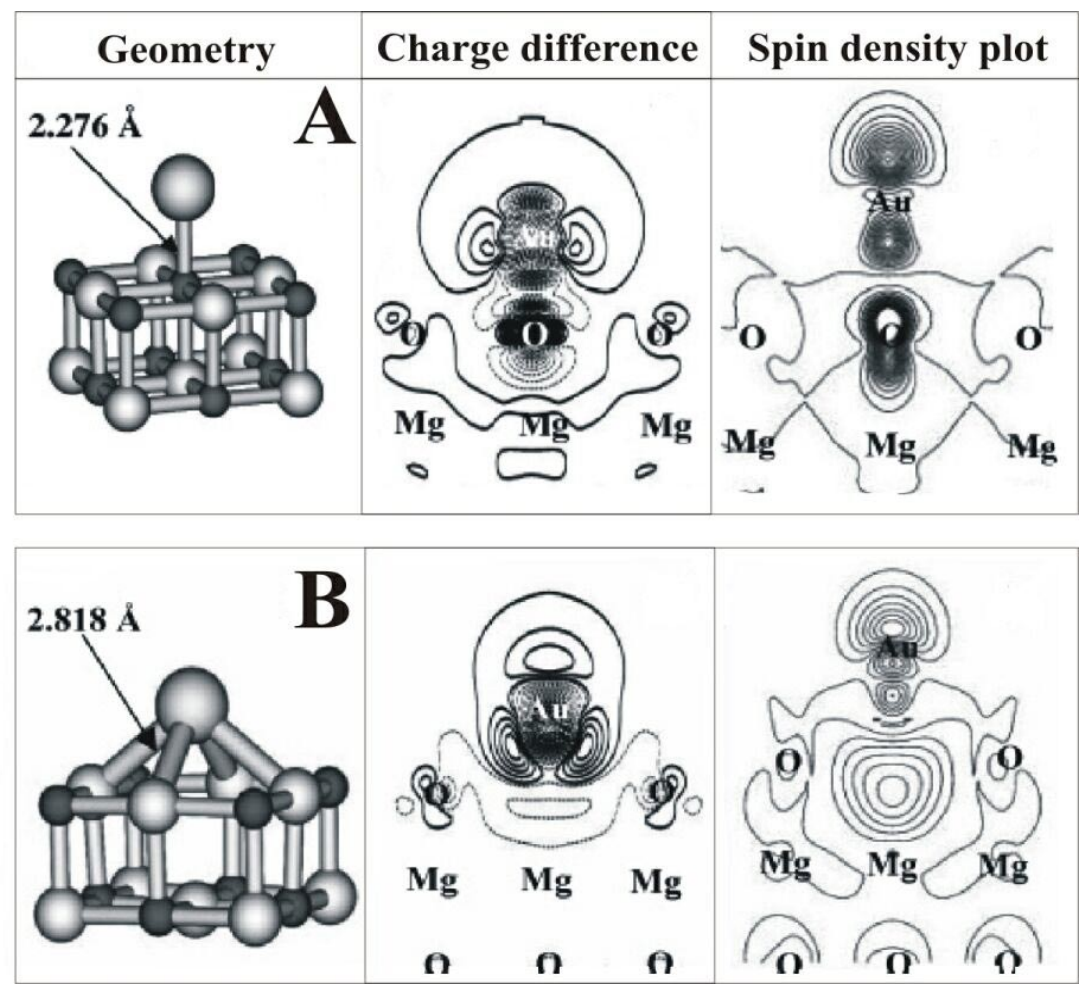

Fig. 4.1:

DFT calculation for an Au adatom bound to (A) a regular O site and (B) a doubly-occupied oxygen vacancy $\left(\mathrm{F}^{0}\right.$ center) in $\mathrm{MgO}(001)$. On the regular site, the binding is weak $\left(\mathrm{E}_{\mathrm{B}}=0.89 \mathrm{eV}\right)$ and involves mainly a redistribution of the Au charge away from the oxide anion (polarization interaction). The spin density remains localized at the Au position, indicating that the $A u$ ss atomic orbital is singly occupied as in the gas phase. On the defect site, binding is associated with an electron transfer into the Au, as deduced from the increased charge density at the adatom $\left(E_{B}=2.3 \mathrm{eV}\right)$. The resulting double occupancy of the $A u$ ss orbital is responsible for the suppressed spin density at the Au. ${ }^{316}$

The preferred adsorption site for molecules on ideal oxide surfaces depends on their donor/acceptor-type character. As a surface oxygen atom in ionic oxides behaves as a Lewis acid, it mainly attracts donor-type molecules. The most prominent reaction of this type is the formation of $\mathrm{OH}$-groups by attaching an hydrogen to a surface $\mathrm{O}^{2-}$ species, ${ }^{19,32}$ or the interaction of water with oxygen ions in the MgO surface. ${ }^{319}$ Oxide cations, on the other hand, are Lewis bases in this picture and preferentially interact with acceptor-type molecules. ${ }^{320}$ For example, CO was found to interact weakly with the $\mathrm{Mg}$ ions of $\mathrm{MgO}(001)$ with the $\mathrm{C}$ atom pointing downwards. ${ }^{321}$ 
For oxide materials with a more covalent character, polarization interactions become less important and the adsorption involves the formation of a true chemical bond (chemisorption). Also charge transfer processes between the adsorbate and the oxide support play a larger role. Binding energies of metal adatoms to covalently-bound oxides are thus considerably larger than to ionic materials. For example, $\mathrm{Cu}, \mathrm{Ag}$ and $\mathrm{Au}$ atoms bind with more than $2 \mathrm{eV}$ to stoichiometric ${ }^{313}$ and oxygen-rich $\mathrm{TiO}_{2}$ surfaces, ${ }^{322}$ as demonstrated by recent DFT calculations. On $\mathrm{V}_{2} \mathrm{O}_{3}(0001)$, the Au binding energy even reaches $2.5 \mathrm{eV}$ for the vanadium terminated surface, but drops below $1.5 \mathrm{eV}$ when the surface is covered with chemically inert vanadyl groups. ${ }^{262}$ The latter example emphasizes the decisive role of the surface termination in determining the oxide adsorption behavior.

\subsubsection{Defective surfaces}

Real oxides offer a much larger variety of binding sites due to the presence of defects in their surface, such as point (oxygen vacancies) and line defects (steps, dislocations) as well as defect clusters. The adsorption on a defective surface follows different mechanisms and typically results in binding energies that are higher than on the ideal one. Not surprisingly, IRAS and TDS experiments demonstrated that small inorganic molecules almost exclusively bind to oxide defects, while regular surface sites are only populated at high dosages and temperatures well below $100 \mathrm{~K} \cdot{ }^{36,308,323}$ Also the heterogeneous nucleation of metals on various oxide surfaces, as concluded from STM studies, provides clear evidence for the dominance of defects for the oxide adsorption charactersistic. ${ }^{37,48,251,252}$ Theoretical studies have identified different origins for the enhanced binding potential of oxide defects. ${ }^{21,317,324,325,326}$ Defects in ionic materials often contain weakly-bound excess charges that occupy localized states in the band gap and are available for the interaction with adsorbates. Positive and neutral oxygen vacancies $\left(\mathrm{F}^{+}\right.$and $\mathrm{F}^{0}$ centers), for instance, trap one and two electrons at the defect site, respectively. The associated gap states are able to hybridize with the energy levels of the adsorbate, whereby a true chemical bond is formed and electrons are transferred into the ad-species (Fig. 4.1). ${ }^{316}$ The strength of this interaction depends on the energy/symmetry match between the defect states and the adsorbate affinity levels as well as on the number and spatial localization of the trapped electrons. Consequently, two-fold positively charged $\mathrm{F}^{2+}$ centers without extra electrons have a lower binding potential than their $\mathrm{F}^{0}$ and $\mathrm{F}^{+}$counterparts. In many cases, the $\mathrm{F}^{+}$vacancy turns out to 
be the most reactive one due to the radical character of its unpaired electron. ${ }^{316,318,326}$ Similar considerations as for oxygen vacancies also hold for low-coordinated step and corner atoms in the oxide surface, which offer an equally attractive binding environment for adsorbates. ${ }^{23,243}$
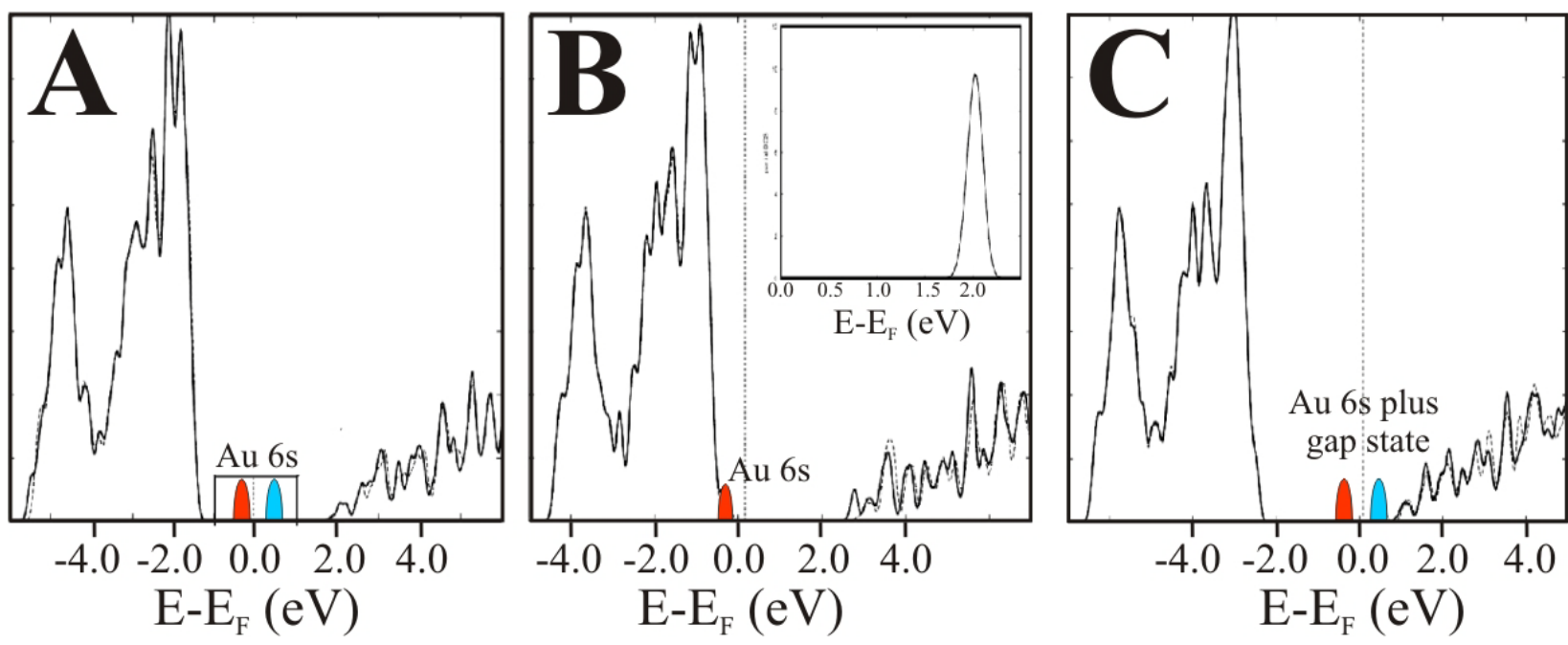

Fig. 4.2: Electronic structure of an Au adatom adsorbed on (A) a regular $\mathrm{MgO}$ anion, (B) a doublyoccupied $\left(\mathrm{F}^{0}\right.$ center) and $(\mathbf{C})$ a singly-occupied $\mathrm{O}$ vacancy $\left(\mathrm{F}^{+}\right.$center). In $(\mathrm{A})$, the adatom interacts only weakly with the oxide and the occupied $\alpha$ (red) and unoccupied $\beta$ component (blue) of its $A u 6 s$ orbital remain close to its initial position at the Fermi level. In (B), the $A u$ 6s becomes filled with an electron from the $\mathrm{F}^{0}$ center and shifts below $\mathrm{E}_{\mathrm{F}}$ (red peak). The inset shows the state density of the defect prior to $\mathrm{Au}$ adsorption. On the $\mathrm{F}^{+}$center $(\mathrm{C})$, strong hybridization between the defect state and the $A u 6 s$ orbital takes place, resulting in two hybrid states located above and below the Fermi level. ${ }^{316}$

Naturally, the character of a bond to an oxide defect depends on the chemical identity of the adsorbate as well. Transition metal atoms, such as $\mathrm{Cr}, \mathrm{Mo}, \mathrm{W}$ and Ni, interact primarily via their partly filled $d$-states at the Fermi level, which strongly overlap with the defect states in the oxide band gap. ${ }^{318}$ In noble and near-noble metals, on the other hand, s,p-orbitals are more important, as the $d$-states are completely filled and relatively low in energy. ${ }^{316,326}$ In both cases, occupied as well as unoccupied orbitals around $\mathrm{E}_{\mathrm{F}}$ are required in the adsorbate to enable hybridization and charge exchange with the oxide defect states. Figure 4.2 demonstrates a typical example for a defect-mediated adsorption scheme, which is the electronic interaction of a single Au atom with different $\mathrm{MgO}$ color centers and the ideal surface. For both, the $\mathrm{F}^{0}$ and the $\mathrm{F}^{+}$vacancy, the half-filled 6 s orbital of gas-phase Au takes a second electron due to charge transfer from the defect. While in the $\mathrm{F}^{0}$ case, the filled Au $6 \mathrm{~s}$ orbital shifts down to the MgO valence band onset $\left(\mathrm{E}_{\mathrm{B}}=3.17 \mathrm{eV}\right)$, it strongly hybridizes with the defect states of the $\mathrm{F}^{+}$centre and splits into an Au-like component below and a defectrelated component above the Fermi level $\left(\mathrm{E}_{\mathrm{B}}=3.97 \mathrm{eV}\right) .{ }^{316}$ Similarly, binding of a Pd atom to $\mathrm{F}^{0}$ and $\mathrm{F}^{+}$color centers is associated with the transfer of one electron into the $P d 5 \mathrm{~s}$ orbital, whereas no charge exchange takes place on the regular MgO surface. ${ }^{317,326,327}$ Due to the 
unoccupied nature of the $P d 5 s$ in the gas-phase atom, the sequence of adsorption energies is reversed and the doubly occupied $F^{0}$ centre binds the Pd atom stronger $\left(E_{B}=3.42 \mathrm{eV}\right)$ than the $\mathrm{F}^{+}$vacancy $\left(\mathrm{E}_{\mathrm{B}}=2.10 \mathrm{eV}\right)$. In general, the binding energy of metal atoms to ionic oxides is roughly two to four times larger on defects than on regular surface sites, explaining the decisive importance of oxide defects for the nucleation and growth of metals.

In $\mathrm{SiO}_{2}$, as an example for a non-reducible oxide with strong covalent character, the removal of an oxygen atom results in the formation of either a new bond between the two $\mathrm{Si}^{3+}$ neighbors $\left(\mathrm{V}_{0}\right.$ center) or of two Si dangling bonds ( $\mathrm{E}$ ' centers). ${ }^{325,328}$ Especially the latter defect type offers an attractive binding environment for adsorbates. The adsorption is mediated in this case by covalent interactions between the dangling-bond state of the Si that contains a single unpaired electron and an adsorbate orbital. It does usually not involve a charge transfer into or out of the ad-species. The resulting binding energies can be substantial, reaching $3.52 \mathrm{eV}$ for an $\mathrm{Au}$ atom attached to a $\mathrm{SiO}_{2}$-E' center for example. ${ }^{328} \mathrm{In}$ less ionic and more reducible oxides, the excess electrons left behind upon oxygen removal are not confined to the defect site but attach to neighboring cations and reduce their oxidation state. ${ }^{240,329}$ The extra electron in the surface cation is now available to fill a new hybrid state formed with the adsorbate, in analogy to the silica case. Also for oxides with strong covalent character, the binding energy to a defect site can be two times larger than to a regular surface site. However defects are less dominant for the adsorption behavior, because the overall binding energies are higher than for ionic oxides.

It should be mentioned at this point that defects are not only important for the stabilization of adsorbates, but also govern the chemical activity of oxide surfaces. Various elementary steps of a chemical reaction, for instance the activation or dissociation of a molecule, require the distinct structural and electronic environment of an oxide defect. This has been analyzed in detail for the $\mathrm{TiO}_{2}(110)$ surface, especially for the technologically important case of the water

dissociation. ${ }^{40,257,330,331}$ Studying the defect-mediated adsorption characteristic is therefore a fundamental precondition for the understanding of the catalytic activity of oxide materials.

\subsection{Adsorption on bulk oxides - Experiments}

In contrast to the wealth of theoretical studies on the adsorption behavior of bulk oxides, the progress on the experimental side is much smaller. This discrepancy is caused by the known conductivity problem connected with the investigation of oxide materials, but also reflects the 
difficulties to stabilize adsorbates on weakly-interacting supports and to circumvent their aggregation or desorption. Already the characterization of intrinsic oxide defects might be a challenge for many non-local experimental techniques. and exploring their role in adsorption events renders this task only more intricate. A distinction between regular and defect mediated adsorption schemes is generally deduced from $\operatorname{TDS}^{308,309}$ and micro-calorimetry measurements, ${ }^{310,332}$ exploiting the pronounced differences of the associated adsorption energies. The predominant interaction mechanism and the nature of the underlying defects remain, however, ambiguous in most of those studies. On the other hand, a local investigation of adsorption processes on bulk oxides is basically limited to $\mathrm{TiO}_{2}(110)$, as only this oxide provides sufficient electrical conductivity to perform STM experiments and can be prepared with high quality and reproducibility. ${ }^{40}$

Metal bonding to single-crystalline $\mathrm{TiO}_{2}$ surfaces was primarily investigated on the level of nano-particles. Hereby, mainly Au deposits have been in the focus of research, ${ }^{333}$ because of their unexpectedly-high catalytic activity for low-temperature CO oxidation. ${ }^{334}$ In experiments carried out at $300 \mathrm{~K}$, step edges were identified as the preferred nucleation sites for the metal atoms (Fig. 4.3A). ${ }^{335}$ The clusters initially grow two-dimensionally, but start to develop 3D shapes after reaching a critical size of approximately 40-50 atoms. Employing conductance spectroscopy with the STM, a non-metallic behavior was revealed for the 2D gold islands, characterized by a gap in their electronic states around the Fermi level of up to $1.5 \mathrm{eV}$ (Fig. 4.3B). ${ }^{333}$ This semiconductor-like electronic structure was suggested to be responsible for the good catalytic performance of the $\mathrm{Au} / \mathrm{TiO}_{2}$ system. ${ }^{333}$ Extensive studies on the particle morphology were later accomplished also for $\mathrm{Pd}$, Ag and bimetallic Ag-Au deposits on the $\mathrm{TiO}_{2}(110)$ surface. ${ }^{336,337,338}$

Reorganization and sintering of the ad-particles, following an Ostwald ripening mechanism, have been observed in STM experiments performed at elevated temperatures. ${ }^{339}$ The underlying material transport was found to be accelerated in the presence of molecular adsorbates, such as $\mathrm{CO}$ and $\mathrm{O}_{2}$, on the $\mathrm{TiO}_{2}$ surface. ${ }^{340}$ Annealing the particle/ $/ \mathrm{TiO}_{2}$ system above 900-1000 K results in encapsulation effects that means a thin oxide layer grows on top of the metal deposits. ${ }^{40,341,342}$ The process is driven by the drastic reduction of the surfacefree-energy upon covering the deposit by an oxide skin, and is commonly known as strongmetal-support interaction. For Pt and Pd particles, the encapsulation has been studied even at the atomic scale and a structure model for the oxide ad-layer could be derived from the STM data. $^{342}$ 

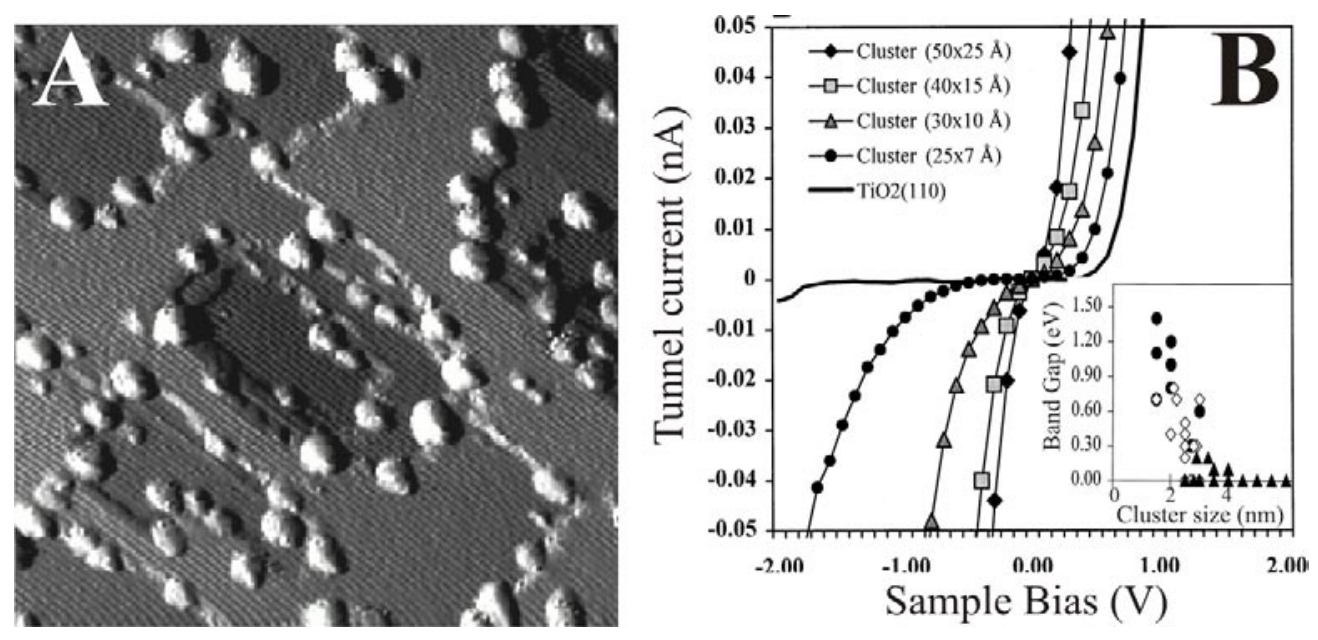

Fig. 4.3: (A) STM image of $0.25 \mathrm{ML}$ Au on $\mathrm{TiO}_{2}(110)\left(50 \times 50 \mathrm{~nm}^{2}\right)$. (B) Current-voltage spectra for Au clusters of different sizes. A region of zero-current becomes visible for the smaller aggregates, marking the gap in their electronic structure. The evolution of the gap value with cluster size is shown in the inset. ${ }^{333}$

For lower deposition temperatures, defects in the $\mathrm{TiO}_{2}$ surface become increasingly important for the nucleation of metal particles. The binding of single $\mathrm{Au}$ atoms and ultra-small aggregates on oxide terraces has been traced back to their interaction with oxygen vacancies in the bridging O-rows, being the dominant defect-type in the (110) surface (Fig. 4.4A). ${ }^{343}$ Every oxygen vacancy produces two under-coordinated $\mathrm{Ti}^{3+}$ ions, which are able to form relatively strong covalent bonds with an $\mathrm{Au}$ atom $\left(\mathrm{E}_{\mathrm{B}}=1.14 \mathrm{eV}\right)$. Correlating the number of oxygen vacancies covered by a single cluster to the cluster diameter suggests that each vacancy stabilizes only a limited number of Au atoms at the surface (roughly three atoms per vacancy). For larger Au-to-defect ratios, both the cluster and the anchoring vacancies start to diffuse on the surface at $\mathrm{T}>130 \mathrm{~K}$ until enough defects are collected to stabilize the cluster again. This surprising result was rationalized by the relatively small difference in the $\mathrm{Au}$ binding energy on a defect versus a regular surface site of only $0.45 \mathrm{eV}$ and by the high $\mathrm{Au}$ mobility on the oxide surface. ${ }^{343}$

More stable $\mathrm{Au}$ binding configurations were revealed on oxidized $\mathrm{TiO}_{2}(110)$ surfaces, being produced by $\mathrm{O}_{2}$ dissociation at $300 \mathrm{~K}$ (Fig. 4.4C). The oxygen treatment stimulates not only the healing of $\mathrm{O}$-vacancies, but leads to the formation of single $\mathrm{O}$ adatoms in addition. ${ }^{344}$ Oxide surfaces prepared in this way interact much stronger with $\mathrm{Au}$, as demonstrated by the high cluster density deduced from the STM measurements. According to associated DFT calculations, the $\mathrm{Au}$ attachment to oxidized $\mathrm{TiO}_{2}$ involves the formation of a highly polar $\mathrm{Au}-$ O bond accompanied by a charge transfer towards the oxygen. The resulting cationic gold is bound with $2.38 \mathrm{eV}$ to the surface, almost two times stronger than to the oxygen vacancy. A 
third route to modify the $\mathrm{Au}-\mathrm{TiO}_{2}$ interaction is hydroxylation of the surface via water exposure (Fig. 4.4B). ${ }^{344}$ A titania surface capped with OH-groups shows the smallest affinity to $\mathrm{Au}\left(\mathrm{E}_{\mathrm{B}}=0.54 \mathrm{eV}\right)$, and metal particles were found to nucleate exclusively along the oxide step edges.
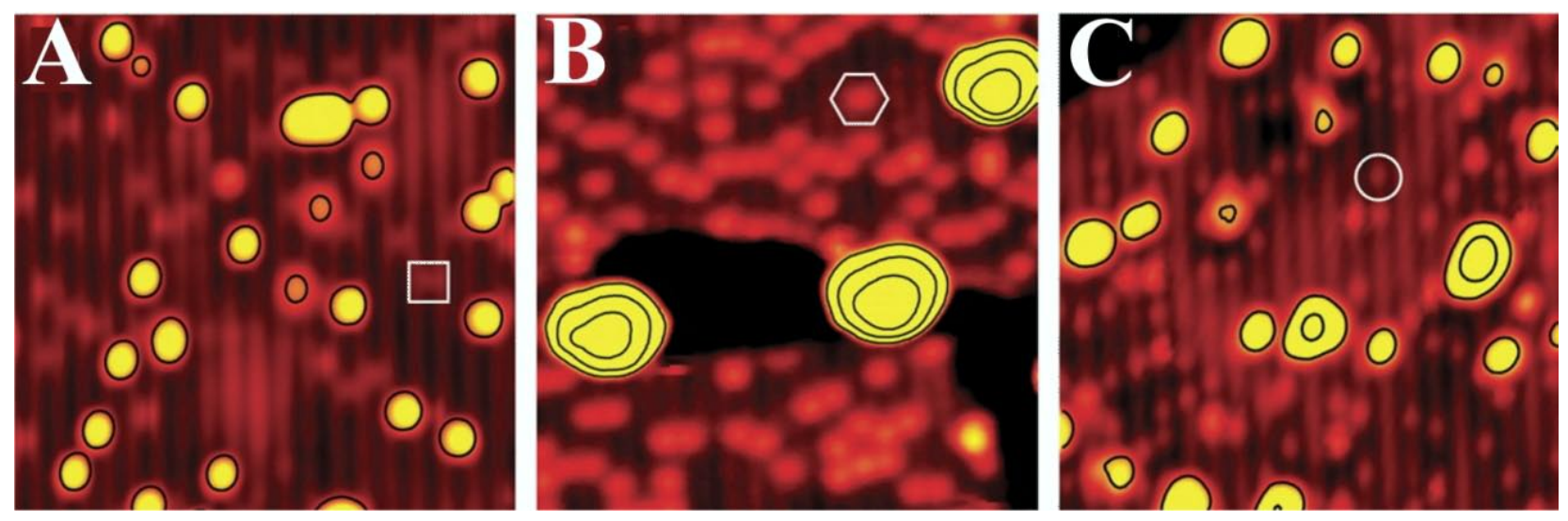

Fig. 4.4: STM images of (A) a reduced, $(\mathbf{B})$ a hydroxylated and $(\mathbf{C})$ an oxidized $\mathrm{TiO}_{2}(110)$ surface after deposition of 0.03ML Au at $300 \mathrm{~K}\left(13 \times 13 \mathrm{~nm}^{2}\right)$. The symbols indicate an $\mathrm{O}_{\text {bridge vacancy }}$ (square), an $\mathrm{H}$ atom on an $\mathrm{O}_{\text {bridge }}$ site (hexagon), and an $\mathrm{O}$ atom in the Ti troughs (circle). The smallest Au-titania interaction is observed for the hydroxylated surface in (B), leading to the largest Au particle sizes. $^{344}$

Another approach to fabricate well-defined Au aggregates on $\mathrm{TiO}_{2}(110)$ surfaces is the softlanding of mass-selected cationic clusters from the gas-phase. ${ }^{345}$ For kinetic energies below 3 $\mathrm{eV}$ per $\mathrm{Au}^{+}$ion, the oxide surface is not damaged and the adatoms are able to aggregate to metal particles in a similar way as observed for physical vapor deposition. At higher kinetic energy, the Au projectiles are pinned to the surface upon impingement and a high density of monatomic $\mathrm{Au}$ species is revealed in subsequent STM measurements. ${ }^{345}$ Comparable experiments demonstrated the versatility of this approach to produce larger aggregates with a distinct chemical identity and a defined number of atoms. Soft-landing of size-selected clusters therefore turned out to be a convenient way to fabricate $\mathrm{TiO}_{2}(110)$ surfaces with a high dispersion of metal deposits. ${ }^{346}$ Apart from the studies on $\mathrm{TiO}_{2}$, no STM experiments are reported in the literature that explicitly address the interaction of single adatoms with defects and regular sites in the surface of a bulk-oxide.

The experimental situation is similar what the adsorption of molecules on oxide surfaces concerns. Also here, comprehensive STM investigations have mainly been accomplished on the (110) and other $\mathrm{TiO}_{2}$ surfaces, reflecting the experimental advantages and the technological relevance of this particular system. The most extensive studies were performed 
on the adsorption and dissociation of water, being one of the key topics for hydrogentechnology and photo-catalysis using $\mathrm{TiO}_{2}$ as active material. ${ }^{5,347}$ The identification of water molecules and their dissociation products on the oxide surface has been a long standing puzzle, as intact molecules, surface hydroxyl groups and oxygen vacancies appear with similar contrast in the STM measurements (see also Fig. 3.15B). ${ }^{44,256,348}$ An unambiguous differentiation of the three species was possible only on modified surfaces, where either the concentration of defects was reduced by exposing the surface to oxygen or the hydrogen atoms were removed via electron-stimulated desorption during an STM high-voltage scan. ${ }^{257,349}$ The resulting assignment of the topographic features to oxygen vacancies, water molecules and OH-groups was later verified by DFT calculations that simulated their different appearance in the STM images. ${ }^{258}$
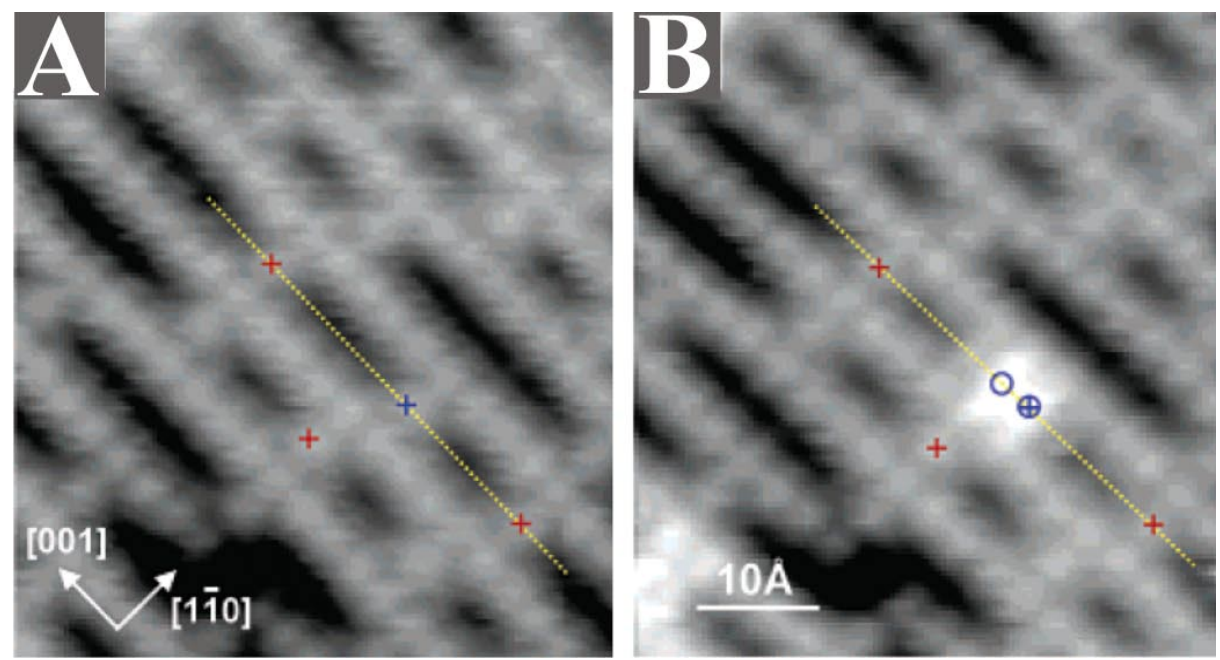

Fig. 4.5:

STM images obtained (A) before and (B) after adsorption of water on $\mathrm{TiO}_{2}(110)\left(+0.4 \mathrm{~V} ; 5 \times 5 \mathrm{~nm}^{2}\right)$. The + signs mark the positions of oxygen vacancies; the hatched and open circles depict the $\mathrm{OH}$ groups formed on the defect and on the adjacent oxygen site upon $\mathrm{H}_{2} \mathrm{O}$ dissociation.

On this basis, the dissociation of water molecules could be followed via in-situ STM measurements, which identified oxygen vacancies in the bridging O-rows as the active sites for water splitting. ${ }^{44,257,350}$ Subsequent studies even succeeded to visualize the different dissociation products of the heterolytic water splitting, comprising one OH-group that fills the initial vacancy and a second one forming on an adjacent bridging $\mathrm{O}$ atom (Fig. 4.5). ${ }^{351}$ From their diffusion behavior at room temperature, the inequivalence of the two surface $\mathrm{OH}$-groups was demonstrated. Whereas the hydroxyl group that includes an intrinsic lattice O-atom performs a diffusive motion with an activation energy of approximately $1.0 \mathrm{eV}$ (theoretical value is $1.5 \mathrm{eV}){ }^{350}$ the defect-bound hydroxyl group is essentially immobile at room 
temperature. The higher binding energy of the defective species is related to the residual charges localized at the two $\mathrm{Ti}$ atoms underneath, which are still in the $\mathrm{Ti}^{3+}$ oxidation state. The diffusion barrier for both kinds of $\mathrm{OH}$-species is substantially lowered when a water molecule approaches the hydroxyl group. The water-assisted motion in this case involves the temporal formation of a $\mathrm{H}_{3} \mathrm{O}^{+}$complex, which decays after finite time and leaves behind a surface OH-group in a new position. ${ }^{350,351}$

On reconstructed $\mathrm{TiO}_{2}(110)$, e.g. on the $(1 \times 2)$ added row reconstruction, the adsorption and dissociation of water is governed by different defect types than on the stoichiometric surface, e.g. by cross-links between two added titania rows. ${ }^{352}$

Several other molecules have been in the focus of STM adsorption experiments on $\mathrm{TiO}_{2}(110)$. Exposure of molecular oxygen was used to study the healing of vacancy sites in the oxide surface. ${ }^{257,353}$ The $\mathrm{O}_{2}$ molecule was hereby found to dissociate at temperatures as low as 120 $\mathrm{K}$, whereby one $\mathrm{O}$ atom filled the vacancy and the other one adsorbed on top of an exposed $\mathrm{Ti}$ row. The single $\mathrm{O}$ adatoms are strongly bound and unable to diffuse below room temperature. ${ }^{44}$ Sulfur adsorption was studied in a wide coverage and temperature range, disclosing a variety of adsorption geometries on the $\mathrm{TiO}_{2}$ surface. ${ }^{354}$ At low exposure and below $500 \mathrm{~K}$, the $\mathrm{S}$ atoms preferentially bind to the exposed $\mathrm{Ti}^{4+}$ rows in the (110) surface and arrange in a $(3 \times 1)$ superstructure. With increasing temperature, sulfur starts to replace the bridging oxygen atoms and later even the ones in the top-most bulk plane, whereby mainly [001]-oriented S-S pairs are incorporated into the surface. At $700 \mathrm{~K}$ adsorption temperature, almost $50 \%$ of the surface $\mathrm{O}$ atoms are substituted by sulfur in this way. The process of sulfidation is accompanied by the gradual reduction of the oxide surface and the appearance of a strong $\mathrm{Ti}^{3+}$ signal in XPS. ${ }^{354}$

In addition to inorganic species, ${ }^{355}$ several organic molecules have been investigated on $\mathrm{TiO}_{2}(110) .{ }^{356}$ Similar to water, methanol adsorbs dissociatively on oxygen vacancies. Upon dissociation, the defect is healed by the $\mathrm{O}$ atom abstracted from the methanol, leaving behind a methoxy group and a hydrogen atom adsorbed on an adjacent surface site. ${ }^{357}$ Also 2-butanol was found to dissociate on bridge-bonded oxygen vacancies by abstracting its OH-group. Only at high exposure and for a limited availability of defect sites, non-dissociated physisorbed species were detected, performing a rapid surface diffusion at room temperature. ${ }^{358}$ First STM experiments focusing on the photo-catalytic properties of $\mathrm{TiO}_{2}$ were performed with trimethyl acetic acid (TMAA). ${ }^{359}$ The molecule decomposes under UV- 
irradiation into trimethyl acetate and a surface hydroxyl group. The electrons released during the decomposition process become trapped at the $\mathrm{Ti}$ ion located below the $\mathrm{OH}$-groups and produce an increasing $\mathrm{Ti}^{3+}$ signal in electron-energy-loss spectra taken as a function of irradiation time. The change in the electronic structure of the molecule upon decomposition was not detected, although conductance spectroscopy with the STM would in principle be suited to monitor the molecular LDOS before and after reaction. This experimental goal has been achieved later for single Zinc-Etioporphyrin molecules adsorbed on the $\mathrm{TiO}_{2}$ surface, where changes in the electronic structure became evident in the $\mathrm{dI} / \mathrm{dV}$ spectra of a defectbound versus a regular ad-species. ${ }^{360}$
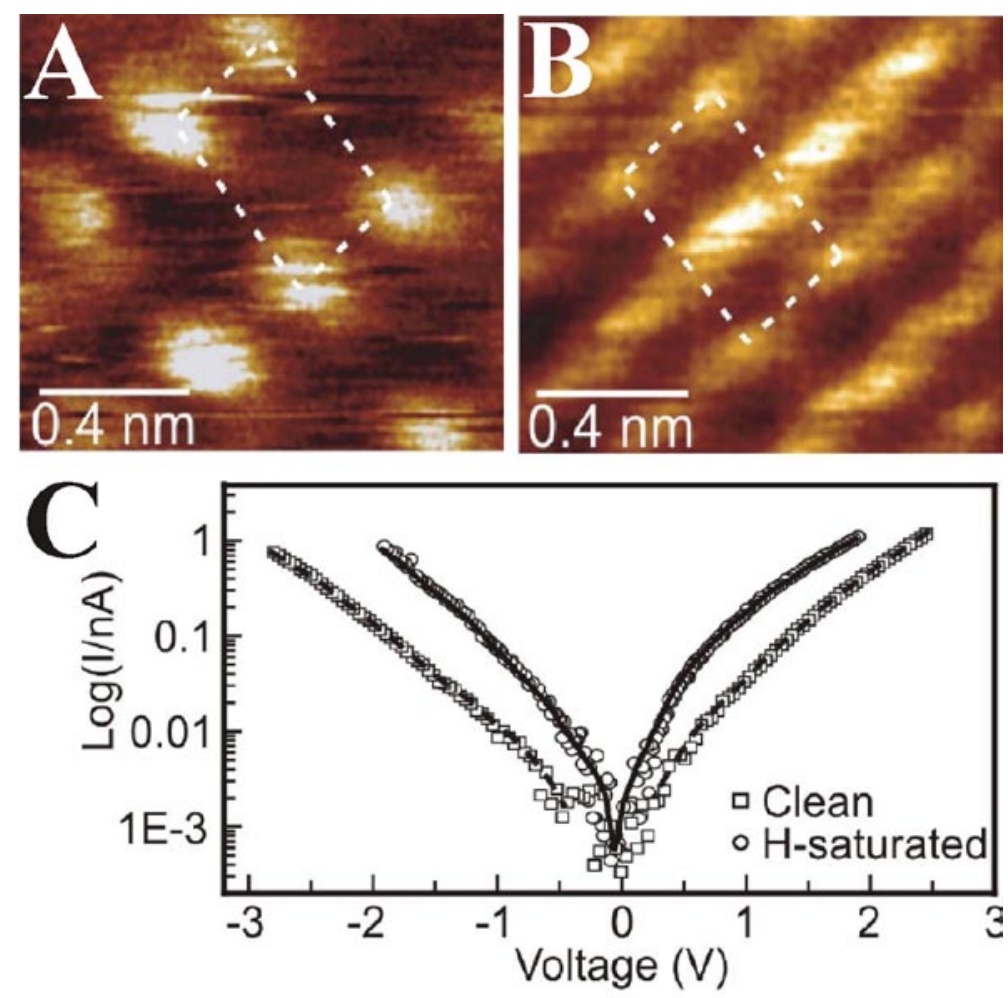

Fig. 4.6:

STM images of (A) a clean and (B) a hydrogen covered $\mathrm{ZnO}(10-10)$ surface. The dashed rectangle marks the $(1 \times 1)$ surface unit cell. Hadsorption at $300 \mathrm{~K}$ leads to a metallization of the $\mathrm{ZnO}(10-10)$ surface, as demonstrated by the vanishing zero-current region around $E_{F}$ in $I-V$ curves taken on both surfaces (C). ${ }^{35}$

Only a small number of other bulk oxides have been utilized besides titania in order to study adsorption processes with the STM. Detailed experiments were performed on the different surfaces of ZnO. Nucleation and growth of $\mathrm{Cu}$ has been explored on the polar $\mathrm{ZnO}(0001)$ surface, revealing the formation of 3D particles on the Zn-terminated, but 2D-wetting growth on the O-terminated surface due to the larger $\mathrm{Cu}-\mathrm{O}$ interaction in the latter case. ${ }^{361,362}$ On the non-polar $\mathrm{ZnO}(10-10)$, mainly the adsorption of hydrogen ${ }^{35,363}$ and water ${ }^{286,311,364}$ has been studied. Interestingly, binding of a single $\mathrm{H}$-atom per surface unit cell gives rise to a metallization of the initially semiconducting oxide, as demonstrated by STM conductance spectroscopy and HREELS (Fig. 4.6). The effect is explained by the transfer of the hydrogen electron into the $4 s$-band of zinc that becomes partly filled. The attachment of a second $\mathrm{H}$ - 
atom per unit cell, in contrast, gives rise to the complete filling of the surface $4 s$-state and the metallic character disappears again. ${ }^{35}$ Also the interaction of hydrogen with oxide defects has been addressed at the atomic level. ${ }^{363}$ Water exposure to the $\mathrm{ZnO}(10-10)$ leads to the formation of compact ad-islands with a $(2 \times 1)$ super-structure. ${ }^{311,364}$ Based on atomically resolved STM images and DFT calculations, the ad-islands were found to consist of 50\% associatively-bound $\mathrm{H}_{2} \mathrm{O}$ molecules and $50 \%$ dissociated species, forming two $\mathrm{OH}$ groups with an $\mathrm{O}$ and a $\mathrm{Zn}$ surface atom, respectively. The fact that water partly dissociates on the $\mathrm{ZnO}(10-10)$ surface is compatible with a binding energy of more than $1.1 \mathrm{eV}$ for the isolated molecule, which is an exceptionally large value for oxide surfaces. ${ }^{311}$ The strength of the $\mathrm{H}_{2} \mathrm{O}-\mathrm{ZnO}$ bond in this case results from the large $\mathrm{O}_{\mathrm{H} 2 \mathrm{O}}$ interaction with an unsaturated surface $\mathrm{Zn}$ atom and the formation of two $\mathrm{H}$-bonds with a substrate $\mathrm{O}$ atom and a neighboring water molecule. $^{311}$

Other adsorption experiments with the STM have been carried out on $\mathrm{Fe}_{3} \mathrm{O}_{4}(111)^{365}$ and anatase $(001)^{366}$ bulk samples, investigating the interaction of water and carboxylic acid with the oxide surface, respectively. Although in some of these studies, different adsorption pathways and the role of defects could be analyzed, the quality and quantity of the experiments did not lead to the same level of understanding as for the $\mathrm{TiO}_{2}$ and $\mathrm{ZnO}$ surfaces. Finally, STM-conductance spectroscopy is hardly applied to explore adsorption processes on bulk oxides, despite of the unique capabilities of the technique to probe the electronic structure of single oxide-adsorbate complexes.

In contrast to bulk oxides, a wealth of experimental results is available for thin oxide films grown on metal supports. The adsorption behavior of such systems will be discussed in the following chapters.

\subsection{Adsorption on thin oxide films - Theory}

The adsorption behavior of oxide thin films differs from the one of bulk materials when the film thickness reaches the length scale of typical oxide-adsorbate interactions and the metal support starts to influence the binding potential of the oxide surface. As different adsorption schemes are characterized by different interaction lengths, the suitability of an oxide film to serve as model system for the respective bulk material depends on the nature of the binding mechanisms. Chemisorption of adatoms and molecules is usually associated with the formation of a true chemical bond and consequently restricted to a few neighboring atoms 
around the adsorption site. For strongly bound ad-species or spatially confined interactions with a lattice defect, oxide films of 5-10 ML thickness might thus be sufficient to reproduce the binding characteristic of the bulk system. On the other hand, coupling mechanisms mediated by polarization and Coulomb forces are often long-ranged and decay on a length scale of a few nanometers. In this case, the thin-film adsorption converges towards the bulk behavior only for a layer thickness of more than $10 \mathrm{ML}$, depending on the screening ability of the oxide material. ${ }^{36}$ For oxide films below this critical thickness, the influence of the metal support has to be taken into consideration to accurately describe the attachment of atomic and molecular species. ${ }^{186}$

The presence of the metal support affects the adsorption properties of an oxide film in various aspects, most of them resulting in an enhanced binding strength with respect to the bulk material (Fig. 4.7). Metals have in general a much higher static and dynamic polarizability than oxides, reflecting their free-electron character and strong dielectric response. Consequently, adsorbates on thin oxide films experience larger dispersive or van-der-Waalslike forces than on bulk oxides, as those binding contributions are enhanced by the metal underneath. ${ }^{165}$ A special case is the coupling between adsorbates that carry a net-charge and their image-charge induced in the metal support. Such electrostatic interactions decay only with the inverse film thickness $\left(d^{-1}\right)$, compared to the $d^{-6}$ dependence revealed for the dispersive interactions between neutral species They are hence extremely efficient to stabilize charged adsorbates even on thicker oxide layers. The increased polarizability of the system, as introduced by the metal support, affects in particular the binding of those adsorbates that are not able to form a true chemical bond with the oxide film, e.g. closed-shell molecules and noble and near-noble metal atoms. ${ }^{367}$

Closely related to polarization interactions is the possibility to exchange charges through the oxide film. ${ }^{165,186,368,369}$ The supporting metal represents an infinitely large electron reservoir and is therefore able to release electrons into electronegative or accept electrons from electropositive adsorbates (Fig. 4.7). The charged ad-species that is generated upon electron transfer features several new coupling mechanisms to the oxide surface, leading to a substantially increase of its adsorption energy. In the case of ionic oxide materials, the adsorbate is subject to a Coulomb interaction with the oxide Madelung potential and binds either to surface anions or cations, depending on its charge state. The preferred binding site on a thin film might therefore differ from the one on the bulk crystal where the adsorbate remains neutral. Furthermore, a charged ad-species is able to initiate a polaronic distortion in the 
underlying lattice, which means that the oxide ions next to the adsorption site are displaced in order to accommodate the extra charge. ${ }^{370}$ A polaronic distortion is the typically response of an ionic system to a charge-perturbation and plays not only a role for adsorption processes, ${ }^{88,371}$ but also for the stabilization of excitons in oxide materials. ${ }^{130}$ Finally, an image charge is induced in the metal support below, which further increases the interaction strength with respect to the neutral species, as discussed before. To give an estimate for the efficiency of charge-induced interaction schemes, the Au-MgO binding energy increases from $0.9 \mathrm{eV}$ for the neutral adatom on bulk $\mathrm{MgO}$ to $2.3 \mathrm{eV}$ for an $\mathrm{Au}^{-}$species on a $3 \mathrm{ML}$ thick $\mathrm{MgO} / \mathrm{Mo}(001)$ film. ${ }^{186}$

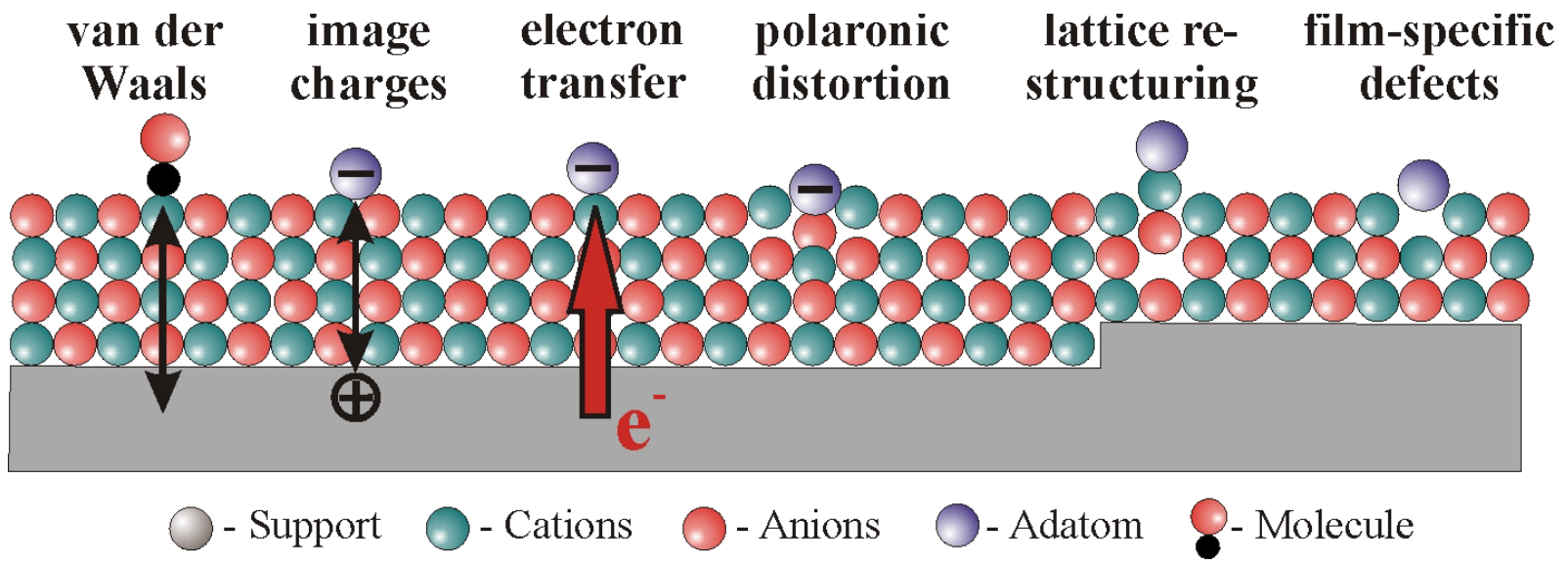

Fig. 4.7: Examples for binding mechanisms which are specific to thin-film systems.

Several preconditions need to be fulfilled before electrons can be exchanged between an adsorbate and the metal substrate below the oxide film. ${ }^{165,186,370}$ For negative charging, the adsorbate has to possess a low-lying affinity level that is able to shift below the Fermi energy of the metal-oxide system upon adsorption (Fig. 4.8). Such down-shift of the adsorbate electronic states might be induced by a work-function decrease of the support, which often accompanies the growth of oxide films on metal supports (see Chapter $\underline{3.2}$ ) ${ }^{187}$ The reduced work function lowers the vacuum energy at the oxide surface and pushes unfilled adsorbate states towards $E_{F}$. If they cross the Fermi energy during this process, the levels become filled due to an electron transfer from the metal substrate, provided that charge transport through the oxide film is enabled e.g. via tunneling. The reverse process, resulting in the formation of cationic surface species, requires the presence of filled adsorbate levels in vicinity to $E_{F}$ and an increase of the metal-oxide work function. ${ }^{372}$ In several aspects, the electron exchange with adsorbates on the oxide surface is comparable with the surface oxidation of metals, as proposed by Cabrera and Mott. ${ }^{373}$ Also the oxidation process requires a transfer of electrons 
from the metal bulk to the oxide surface and only proceeds as long as the charge transport through the growing oxide film is maintained.

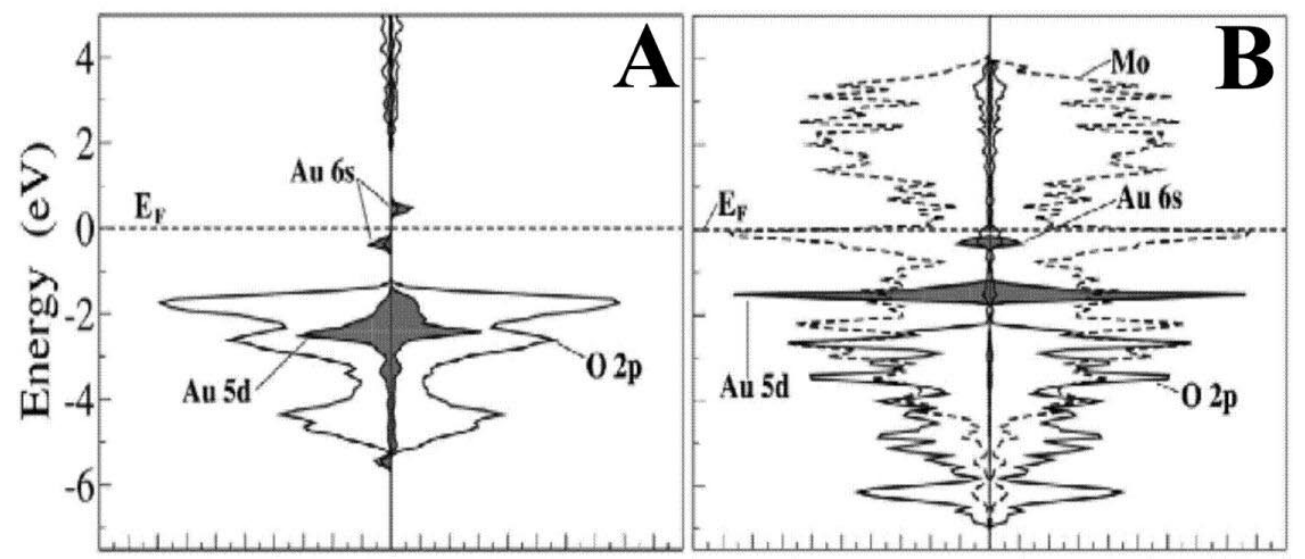

Fig. 4.8:

Calculated LDOS of an Au atom on (A) bulk MgO and (B) a 3ML thick MgO/Mo(001) film. While the $A u$ ss state remains half-filled on the bulk oxide, it shifts below the Fermi level on the thin-film system, indicating a charge transfer from the metal support. ${ }^{186}$

The importance of charge transfer processes for the adsorption characteristic of thin oxide films was first realized for Au interacting with a 3ML thick MgO film on $\mathrm{Ag}(001) .{ }^{186,374}$ The charge transfer into the Au adatom occurs, because the $A u$ 6s orbital that is half-filled in the gas-phase species is pushed below the Fermi level upon adsorption and fills up with an extra electron from the Ag-MgO interface (Fig. 4.8). The electron flow into the Au is facilitated by a work function decrease of more than $1.0 \mathrm{eV}$, being induced by the $\mathrm{MgO}$ growth on the Ag(001) surface. ${ }^{187}$ The $\mathrm{Au}^{-}$species binds two times stronger to the oxide film than its neutral counterpart and interchanges its preferred $\mathrm{O}^{2-}$ binding sites on bulk $\mathrm{MgO}$ to an $\mathrm{Mg}^{2+}$ site on the thin film. In contrast, charge-induced binding schemes are not active for Pd atoms on the same system, because Pd does not have a suitable affinity level to be pushed below $\mathrm{E}_{\mathrm{F}}$ upon adsorption. ${ }^{186}$ The importance of charge-transfer processes to stabilize adsorbates on oxide thin films was later realized for other systems, e.g. for $\mathrm{Au}$ atoms on alumina/ $\mathrm{NiAl}(110)^{375}$ and $\mathrm{FeO} / \mathrm{Pt}(111)^{372}$ as well as for ultra-small Au clusters on thin MgO films. ${ }^{376,377}$ In principle, the charge state of an adsorbates might even be adjusted in a desired way by controlling the work function of a metal-oxide system and therewith the direction of the charge-flow. Theoretical simulations have demonstrated that work function tuning of thin oxide films is feasible by inserting a sufficient number of donors or acceptors. ${ }^{371,378}$ For instance, the embedding of $\mathrm{K}$ and $\mathrm{Li}$ atoms into a silica thin film produces positive charge centers that sufficiently reduce the oxide work function to stimulate a charge transfer into adsorbed Au species. ${ }^{379}$ 
Apart from the possibility to exchange electrons, thin oxide films have a higher flexibility to form true chemical bonds with an adsorbate by rearranging their internal atom configuration and bond structure (Fig. 4.7). While bulk oxides usually exhibit an extremely high energybarrier for breaking and reorganizing surface bonds, such restructuring is less activated in thin films due to the balancing influence of the metal support. A bond rearrangement might now be able to free new valences for stabilizing adatoms and molecules on the originally inert oxide surface, as first demonstrated for the Au interaction with alumina/ $\mathrm{NiAl}(110)$ thin films. ${ }^{375}$ In this example, attachment of a single Au atom induces the cleavage of an Al-O bond in the film, which in turn enables the formation of a new bond between the hence unsaturated $\mathrm{Al}$ ion and the adatom (see also Fig. 4.11). The presence of an under-coordinated $\mathrm{O}$ ion is prevented in this case, as the oxygen species is re-bound to an $\mathrm{Al}$ atom in the metal surface below the oxide film. Thanks to this bond reorganization, the Au-alumina adsorption energy increases to $2.1 \mathrm{eV}$, which compares to $0.8 \mathrm{eV}$ for bulk $\mathrm{Al}_{2} \mathrm{O}_{3}(0001) \cdot{ }^{380} \mathrm{~A}$ similar mechanism has been predicted for the Au adsorption on Ti-doped silica films. Also here, a Ti$\mathrm{O}$ bond is broken in order to form a new Ti-Au bond. ${ }^{381}$ The loss of coordination for the oxygen ion is counterbalanced by the high Au adsorption energy and the strengthening of the $\mathrm{O}$ bond to the $\mathrm{Mo}(112)$ support below the oxide film. Also the $\mathrm{Au}$ interaction with FeO films grown on $\mathrm{Pt}(111)$ is expected to involve the rupture of an internal bond. ${ }^{372}$ In the energetically most favorable binding geometry, a Fe-Pt bond at the interface is broken and the Fe atom moves to the oxide surface, where it binds the Au adatom much stronger than the originally oxygen-terminated FeO surface (Fig. 4.9). However, the proposed binding configuration could not be verified experimentally, most likely because of the large kinetic barrier to activate the Fe-Pt bond cleavage. ${ }^{372}$

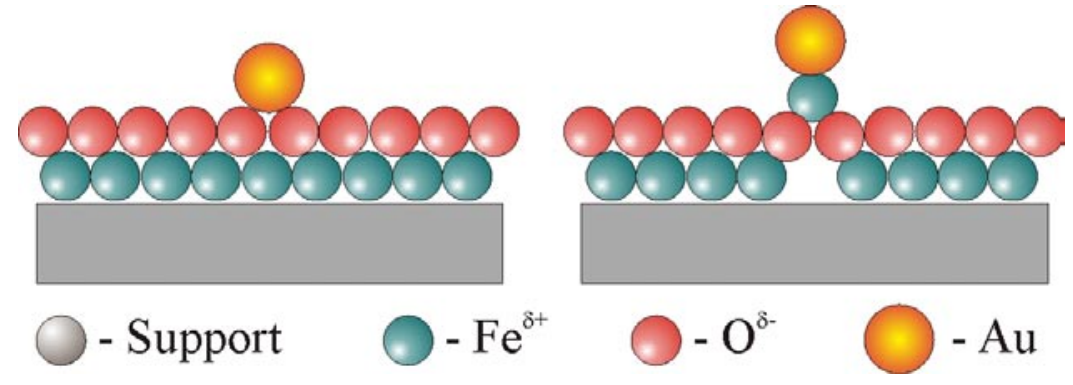

Fig. 4.9:

Experimentally observed (left) and calculated binding configuration (right) for an Au atom on the $\mathrm{FeO} / \mathrm{Pt}(111)$ film. In the energetically favorable scenario, one Fe atom flips above the oxide plane in order to increase the interaction with the $\mathrm{Au}{ }^{372}$

Similar to the situation encountered for bulk oxides, surface defects play an important role for anchoring adsorbates on oxide thin films. However, some important deviations have to be 
taken into account when discussing defects in thin-film systems. First, the difference in the adsorption energy between a regular and a defect site is usually smaller than on bulk oxide surfaces, reflecting the binding contribution of the metal substrate and its screening response to a defect-induced electronic perturbation. Second, many defects in thin films are directly related to its growth on a substrate with deviating lattice parameter, and have consequently no counterpart in the respective bulk material. ${ }^{51,210,212}$ A number of examples has already been given in Chapter $\underline{3.5}$ and only misfit-induced dislocation lines should be mentioned again. And third, even when identical defects occur in bulk and thin film oxides, their binding potential might be different because of a deviating electronic structure. For example, while color centers in bulk materials are usually filled with one or two electrons, ${ }^{267}$ they might be empty in thin films as a result of an electron transfer into the metal underneath. ${ }^{195}$

The binding potential of defects in oxide thin films has been illuminated only in a few cases from the theoretical point of view, for instance for oxygen vacancies in $\mathrm{SiO}_{2} / \mathrm{Mo}(112)$ or $\mathrm{MgO} / \mathrm{Mo}(001) .{ }^{195,328,368}$ In analogy to bulk defects, the binding energy is higher than on regular lattice sites, reflecting the chemically unsaturated nature of the defect. Vacancies in a vanadyl-terminated $\mathrm{V}_{2} \mathrm{O}_{3}$ film seem to be an exception from this general rule. ${ }^{262}$ Here, the binding of $\mathrm{Au}$ to a $\mathrm{V}=\mathrm{O}$ vacancy site was found to be weaker than to a regular surface site. Only upon dissociation of a $\mathrm{V}=\mathrm{O}$ group, a chemically unsaturated and hence reactive $\mathrm{V}$ atom is produced in the surface that strongly interacts with the Au. ${ }^{262}$ For more detailed information, the literature on respective bulk oxides needs to be consulted, ${ }^{18,19}$ keeping in mind that the role of defects in thin films might differ from the one in bulk materials in quantitative as well as qualitative manner.

In summary, oxide films of a few nanometer thickness are well suited to mimic the adsorption behavior of the respective bulk oxides. With decreasing layer thickness, the influence of the metal support increases, leading to an overall enhancement of the oxide-adsorbate coupling. In the limit of ultra-thin films, new adsorption mechanisms emerge due to the possibility to exchange electrons through the oxide layer. As an interesting side-effect, the charge state of adsorbates becomes controllable in such systems, e.g. by modifying the oxide thickness and support material or by embedding suitable dopants into the film. These possibilities open new routes to design the adsorption behavior and closely related the catalytic performance of thinfilm systems, which are not available in bulk oxides. In the following chapter, state-of-the-art STM experiments are reviewed that deal with the adsorption properties of thin oxide films on metal supports. 


\subsection{Adsorption on thin oxide films - Experiments}

Adsorption experiments with the STM have been performed with metal adatoms as well as molecules on various thin oxide films. The following discussion does not describe the different adsorption systems one by one, but organizes them in accordance to the dominant interaction mechanism between adsorbate and thin-film support. The focus of the next chapter lies on adsorption phenomena that are accompanied by an electron transfer through the oxide film and the formation of charged ad-species. The underlying theoretical concept has already been introduced in Chapter $\underline{4.3}$.

\subsubsection{Charge-mediated adsorption schemes}

\section{Single adatoms}

The very first experimental indication for the presence of charged adsorbates on oxide thin films came from low-temperature (5 K) STM studies on $3 \mathrm{ML} \mathrm{MgO/Ag(001)} \mathrm{exposed} \mathrm{to}$ small amounts of $\mathrm{Au} .{ }^{374}$ In contrast to the strong tendency of gold to aggregate on bulk oxides, single atoms were identified as the dominant ad-species on the film surface even for relatively high $\mathrm{Au}$ exposures. The adatoms self-assembled into a hexagonal pattern, whose lattice parameter was exclusively determined by atom density on the surface and not by the MgO crystallographic structure (Fig. 4.10). The required atom mobility for the ordering process was traced back to the transient thermal energy of the incoming adsorbates. The distinct spatial distribution of the Au atoms on the $\mathrm{MgO}$ thin film can be taken as an evidence for their charged nature. The equally-charged ad-species experience a repulsive Coulomb interaction, which is reduced by maximizing the inter-atomic distance between the atoms and results in their self-organization into a hexagonal lattice. A similar ordering effect was earlier observed for alkali atoms on metal and semiconductor surfaces and attributed to the positive charging upon adsorption. ${ }^{382,383}$ The Au atoms, on the other hand, charge up negatively, as their $A u$ 6s orbital fills up with an extra electron from the $\operatorname{Ag}(001)$ below the oxide film (see discussion in Chapter $\underline{4.3}$ ). The concomitant activation of charge-dependent binding schemes leads to a substantial increase of the Au-MgO interaction and is the driving force for the charge exchange. ${ }^{186}$ The direction of the charge flow is given by the electronegative character of gold in conjunction with the small Ag-MgO work function, ${ }^{187}$ both supporting an electron 
transfer into the $\mathrm{Au}$. The experimentally deduced charging mechanism is in perfect agreement with DFT calculations that revealed a Bader charge of approximately -1|e| for the Au atoms. ${ }^{186}$ Further evidence for the charged nature of $\mathrm{Au}$ on $3 \mathrm{ML} \mathrm{MgO/Ag(001)} \mathrm{comes} \mathrm{from} \mathrm{the} \mathrm{distinct}$ sombrero shape of individual adatoms, whereby a dark ring surrounds the actual Au-induced protrusion in low-bias STM images (Fig. 4.10A, inset). Sombrero-like features are considered as a fingerprint for charge-impurities located on insulating and semi-conducting surfaces and have been observed for charged atoms on $\mathrm{NaCl}$ thin films and $\mathrm{Si}(111),{ }^{88,384}$ as well as for impurity species in $\mathrm{TiO}_{2}(110)$ bulk samples. ${ }^{259}$ The particular shape of the adsorbate in the STM is hereby caused by a local bending of the host bands in vicinity to the point charge. On thin insulating films, this effect interferes with the scattering pattern of the conduction electrons in the metal support below the charge-impurity.
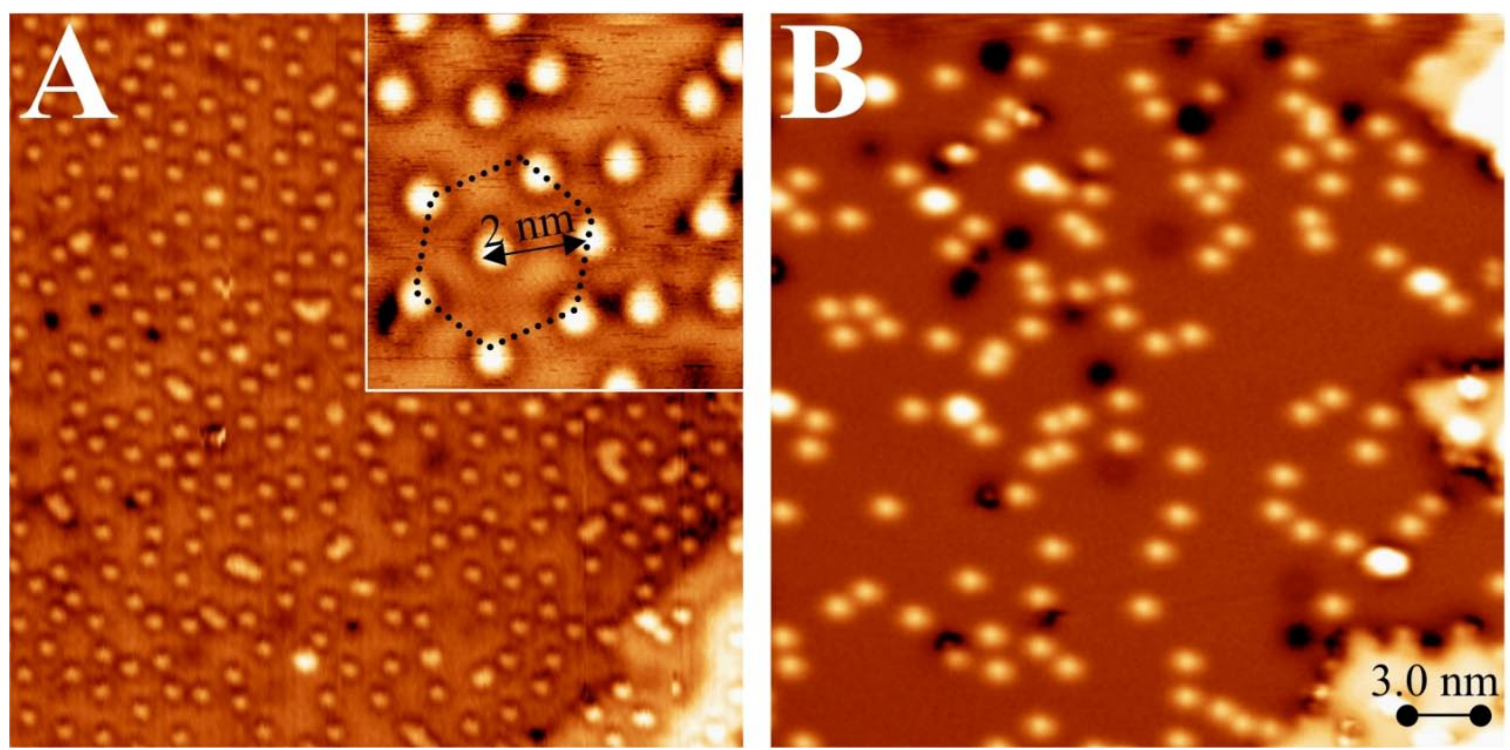

Fig. 4.10: Low-temperature STM image of (A) single Au and (B) single Pd adatoms on a 3 ML thick MgO film grown on $\mathrm{Ag}(001)\left(30 \times 30 \mathrm{~nm}^{2}\right)$. While the negatively charged Au atoms self-assemble into a hexagonal pattern (see inset), the neutral Pd atoms are randomly distributed on the oxide surface and show a large tendency to aggregate into clusters. A negative charge on the Au species is also compatible with the distinct sombrero shape observed in low-bias STM images. Black dots in both images are attributed to defects in the oxide film. ${ }^{374}$

The ability to exchange electrons with the support upon adsorption depends on the electronic structure and chemical identity of the adatom. In contrast to Au, Pd atoms do not possess a low-lying affinity level. The Pd $5 s$ state, as the lowest unoccupied atomic orbital in the gasphase atom, remains well above the Fermi level also after Pd deposition onto the $\mathrm{MgO} / \mathrm{Ag}(001)$ film. Consequently no electron transfer takes place and the adatom keeps its neutral charge state on the oxide surface. ${ }^{186}$ The absence of charging is clearly revealed from the spatial arrangement of the $\mathrm{Pd}$ on the MgO film (Fig. 4.10B). ${ }^{374,385}$ The adatoms are 
randomly distributed on the surface and exhibit a high tendency to aggregate even at low atom densities. Both observations suggest the absence of repulsive inter-atomic forces that would keep the atoms separated. Furthermore, Pd atoms are imaged as simple Gaussian maxima at all sample voltages, and no sombrero shape as a fingerprint of a potentially charged species is resolved.

A charge-mediated adsorption behavior was not only observed for $\mathrm{Au}$ atoms on $\mathrm{MgO}$ thin films, but also on other thin-film systems. ${ }^{372,375}$ Particularly interesting in this context is the combined STM-DFT study on the Au interaction with alumina/NiAl(110) films, because the direction of the charge flow could directly be deduced in this case from the experimental data. ${ }^{386}$ The structural properties of the four-layer alumina film have been discussed in detail in Chapter 3.2 (see Fig. 3.5); ${ }^{31}$ and only the film thickness of $5 \AA$ should be mentioned again as it is comparable with the value of 2-3 ML MgO. Although the charge transfer between the $\mathrm{Au}$ atoms and the $\mathrm{NiAl}$ prevails as the dominant binding mechanism, details of the interaction considerably deviate from the $\mathrm{MgO} / \mathrm{Ag}(001)$ case and include a bond reorganization in the oxide film upon $\mathrm{Au}$ attachment. ${ }^{375}$ The $\mathrm{Au}$ atoms exclusively bind to the $\mathrm{Al}_{\mathrm{s}}^{3+}$ cations in the surface, while interaction with the $\mathrm{O}_{\mathrm{s}}^{2-}$ sites does not lead to stable binding configurations (Fig. 4.11, inset). Upon bond formation, the $\mathrm{Al}$ ion is lifted above the surface plane, increasing the bond length to the $\mathrm{O}_{\mathrm{i}}$ ion underneath from 1.8 to $4.4 \AA$. As a result, this particular $\mathrm{Al}_{\mathrm{s}}-\mathrm{O}_{\mathrm{i}}$ bond in the film breaks and the $\mathrm{Al}$ transfers one of its three valence electrons to the $\mathrm{Au}$ and not the $\mathrm{O}_{\mathrm{i}}$ atom as before. Particularly stable binding configurations are now revealed, when the under-coordinated $\mathrm{O}_{\mathrm{i}}$ ion below the Au adsorption site forms a new bond to an $\mathrm{Al}$ atom in the $\mathrm{NiAl}(110)$ and is able to replenish its charge state via an electron transfer from the support. In this case, the Au-alumina binding energy increases to $2.1 \mathrm{eV}$, compared to values below $1.5 \mathrm{eV}$ without participation of the NiAl substrate. Due to the incommensurate relationship between alumina and $\mathrm{NiAl}(110),{ }^{177}$ only a limited number of $\mathrm{Al}_{\mathrm{s}}$ surface ions are directly located on-top of an $\mathrm{Al}$ atom in the metal and thus able to promote this special binding mechanism. However, the large energetic preference for the NiAl-assisted adsorption mechanism renders those binding sites crucial for the nucleation and growth behavior of $\mathrm{Au}$ on the alumina film. It should be added that the Au-induced bond cleavage in the alumina film is only an extreme form of a polaronic lattice distortion and occurs to a smaller extent also in the $\mathrm{MgO}$ thin film upon $\mathrm{Au}^{-}$attachment. 

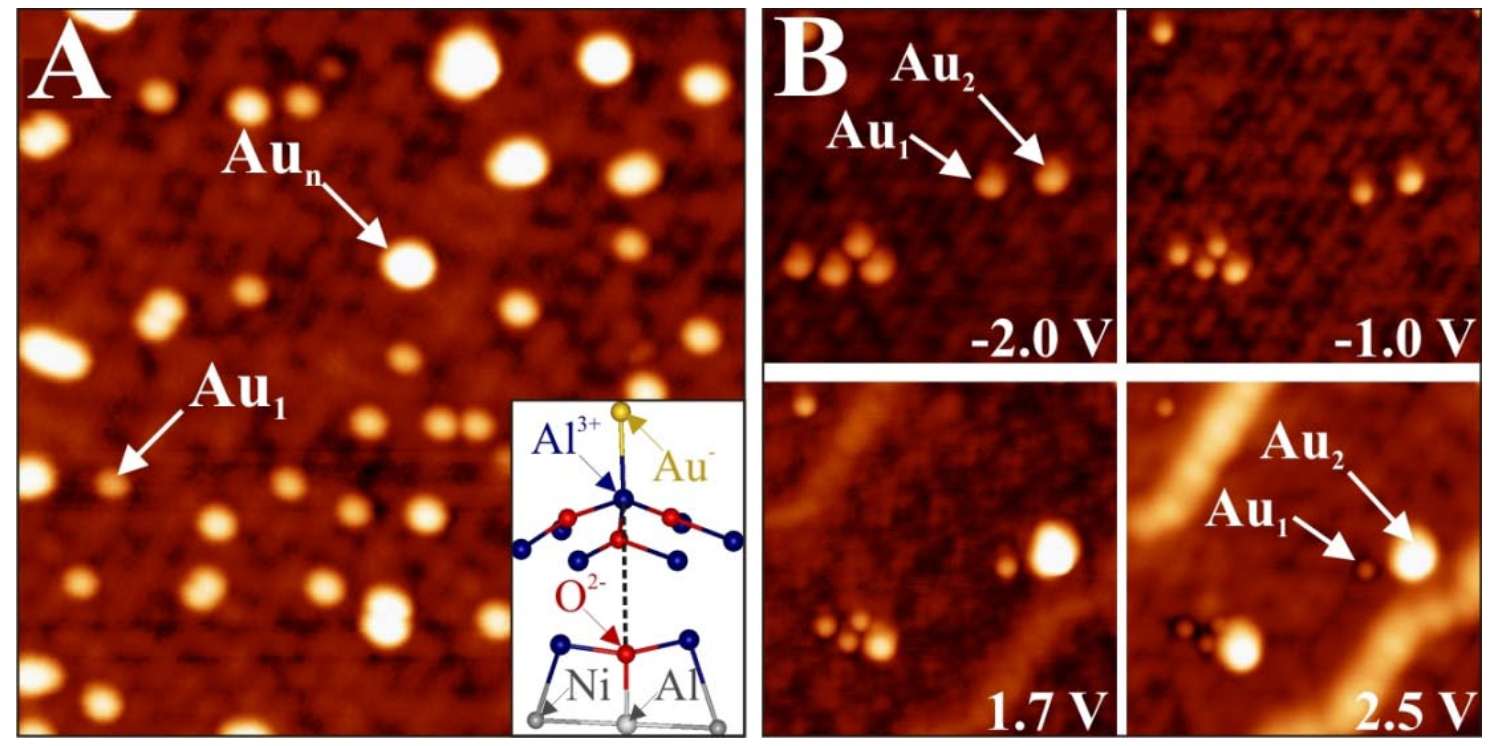

Fig. 4.11:

(A) STM image of Au monomers and small aggregates on alumina/NiAl(110) $\left(-1.5 \mathrm{~V}, 18 \times 18 \mathrm{~nm}^{2}\right)$. The inset shows a ball-stick model of a monomer bound to an Al surface ion. (B) Image series of a small alumina region at different bias voltages $\left(15 \times 15 \mathrm{~nm}^{2}\right)$. The various Au species undergo large contrast changes as a function of the imaging conditions. ${ }^{386}$

The $\mathrm{Au}$ adsorption on special $\mathrm{Al}_{\mathrm{s}}^{3+}$ binding sites and the resulting negative charging is compatible with low-temperature STM measurements on the alumina/NiAl(110) system (Fig. 4.11A). Single Au atoms appear as circular protrusions of $6 \AA$ diameter located on-top of the $\mathrm{Al}_{\mathrm{s}}$ sub-lattice, as deduced from atomically resolved STM images. ${ }^{387}$ The adatoms are randomly distributed on the alumina surface, in contrast to the hexagonal arrangement observed on $\mathrm{MgO} / \mathrm{Ag}(001) .{ }^{374}$ The absence of long-range order is caused in this case by pronounced spatial modulations in the adsorption energy landscape, reflecting the different local contributions of the $\mathrm{NiAl}$ support to the Au-alumina interaction. Nonetheless, the charged nature of the $\mathrm{Au}$ is clearly derived from the pronounced contrast reversal of the adspecies as a function of imaging bias (Fig. 4.11B). At negative bias (probing the occupied sample states) an Au monomer is imaged as a 1 Å high protrusion. This height value gradually decreases with increasing positive bias and even turns negative above $+3.0 \mathrm{~V}$, when the $\mathrm{Au}$ atom appears as a shallow depression in the alumina surface (Fig. 4.12A). At intermediate bias voltages (between +2.0 to $+3.0 \mathrm{~V}$ ), the $\mathrm{Au}$ atoms are imaged with the characteristic sombrero shape of a charged ad-species (Fig. 4.11).

According to the Tersoff-Hamann theory, a negative apparent height manifests a lower state density in the adatom than in the surrounding oxide, which forces the tip to approach the surface in order to maintain the preset tunnel current. ${ }^{68}$ This implies that the Au atom has no electronic states by its own to promote electron transport at elevated positive bias and its 
lowest-unoccupied orbitals (the LUMO) is not yet reached. However, the Au even seems to remove state density from the oxide patch underneath, because tunneling through the adatom is less efficient than through the bare oxide film. This behavior can be rationalized by a local bending of the alumina bands in response to the Au ion that acts as a charge-impurity on the oxide surface. ${ }^{384}$ At the bias value of the contrast reversal, additional states become available in the unperturbed alumina due to the onset of the oxide conduction band. ${ }^{193}$ Around the adatom, however, this band onset is shifted to higher energies and the gap situation with its negligible state density is maintained, giving rise to the negative contrast. Such upward bending is only compatible with a negatively charged species that exerts a repulsive interaction on the alumina electronic states. ${ }^{94}$ Positive surface charges, on the other hand, would induce a downward bending in contrast to the observations. Assuming a screened Coulomb interaction of: $V=-\frac{q}{4 \pi \varepsilon_{0} \varepsilon_{r} r}$, with an alumina dielectric constant of $\varepsilon_{r}=10$ and an Au excess charge of $q=-1|\mathrm{e}|$, the upward bending calculates to $+1.8 \mathrm{eV}$ in $r=1 \AA$ radius around the anion. ${ }^{94}$ This value agrees well with the bias window of 3.0 to $4.5 \mathrm{~V}$, in which the $\mathrm{Au}$ atom appears with negative contrast in the STM topographic images. The contrast reversal observed for $\mathrm{Au}$ atoms on alumina thin films thus provides independent evidence for the charge-transfer mechanism that accompanies the interaction of gold with many oxide films. ${ }^{186}$ For Au dimers, the contrast reversal is not observed, because an intrinsic electronic state of the $\mathrm{Au}_{2}$ dominates the $\mathrm{dI} / \mathrm{dV}$ signal in the decisive bias range around the conduction band onset. As a result, the dimer appears bright in STM images taken above $2.5 \mathrm{~V}$, as demonstrated in Fig. 4.11 and 4.12.
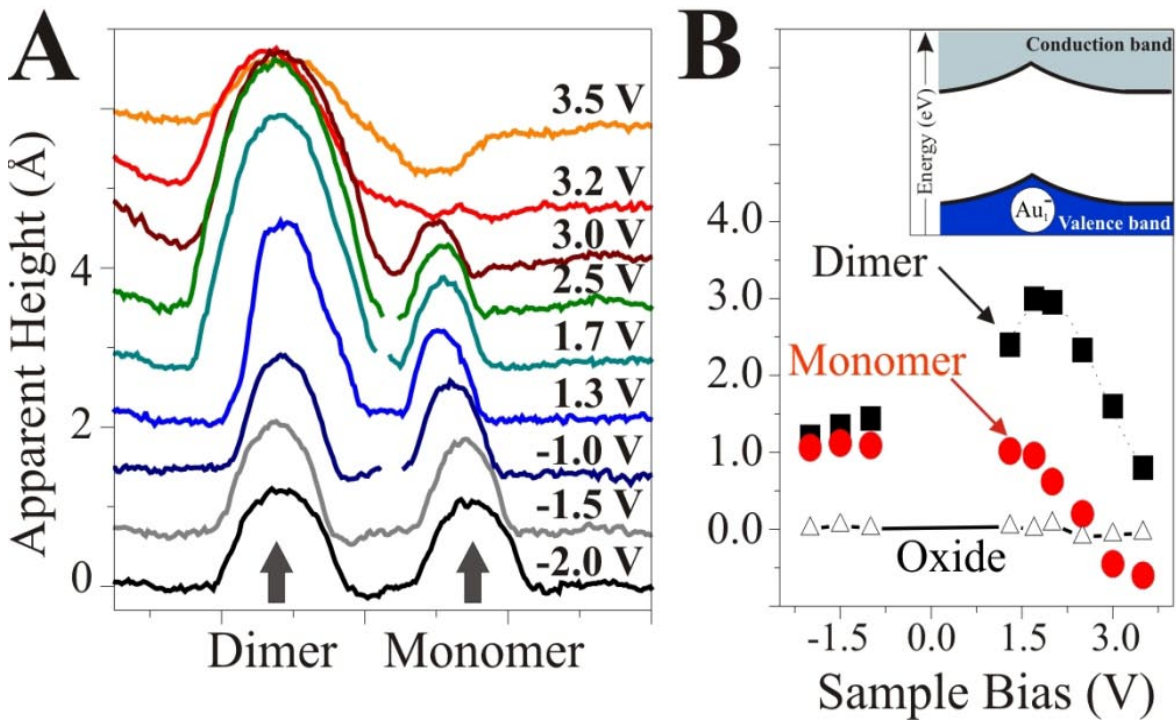

Fig. 4.12:

(A) Line profiles and (B) apparent heights of an Au monomer and a dimer on alumina/ $\mathrm{NiAl}(110)$ taken as a function of the bias voltages. The monomer turns into a depression above $3.0 \mathrm{~V}$, as its negative excess charge triggers an upward bending of the alumina bands (see inset). The dimer appears bright due to tunneling through its LUMO located at $2.5 \mathrm{eV} .^{386}$ 


\section{Linear ad-chains}

The formation of anionic gold species on thin oxide films affects also their aggregation into larger particles at higher metal exposure, as demonstrated by the following STM experiments on $\mathrm{MgO}^{388,389}$ and alumina thin films. ${ }^{375}$ Experimentally, the aggregation of charged adspecies can be promoted by metal deposition at elevated temperature ( $>50 \mathrm{~K})$, where an enhanced atom mobility helps overcoming the inter-atomic Coulomb repulsion. In the initial growth stage (nominal coverage below $0.02 \mathrm{ML}$ ), Au clusters with distinct 1D shapes are frequently observed on 2-3 ML thick MgO/Ag(001) films (Fig. 4.13). This observation is in sharp contrast to the $\mathrm{Au}$ growth on bulk-like $\mathrm{MgO}$, where the formation of compact 3D clusters is strongly favored. ${ }^{37,390}$ The smallest Au aggregate detected on the MgO film has $9 \AA$ apparent length and $0.8 \AA$ height and is assigned to a flat-lying dimer. Five additional 1D clusters are identified, whose length increases in steps of $2.5 \AA$ from 12 to $22 \AA$. They are tentatively attributed to ad-chains containing three to seven Au atoms (Fig. 4.13). Most of the chains align with the two orthogonal MgO[100] directions, where $\mathrm{Mg}$ and $\mathrm{O}$ ions alternate in the surface, and only occasionally [110]-oriented chains are found.
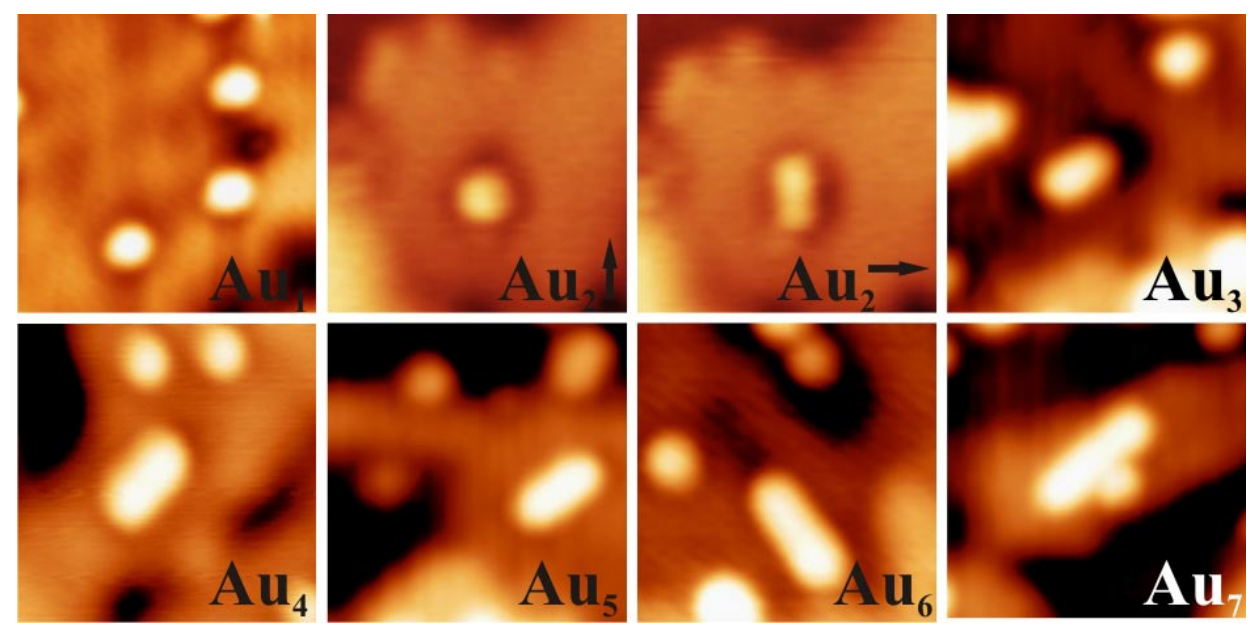

Fig. 4.13:

STM images of Au monomers, dimers and different chains on 2ML MgO/ $\mathrm{Ag}(001)(-0.4 \mathrm{~V}, 19 \times 19$ $\left.\mathrm{nm}^{2}\right)$. For the dimer, an upright $\left(\mathrm{Au}_{2} \uparrow\right)$ and a flat-lying isomer are observed $\left(\mathrm{Au}_{2} \rightarrow\right){ }^{389}$

At the first glance, the formation of 1D Au clusters appears surprising, as the number of stabilizing $\mathrm{Au}-\mathrm{Au}$ bonds is relatively small with respect to $2 \mathrm{D}$ and $3 \mathrm{D}$ aggregates. A linear atom arrangement is, however, consistent with the charged nature of the aggregates. In analogy to the discussion on $\mathrm{Au}$ monomers, the transfer of excess electrons into the affinity levels of $\mathrm{Au}$ clusters activates efficient electrostatic and polaronic binding schemes and increases their adsorption strength to the $\mathrm{MgO} / \mathrm{Ag}$ support. On the other hand, this chargemediated interaction gives rise to a substantial Coulomb repulsion between the additional 
electrons within the cluster. This electron-electron interaction can however be minimized when the excess charges are distributed over a long distance, which makes 1D shapes energetically favorable over 2D and 3D configurations. The observation of linear Au clusters on thin MgO films is therefore an experimental verification that the concept of charge-transfer prevails also for small aggregates on thin oxide films. ${ }^{388,389}$

This conclusion was corroborated with two DFT studies, calculating the stability of differently-shaped $\mathrm{Au}$ aggregates on $\mathrm{MgO} / \mathrm{Ag}(001)^{388}$ and $\mathrm{MgO} / \mathrm{Mo}(001)$ films. ${ }^{368}$ On the $\mathrm{MgO} / \mathrm{Ag}$ surface, the Au dimer as the smallest cluster occurs in two stable configurations, namely as an upright $\mathrm{Au}_{2}$ bound to an $\mathrm{O}^{2-}$ surface atom, and as a flat-lying species attached to two $\mathrm{Mg}^{2+}$ or two hollow sites in the oxide film. The upright dimer is neutral and has a closedshell electronic structure, reproducing the $\mathrm{Au}_{2}$ adsorption geometry on bulk MgO. ${ }^{316}$ The flatlying species, in contrast, is negatively charged (Bader charge $-0.8|\mathrm{e}|$ ) and binds $0.34 \mathrm{eV}$ stronger to the surface than its neutral counterpart (Fig. 4.14). This energetic preference already indicates the importance of charge-mediated binding mechanisms also for $\mathrm{Au}$ aggregates. Both, the flat and upright $\mathrm{Au}_{2}$ have been identified in the STM on the basis of their distinct height (0.8 $\AA$ versus $1.1 \AA$ ) and shape, demonstrating their coexistence on the surface despite of the different adsorption energy (Fig. 4.13). ${ }^{388}$

The thermodynamic stability of charged versus neutral ad-clusters becomes even larger for aggregates containing more than two atoms. ${ }^{389}$ According to the DFT calculations, a linear $\mathrm{Au}_{3}{ }^{-}$cluster (Bader charge $-1|\mathrm{e}|$ ) has by far the lowest energy, while other isomers, e.g. an upright or flat-lying triangle, spontaneously rearrange into the linear configuration during geometry optimization (Fig. 4.14). The same observation holds for the linear $\mathrm{Au}_{4}$ cluster that is $0.8 \mathrm{eV}$ lower in energy than a standing rhombus, being the equilibrium configuration on bulk $\mathrm{MgO}(001)$. The tetramer chain carries nearly two excess electrons (Bader value: -1.6|e|), which avoids the occurrence of an unpaired electron at $\mathrm{E}_{\mathrm{F}}$ and leads to a non-magnetic ground state of the system. The two extra electrons are mainly localized at the two terminal atoms of the chain, which minimizes the effect of the internal electron-electron repulsion and makes the linear atom assembly energetically favorable (Fig. 4.14). Also on the $\mathrm{MgO} / \mathrm{Mo}(001), \mathrm{Au}_{\mathrm{n}}$ clusters with $n=2-6$ were found to spontaneously charge up in order to maximize their interaction with the oxide surface. ${ }^{368}$

With increasing atom numbers, 2D Au aggregates gain more and more stability with respect to $1 \mathrm{D}$ configurations, as the creation of additional $\mathrm{Au}-\mathrm{Au}$ bonds outweighs the energy surplus from delocalizing the transfer electrons from the support. ${ }^{389}$ Whereas, the two-fold negatively 
charged $\mathrm{Au}_{5}{ }^{2-}$ chain on $\mathrm{MgO} / \mathrm{Ag}(001)$ is still iso-energetic with a flat-lying $\mathrm{Au}_{5}{ }^{-}$sheet, the $2 \mathrm{D}$ $\mathrm{Au}_{6}$ island is lower in energy than the corresponding chain by $0.2 \mathrm{eV}$ (Fig. 4.14). The energetic preference in this case is assigned to three additional $\mathrm{Au}-\mathrm{Au}$ bonds formed in the $\mathrm{Au}_{6}$ sheet, while the amount of charge transfer (-1.8|e| versus -1.6|e|) and the spatial delocalization of the extra electrons are comparable in the 1D and 2D isomers. The critical size of five to six Au atoms, at which the crossover between 1D and 2D growth occurs, has been verified by STM experiments on the MgO/Ag(001) film. Whereas three- and four-atom Au chains were frequently observed on the surface, only two $\mathrm{Au}_{6}$ and one $\mathrm{Au}_{7}$ chain could be identified along with a large number of similarly sized 2D isomers.

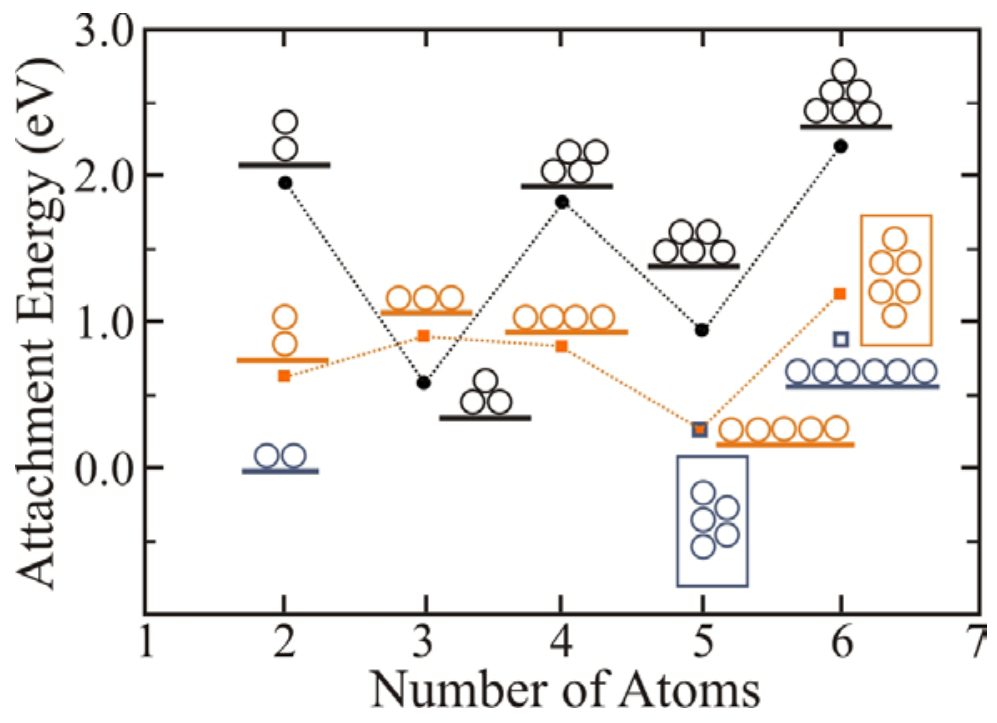

Fig. 4.14:

Attachment energies to form the different Au clusters shown in the insets, calculated for bulk $\mathrm{MgO}(001)$ (black circles) and for a $2 \mathrm{ML} \mathrm{MgO/Ag(001)} \mathrm{film} \mathrm{(orange} \mathrm{squares).} \mathrm{The} \mathrm{blue} \mathrm{squares} \mathrm{depict}$ the second most stable isomers on the thin film. On the oxide film, linear configurations are energetically preferred up to $\mathrm{Au}_{5}$, when planar isomers become more stable. The odd-even oscillations in the attachment energy are related to the high energy of open-shell systems containing an unpaired electron. They are less pronounced on thin $\mathrm{MgO}$ films due to the screening influence of the $\mathrm{Ag}$ support. $^{389}$

So far, the amount of charge transfer into oxide-supported Au clusters has been deduced only from DFT calculations. However at certain circumstances, this quantity becomes accessible to the experiment as well, namely when the electron filling of quantum well states in the charged aggregate can be determined and compared to the situation of a neutral species. In principle, electronic properties of metal aggregates and in particular the nature of their highest-occupied (HOMO) and lowest-unoccupied molecular orbital (LUMO) can be revealed from STM conductance spectroscopy. ${ }^{100,104}$ How this technique is exploited to quantify the amount of charge transfer through oxide thin films shall be demonstrated for Au clusters on alumina/ 
$\operatorname{NiAl(110)}$ films in the following. ${ }^{375,387}$ The system is ideally suited for this kind of measurements, because the Au clusters are strongly bound and can thus be explored in a large bias window ranging from -3.0 to $+3.0 \mathrm{~V}$.

Similar to the behavior on $\mathrm{MgO}$ thin films, Au aggregates into linear clusters on the alumina surface at low temperature $(\mathrm{T}<150 \mathrm{~K}){ }^{387}$ The ad-chains are between 8 and $22 \AA$ long, being compatible with a linear assembly of two to seven Au atoms, and align with the [001] direction of the NiAl support (Fig. 4.15). This unique chain direction is surprising as more orientations are expected from the hexagonal symmetry of the oxide film and the presence of two reflection domains tilted by $48^{\circ}$ against each other (see Fig. 3.18). ${ }^{48,155}$ Apparently, the chain orientation is dictated by the $\mathrm{NiAl}$ underneath, manifesting a substantial participation of the metal support to the alumina-gold interaction as discussed earlier in this chapter. The unique direction is explained by the fact that $\mathrm{Au}$ chains form preferentially on top of the [001]-oriented $\mathrm{Al}$ rows in the $\mathrm{NiAl}$ surface. Only in this configuration, the Au-induced rupture of an oxide $\mathrm{Al}_{\mathrm{s}}-\mathrm{O}_{\mathrm{i}}$ bond can be stabilized by re-bounding the $\mathrm{O}_{\mathrm{i}}$ to an $\mathrm{Al}$ atom in the metal and the chain becomes thermodynamically stable. ${ }^{375}$ In contrast to the $\mathrm{MgO}$ case, the formation of linear ad-chains is therefore not only charge-driven, but additionally enforced by the linear arrangement of suitable Au adsorption sites in the alumina/NiAl system. It should be noted that Pd deposition onto the same surface does not lead to the formation of ad-chains, and besides monomeric species only compact 3D clusters are observed. ${ }^{391}$ Apparently, the Pdalumina interaction is not governed by charging effects, in correspondence to the findings on the MgO thin film. ${ }^{374}$
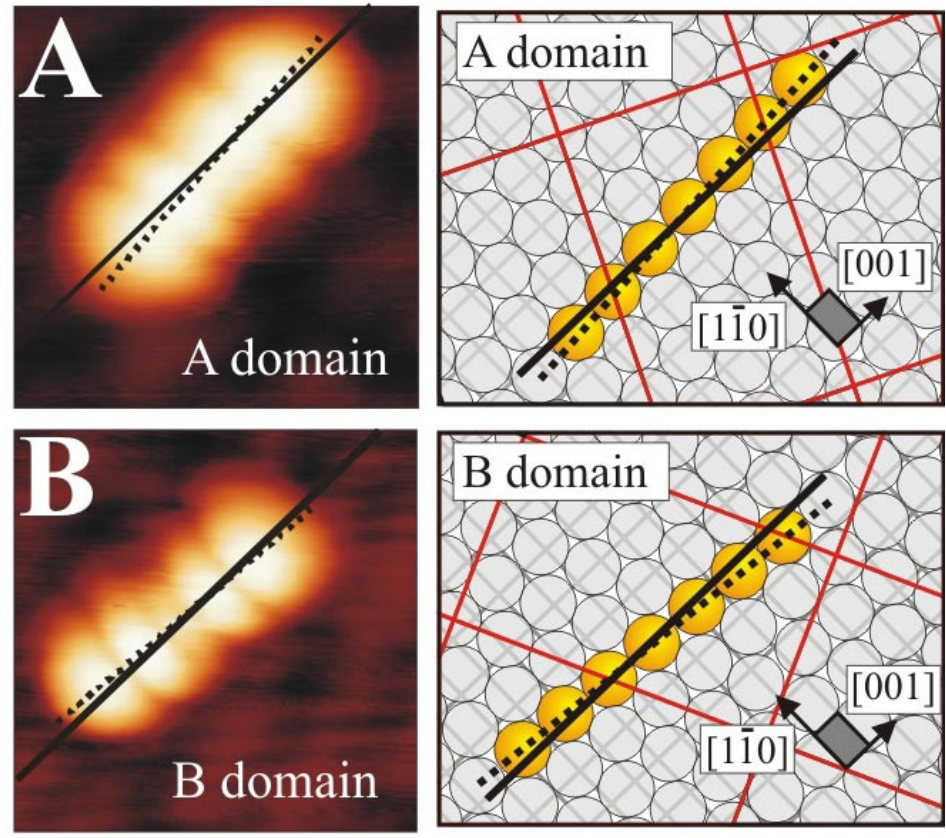

Fig. 4.15:

STM image and structure model for $\mathrm{Au}_{7}$ chains bound to the two reflection domains in the alumina/ $\mathrm{NiAl}(110)$ film $\left(5 \times 5 \mathrm{~nm}^{2}\right)$. The chain orientation and the NiAl [001] direction are indicated by dashed and solid lines, respectively. Although the two domains are tilted against each other by $48^{\circ}$, the chains closely follow the $\mathrm{NiAl}$ [001] direction in both cases. The sketches on the right side show the $\mathrm{Al}_{\mathrm{s}}^{3+}$ top layer (open balls), the Au chain atoms (yellow, filled balls), as well as the $\mathrm{NiAl}$ and alumina unit cells, being depicted with grey and red lines, respectively. ${ }^{387}$ 
The Au chains on the alumina thin film exhibit a particularly simple electronic structure around the Fermi level, which develops from the delocalization of the $s$ and $p$-like states in the 1D potential well. ${ }^{100,375}$ The resulting quantum well states (QWS) can be traced back to the hybridization between atomic orbitals of the adjacent chain atoms. According to the DFT, the HOMO of a single $\mathrm{Au}$ ad-atom derives from the $A u$ 6s orbital including a small $d$ contribution. It is localized at $-1.3 \mathrm{eV}$ below $\mathrm{E}_{\mathrm{F}}$ and hence doubly occupied (Fig. 4.16). As the $A u 6 s$ orbital is only half filled in the gas-phase species, the Au on the alumina film is negatively charged. The LUMO, on the other hand, is a $p_{z}$-like state located at $+2.5 \mathrm{eV}$ and therefore inside the alumina conduction band (calculated onset at $+1.8 \mathrm{eV}$ ). In the Au dimer, the two $6 s$-atomic orbitals hybridize and form new states at $-0.3 \mathrm{eV}$ and $-1.5 \mathrm{eV}$. Both states are filled, which brings the total number of s-electrons to four. Two of these valence electrons originate from the $\mathrm{NiAl}$ support, making the dimer two-fold negatively charged. The $\mathrm{Au}_{2}$ LUMO shifts to $+1.9 \mathrm{eV}$ due to the superposition of the two $p_{z}$ states of each monomer (Fig. 4.16). In the linear trimer, a third s-like state appears directly at the Fermi level, while the two other QWS shift to $-1.8 \mathrm{eV}$ and $-2.5 \mathrm{eV}$. Due to the half-filled nature of the HOMO, five $s$ electrons occupy the trimer valence states, three intrinsic ones from the $A u 6 s$ orbtials and two transfer electrons from the metal support. The unoccupied $\mathrm{Au}_{3}$ states comprise a symmetric and an anti-symmetric combination of the $p_{z}$ orbitals, giving rise to QWS at +1.9 and $+2.8 \mathrm{eV}$. It should be noted that calculated orbital energies will be systematically lower than the respective experimental results, due to the known underestimation of band gaps of insulators in the DFT.

The electronic fingerprints of Au monomer, dimer and trimer have, in parts, been reproduced by $\mathrm{dI} / \mathrm{dV}$ spectroscopy with the STM (Fig. 4.16, D-F) ${ }^{375}$ Whereas the monomer shows a similar conductance behavior as the bare alumina film, one $\mathrm{dI} / \mathrm{dV}$ maximum at $+2.6 \mathrm{~V}$ and two maxima at $+2.3 /+3.0 \mathrm{~V}$ have been detected for the dimer and trimer, respectively. These peaks at positive bias are compatible with the lowest $p_{z}$-like QWS in the dimer and the two lowest ones in the trimer. Conductance maps taken at the respective bias positions support this assignment. Whereas a homogeneous $\mathrm{dI} / \mathrm{dV}$ intensity distribution is revealed for the lower QWS, reflecting the constant density probability of the ground-state $p_{z}$ orbital, a region of suppressed conductance marks the nodal plane in the first excited $p_{z}$-derived state (Fig. 4.16 F). The invisibility of the LUMO in the $\mathrm{dI} / \mathrm{dV}$ spectra of the Au monomer is explained by its strong overlap with the alumina conduction band that dominates the conductance signal. No occupied QWS were detected for all three ad-species, reflecting the strong localization of the 
respective wave functions in the ultra-small Au aggregates and the generally low probability to extract electrons from filled adsobate levels in presence of an insulating film.

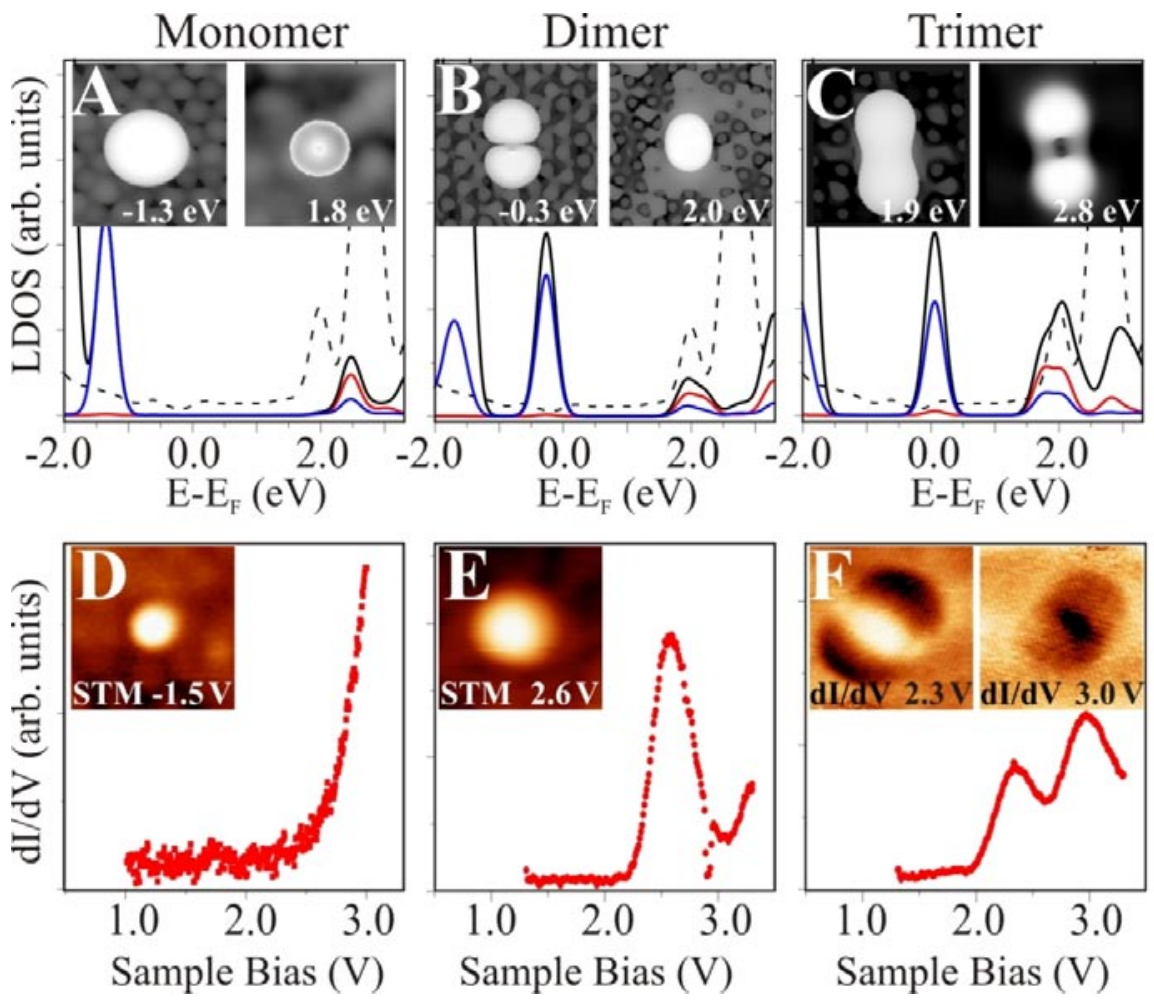

Fig. 4.16:

Spin-averaged LDOS and orbital shapes for (A) a Au monomer, (B) a dimer and (C) a trimer on alumina / $\mathrm{NiAl}(110)$, as calculated with DFT. The black line marks the total Au LDOS, the blue and red lines denote the $s$ - and $p_{z}$-contributions, respectively. The dashed line depicts the alumina states. (D,E) Experimental dI/dV spectra and topographic images of an Au monomer and a dimer. (F) Trimer spectrum with $\mathrm{dI} / \mathrm{dV}$ images taken at the two peak positions. All images are $4.5 \times 4.5 \mathrm{~nm}^{2}$ in size; the set-point for spectroscopy was set with $3.0 \mathrm{~V}$.

In analogy to the dimer and trimer, also the electronic structure of longer Au chains evolves from the consecutive splitting of the $s$ and $p$-like resonances of the chain atoms. As a result, a set of $m$ mainly occupied $s$-derived states and $m$ empty states with $p_{z}$-character develop in the valence region of an $m$-atom $\mathrm{Au}$ chain (Fig. 4.17). The s-like state manifold perfectly reproduces the behavior of a particle-in-a-box system with infinite walls. ${ }^{94}$ The eigenfunctions of the box potential are sinusoidals $\left(\psi_{n}=\sin k_{n} x\right)$, defined by a wave-number $k_{n}$ being proportional to the inverse box-length $L$ and a quantum number $n$ : $\left(k_{n}=n \pi / L\right)$. The resulting electron distribution in the $n^{\text {th }}$ QWS $\psi_{n}^{2}$ is therefore given by $n$ maxima separated by n-1 nodes along the well, while the eigen-energies follow a parabolic dispersion relation: ${ }^{94} E_{n}=E_{O}+\frac{\hbar^{2}}{2 m_{\text {eff }}}\left(\frac{\pi n}{L}\right)^{2}$. The experimental and theoretical properties of $s$-like QWS in the different Au chains are in good agreement with this particle-in-the-box picture, what the 
orbital shape and parabolic energy-dependence concerns (Fig. 4.17). Fitting the computed level energies to the predicted dispersion relation yields a potential depth $E_{0}$ of $-2.65 \mathrm{eV}$ and an effective electron mass $m_{\text {eff }}$ of $0.85 \mathrm{~m}_{\mathrm{e}}$ for electrons in the Au potential well. It is interesting to note that Au chains supported on a metallic $\mathrm{NiAl}(110)$ substrate show a smaller effective electron mass of $0.5 \mathrm{me}_{\mathrm{e}}{ }^{100,392}$ This finding indicates higher electron-mobility along the metalsupported chains, despite of a slightly larger Au-Au distance in this case (2.89 $\AA$ on $\mathrm{NiAl}$ versus $2.6 \AA$ on alumina). The difference is owed to the enhanced interaction between neighboring Au atoms on the metal surface, which is largely mediated by an indirect coupling via substrate electronic states and consequently missing on the insulating oxide film. ${ }^{107}$

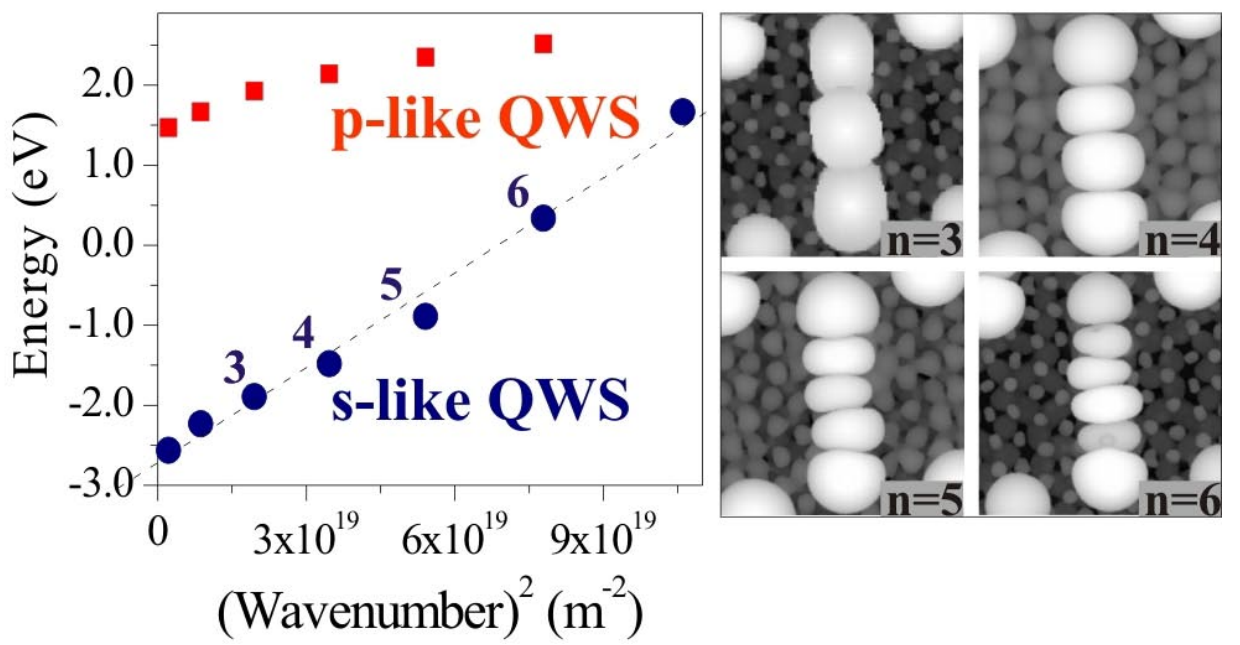

Fig. 4.17:

Dispersion relation for $s$ and $p$-like QWS in an $\mathrm{Au}_{7}$ chain on alumina/ NiAl(110). While the $s$-states show free-electron like behavior, the $p$-states have a non-parabolic dispersion relation. The computed shapes for the $3^{\text {rd }}$ to $6^{\text {th }} s$-like QWS are shown on the right. ${ }^{386}$

Also the STM conductance measurements on Au ad-chains of different length are in good agreement with the electronic structure sketched above and will be exploited to deduce the charge state of the respective ad-chains in the following. ${ }^{375,386}$ In contrast to ultra-small clusters, chains containing more than three atoms exhibit $\mathrm{dI} / \mathrm{dV}$ peaks at positive and negative sample bias, which means that electrons can be removed from filled QWS, too. The $\mathrm{Au}_{4}$ chain has two $\mathrm{dI} / \mathrm{dV}$ peaks below $\mathrm{E}_{\mathrm{F}}$, whereby the upper one at $-1.1 \mathrm{~V}$ (the HOMO) is characterized by three electron-density maxima and two nodal planes along the chain axis (Fig. 4.18). The HOMO of the tetramer chain therefore corresponds to the third s-like QWS. Assuming double occupancy of all QWS below the Fermi energy, the total number of s-electrons in the ad-chain will be six. Only four of these electrons originate from the initial $A u$ 6s orbitals of the four chain atoms, while two have been transferred from the NiAl support. With the same 
arguments, the $\mathrm{Au}_{5}$ chain turns out to be three-fold negatively charged, as its HOMO at $-1.6 \mathrm{~V}$ exhibits four intensity maxima and is thus assigned to the $4^{\text {th }}$ QWS. Only five of the eight $s$ electrons in these four levels have been donated from the Au atoms into the potential well, while the other three originate from the substrate. For the $\mathrm{Au}_{7}$ chain, being the longest one observed experimentally, the fourth and fifth QWS are detected at -1.8 and $-1.3 \mathrm{~V}$, respectively. In agreement with the particle-in-the-box model, the lower state shows four and the higher five density oscillations along the chain axis in the $\mathrm{dI} / \mathrm{dV}$ maps (Fig. 4.18). The $\mathrm{Au}_{7}$ chain has consequently five occupied QWS carrying ten s-electrons in total, which brings the number of transfer electrons from the NiAl to three again. It should be mentioned that no sixatom chains were experimentally identified. According to the DFT, the linear $\mathrm{Au}_{6}$ is three-fold negatively charged as the $\mathrm{Au}_{7}$ (nine s-electrons in total), but has a magnetic ground state due to the unpaired electron in its $\mathrm{HOMO}$ right at $\mathrm{E}_{\mathrm{F}}$. This unfavorable energetic situation might be responsible for the absence of $\mathrm{Au}_{6}$ chains in the experimental records. Six membered chains might either transform into a heptamer upon atom attachment or spontaneously rearrange into a planar 2D island, as earlier discussed for Au clusters on the $\mathrm{MgO} / \mathrm{Ag}(001)$ film. $^{389}$

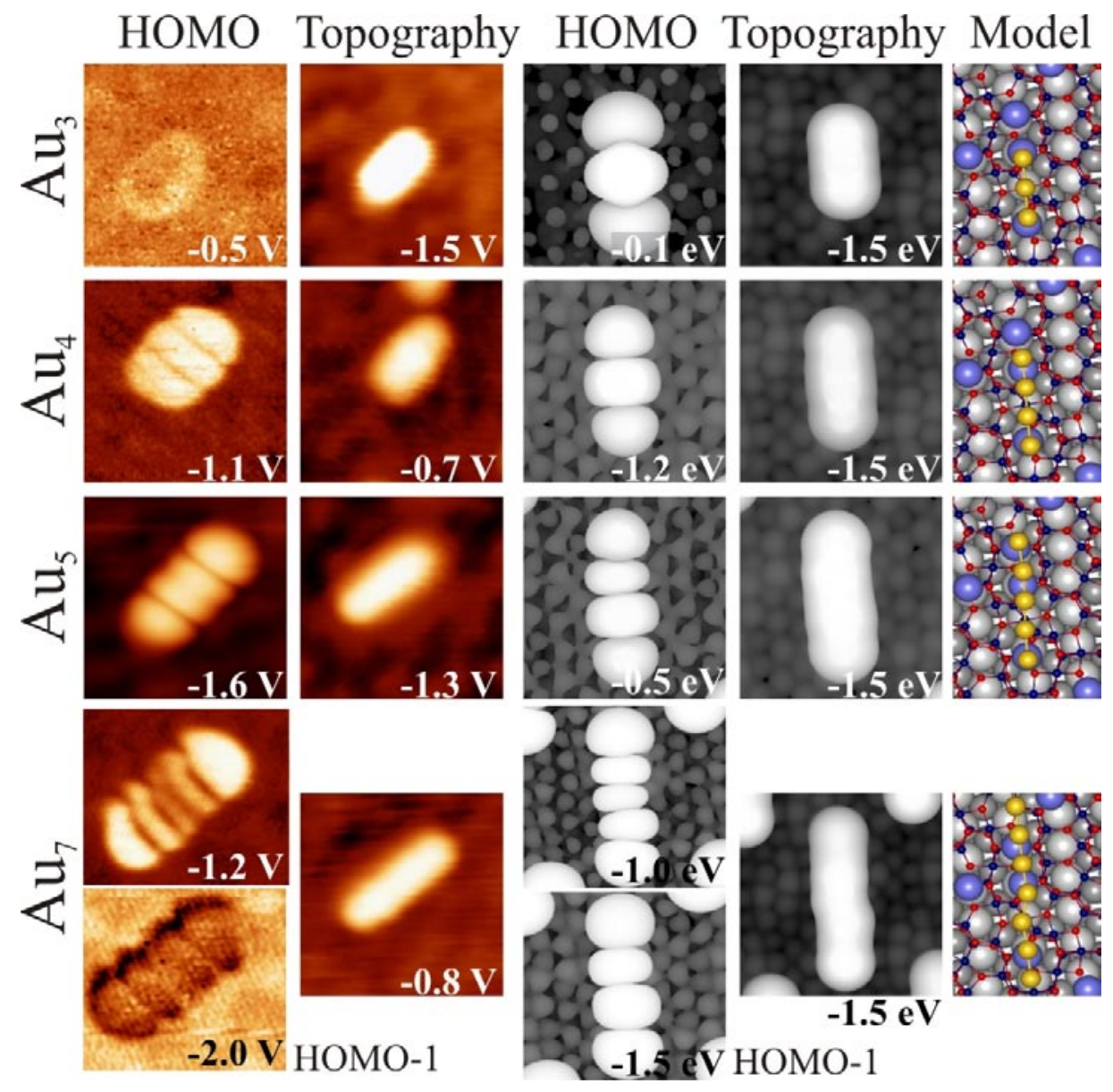

Fig. 4.18:

Experimental and calculated topography, HOMO shape and model structure for $\mathrm{Au}_{3}$ to $\mathrm{Au}_{7}$ chains on alumina $/ \mathrm{NiAl}(110)$. All images are $5.0 \times$ $5.0 \mathrm{~nm}^{2}$. The HOMO1 for the $\mathrm{Au}_{7}$ is shown in addition. Measured chain lengths are $9 \AA$, $12 \AA, 15 \AA$ and $22 \AA$; calculated distances between first and last chain atom amount to

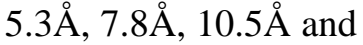
15.5A. To compare theoretical and exp. lengths, 2-3 $\AA$ should be added to both sides of the chain to account for the diffusivity of the $1 \mathrm{D}$ orbitals. ${ }^{375}$ 
The correspondence between the spectroscopic results and the calculated LDOS is less satisfying for the unoccupied state density of the Au ad-chains (not shown). Two dI/dV peaks are observed for the $\mathrm{Au}_{4}$ and $\mathrm{Au}_{5}$ and even three for the $\mathrm{Au}_{7}$ chains; however, the overall symmetry of the underlying orbitals is low and their nodal structure rather complex. ${ }^{387}$ On the other hand, also computed shapes for the unoccupied chain orbitals are heavily distorted with respect to the idealized $p_{z}$-QWS. Two effects have to be taken into account to rationalize the low symmetry of states above the Fermi level. First, the electron potential along the chain axis is highly inhomogeneous, because the chain atoms are subject to deviating binding contributions from the NiAl support and bind to 4 and 5-fold coordinated $\mathrm{Al}_{\mathrm{s}}^{3+}$ ions in the film. Apparently, the $p_{z}$-like states are more susceptible to these potential modulations than the s-states, reflecting the free-electron nature of the latter. And second, the unoccupied LDOS is not only governed by $p_{z}$-like QWS, but unoccupied $s$-like states emerge in this energy region as well. The interference of states with different symmetries adds to the complexity of the experimental and theoretical LDOS maps and renders an identification of the underlying sand $p$-like QWS difficult.

However, the observation of s-derived QWS below the Fermi energy is sufficient to determine the charge state of linear $\mathrm{Au}$ clusters on the alumina film with the STM. The approach discussed here therefore provides a purely experimental verification for the concept of charge transfer through thin oxide films and reproduces the theoretical predictions. ${ }^{186}$

\section{Two-dimensional islands}

The charge transfer from the metal substrate remains active for even larger Au clusters on oxide thin films and keeps controlling their geometric and electronic properties. ${ }^{376,377}$ On $2 \mathrm{ML}$ $\mathrm{MgO} / \mathrm{Ag}(001)$ films, Au grows in the form of flat, single-layer islands and develops a nearly complete wetting layer with increasing coverage (see Fig. 4.22 at the end of this chapter). ${ }^{393}$ The formation of 2D islands is in sharp contrast to the 3D growth regime that is generally observed for metals on bulk oxides. ${ }^{37,46,394}$ It reflects the tendency of Au to maximize the contact area with the oxide film and therewith the number of electrons being transferred into the Au affinity levels. Similar to the mechanism discussed for Au monomers and chains, charged gold islands show an increased adhesion to the oxide surface, resulting from polarization and electrostatic contributions to the total binding energy. ${ }^{376,377}$ For a closepacked $\mathrm{Au}$ layer on 2ML MgO/Ag(001), the average charge transfer per adatom has been calculated to $-0.2|\mathrm{e}|{ }^{395}$ 
Also for flat $\mathrm{Au}$ islands on $\mathrm{MgO} / \mathrm{Ag}(001)$, the charge state can be determined by means of conductance spectroscopy and imaging with the STM. The development of well-defined QWS forms again the necessary precondition for revealing this quantity, whereby the symmetry and electron filling of the QWS needs to be compared to the respective behavior in neutral aggregates. As electron confinement in a 2D potential is much more sensitive to structural irregularities, the $\mathrm{Au}$ islands under investigation need to be of rather high symmetry and free of defects. Those requirements are accomplishable when the gold is deposited onto the MgO film with small atom fluxes and at elevated temperature (50-100 K).

At idealized conditions, the QWS in nearly circular and flat Au islands resemble the eigenstates of a free electron-gas confined in a 2D parabolic potential (Fig. 4.19). ${ }^{376}$ The surprising simplicity of the electronic structure is owed to the dominance of $\mathrm{Au} 6$ s-derived states in the MgO band gap that extends from -4 to $+2.4 \mathrm{eV}$ in a $2 \mathrm{ML}$ thick oxide film. ${ }^{143}$ These $s$-like states do also not interfere with the $A u 5 d$ - and $6 p$-states at lower and higher energy, respectively. The 2D QWS are characterized by the number of nodes in their electron density probability, whereby states with S, P, D, F, G and H symmetry (defined by an angular momentum quantum number $m$ of 0 to 5 ) display 0-5 nodal planes, respectively (Fig. 4.19). 94,396 The QWS in a parabolic potential follow a linear dispersion relation, according to $E_{n, m} \propto$ $E_{0}(2 n-1+m)$ with $n$ the orbital quantum number. The states are $2 \times(n+m)$-times degenerated in the $(n+m)^{\text {th }}$ electronic shell and shell closing therefore occurs for 2, 6, 12, 20 and 30 electrons in the potential well.

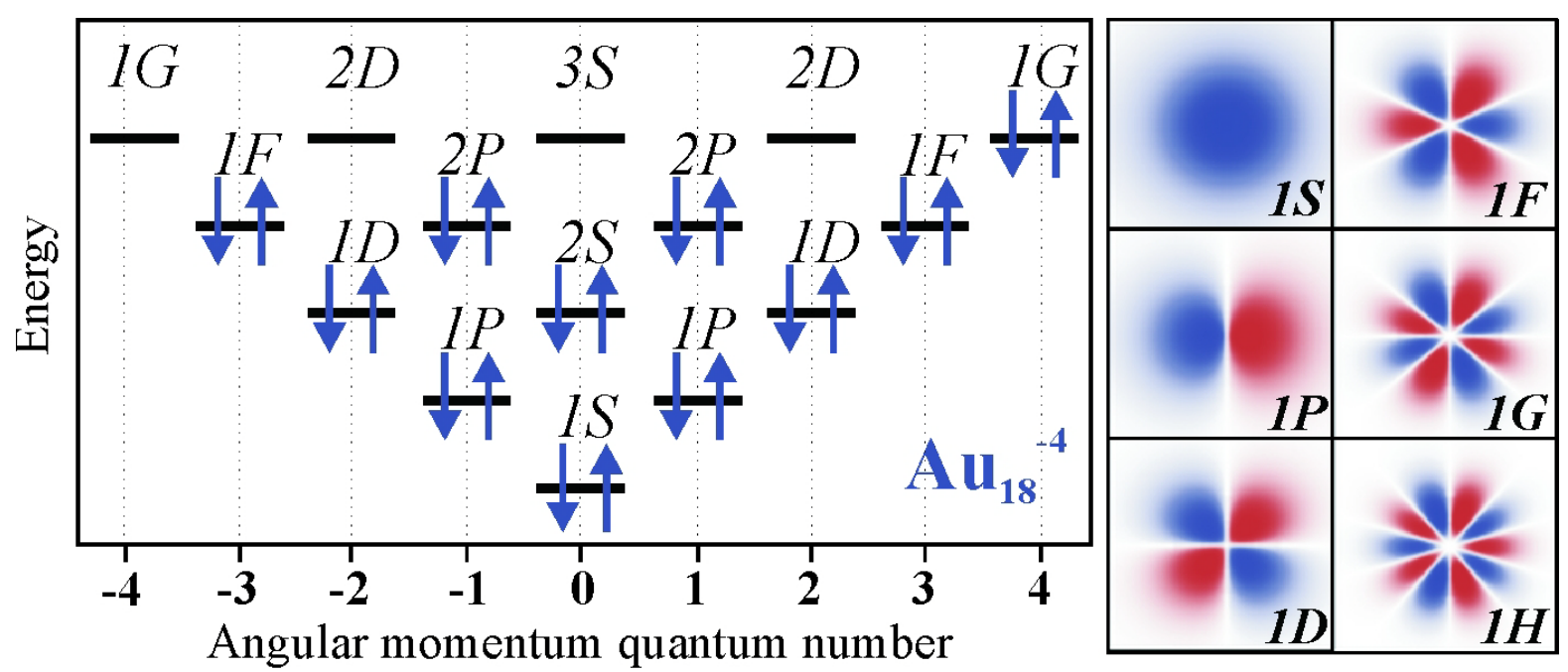

Fig. 4.19:

Energy levels (left) and orbital shapes (right) of the free-electron eigenstates in a 2D parabolic potential. The electron occupancy determined for a particular Au cluster on 2ML MgO/Ag(001) (here the $\mathrm{Au}_{18}$ cluster) is depicted by arrows. 
Such free-electron-like QWS are indeed found to govern the electronic structure of ultra-small Au clusters deposited on 2ML MgO/Ag(001). ${ }^{395}$ In low-bias STM measurements, the clusters exhibit compact shapes of approximately 10-20 $\AA$ diameter and 0.8-0.9 $\AA$ height, which reflects their approximate topographic structure (Fig. 4.20A). With increasing bias, flowerlike protrusions emerge in the STM images and the apparent cluster height increases by nearly $50 \%$. The change in appearance indicates that the electronic properties of the cluster are now responsible for the imaging contrast. ${ }^{68}$ More precisely, a single QWS that dominates the electron transport at the selected bias voltage dictates the new cluster shape in the STM. In most examples, the underlying orbital is characterized by an irregular lobe pattern, manifesting the disordered arrangement of $\mathrm{Au}$ atoms within the aggregate. However, also highly symmetric orbitals are occasionally observed, which reproduce the characteristic shape of free-electron-like QWS in a 2D parabolic potential in almost perfect manner (Fig. 4.20).
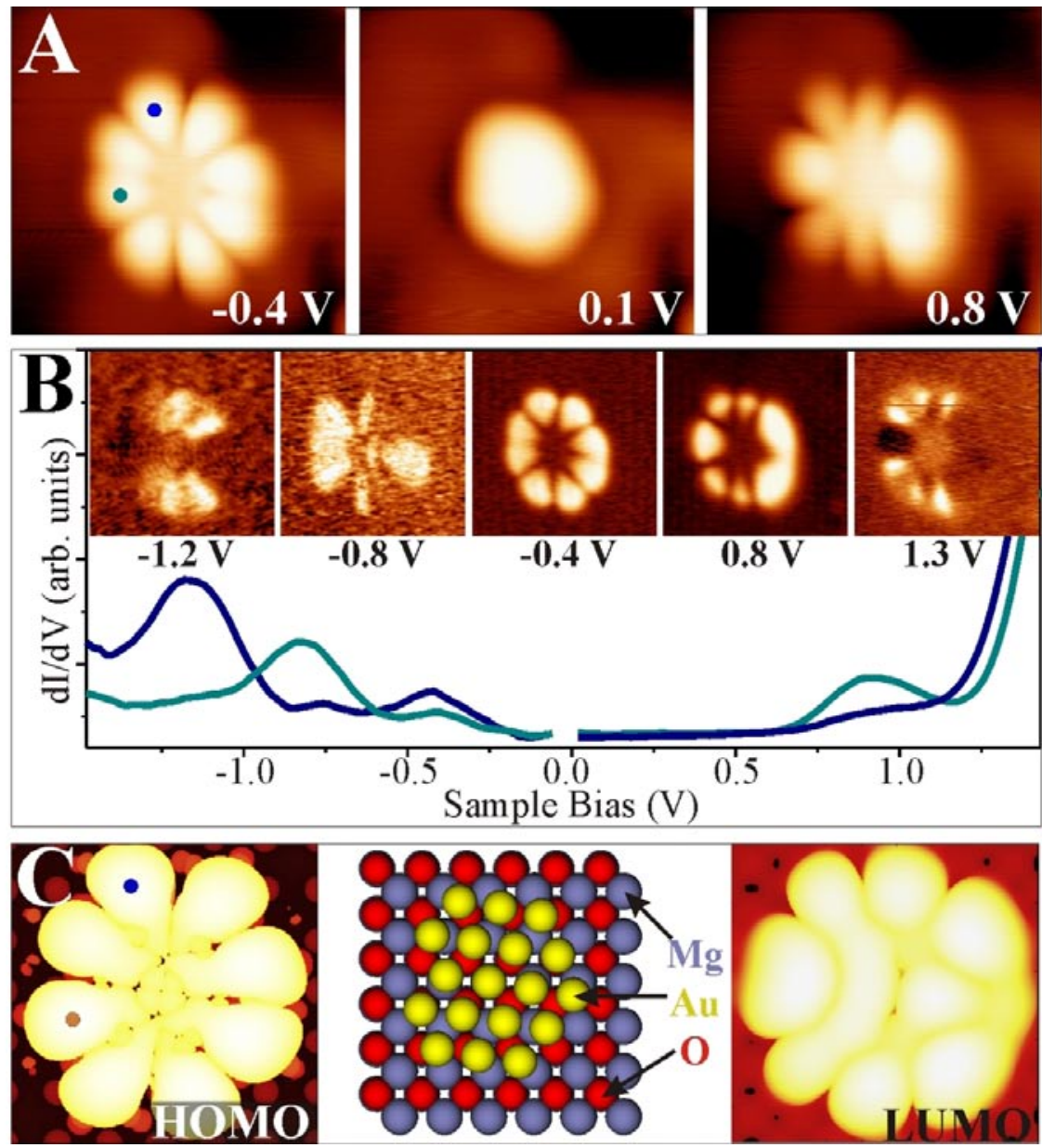

Fig. 4.20:

(A) Topographic and (B) dI/dV images of a symmetric Au cluster on $2 \mathrm{ML} \mathrm{MgO/} \mathrm{Ag}(001)$ taken at the given sample bias $\left(3.9 \times 3.9 \mathrm{~nm}^{2}\right)$. The corresponding $\mathrm{dI} / \mathrm{dV}$ spectra are shown in addition (blue and cyan curves: top and left part of the cluster). (C) Calculated HOMO and LUMO shape, as well as structure model of an $\mathrm{Au}_{18}$ cluster on $\mathrm{MgO} / \mathrm{Ag}(001)$. Perfect match between experimental and theoretical cluster properties indicate the identity of both aggregates. ${ }^{395}$ 
Conductance spectroscopy reveals the energy position of the respective electronic states. For the Au cluster shown in Fig. 4.20, the HOMO and LUMO are clearly identified as dI/dV peaks at -0.4 and $+0.8 \mathrm{~V}$, respectively, separated by a region of zero-conductance of roughly 1.0 V width. The shape of both, the HOMO and LUMO, is characterized by four nodal planes, indicating $G$-symmetry of the underlying orbitals. Also higher QWS show up in the $\mathrm{dI} / \mathrm{dV}$ spectra of the cluster. The HOMO-1 at $-0.8 \mathrm{~V}$ and the HOMO-2 at $-1.2 \mathrm{~V}$ are both of $P$ symmetry with the nodal plane being orthogonal in the two cases. The shape of the HOMO-1 is less defined and interferes with the HOMO symmetry, as tunneling into the latter still controls the integral conductance of the junction and therewith the tip-sample distance. As a result, artificial maxima appear in the $\mathrm{dI} / \mathrm{dV}$ maps of the HOMO-1 at the node positions of the HOMO. The same effect is observed for the LUMO+1, where extra $\mathrm{dI} / \mathrm{dV}$ maxima become visible at the LUMO nodes. In all images, small differences between the left and right half of the cluster are apparent, suggesting a slight asymmetry in its atomic configuration.

Based on the STM data, the structural and electronic properties of the Au cluster and therefore its charge state can be determined by comparing the experimental orbital shapes with DFT calculations of different sample clusters. For this purpose, an extensive structural search for monolayer Au clusters with matching properties has been conducted. ${ }^{376,395}$ The experimental signature of the cluster in Fig. 4.20 is perfectly reproduced by a planar $\mathrm{Au}_{18}$ cluster, whose structure is derived from a highly-symmetric $\mathrm{Au}_{19}$ cluster with one corner atom being removed. In accordance to the experiment, its HOMO and LUMO are the two $1 G$ orbitals located in the $5^{\text {th }}$ shell of the harmonic potential, whereas the HOMO- 1 and HOMO-2 are the $2 P$-like QWS states in the $4^{\text {th }}$ electronic shell (see level scheme in Fig. 4.19). The missing atom with respect to a 'magic' $\mathrm{Au}_{19}$ clusters introduces the slight asymmetry in the orbital shapes, which becomes particularly evident for the LUMO. The asymmetry is also responsible for the occurrence of a HOMO-LUMO gap, being in contrast to the identical $1 G$ level energies predicted for the harmonic potential. The $\mathrm{Au}_{18}$ cluster has in total eleven occupied valence orbitals and therefore 22 s-electrons (see Fig. 4.19). Each of the $18 \mathrm{Au}$ atoms donates its 6s electron to the delocalized QWS, which implies that the missing four electrons have been introduced via charge transfer from the $\mathrm{MgO} / \mathrm{Ag}$ interface. The presence of four excess charges on the $\mathrm{Au}_{18}$ cluster is corroborated by a Bader analysis that yields a value of $-3.54|\mathrm{e}|$. This result is also in good agreement with the average transfer of $0.2|\mathrm{e}|$ per atom calculated for a dense-packed Au layer on the $\mathrm{MgO}$ thin film. ${ }^{395}$ 
Similar procedures have been carried out for several other Au clusters on $\mathrm{MgO} / \mathrm{Ag}(001)$, containing between 8 and 20 atoms. ${ }^{395}$ In all cases, a negative charging of the ad-cluster was revealed, verifying the concept of a charge-mediated binding of $\mathrm{Au}$ islands to oxide thin films. ${ }^{186,368,374}$ The number of transfer electrons was found to be proportional to the atom number in the 2D cluster or, synonymic, to the interfacial contact area (Fig. 4.21B). Interestingly, the accumulated charge per atom in 2D aggregates is hereby lower than in $1 \mathrm{D}$ chains for small atom counts, manifesting the higher efficiency of linear structures in distributing the excess electrons and lowering the internal Coulomb repulsion. ${ }^{375,389}$ This energetic preference diminishes, however, for Au clusters containing more than 6-7 atoms and triggers the crossover from $1 \mathrm{D}$ to $2 \mathrm{D}$ growth as discussed before.
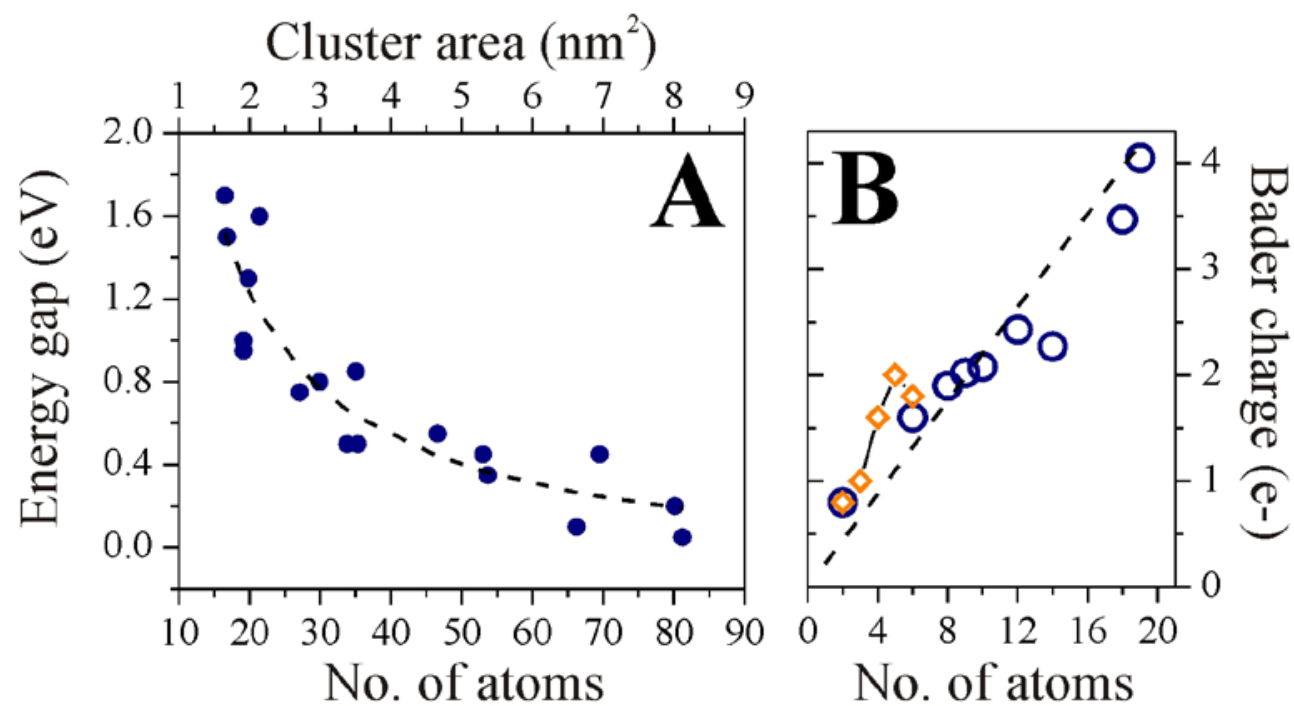

Fig. 4.21: (A) Experimental HOMO-LUMO gap for differently sized Au clusters on $\mathrm{MgO} / \mathrm{Ag}(001)$. The atom number is deduced from the measured particle area $\Omega$, using DFT results as a reference for the de-convolution procedure. The dashed line is a fit of the data to the inverse cluster size. (B) Calculated number of excess electrons in linear (diamonds) ${ }^{389}$ and 2D Au clusters on 2ML $\mathrm{MgO} / \mathrm{Ag}(001){ }^{395}$ The line depicts the charge accumulation of $0.2|\mathrm{e}|$ per atom, being computed for a compact Au layer on $2 \mathrm{ML} \mathrm{MgO} / \mathrm{Ag}(001)$.

It should be mentioned that also the HOMO-LUMO gap shows a strong dependence on the atom number per cluster (Fig. 4.21A). Whereas Au aggregates containing 10-15 atoms have experimental gap sizes around $1.5 \mathrm{eV}$, the HOMO-LUMO gap closes for more than 80 atoms in the cluster, reproducing earlier results obtained for $\mathrm{Au}$ clusters on $\mathrm{TiO}_{2}{ }^{333}$ The gap size hereby follows the inverse cluster area $\Omega$ according to $E_{g} \propto \Omega^{-1}$, as expected for the energy separation between the eigenstates in a 2D harmonic potential. ${ }^{20,396}$ 
The fact that a charge-induced interaction mechanism depends on the possibility to exchange electrons through the oxide layer has been exploited to modify the growth regime of $\mathrm{Au}$ on thin oxide films. ${ }^{377,393}$ When electron transfer through the oxide spacer is prohibited, the formation of charged 2D islands will be replaced by the 3D growth of neutral clusters, as observed for bulk oxides. ${ }^{394}$ The dominant transport mechanism for electrons through the oxide film is tunneling from the Fermi level of the metal support, ${ }^{186,368}$ as already proposed by Cabrera and Mott. ${ }^{373}$ The tunneling probability depends exponentially on the oxide thickness and to a smaller extent on the size of the band gap and the chemical nature of the metal-oxide interface. In the limit of thick oxide barriers, true electron exchange is replaced by the polarization of charges towards the Au ad-layer. ${ }^{397}$

The effect of oxide thickness on the Au growth behavior has first been analyzed with DFT calculations. $^{377}$ The study revealed a 50\% decrease in the charge accumulation in planar $\mathrm{Au}_{8}$ clusters when the MgO thickness increased from two to five layers. As a result of the inhibited charge transfer, the adhesion of the $\mathrm{Au}_{8}$ cluster reduced from 0.7 to $0.35 \mathrm{eV}$ per interfacial $\mathrm{Au}$ atom and the formation of compact 3D aggregates with a high number of internal $\mathrm{Au}-\mathrm{Au}$ bonds became energetically favorable. This crossover from 2D to $3 \mathrm{D}$ growth regime with increasing oxide thickness was experimentally verified later for the $\mathrm{MgO} / \mathrm{Ag}(001)$ system (Fig. 4.22). ${ }^{393}$ Whereas only single-layer islands were found upon Au deposition onto a three ML MgO film, particles with pronounced 3D shapes emerged on an eight layer thick film. This experiment clearly demonstrated the possibility to control the dominant growth regime on thin-film systems by inhibiting the charge transfer through the insulating oxide spacer.
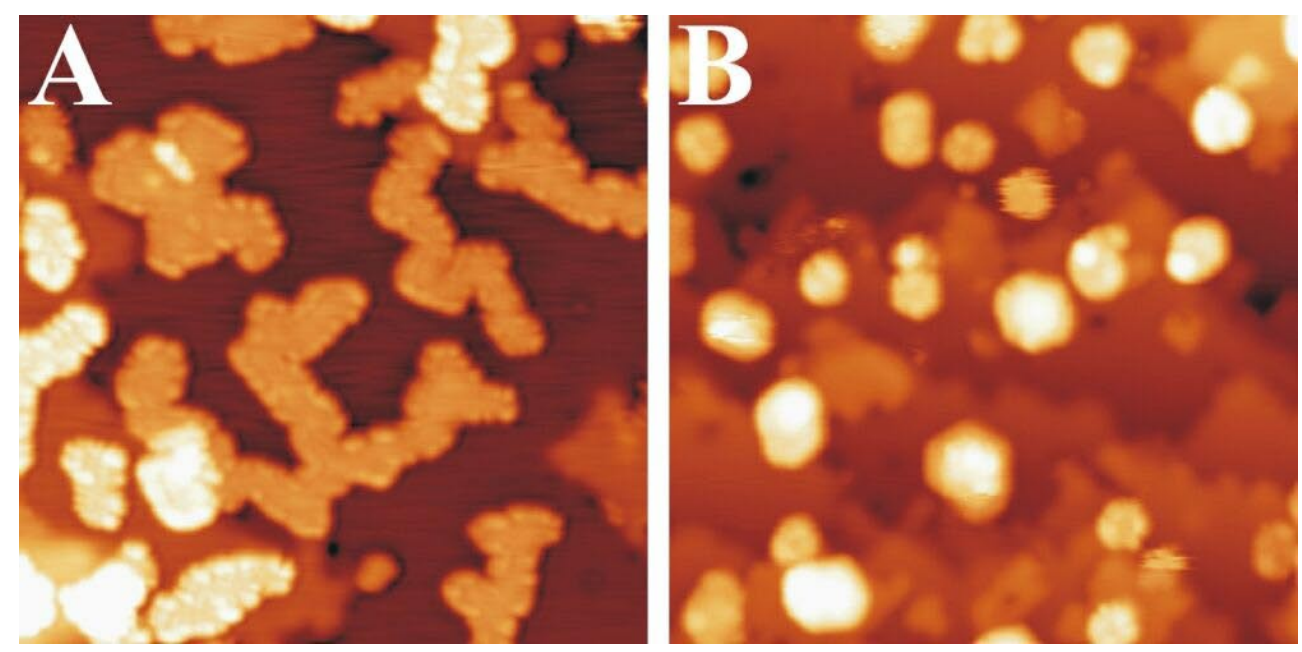

Fig. 4.22:

STM images of Au deposits on (A) $3 \mathrm{ML}$ and (B) $8 \mathrm{ML} \mathrm{MgO/Ag} \mathrm{(001)}\left(30 \times 30 \mathrm{~nm}^{2}\right)$. Due to the charged-mediated adhesion, Au wets the ultra-thin $\mathrm{MgO}$ film. In the case of thicker films, the electron transfer is inhibited and 3D gold particles form on the oxide surface. ${ }^{393}$ 


\section{Molecular charging}

The formation of charged adsorbates on ultra-thin oxide films is not limited to metal adstructures, but has been observed for molecular species, too. ${ }^{84,398,399}$ In contrast to metal clusters, complex organic molecules have another degree of freedom that interferes with the charging behavior, namely their structural conformation. The occurrence of charge transfer is therefore not only governed by the energy position of the molecular affinity levels with respect to the Fermi energy, but depends additionally on the actual binding geometry of the molecule and its coupling strength to the support. Consequently, different charge states can be stabilized for chemically identical molecules on thin oxide films and even converted into one another; opening the possibility to produce charge-sensitive molecular switches on oxide supports.

First experimental evidence for the presence of charged molecules has been obtained for copper phthalocyanine (CuPc) deposited at $12 \mathrm{~K}$ on alumina thin films on $\mathrm{NiAl}(110){ }^{84,399} \mathrm{In}$ the unbiased STM junction, the molecule adsorbs in the neutral charge state on the surface. It is imaged as a small entity with cross-like shape at low sample bias, indicating the absence of molecular orbitals for electron transport around $\mathrm{E}_{\mathrm{F}}{ }^{103}$ The appearance of the molecule suddenly changes in a bias range between 0.35 and $0.75 \mathrm{~V}$, when the eight-lobe symmetry of the molecular LUMO becomes visible in the STM (Fig. 4.23). Surprisingly, the same orbital structure reappears in images taken at negative bias $(-0.5 \mathrm{~V})$, suggesting that the LUMO dominates the STM topographic contrast again. This observation is explained by the response of the alumina film and the molecular orbitals to the tip-induced electric field, which induces a down-shift of the states at increasingly negative bias. The magnitude of this shift depends on the thickness and the dielectric constant of the alumina film, as discussed in detail in Chapter 2.1. At a certain bias threshold, the LUMO drops below the NiAl Fermi level and fills up with electrons, causing the molecule to charge up negatively. In this case, the junction conductance is again controlled by the hence occupied LUMO and its characteristic shape reappears in the STM images (bipolar tunneling). ${ }^{83}$ The charging bias can be deduced from a sharp peak at roughly $-0.4 \mathrm{~V}$ in the $\mathrm{dI} / \mathrm{dV}$ spectra of $\mathrm{CuPc}$, which marks the sudden filling of the LUMO by electron transfer from the NiAl support (Fig. 4.23A). Due to a different response of the central and outer parts of the CuPc to the tip electric-field, the charging threshold slightly varies across the molecule, and switching of its charge state can even be observed upon scanning the species at fixed bias (Fig. 4.23B). ${ }^{399}$ 

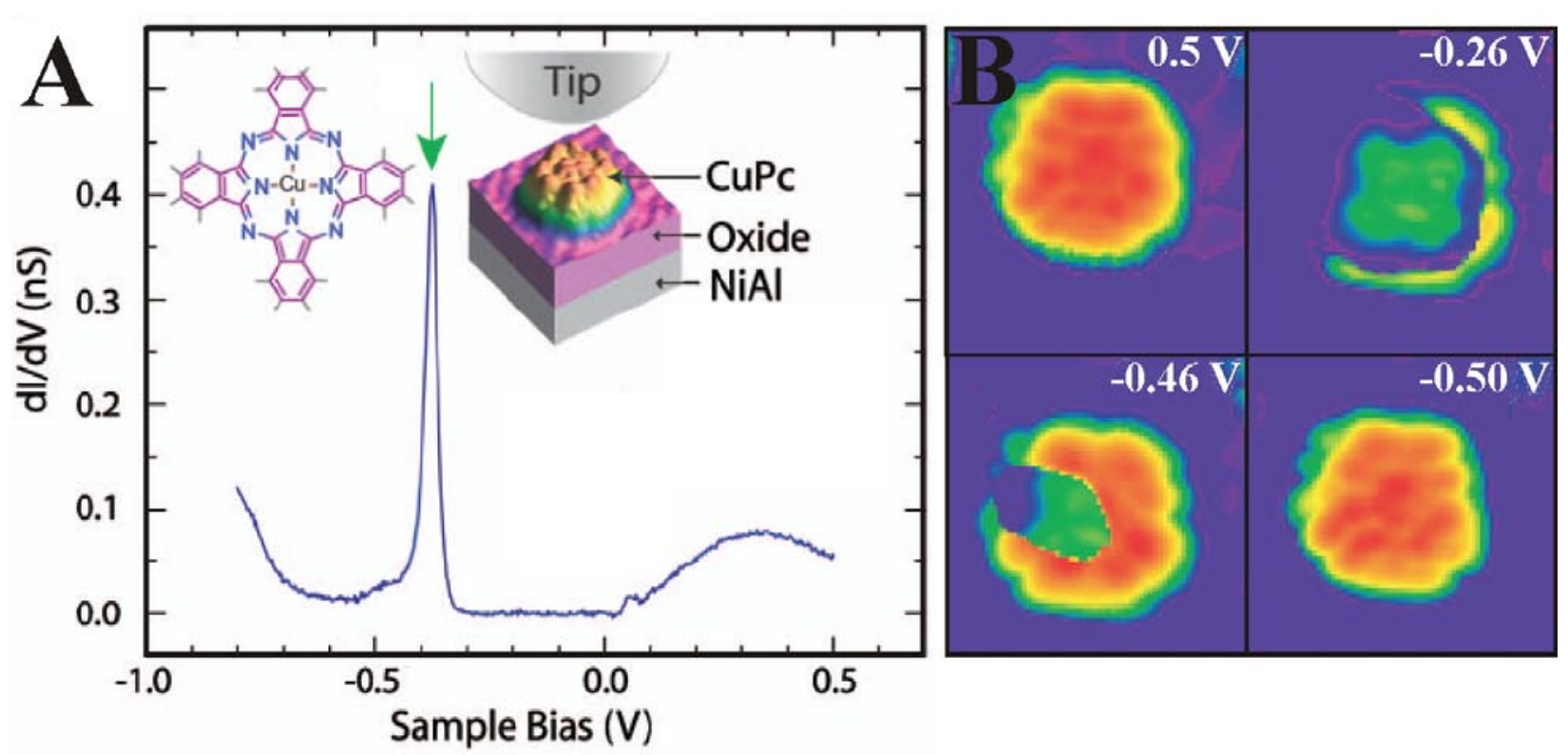

Fig. 4.23: (A) $\mathrm{dI} / \mathrm{dV}$ spectrum of a CuPc molecule on alumina/NiAl(110). The broad peak at positive bias corresponds to the molecular LUMO, whereas the sharp peak at $-0.4 \mathrm{~V}$ marks the charging of $\mathrm{CuPc}$ via electron transfer from the NiAl support. (B) STM topographic images of CuPc molecules take at different bias values $\left(4 \times 4 \mathrm{~nm}^{2}\right)$. At +0.5 and $-0.58 \mathrm{~V}$, the either empty or filled LUMO determines the image contrast. The discontinuous appearance of the molecule at -0.26 and $-0.46 \mathrm{~V}$ reflects sudden changes of its charge state when tunneling out of different molecular regions. The green cross visible in the center of the $-0.26 \mathrm{~V}$ scan reflects the shape of the neutral species. ${ }^{399}$

Whereas the different charge states of the CuPc molecule are only stabilized by the tipinduced electric field, a true charge bi-stability is detected for magnesium porphyrin (MgP) molecules on alumina/NiAl(110). ${ }^{398}$ The as-adsorbed molecule is neutral again. However, increasing the sample bias above $+0.85 \mathrm{~V}$ induces a negative charging of the molecule, which becomes manifest by a sudden drop of the tunnel current and the differential conductance in the respective I-V curve (Fig. 4.24). In contrast to the CuPc, negative charging is activated at positive sample bias and follows a different mechanism. The decisive step is the temporal filling of the $\alpha$-LUMO with electrons from the tip, stimulating its splitting into two singlyoccupied molecular orbitals (a SUMO and a SOMO). Whereas the lower one drops below the Fermi level and takes one electron from the support, the upper one, being offset in energy by the intra-molecular Coulomb energy $U$, remains empty (Fig. 4.24). The change of the charge state is accompanied by a conformational modification of the molecule that in turn stabilizes the extra electron even after the sample bias has been switched off. The molecular charging is only reversed by lowering the applied voltage to $-1.2 \mathrm{~V}$, when tunneling out of the occupied SOMO sets in. Upon removing its electron, the charge-induced electronic and structural conformation of the MgP collapses and the molecule returns to its neutral ground state. ${ }^{398}$ The alumina film plays an important role in this charging sequence, as it substantially reduces the tunneling probability between molecule and metal support. By this means, the life-time of the 
hot electron in the initially empty $\alpha$-LUMO (or of the hot hole in the SOMO) is sufficiently prolonged to trigger the molecular switching. In a subsequent experiment on the same $\mathrm{MgP} / a$ lumina/NiAl system, it could be shown that the charge-mediated switching hysteresis of the molecule can even be initiated by photon irradiation to produce the hot electron in the $\alpha$-LUMO. ${ }^{400}$ In this case, the STM tip is only required to probe the charge state of the molecule and to restore its neutral configuration by removing the electron from the filled SOMO.

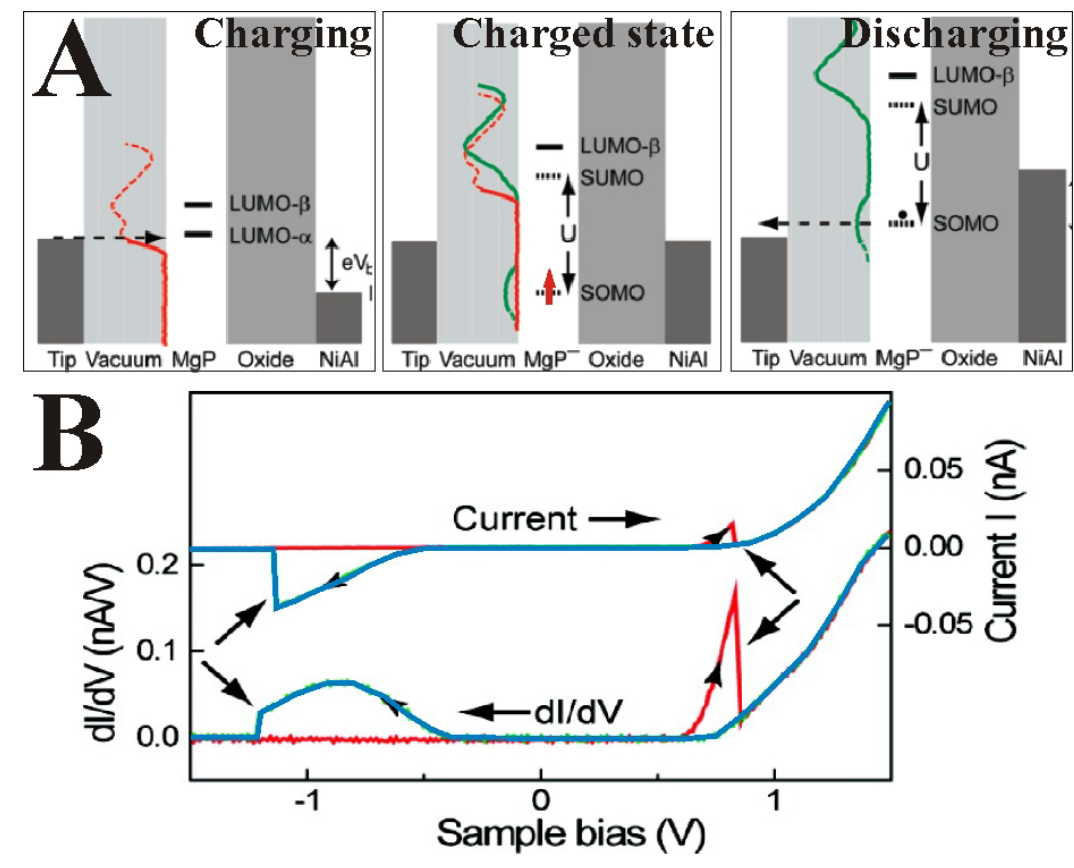

\section{Fig. 4.24:}

(A) Energy diagram showing the electron injecting into the $\alpha$ LUMO of a neutral MgP molecule on alumina/NiAl(110) (left). In the charged molecule, a filled SOMO and an empty SUMO are present, separated by the Coulomb energy $U$ (middle). Discharging occurs by releasing the electron from the SOMO at negative bias (right). (B) $I-V$ (top) and $\mathrm{d} I / \mathrm{d} V$ (bottom) spectra measured on a MgP molecule on alumina/NiAl(110). The sudden changes in the current and $\mathrm{d} I / d V$ signal depict charging and discharging events at positive and negative sample bias, respectively. ${ }^{398}$

The previous chapter has demonstrated that the adsorption of atoms, molecules and metal clusters on thin oxide films on metal supports is often accompanied by the transfer of electrons through the oxide spacer. Driving force for the charge exchange is a modification of the vacuum energy at the oxide surface, either induced by an electric dipole at the metal-oxide interface or by an external electric field. As a consequence, low lying affinity levels of the adsorbate might fall below the Fermi energy of the metal-oxide system and fill up with electrons, as observed for Au species on MgO and alumina thin films. In rare cases, also the reverse effect is observed, where initially occupied adsorbate levels shift above $E_{F}$, resulting in the formation of cationic surface species. An example for this behavior is the Au adsorption on $\mathrm{FeO} / \mathrm{Pt}(111)$ and will be discussed in the following paragraph. ${ }^{372}$ In general, charged adspecies show an enhanced adhesion to the oxide thin films due to presence of additional charge-mediated binding mechanisms, e.g. electrostatic or polaronic interactions. 
The dependence of charge-driven interaction schemes on the nature and thickness of the oxide spacer as well as on the chemical identity of the ad-species opens a number of possibilities to modify the adsorption characteristic of thin-film systems. ${ }^{53,367}$ Not only the charge state of single adsorbates can be controlled in this way, but also the growth and wetting behavior of metal-oxide systems becomes adjustable to a certain extent. Furthermore, adsorbate systems with a charge-bistability might be fabricated, having potential applications as charge-sensitive switches to be used in molecular electronics. ${ }^{401}$ The investigation on how the adsorption properties of oxide thin films might be influenced in a desired way is just at the beginning, and additional examples for such attempts can be found in the following chapters.

\subsubsection{Adsorption on polar oxide films}

Also in thin-film systems presented in the following, charge-mediated binding mechanisms might occur, however, they are covered by other, more pronounced effects. This paragraph discusses the peculiarities of atomic and molecular adsorption on polar oxide surfaces. As demonstrated in Chapter $\underline{3.6}$, uncompensated oxide polarity leads to a diverging electric dipole with increasing layer thickness, rendering those systems thermodynamically unstable. $^{33,271}$ The polarity might be healed be removing the associated excess charges, for instance via restructuring of the top-most oxide layers or surface metallization. The most frequently observed mechanism for polarity healing is, however, the attachment of charged or strongly polarizable adsorbates, which makes adsorption experiments on polar oxide surfaces particularly interesting. Unfortunately, the number of such experiments performed with the STM is surprisingly small. Most of them deal with polar $\mathrm{ZnO}(0001)$ surfaces, ${ }^{361,363}$ or explore the hydroxylation of thin oxide films with polar character, for instance of $\mathrm{Cr}_{2} \mathrm{O}_{3} / \mathrm{Cr}(110)^{402}$ or more recently of FeO/Pt(111). ${ }^{303}$ For the latter system, also experiments on other atomic and molecular adsorbates have been performed with low-temperature STM, which shall be presented in the following. ${ }^{168,403,404}$

The surface polarity of the $\mathrm{FeO}$ film arises from the stacking of a negatively-charged $\mathrm{O}^{\delta-}$ surface layer above a positively-charged $\mathrm{Fe}^{\delta+}$ interface layer, separated by $0.68 \AA$ mean distance (see Chapter $\underline{3.6}$ ). ${ }^{172,295}$ The FeO film forms a coincidence structure with the $\mathrm{Pt}$ support, in which the Fe atoms alternately occupy top (O on fcc), fcc (O on hcp) and hcp sites (O on top) in the $\mathrm{Pt}(111)$ surface, according to the model shown in Fig. $3.23 .{ }^{190}$ As a consequence, the Fe-O layer separation is not constant across the coincidence cell, but varies between $0.78 \AA$ in fcc and hcp domains and $0.52 \AA$ in the top region. ${ }^{166}$ This distance 
modulation affects the strength of the local dipole in the oxide surface, which is largest for the hcp and smallest for the top domain according to surface-potential maps obtained with the STM. ${ }^{190}$ These periodic changes in the surface polarity are now expected to govern the spatial distribution of adsorbates on the $\mathrm{FeO}$ surface.

The first demonstration of this effect came from low-temperature STM experiments dealing with the adsorption of single $\mathrm{Au}$ atoms on the polar film. ${ }^{403}$ The adatoms, being imaged as circular protrusions of almost $10 \AA$ diameter, show a large tendency to attach to the hcp domains of the coincidence cell (Fig. 4.25A). At a nominal Au coverage of 0.01 ML, being close to one atom per coincidence unit cell, more than $70 \%$ of the hcp domains are occupied with $\mathrm{Au}$ atoms, whereas adjacent fcc and top regions remain nearly adsorbate-free (occupancy smaller than 10\%). This site-specific Au adsorption behavior leads to the formation of a hexagonal adatom array, whose lattice constant of $25 \AA$ matches the size of the FeO/Pt(111) superstructure.
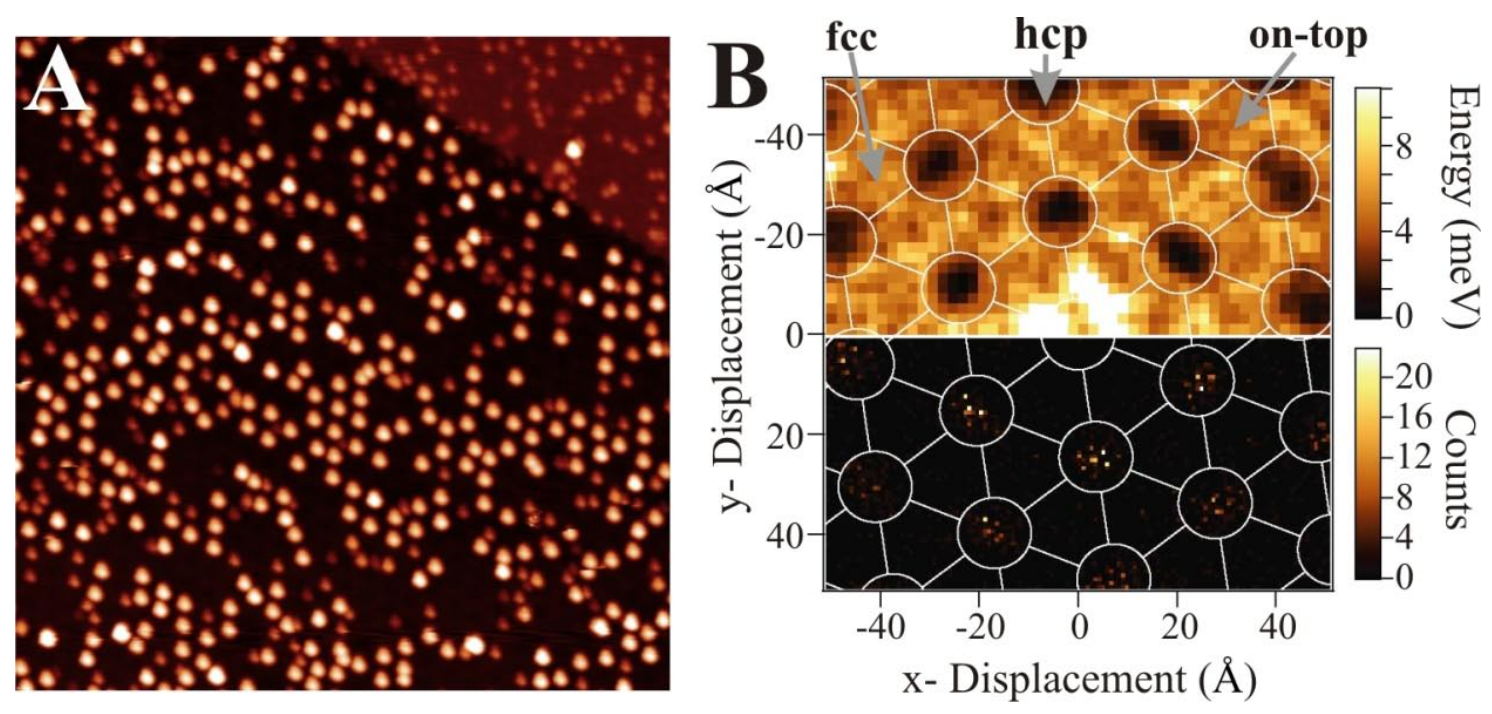

Fig. 4.25: (A) STM topographic image of Au atoms on FeO/Pt(111) $\left(0.5 \mathrm{~V}, 60 \times 60 \mathrm{~nm}^{2}\right)$. (B) Twodimensional pair-distribution function of the adatoms on the FeO film (lower part). Most events occur in the hcp domains; the sharp maxima within those regions reflect the atomic binding sites of the adatoms. The upper part depicts the potential landscape for Au adsorption, being calculated by assuming a Boltzmann distribution of the Au binding energies. ${ }^{403}$

The long-range order in the adatom arrangement becomes evident in the 2D pair-distribution function $n(x, y)$ calculated from approximately 700 adatom positions on the FeO film (Fig. 4.25B). The highest probability to find a neighboring $\mathrm{Au}$ atom is along the high-symmetry axes of the FeO/Pt system in $25 \AA$ distance from the origin. Due to the perfect ordering, even $2^{\text {nd }}$ and $3^{\text {rd }}$ neighbor positions in the adatom lattice show substantial occupancy. Each maximum in the pair distribution function is split into 6-7 spots forming a hexagonal pattern 
with $3.0 \AA$ lattice constant. This fine-structure represents the few atomic binding sites in the centre of the hcp domains that are actually populated by Au. Based on the pair distribution function, the modulation in the Au binding energy $\Delta E$ across the coincidence cell is estimated by assuming a Boltzmann distribution for the occupation probability: $n(x, y) \propto \exp ($ $\Delta E /(k T){ }^{405,406}$ As the self-assembly is observed for temperatures as high as $50 \mathrm{~K}$, this value is used to calculate the lower bound for the energy modulation $\triangle E$. The analysis yields an energetic preference of $(10 \pm 2) \mathrm{meV}$ for Au binding to the hcp domains with respect to the adjacent fcc and top regions. It is interesting to note that Au atoms remain essentially monomeric during self-assembly even at relatively high coverage. No aggregation sets in before the majority of hcp domains is populated, suggesting a repulsive, most likely Coulombtype interaction between the adatoms that retards the cluster formation.

The self-assembly of $\mathrm{Au}$ atoms on the $\mathrm{FeO} / \mathrm{Pt}(111)$ surface can directly be related to the polar nature of the oxide film. ${ }^{403}$ The adatoms preferentially attach to the hcp domains that have been identified as the ones with the highest surface polarity. ${ }^{190}$ The strength of the uncompensated dipole in those regions enables an efficient polarization of the $\mathrm{Au}$, giving rise to an electrostatic interaction between the adatoms and the dipole field of the polar oxide film. This binding contribution will be smaller on the adjacent fcc and top domains due to the reduced dipole strength in these regions. Furthermore, the enlarged Fe-O layer distance in hcp domains might facilitate the formation of true chemical bonds with the Au atom, further enhancing the binding preference to this domain type.

A detailed insight into the Au-FeO binding mechanism is revealed from DFT calculations. ${ }^{372}$ Analyzing the Au interaction with O-top, Fe-top, bridge and hollow site in all three regions of the coincidence cell yields the highest binding energy for the O-top sites in the hcp domains. The calculated binding strength for the adatom is with $0.6 \mathrm{eV}$ almost $30 \%$ higher than on Fehcp sites, but also 30\% higher than on O-sites in the fcc and top domains, rationalizing the observed site-specific adsorption behavior of gold. An even stronger adsorption was predicted for an inverted geometry, where a Fe atom flips above the O-plane to coordinate the Au (see Fig. 4.9). This binding configuration was, however, not approved by the STM experiments. Apparently, the energy barrier to swap the local Fe-O stacking sequence is not overcome at the low temperature of the experiment.

The Au binding to an O-site in the hcp domain leads to an increase of the local Fe-O layer distance by almost 20\%, which further enlarges the surface dipole and initiates a substantial strengthening of the Au-FeO bond. Such polaronic lattice distortion is stimulated by a positive 
charging of the $\mathrm{Au}$ atom, which lifts the $\mathrm{O}$ ion underneath above the surface plane and slightly repels the three neighboring Fe ions. ${ }^{370,372}$ The charged character of the Au is supported by the calculated state density for the hcp-bound adatom (Fig. 4.26). The Au 6s orbital in this plot is located at $+0.3 \mathrm{eV}$ above the Fermi level and empty, in contrast to its half-filled nature in the gas-phase Au atom. This finding suggests that the $6 \mathrm{~s}$ electron has been transferred into the support, which is the reverse effect than observed for $\mathrm{Au}$ atoms on $\mathrm{MgO}$ and alumina thin films that charge up negatively. ${ }^{186,375}$ The formation of cationic gold on FeO/Pt(111) is triggered by the unusually high work-function of the polar oxide system that impedes charge transfer out of the support. ${ }^{166,372}$ Furthermore, attachment of a cationic adsorbate can be considered as the most efficient way to reduce the uncompensated oxide dipole, lowering the surface-free-energy of the polar film. ${ }^{271}$ The adsorption of positively charged Au onto the FeO hcp-domain therefore shares similarities with the common polarity healing mechanism via hydroxylation ( $\mathrm{H}^{+}$attachment).
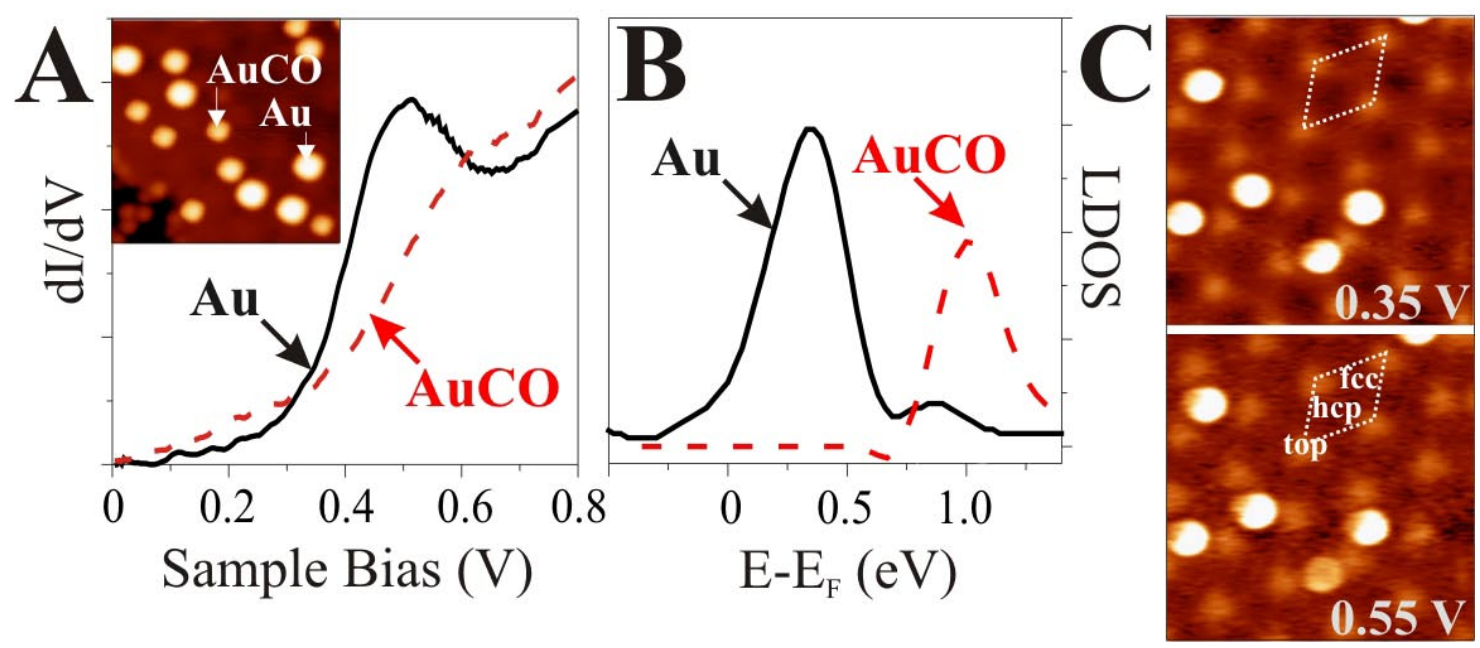

Fig. 4.26: (A) dI/dV spectra and (B) calculated LDOS of Au and AuCO species adsorbed on a hcpdomain of $\mathrm{FeO} / \mathrm{Pt}(111)$. The corresponding STM image is shown in the inset. The Au-related peak at $0.5 \mathrm{~V}$ (exp.) and $0.3 \mathrm{eV}$ (theory) corresponds to the $\mathrm{Au} 6 \mathrm{~s}$ orbital, while the AuCO state at $1.0 \mathrm{eV}$ is of CO $2 \pi^{*}$ character and not detected in the STM. ${ }^{372,}$ (C) dI/dV maps of differently-bound Au atoms (13 $\times 13 \mathrm{~nm}^{2}$ ). Whereas the upper four adatoms are attached to hcp domains and appear bright in conductance maps taken at the $A u 6 s$ resonance position $(0.55 \mathrm{~V})$, the lower species sits in another site and shows no 6s-related $\mathrm{d} / \mathrm{dV}$ intensity (lower panel). Below the resonance position, all adatoms appear with the same dI/dV contrast (upper panel). ${ }^{403}$

The unoccupied nature of the $A u$ ss orbital and the derived positive charge state of the adatom has been corroborated by conductance measurements on the FeO/Pt(111) system. Hcp-bound $\mathrm{Au}$ atoms exhibit a pronounced $\mathrm{dI} / \mathrm{dV}$ peak at $+0.5 \mathrm{~V}$ above the Fermi level, being assigned to the unfilled $\mathrm{Au}$ 6s orbital on the basis of the DFT results (Fig. 4.26A). ${ }^{372,403}$ Further evidence 
for the $6 s$ character of the state comes from CO adsorption onto the adatoms, which suppresses the $\mathrm{dI} / \mathrm{dV}$ peak at $+0.5 \mathrm{~V}$. In fact, the $A u$ s level shifts to higher energies upon $\mathrm{Au}-\mathrm{CO}$ bond formation due to the Pauli repulsion exerted by the CO molecular orbitals. ${ }^{407}$ However, even bare Au atoms do not display the $+0.5 \mathrm{~V} \mathrm{dI} / \mathrm{dV}$ peak if they are located on fcc or top domains of the coincidence cell, as demonstrated by the conductance maps shown in Fig. 4.27C. Apparently, those adatoms exhibit a deviating electronic structure and are not charged, which gives an additional explanation for their reduced adsorption energy with respect to the hcp-bound species. The self-assembly of Au atoms is therefore a direct consequence of the spatially modulated surface polarity in the FeO thin film, which enables positive charging and subsequent binding enhancement only for Au atoms bound to the hcp domains of the $\mathrm{FeO} / \mathrm{Pt}(111)$ system.
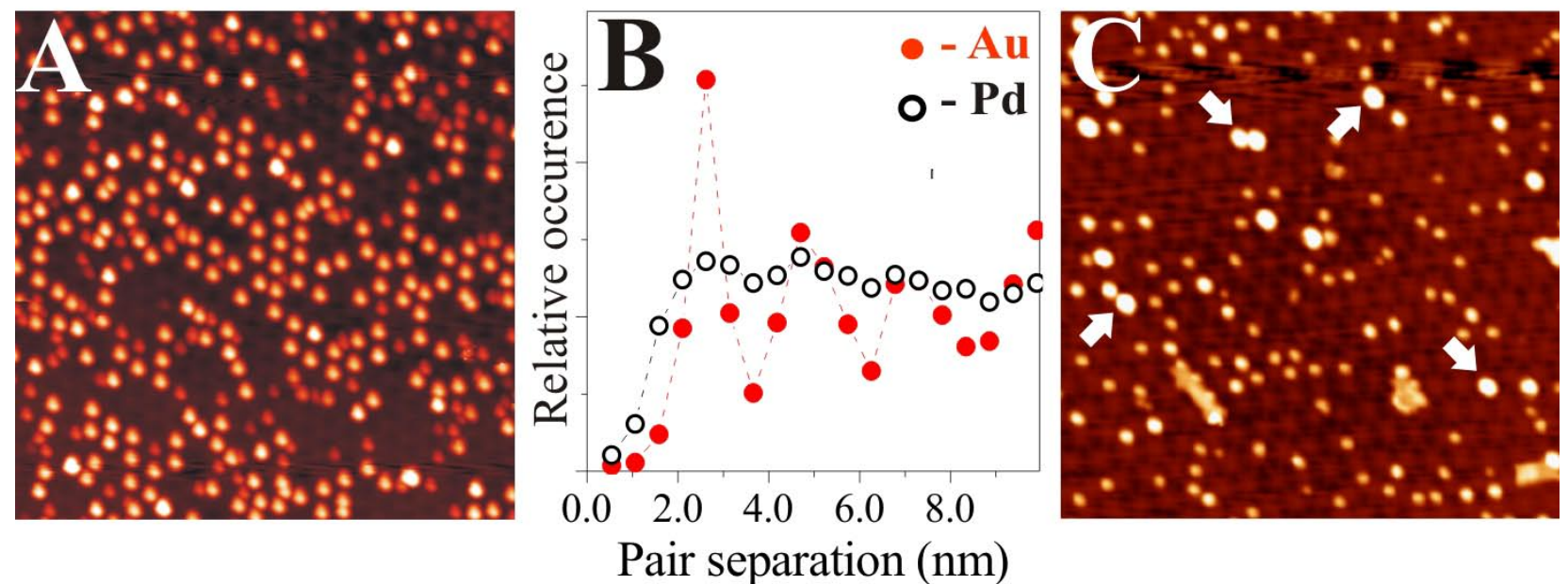

Pair separation (nm)

Fig. 4.27: STM images of (A) Au and (C) Pd atoms on FeO/Pt(111) $\left(0.25 \mathrm{~V}, 50 \times 50 \mathrm{~nm}^{2}\right)$. Arrows in (C) mark small Pd clusters. (B) Radial pair-distribution function for the Au and Pd adatoms on the FeO surface, calculated from hundreds of atom positions taken from different STM images. In contrast to $\mathrm{Au}$, no ordering effect is observed for the Pd atoms. ${ }^{372}$

As the binding behavior of Au is, to a certain extent, charge-mediated, it is not surprising that atoms with different electronic structure do not show the same ordering effect on the $\mathrm{FeO}$ film. In contrast to gold, Pd deposition at $10 \mathrm{~K}$ results in a random distribution of the adatoms on the oxide surface, as visualized with STM imaging and the derived radial pair-distribution function (Fig. 4.27). Furthermore, Pd shows a large tendency to form aggregates even at small metal exposure and low deposition temperature (see arrows in Fig. 4.27C). The absence of self-ordering effects for Pd is corroborated by the DFT calculations. ${ }^{372}$ The Pd atoms experience a much smoother adsorption landscape, where variations in the binding energy are only on the order of $\pm 100 \mathrm{meV}$ for the different sites of the FeO coincidence cell. On the other 
hand, the average binding strength for Pd is with $\mathrm{E}_{\mathrm{b}}=1.4 \mathrm{eV}$ much higher than for Au (0.6 $\mathrm{eV}$ ), which reduces the transient mobility of the adatoms after impinging the surface. Finally, no charge transfer is induced upon adsorption, because the $P d 5 s$ and the $P d 4 d$ orbitals are well above and below the Fermi level, respectively. The Pd atoms therefore remain in a neutral charge state in all considered binding positions of the FeO coincidence cell, which inhibits their ordered arrangement and facilitates an aggregation into clusters on the $\mathrm{FeO}$ surface.

A charging of adsorbates is, however, not essential to induce their self-organization on the polar FeO film. Especially in the absence of strong chemical forces, the modulated surfacedipole across the $\mathrm{FeO}$ coincidence cell might sufficiently perturb the potential landscape for adsorption to trigger ordering effects. Self-assembly phenomena that are exclusively driven by the spatially-varying oxide polarity are therefore expected for closed-shell, but highly polarizable electronic systems that are unable to form strong covalent bonds with the support. Ideal candidates in this respect are molecules with a conjugated $\pi$-electronic system that couple to the inert oxide surface mainly via polarization interactions. ${ }^{408}$ Therefore, magnesium phthalocyanine (MgPc) molecules have been chosen to study the adsorption of a polarizable, but chemically inactive molecular species on $\mathrm{FeO} / \mathrm{Pt}(111){ }^{404}$ Figure 4.28 shows an STM image taken after MgPc deposition onto the surface at $300 \mathrm{~K}$. Closer inspections of such measurements reveal that also the MgPc preferentially adsorbs in the hcp domains of the coincidence structure, in particular when those regions are adjacent to a rising step edge. Based on statistically relevant data, the fraction of molecules attached to the hcp domains was determined with $84 \%$, which compares to $14 \%$ and $2 \%$ bound to top and fcc regions, respectively. Assuming a Boltzmann distribution of the MgPc according to their binding energy, adsorption to the FeO hcp domain is preferred by $90 \mathrm{meV}$ over the fcc and by $46 \mathrm{meV}$ over the top region. This result clearly demonstrates the importance of polarization interactions of the MgPc in the dipolar field of the FeO. It should be noted that an electrostatic coupling between the $\mathrm{MgPc}$ and the $\mathrm{FeO}$ surface dipole requires the induction of a dipole moment in the originally planar molecule, for instance via a small vertical displacement of the central $\mathrm{Mg}^{\delta+}$ ion against the Pc-cage. ${ }^{409}$ 
The energetic preference for binding to the $\mathrm{FeO}$ hcp domains is almost one order of magnitude larger than for $\mathrm{Au}$ adatoms, ${ }^{403}$ which reflects the superior polarizability of the organic molecule. Furthermore, whereas polarization in the spatially modulated dipole field is the decisive interaction mechanism for the MgPc, this contribution competes with true chemical bonding for $\mathrm{Au}$ atoms. Because the latter effect is less site-specific, the spatial modulations in the Au binding energy are weaker and well-defined Au super-lattices only form at cryogenic temperatures. In contrast, the self-assembly of the MgPc is observed even at $300 \mathrm{~K}$ deposition temperature.
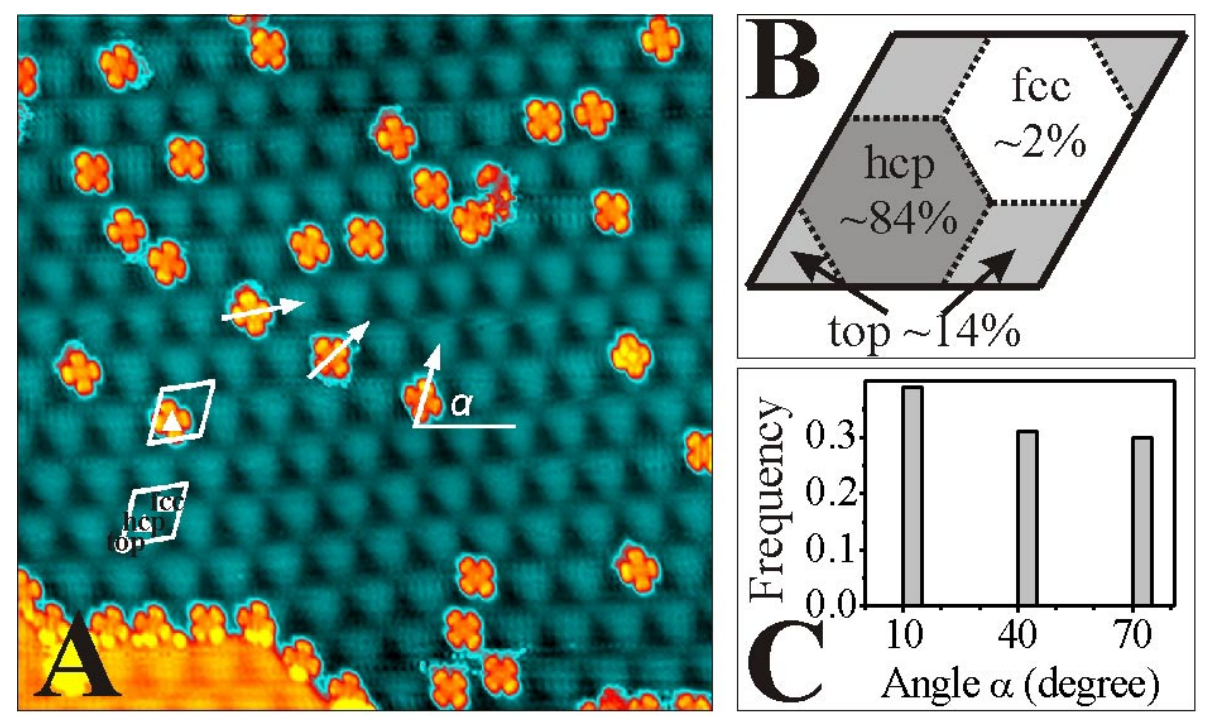

Fig. 4.28: (A) Topographic image of MgPc molecules on FeO/Pt(111) adsorbed at $300 \mathrm{~K}(35 \times 35$ $\mathrm{nm}^{2}$ ). The coincidence cell is indicated by the white parallelogram and the arrows mark the three rotational orientations of the molecules. (B) Occurrence of the MgPc in different domains of the $\mathrm{FeO}$ coincidence lattice. (C) Tilt angles of the MgPc symmetry axes with respect to a horizontal line. More than hundred molecules have been used for the statistical analysis in (B) and (C).

The ordering of the MgPc on the FeO thin film affects not only the spatial distribution, but also the rotational alignment of the molecule (Fig. 4.28C). The angular orientation is hereby independent of the FeO-Pt coincidence lattice that develops in different domains in the $\mathrm{FeO} / \mathrm{Pt}(111)$ system. $^{42}$ In fact, it is only determined by the local MgPc interaction with the hexagonal FeO lattice, which has essentially the same orientation throughout the surface and determines the three rotational alignments of the molecule. ${ }^{404}$ The self-ordering of the MgPc on $\mathrm{FeO} / \mathrm{Pt}(111)$ has therefore two aspects: While the spatial distribution is governed by the modulated surface potential within the coincidence cell, the angular alignment is fixed by the interaction of the molecule with the atomic FeO lattice. In general, the MgPc adsorption on polar $\mathrm{FeO}$ is a rare example, where self-assembly is not induced by inter-molecular coupling, ${ }^{410}$ but by a template effect of the oxide support. 
In summary, the fundamental adsorption properties of polar oxide surfaces have been discussed for atomic and molecular adsorbates on the FeO/Pt(111) film. In both examples, the uncompensated surface dipole of the oxide film influences considerably the binding behavior, predominately by inducing polarization and electrostatic interactions with the ad-species. The binding occurs preferentially in regions with large dipole strength, triggering the formation of long-range ordered adsorbate structures on the oxide surface. A similar behavior has been observed for $\mathrm{Cr}$ and $\mathrm{Fe}$ aggregates on polar $\mathrm{Fe}_{3} \mathrm{O}_{4}$ films grown on $\mathrm{Pt}(111)^{411}$ and is expected to take place on the polar $\mathrm{RhO}_{2} / \mathrm{Rh}(111)$ surface as well. ${ }^{233}$ Adsorption on polar oxide films might therefore be exploited to produce ordered arrangements of atoms, molecules and even metal aggregates, being a crucial precondition for different applications in microelectronics, chemical sensing and catalysis. Possible consequences of the distinct adsorption properties of polar oxide surfaces for their chemical reactivity need to be explored in future.

\subsubsection{Adsorption on structural peculiarities of thin oxide films}

Another important aspect in the binding behavior of oxide thin films is the occurrence of completely new adsorption sites that have no counterpart in the respective bulk materials. The most apparent representatives in this class are structural defects that result from the film growth on a metal support, and in particular from the relaxation of misfit-induced lattice strain. One example is the adsorption behavior controlled by Moiré and coincidence structures, as already introduced in the former section. Also the adsorption on misfitdislocations and domain boundaries is specific to thin oxide films, but will be discussed only in the following Chapter 4.4.4. The focus of this paragraph lies on structural peculiarities of thin oxide films that directly involve the metal support, e.g. well-defined openings in the film that give access to the metal-oxide interface. The associated adsorption phenomena are consequently restricted to ultrathin films with a thickness of a few layers only, and shall be summarized below.

The alumina film that can be prepared by oxidation of the $\mathrm{Ni}_{3} \mathrm{Al}(111)$ surface ${ }^{175}$ was the first example, where metal adsorption was found to be triggered by a regular hole-pattern in the oxide film. The template effect was observed after deposition of $\mathrm{Cu}, \mathrm{Pd}$ and $\mathrm{Au}-\mathrm{Pd}$ onto the oxide surface at room temperature. ${ }^{412,413}$ It resulted in a hexagonal cluster arrangement with $4.1 \mathrm{~nm}$ lattice constant, in good correspondence to the size of the oxide unit cell (Fig. 4.29). Surprisingly, for other ad-materials, e.g. vanadium, a three-times higher cluster density was 
observed on the same surface, although the long-ranged hexagonal order was maintained. Subsequent topographic and spectroscopic STM measurements on the bare alumina/ $\mathrm{Ni}_{3} \mathrm{Al}(111)$ film revealed the presence of two well-ordered defect structures with different periodicities, which seemed to be responsible for the self-assembly of the metal deposits. ${ }^{414,415}$

\section{Fig. 4.29:}
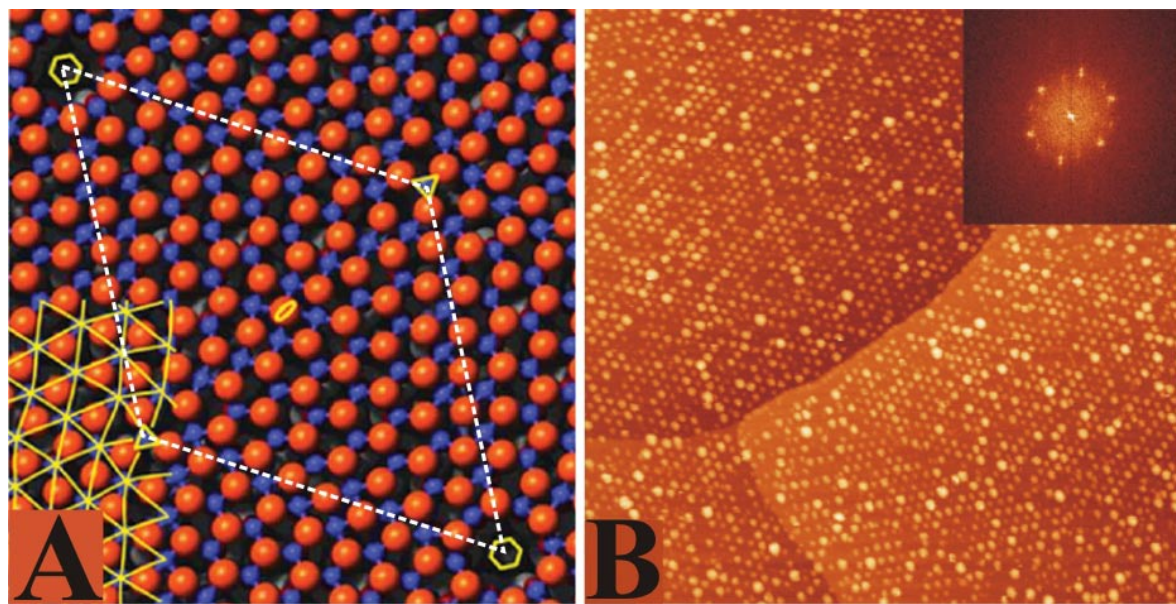

(A) Structure model of the alumina film on $\mathrm{Ni}_{3} \mathrm{Al}(111)$ (O ions: red, $\mathrm{Al}$ ions: blue). The corner holes and the oxygen defect are marked by the yellow hexagons and the ellipse, respectively. ${ }^{176}$ (B) STM image of an ordered array of $\mathrm{Au}-\mathrm{Pd}$ particles on alumina / $\mathrm{Ni}_{3} \mathrm{Al}(111)$ formed upon metal exposure at $300 \mathrm{~K}\left(200 \times 200 \mathrm{~nm}^{2}\right)$. The inset displays a Fourier transformation of the image. The presence of six superstructure spots emphasizes the perfect long-range order in the particle distribution. ${ }^{43}$

The nature of these defects and the true origin of the template effect was, however, only determined after setting up a structure model for the oxide film in a combined STM and DFT study (Fig. 4.29A). ${ }^{176}$ Similar to the alumina film on $\mathrm{NiAl}(110),{ }^{31}$ the $\mathrm{Ni}_{3} \mathrm{Al}$-supported oxide film is composed of four atomic planes. The surface is formed by a close-packed layer of oxygen ions, followed by a hexagonal $\mathrm{Al}^{3+}$ and a structurally identical $\mathrm{O}^{2-}$ layer underneath. The interfacial oxide layer comprises $\mathrm{Al}^{3+}$ hexagons, reflecting the lattice symmetry of the $\mathrm{Ni}_{3} \mathrm{Al}(111)$. Two distinct lattice irregularities were identified within the unit cell. (i) At two positions, three interfacial hexagons are replaced by three pentagons and a triangle, whereby a four-fold coordinated $\mathrm{O}$ ion is inserted into the lattice in deviation to the three-fold oxygen coordination in bulk alumina. (ii) At the corners of the unit cell, a complete $\mathrm{AlO}_{3}$ unit at the interface and the corresponding surface atoms are missing, producing a hole of approximately $6 \AA$ diameter in the film that reaches down to the metal surface. Both defects enable a slight increase of the average $\mathrm{O}-\mathrm{O}$ distance and thus contribute to the relaxation of lattice strain exerted by the metal substrate. The stoichiometry-defect associated with the over-coordinated oxygen ion offers an attractive binding site for metals with high oxygen affinity and is thus responsible for the self-organization of vanadium and manganese clusters. ${ }^{412}$ The regular 
holes in the oxide surface, on the other hand, can be penetrated by selected metal atoms, which strongly bind to the alumina- $\mathrm{Ni}_{3} \mathrm{Al}(111)$ interface and serve as anchoring sites for larger metal clusters. By this means, an ordered pattern of ad-clusters forms, whose number density corresponds to the density of holes in the alumina film. The fact that not every atom type is able to fill the corner holes is attributed to an element-specific diffusion barrier across the hole-boundary that can only be overcome by chemically inactive elements such as $\mathrm{Cu}$ and Pd. ${ }^{176}$
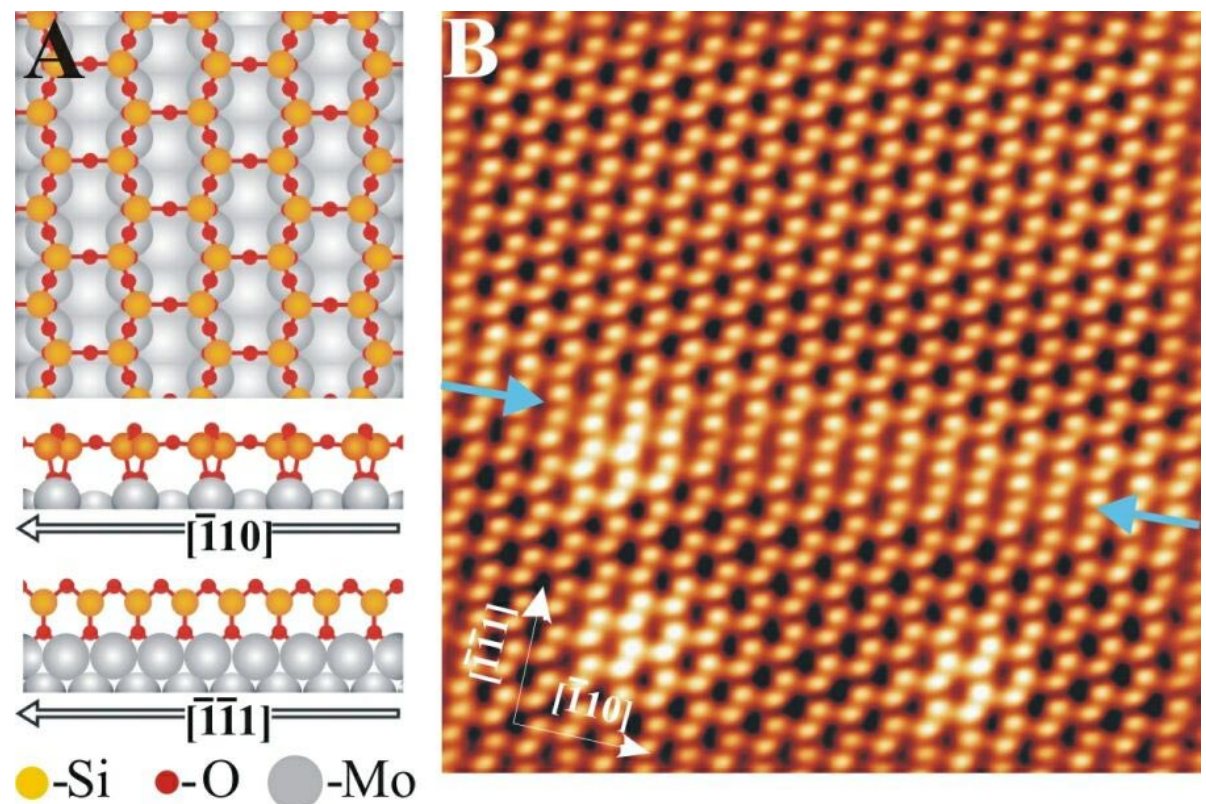

Fig. 4.30:

(A) Structure model of the silica thin film on Mo(112), top-view and side-views. ${ }^{422}$ (B) STM topographic image of the oxide film $\left(1.0 \mathrm{~V}, 10 \times 10 \mathrm{~nm}^{2}\right)$. The two arrows mark a domain boundary that runs from the left to the right.

A conceptually similar adsorption mechanism that does however not lead to self-organization phenomena is found on ultrathin titania and silica films grown on $\operatorname{Pt}(111)^{416}$ and $\operatorname{Mo}(112)$, respectively. ${ }^{417,418,419}$ Also here, strong binding sites are available at the metal-oxide interface, whereas the oxide surface does only weakly interact with atomic or molecular adsorbates due to a saturated bond structure. In the case of silica, the interfacial binding sites are accessible due to the porous nature of the film that closely resembles the one of zeolites and silicates. ${ }^{420}$ The oxide film consists of a single layer of $\mathrm{Si}_{-} \mathrm{O}_{4}$ tetrahedrons, whereby three of the oxygen atoms are bridge-bonded to neighboring $\mathrm{Si}$ atoms in the plane and the fourth one sits below the Si and anchors the film to the Mo support (Fig. 4.30). ${ }^{421,422}$ The -Si-O- surface units are arranged in hexagonal rings, which enclose a hole of 3-4 $\AA$ diameter and open towards a nano-pore at the metal-oxide interface. The resulting honeycomb structure of the oxide layer 
is clearly resolved in STM topographic images as the one shown in Fig. 4.30. The silica surface is chemically inert, as the $\mathrm{Si}$ ions are formally in the 4+ oxidation state and $\mathrm{Si}$ dangling bonds are absent. The main structural defects are [110]-oriented domain boundaries that form between two oxide patches shifted by a [111]-oriented Mo unit vector (see arrows in Fig. 4.30). To bridge this displacement vector, the regular six-membered rings are replaced by eight-membered entities at the boundary, being characterized by a two times larger central opening. $^{422}$
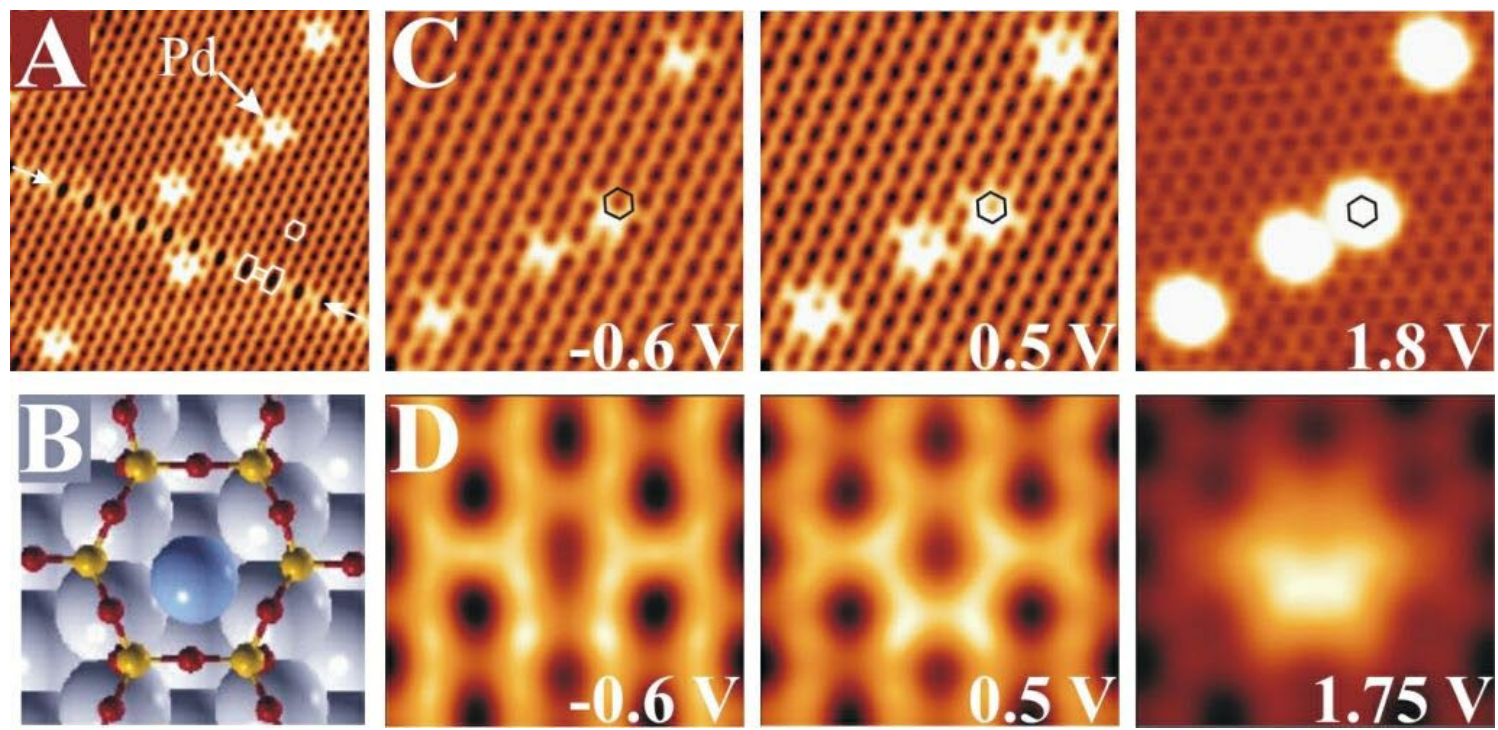

Fig. 4.31:

(A) STM image $\left(0.5 \mathrm{~V}, 13 \times 13 \mathrm{~nm}^{2}\right)$ of single Pd atoms on $\mathrm{SiO}_{2}$ on $\mathrm{Mo}(112)$. (B) Structure model of the Pd adsorption site (O: small, red; Si: small, yellow and Mo: large, blue spheres). Bias dependence of the topographic contrast of Pd atoms in (C) experimental $\left(5 \times 5 \mathrm{~nm}^{2}\right)$ and (D) simulated STM images $\left(1.8 \times 1.6 \mathrm{~nm}^{2}\right) .{ }^{418}$

Deposition of single Pd atoms onto the silica/Mo(112) film at $20 \mathrm{~K}$ results in STM images that seem to be incompatible with a binding of metal atoms to the oxide surface, as presented in the Chapters $\underline{4.4 .1}$ and $\underline{4.4 .2 .} .^{374,375,391}$ Instead of circular protrusions, distinct hexagonal stars appear in the STM images taken at $+0.5 \mathrm{~V}$ sample bias after Pd adsorption (Fig. 4.31A). ${ }^{418}$ Their resemblance to the -Si-O- hexagons in the pristine oxide lattice suggests that not the adatom itself, but a Pd-induced modification of the silica electronic structure is responsible for this particular contrast. The Pd-related adsorption features undergo distinct contrast changes as a function of sample bias (Fig. 4.31C). Whereas faint elongated maxima are visible at negative bias, the features transform into hexagonal stars with increasing positive bias and are imaged as large Gaussian protrusions of $0.7 \AA$ height above $+1.75 \mathrm{~V}$. 
A similar adsorption behavior is revealed upon silver deposition onto the silica surface at cryogenic temperatures (not shown). ${ }^{423}$ Also there, distinct hexagonal stars emerge in the topographic images at low positive bias, which develop into circular maxima above $+1.25 \mathrm{~V}$. Both Pd- and Ag-related features are randomly distributed on the silica surface and show no (or little for Ag) preference for a binding to the domain boundaries in the oxide film.
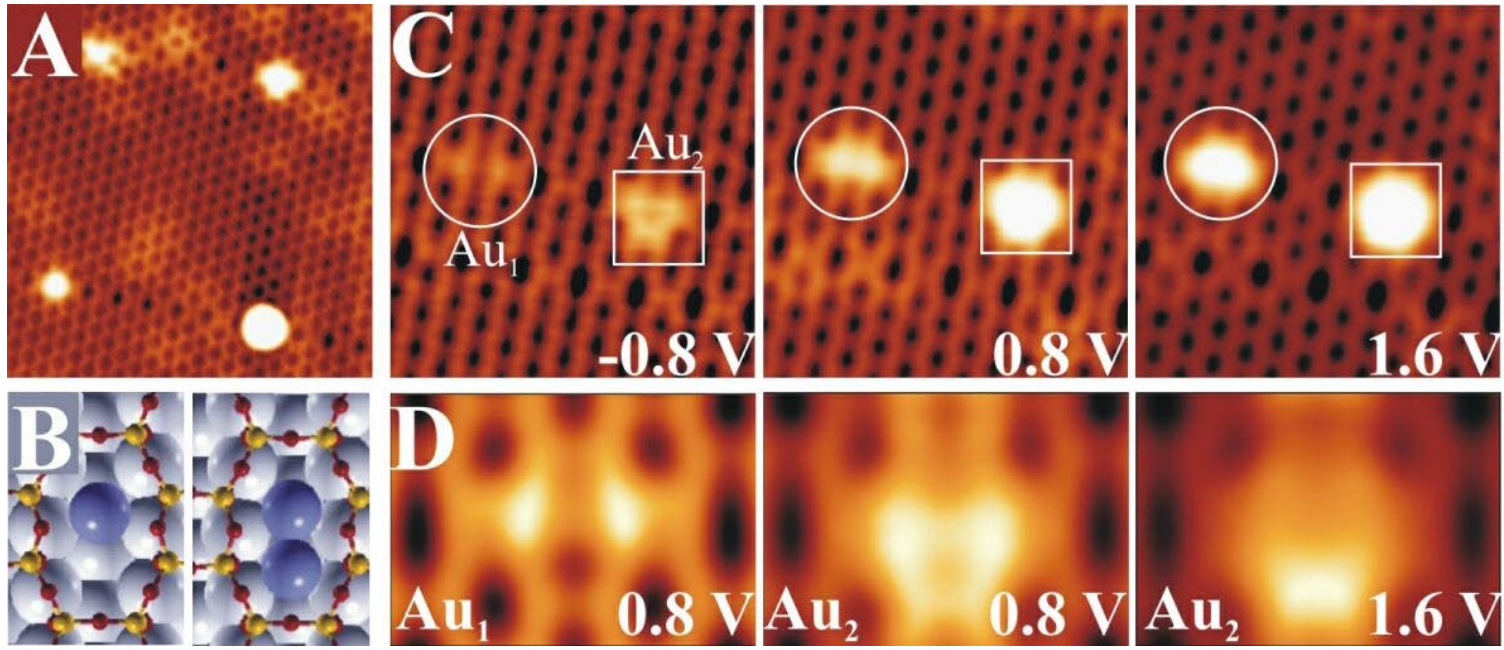

Fig. 4.32: (A) STM image $\left(0.5 \mathrm{~V}, 13 \times 13 \mathrm{~nm}^{2}\right)$ of gold on silica/Mo(112). (B) Structure model of an $\mathrm{Au}$ monomer and dimer in an octagonal -Si-O- ring located along the silica domain boundaries $(\mathrm{O}$ : small, red; Si: small, yellow and Mo: large, blue spheres). Bias dependence of the topographic contrast for different $\mathrm{Au}$ aggregates in (C) experimental $\left(4.5 \times 4.5 \mathrm{~nm}^{2}\right)$ and (D) simulated STM images $\left(2.1 \times 1.4 \mathrm{~nm}^{2}\right)$.

A completely different behavior is revealed for Au adsorption on the oxide film (Fig. 4.32). Whereas no surface modifications are visible on defect-free silica patches, differently-sized protrusions develop along the domain boundaries upon Au exposure. ${ }^{418}$ The smallest Auinduced feature affects only a single eight-membered ring in the line defect and is tentatively assigned to a single adatom. It shows a similar bias-dependent contrast as the Pd atom (Fig. 4.32C). At small negative bias, only the outer part of the -Si-O- octagon appears bright, leaving the middle of the pore as a depression. With increasing positive bias, the contrast gradually moves towards the center and finally evolves into a bright elliptical protrusion localized on top of the eight-membered ring. Also a second, asymmetric structure can be identified, which is attributed to an Au dimer interacting with the octagonal -Si-O- rings (Fig. 4.32C). Also in this case, the ring center is dark at negative bias, but turns into a bright protrusion in images taken above $+0.8 \mathrm{~V}$ sample bias. With increasing gold exposure, larger aggregates with spherical shapes start to decorate the domain boundaries, while the silica terraces in between remain devoid of gold-related species. 
The unusual adsorption characteristic of $\mathrm{Pd}, \mathrm{Ag}$ and $\mathrm{Au}$ atoms on the silica film can be elucidated with DFT calculations. ${ }^{381}$ Pd atoms are able to penetrate the openings in the sixmembered rings with virtually no barrier and bind to a short Mo-Mo bridge site at the interface (Fig. 4.31B). The resulting adsorption energy is with $3.3 \mathrm{eV}$ almost ten times larger than on the silica surface, explaining the absence of surface-bound species. The inserted Pd atom interacts not only with the Mo electronic states, but modifies the electronic structure of the hosting silica ring in addition. The unoccupied $P d 5 s$ orbital hereby hybridizes with the $2 p$ states of the surface oxygen atoms, which leads to a local increase of the unoccupied state density at the oxygen sites (Fig. 4.33). The availability of these new electronic states above the Fermi level gives rise to the distinct star-like contrast in empty-state STM images, as the six oxygen atoms of the hosting -Si-O- ring appear brighter than in regular hexagons (Fig. 4.31). ${ }^{418}$ The contrast change towards a single protrusion at $+1.75 \mathrm{~V}$ occurs when the $P d 5 \mathrm{~s}$ orbital itself becomes available for electron transport, making the Pd atom inside the nanopore visible in the STM. According to the DFT results, the $P d 5$ s resonance peaks at $+2.0 \mathrm{eV}$, which is compatible with the bias range at which the star-like contrast disappears in favor of the circular protrusion. The energy position of the $P d 5 s$ orbital has been verified also with STM conductance spectroscopy, which gives a faint $\mathrm{dI} / \mathrm{dV}$ shoulder slightly below the silica conduction band at $2.3 \mathrm{~V}$ for the inserted Pd atom (Fig. 4.33). The binding behavior of silver is comparable, because also the Ag atoms are able to penetrate the silica top-layer and attach to the metal-oxide interface with $1.6 \mathrm{eV} .^{423}$ The penetration barrier is with $0.3 \mathrm{eV}$ larger than for Pd, for which a value below $0.1 \mathrm{eV}$ has been computed. The star-like contrast of the inserted Ag atoms at low bias is caused again by hybridization between the $\mathrm{Ag} 5 \mathrm{~s}$ and the $\mathrm{O}$ $2 p$ orbitals. It changes into a bright circular protrusion when the $\mathrm{Ag} 5 \mathrm{~s}$ resonance state, being calculated at $+1.6 \mathrm{eV}$, becomes directly available for electron transport. In close correspondence with the computed LDOS, a pronounced peak at $2.05 \mathrm{~V}$ is observed in the dI/dV spectra taken above an interfacial Ag atom (Fig. 4.33). 

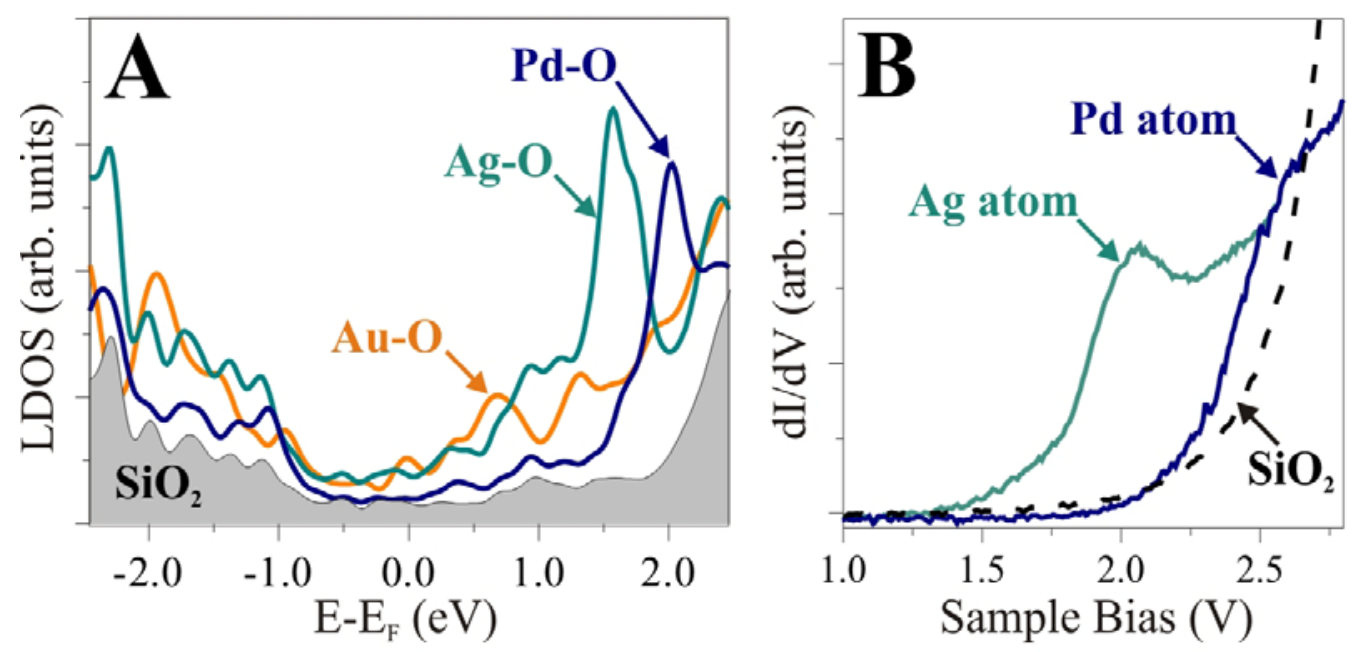

Fig. 4.33

(A) Total LDOS of the pristine silica film (grey) and plots of the hybrid states formed between the sorbitals of inserted Pd, Ag and Au atoms and the $O 2 p$ states of the surface oxygen species. (B) dI/dV spectra of the pristine oxide film and inserted Pd and Ag atoms, showing similar resonances as in (A).

In sharp contrast to $\mathrm{Pd}$ and $\mathrm{Ag}$, the penetration barrier for $\mathrm{Au}$ atoms is with $0.9 \mathrm{eV}$ too high to be overcome upon atom deposition at $20 \mathrm{~K}$. Even activated penetration at higher temperature is not feasible, because $\mathrm{Au}$ desorption from the silica surface requires only $0.1 \mathrm{eV}$ and consequently occurs before penetration. As a result, Au atoms cannot be incorporated into the defect-free silica patches, although interfacial binding would be highly preferred from the energetic point of view $\left(E_{B}=2.04 \mathrm{eV}\right) .{ }^{381}$ The physisorbed $\mathrm{Au}$ atoms on the oxide surface hence perform rapid diffusion even at the low temperature of the experiment, until they become trapped at the domain boundaries in the oxide film. There, a penetration of the eightmembered defect-rings is possible without substantial energy barrier and the Au reaches highenergy binding sites at the metal-oxide interface below the -Si-O- octagons. In this adsorption configuration, the $A u$ 6s orbital strongly hybridizes with the Mo states, while the interaction with the oxide LDOS is marginal due to the large energy mismatch. This explains why no Auinduced states are detectable in $\mathrm{dI} / \mathrm{dV}$ spectroscopy of the Au-silica system (Fig. 4.33). According to the DFT calculations, ${ }^{418}$ two Au atoms are able to occupy the nano-pore below a -Si-O- octagon (Fig. 4.32), while additional atoms can be attached above the pore. Domain boundaries in the silica film therefore serve as nucleation centers for Au nano-particles, in accordance to the experimental results. ${ }^{424}$

The difference in the penetration barrier of $\mathrm{Pd}, \mathrm{Ag}$ and $\mathrm{Au}$ atoms is not due to a geometric effect, as all three atoms have rather similar van-der-Waals radii with the Ag atom being tallest. ${ }^{425}$ The barrier height is in fact determined by an electronic repulsion, which the $2 p$ states of the surface oxygen ions exert on the filled orbitals of the incoming adatom. ${ }^{423}$ Hereby the s-like valence states play the dominant role due to their diffuse and spatially extended 
nature. The Pd atom has no valence s-electron, as the $P d 5 s$ orbital lies well above the Fermi level before and after penetration. It can therefore pass the -Si-O- rings in the silica top layer with little activation. The Ag, on the other hand, has a half-filled $5 s$ orbital in the gas phase, but is able to temporarily release this electron into the support when passing the nano-hole in the silica film. The hence positively charged atom (Bader charge of $+0.6|\mathrm{e}|$ ) experiences only a small repulsion with the oxide charge density and the penetration barrier remains small. In contrast, the highly electronegative Au stays neutral when approaching the silica surface. Its half-filled $\mathrm{Au} 6 \mathrm{~s}$ state therefore experiences a considerable repulsive interaction with the filled $O 2 p$ states, which inhibits the atom to pass the opening in the regular -Si-O- rings. The selectivity of the silica film in enabling specific atoms to penetrate the oxide surface is therefore exclusively governed by the electronic properties of the adsorbates, in particular by the spatial extension and electron filling of their valence states. Following this conclusion, low penetration barriers are predicted for small and electro-positive alkali metals, e.g. for Li and $\mathrm{Na}$, whereas the highly electronegative Pt will be unable to pass the oxide surface-holes in analogy to the Au species. ${ }^{378}$
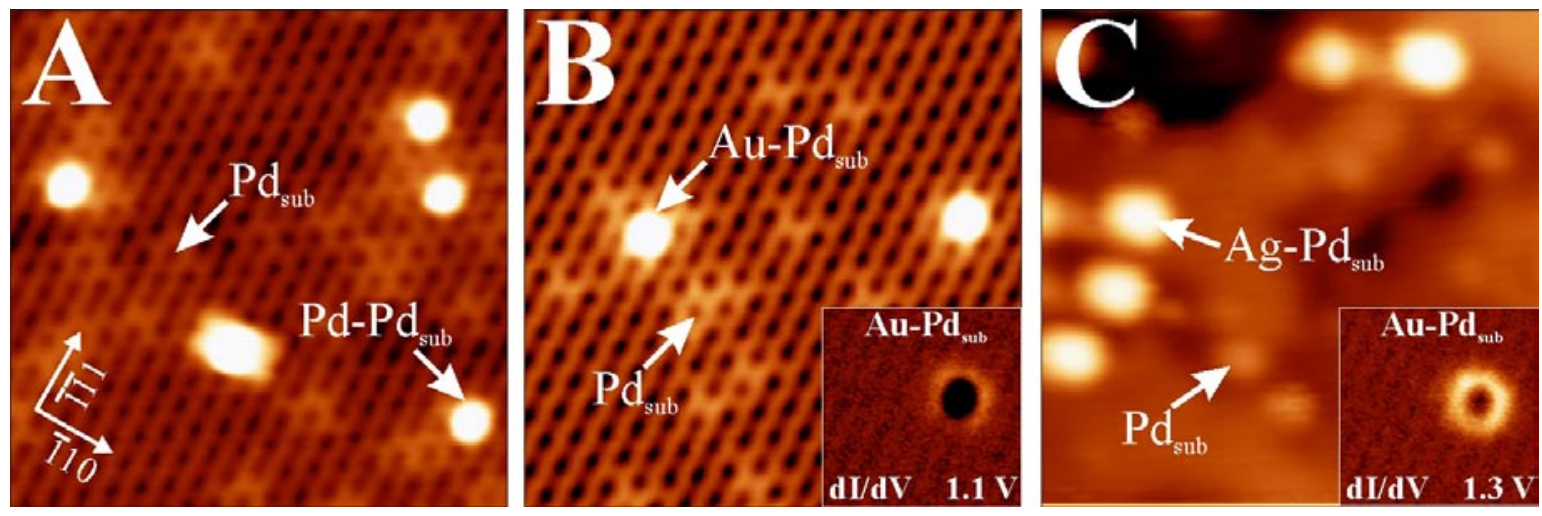

Fig. 4.34:

STM topographic image of $\mathrm{SiO}_{2} / \mathrm{Mo}(112)$ taken after insertion of $\mathrm{Pd}_{\text {sub }}$ species and subsequent deposition of (A) single Pd (0.5 V) (B) Au (0.5 V) and (C) Ag atoms (1.9 V, $\left.14 \times 14 \mathrm{~nm}^{2}\right)$. The bright ad-species in all three images are assigned to metal adatoms anchored to the inserted $\mathrm{Pd}_{\text {sub }}$. The insets in (B) and (C) depict $\mathrm{dI} / \mathrm{dV}$ maps of an $\mathrm{Au}-\mathrm{Pd}_{\text {sub }}$ complex at two different bias voltages. The ring-like conductance enhancement results from a perturbation of the silica electronic structure due to a lattice distortion induced by the $\mathrm{Au}^{426}$

The possibility to insert single metal atoms into silica/Mo(112) films, as demonstrated above, opens the unique possibility to modify the adsorption characteristic of the originally inert oxide film. As the inserted atoms remain close to the silica surface, they might be able to anchor adsorbates that would not bind to the film otherwise. As the nature and density of 
those sub-surface anchoring sites can be easily manipulated, the chemically modified silica film might represent a simple realization for a functionalized adsorption system.

The controlled anchoring of metal atoms has first been observed on a silica film that was altered by inserting $1 \times 10^{13}$ interfacial Pd atoms per $\mathrm{cm}^{-2}$ (referred to as $\mathrm{Pd}_{\text {sub }}$ in the following). ${ }^{426}$ Subsequent deposition of Pd, Ag and Au monomers at $20 \mathrm{~K}$ onto the modified film was found to result in the formation of new ad-species on the oxide surface (Fig. 4.34). Neither their bias-independent spherical shapes, nor an apparent height of more than $2.0 \AA$ seemed to be compatible with the signature of inserted atoms that has been described before. Also the spectroscopic investigation of the newly formed ad-species revealed distinct differences with respect to the $\mathrm{dI} / \mathrm{dV}$ fingerprints of the sub-surface atoms. ${ }^{426}$ The adsorbate complexes were therefore assigned to $\mathrm{Pd}$, $\mathrm{Ag}$ or $\mathrm{Au}$ adatoms attached to a $\mathrm{Pd}_{\text {sub }}$ anchor inside the silica film. It should be emphasized at this point that none of these metal atoms are able to bind to the pristine film, as they either spontaneously penetrate the silica pores or diffuse to adjacent defect lines. ${ }^{418}$

The attachment of surface atoms to the $\mathrm{Pd}_{\text {sub }}$ anchors was verified with STM manipulation experiments, where selected adatoms had been removed from their surface binding sites via a moderate voltage pulse of 3-5 V. After desorption, the characteristic star-like signature of an inserted $\mathrm{Pd}_{\text {sub }}$ became visible in every case, suggesting that the $\mathrm{Pd}_{\text {sub }}$ anchor is indeed required to stabilize the adsorption complex. An image series demonstrating the detachment of a Pd adatom from a subsurface $\mathrm{Pd}_{\text {sub }}$ is presented in Fig. 4.35.
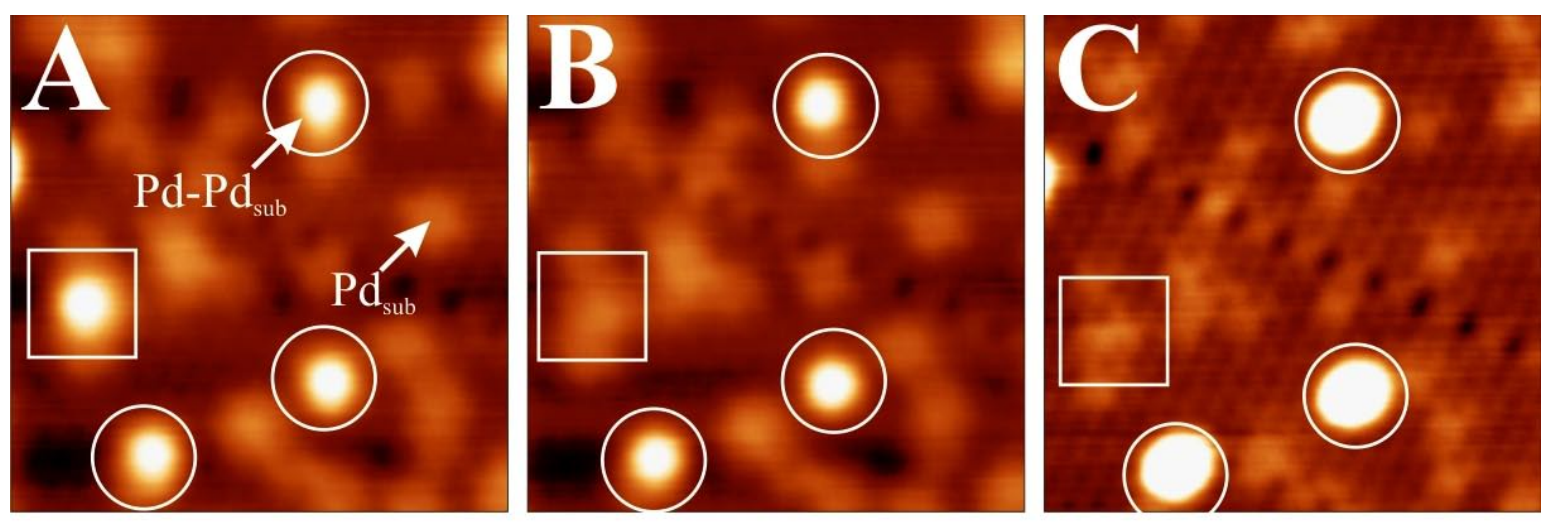

Fig. 4.35:

(A) STM image of $\mathrm{Pd}_{\text {sub }}$ and Pd-Pd $\mathrm{d}_{\text {sub }}$ species on $\mathrm{SiO}_{2} / \mathrm{Mo}(112)\left(1.0 \mathrm{~V}, 10 \times 10 \mathrm{~nm}^{2}\right)$. (B) As in (A) but after controlled removal of the adatom marked by the square. (C) As in (B) but with enhanced contrast to show the silica atomic structure. Below the removed atom, the $\mathrm{Pd}_{\text {sub }}$ that has initially anchored the adatom becomes visible. The circles indicate other $\mathrm{Pd}-\mathrm{Pd}_{\text {sub }}$ complexes as guides for the eye. 
Accompanying DFT calculations revealed that the anchoring strength of the inserted $\mathrm{Pd}_{\text {sub }}$ strongly depends on the chemical nature of the adsorbate. ${ }^{426}$ Although the $\mathrm{Pd}_{\text {sub }}$ forms a covalent bond with all the three ad-atoms, the binding capacity decreases from $1.14 \mathrm{eV}$ for a Pd surface-atom to 0.34 and $0.19 \mathrm{eV}$ for $\mathrm{Au}$ and $\mathrm{Ag}$ ad-species, respectively. The different interaction energies have a similar electronic origin as the penetration barrier of the respective metal atoms discussed before. The total binding strength to the silica surface results from a

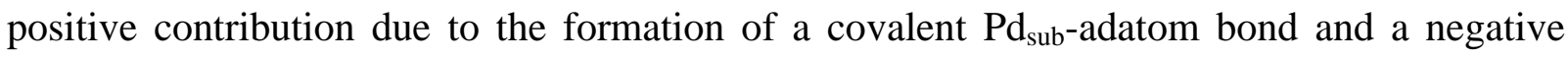
term due to the electronic repulsion that is exerted by the oxide charge density onto the filled adsorbate levels. The repulsive contribution hereby increases with the number of s-like valance electrons in the adatom. Consequently, the Pd atom with its empty Pd 5s orbital binds strongest to the sub-surface anchor, while the interaction is substantially weakened for an $\mathrm{Ag}$ atom with its singly occupied valence s-shell. The effect of electron-electron repulsion is, however, largest for Au, which accumulates an excess electron from the Mo support and becomes negatively charged on the silica surface. The mechanism of the charge transfer is identical to the one described in Chapter $\underline{4.4 .1}$ and includes dropping of the $A u$ 6s orbital below the silica/Mo(112) Fermi level and tunneling of an electron into the originally halffilled 6s state. ${ }^{186}$ The fact that the Au binding energy to a $\mathrm{Pd}_{\text {sub }}$ anchor is still comparable to the one of Ag reflects the stabilizing influence of the Au $d$-states that strongly hybridize with the $d$-levels of the $\mathrm{Pd}_{\text {sub. The }} \mathrm{Ag} d$-states, on the other hand, are far below the Fermi level and unable to participate in the $\mathrm{Pd}_{\text {sub }}$-Ag bond formation, explaining the small interaction strength in this case.

The attachment of a surface atom to a $\mathrm{Pd}_{\text {sub }}$ anchor is accompanied by a strong relaxation of the hosting -Si-O- ring to reduce the influence of steric repulsion with the $O 2 p$ states. In the case of Au with its doubly occupied 6s-orbital, the enlargement of the hole diameter amounts to roughly $5 \%$ and initiates an increase of the $\mathrm{Au}-\mathrm{Pd}_{\text {sub }}$ bond strength by more the $100 \%$ with respect to the non-relaxed geometry $(0.34 \mathrm{eV}$ versus $0.14 \mathrm{eV})$. The silica lattice distortion gives rise to a local perturbation of the oxide electronic structure that becomes manifest by a ring-like conductance enhancement around the Au adatom in $\mathrm{dI} / \mathrm{dV}$ maps taken between 1.0 and $1.3 \mathrm{~V}$ (see insets in Fig. 4.34).

The anchoring mechanism of different adatoms to inserted $\mathrm{Pd}_{\text {sub }}$ is therefore chemically selective as well. The largest interaction strength is hereby expected for adsorbates with a spatially confined charge density. The approach presented here can in principle be used to fabricate more complex aggregates, whereby the different atomic species have to be attached 
sequentially to the sub-surface anchors. Alternatively, the technique can be exploited to control the charge state of adsorbates in a desired way. For this purpose, surface anchors with electron acceptor or donor-like properties need to be inserted. The incorporation of $\mathrm{Li}, \mathrm{Na}$ and $\mathrm{K}$ atoms, for example, induces a considerable positive surface dipole that reduces the oxide work function and triggers a negative charging of adsorbates on the silica surface. ${ }^{378}$ Following this idea, the stabilization of anionic Au clusters has been demonstrated on a Lidoped silica film. The Au was found to bind even in the absence of surface defects in this case, reflecting the electrostatic and polaronic binding schemes that are available for charged ad-species. $^{379}$

The insertion of artificial binding sites into porous oxide films shares similarities to the wellknown doping approach of oxide materials via substitutional atoms. Prominent examples for the latter are the incorporation of $\mathrm{Li}$ and $\mathrm{Ti}$ into bulk $\mathrm{MgO}$ and $\mathrm{SiO}_{2}$, aiming for a modification of the oxide properties. ${ }^{381,427,428}$ However, while doping affects the global electronic and chemical parameters of the oxide material, embedding of suitable single-atom anchors modifies the binding behavior only locally. The surface properties of oxide materials can thus be adjusted in a more targeted way.

\subsubsection{Adsorption on defects in oxide thin films}

The second group of structural inhomogeneities that stimulate distinct adsorption schemes on thin oxide films comprises intrinsic defects in the oxide lattice. Relevant thin-film defects are point defects, in particular oxygen vacancies, and line defects, e.g. dislocation lines, domain boundaries and step edges. The concentration of point defects is usually small in thin oxide films thanks to a preparation in an excess of oxygen and at sufficiently high temperatures. ${ }^{48,143}$ This defect type needs therefore to be introduced artificially, e.g. via electron bombardment ${ }^{29,248,266}$ or mild ion sputtering. ${ }^{252}$ The formation of line defects, on the other hand, is directly connected with the growth of oxide materials on supports with a deviating lattice parameter, where they promote the release of misfit-induced strain in the oxide lattice (see Chapter 3.4). ${ }^{51,142,152,178}$ Adsorption on such growth-induced line defects has therefore no equivalence for the respective bulk materials.

The number of STM-based adsorption studies on single, well-defined point defects is surprisingly small. In most cases, the role of point defects in adsorption events was indirectly derived from the growth of metal deposits on flat oxide terraces in large distance to step edges and other apparent line defects. ${ }^{48,251,429}$ The assumption of a defect-mediated nucleation 
mechanism was thereby rationalized by the higher binding potential of oxygen vacancies compared to regular surface sites, as calculated for many bulk oxides with DFT. ${ }^{312,316,318}$ The actual atomic nature of the underlying defect and the dominant interaction mechanism remained therefore unclear in most of these studies. A better insight into defect-mediated adsorption on thin oxide films was gained, when surface defects had been introduced in a controlled manner, for instance by reducing the film at elevated temperature or exposing it to energetic electrons to stimulate oxygen desorption. ${ }^{430}$ In this kind of experiments, nucleation on oxide terraces could directly be related to the availability of oxygen vacancies. A typical example is the interaction of $\mathrm{Au}$ with defect-poor versus defect-rich surfaces of a $\mathrm{CeO}_{2} / \mathrm{Ru}(0001)$ film (Fig. 4.36). ${ }^{431}$ Whereas the Au exclusively nucleates along step edges in the stoichiometric film, $20 \%$ of the particles develop on the terraces after creation of oxygen vacancies via mild oxide reduction. In selected cases, the associated lattice defects had even been identified in the STM prior to metal deposition (Fig. 4.36). ${ }^{261}$
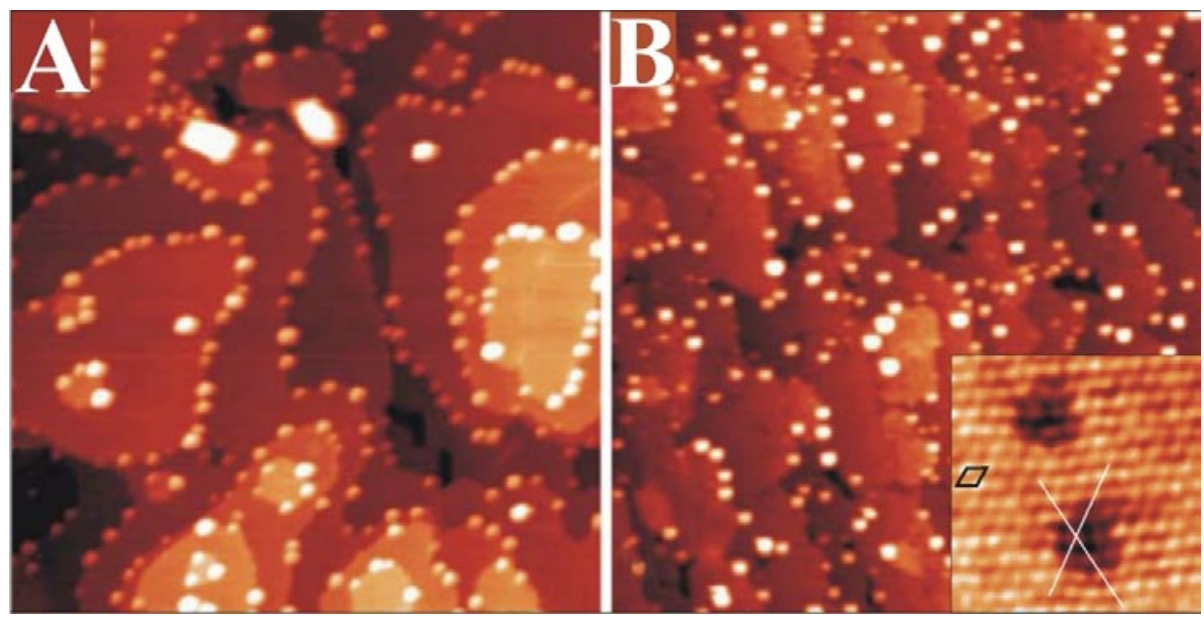

\section{Fig. 4.36:}

STM images of $0.1 \mathrm{ML}$ Au deposited on (A) the fully oxidized and (B) the partially reduced $\mathrm{CeO}_{2}$ film grown on $\mathrm{Ru}(0001)$ $\left(4.1 \mathrm{~V}, 150 \times 150 \mathrm{~nm}^{2}\right)$. The inset in (B) shows two defects in the $\mathrm{CeO}_{2}$ surface, being assigned to oxygen vacancies. ${ }^{261 \text {, }}$ 431

An unambiguous correlation between atomically-defined point defects and a distinct binding behavior of atomic or molecular species has been provided for vanadia thin-films grown on $\mathrm{Au}(111) .{ }^{262,432,433}$ The $\mathrm{V}_{2} \mathrm{O}_{3}(0001)$ surface exposes a closed-packed layer of vanadyl groups when prepared at intermediated $\mathrm{O}_{2}$ chemical potentials, ${ }^{26}$ but forms a bulk-truncated $\mathrm{O}$ or $\mathrm{V}$ termination at strongly oxidizing or reducing conditions, respectively. ${ }^{224,225}$ The vanadyltermination is chemically inert due to the closed-shell electronic structure of the $\mathrm{V}=\mathrm{O}$ groups, which renders surface defects promising candidates for adsorption events. ${ }^{434}$ Two kinds of point defects have been identified in the surface, namely vanadyl defects where a complete $\mathrm{V}=\mathrm{O}$ group is missing and oxygen defects with only the vanadyl oxygen being detached from the $\mathrm{V}=\mathrm{O}$. Whereas the former defect type occurs naturally in the as-prepared film, the latter one needs to be introduced via electron bombardment. ${ }^{29,224}$ Particularly the oxygen defects 
have been connected with the catalytic activity of the vanadia surface, for instance in the oxidation of methanol. ${ }^{432}$
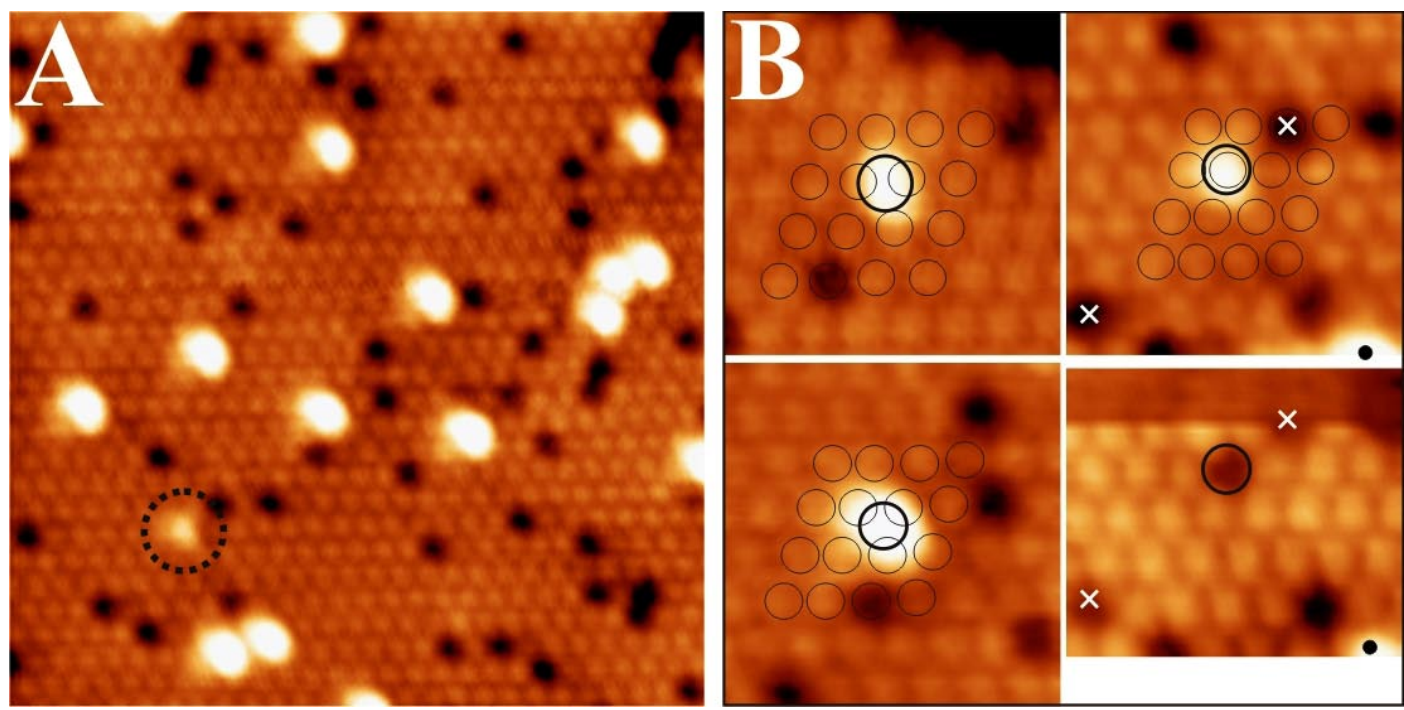

Fig. 4.37:

(A) STM image of the vanadyl-terminated $\mathrm{V}_{2} \mathrm{O}_{3}$ surface covered with $0.01 \mathrm{ML}$ of $\mathrm{Au}(-0.9 \mathrm{~V}, 13 \times 13$ $\mathrm{nm}^{2}$ ). The dark holes are missing $\mathrm{V}=\mathrm{O}$ groups in the surface. The Au atom marked by the circle is localized in such a vacancy site. (B) Close-up STM images of Au atoms in bridge and hollow positions (left) and in a $\mathrm{V}=\mathrm{O}$ vacancy site (right). The latter position is verified by removing the atom in a lowbias scan, uncovering the surface defect underneath. The dot/crosses are guides to the eye to identify similar sites in the two images on the right panel $\left(-0.5 \mathrm{~V}, 3.5 \times 3.5 \mathrm{~nm}^{2}\right){ }^{262}$

The role of point defects for the adsorption behavior of vanadyl-terminated $\mathrm{V}_{2} \mathrm{O}_{3}(0001)$ has been explored by depositing small amounts of Au at $10 \mathrm{~K} \cdot{ }^{262}$ STM topographic images of the surface after preparation display the hexagonal array of the $\mathrm{V}=\mathrm{O}$ groups as well as dark spots that cover $5-7 \%$ of the regular $\mathrm{V}=\mathrm{O}$ lattice sites and are assigned to point defects (Fig. 4.37A). ${ }^{223,224,225}$ In addition, single Au atoms appear as bright protrusions of 0.9-1.1 $\AA$ height, coexisting with a few Au aggregates of larger apparent size. Occasionally, a third surface species is detected, which shows a strongly bias-dependent contrast and is imaged as rather faint protrusion at $-0.9 \mathrm{~V}$ imaging bias (see circle in Fig. 4.37A). Atomically resolved STM images enable an identification of the adsorption sites of the different Au species. Regular adatoms, referred to as $\mathrm{Au}_{\mathrm{br}}$, occupy high-coordinated hollow and bridge sites in the $\mathrm{V}=\mathrm{O}$ lattice. A clear distinction between both positions is sometimes difficult due to the diffuse appearance of Au-related protrusions in the STM (Fig. 4.37B). The faint surface species, on the other hand, are always in an on-top position of the $\mathrm{V}=\mathrm{O}$ lattice. Because Au attachment to a $\mathrm{V}=\mathrm{O}$ group seems unlikely, those features are associated with Au atoms bound to surface defects $\left(\mathrm{Au}_{\mathrm{d}}\right)$. Experimental evidence for this assumption is obtained by displacing such an adatom in a low-bias STM scan, whereby the characteristic signature of a surface defect 
becomes visible (Fig. 4.37B). In contrast to the established picture, ${ }^{37}$ the abundance of defectbound $\mathrm{Au}_{\mathrm{d}}$ atoms is surprisingly small and a large number of surface vacancies remains empty after Au exposure.

Regular and defect-bound Au atoms are also distinguished via their conductance spectra (Fig. 4.38A). The bare, vanadyl-terminated oxide film exhibits a region of zero-conductance around the Fermi level, which reflects the $\mathrm{V}_{2} \mathrm{O}_{3}$ correlation gap in the $V$-3d band that opens below the Mott-transition at $150 \mathrm{~K} \cdot{ }^{263,435,436}$ The gap size amounts to $0.5-0.7 \mathrm{eV}$, and exhibits small spatial modulations due to local differences in the stoichiometry of the individual oxide grains (see also Fig. 3.7). ${ }^{194}$ The surface defects, on the other hand, have little influence on the $\mathrm{dI} / \mathrm{dV}$ spectra, manifesting the bulk character of the vanadia band gap. ${ }^{26,194}$ Gold atoms on regular lattice sites $\left(\mathrm{Au}_{\mathrm{br}}\right)$ do not produce new features in the conductance curves and only the valence band onset of $\mathrm{V}_{2} \mathrm{O}_{3}$ appears shifted towards $\mathrm{E}_{\mathrm{F}}$ in the vicinity to certain adatoms (see for instance the $\mathrm{Au}_{b r}{ }^{*}$ species in Fig. 4.38A). The shifted onset is however not a peculiarity of the adsorbate but of the oxide film below, and manifests the binding preference of Au atoms to oxide regions where the valence band is closer to the Fermi level. The associated increase of the local state density in the oxide volume below the binding site enables long-ranged polarization and dispersive forces between adatom and support, which add to the short-ranged chemical interactions. In contrast to regularly bound species, $\mathrm{Au}_{\mathrm{d}}$ atoms on defect sites show a prominent $\mathrm{dI} / \mathrm{dV}$ peak between +0.2 and $+0.4 \mathrm{~V}$ that is located inside the oxide band gap (Fig. 4.38). The $d I / d V$ spectra of small Au clusters are finally characterized by a set of peaks at positive bias, indicating the development of quantized electronic states in the confined cluster volume (Fig. 4.38, top spectrum).

The experimental adsorption behavior of gold on the vanadyl-terminated $\mathrm{V}_{2} \mathrm{O}_{3}$ surface can be compared to the results of the DFT calculations. ${ }^{262}$ On the defect-free $\mathrm{V}=\mathrm{O}$ surface, Au atoms preferentially occupy bridge sites between two vanadyl groups, while Au in adjacent hollow positions spontaneously relaxes into the bridge configuration (Fig. 4. 38B). It is interesting to note that the resulting O-Au-O binding geometry resembles the typical building blocks of bulk $\mathrm{Au}_{2} \mathrm{O} .{ }^{437}$ The binding energy of the $\mathrm{V}=\mathrm{O}$ bridge-bonded $\mathrm{Au}$ amounts to $1.81 \mathrm{eV}$ and the interaction is dominated by the formation of an $\mathrm{Au} 5 \mathrm{~d}-\mathrm{O} 2 \mathrm{p}$ hybrid states at $-1.6 \mathrm{eV}$ and two mixed $\mathrm{Au} 6 \mathrm{~s}-\mathrm{O} 2 \mathrm{p}$ states at -1.6 and $+1.5 \mathrm{eV}$, respectively (Fig. 4.38B). This finding perfectly reproduces the experimental situation, where regular-bound $\mathrm{Au}_{\mathrm{br}}$ atoms are found to occupy high-coordinated $\mathrm{V}=\mathrm{O}$ lattice sites. Also the absence of $\mathrm{dI} / \mathrm{dV}$ peaks in the experimental 
conductance spectra is supported by the calculated LDOS, which does not predict the occurrence of Au-induced states inside the oxide band gap.
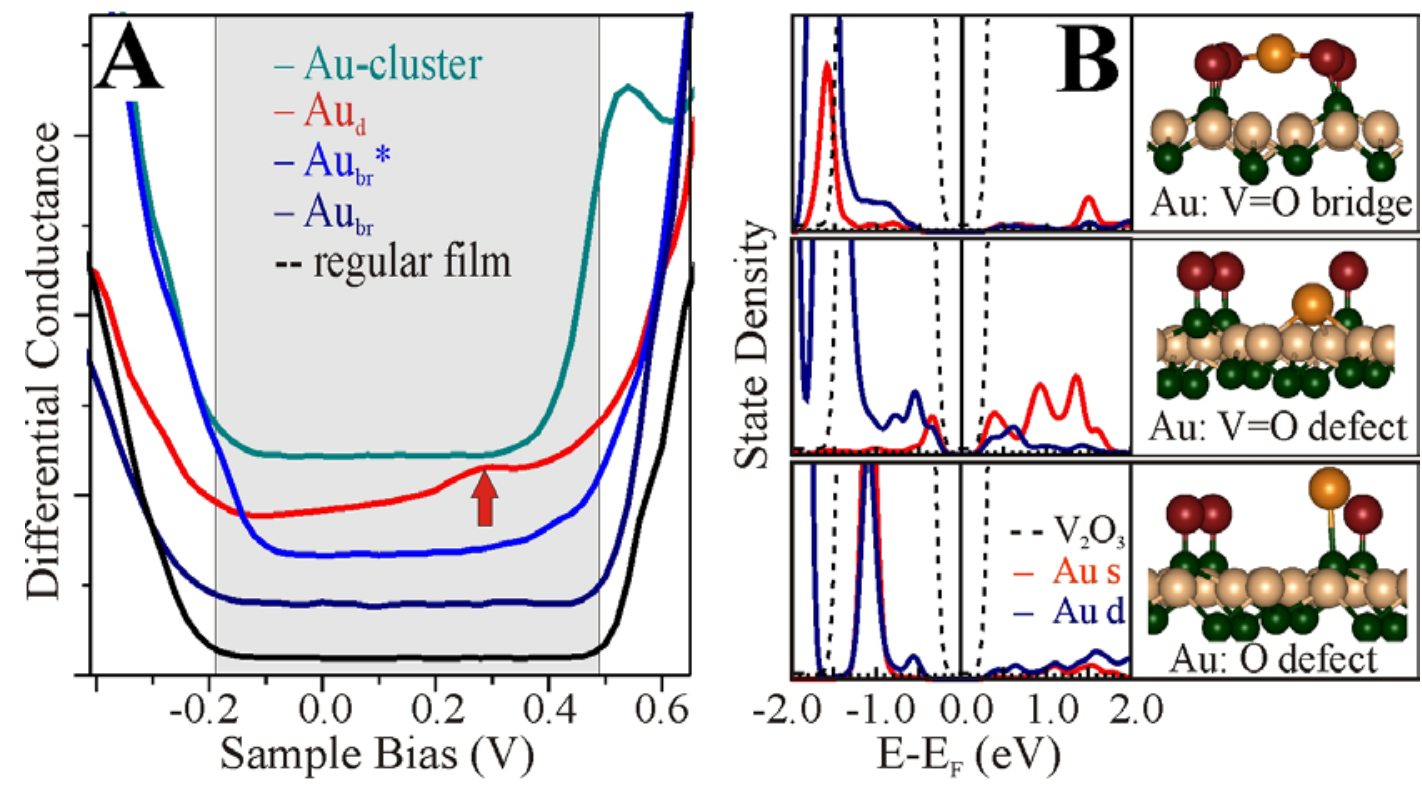

Fig. 4.38:

(A) $\mathrm{dI} / \mathrm{dV}$ spectra of $\mathrm{V}=\mathrm{O}$ terminated $\mathrm{V}_{2} \mathrm{O}_{3}$, regular and defect-bound $\mathrm{Au}$ atoms and a small cluster. The gap region is marked in grey. (B) Calculated LDOS and structure models for Au atoms in three different binding configurations on the $\mathrm{V}=\mathrm{O}$ terminated $\mathrm{V}_{2} \mathrm{O}_{3} \cdot{ }^{262}$

On the defective surface, two additional adsorption sites become available for the Au atoms. On the vanadyl defect with the complete $\mathrm{V}=\mathrm{O}$ group being removed, the adatom binds with $1.03 \mathrm{eV}$ in a three-fold hollow sites of the thus exposed bulk-like O layer (Fig. 4.38B). As in the $\mathrm{V}=\mathrm{O}$ bridge configuration, hybridization between the $A u 5 d$ and $O 2 p$ states stabilizes the adatom, which becomes positively charged upon adsorption (+0.63|e|). The $A u$ ss orbital only weakly participates in this interaction and its filled and unfilled components remain close to the Fermi level. A similar situation is encountered for Au attachment to other inert oxide surfaces. ${ }^{316,380}$ In contrast, if only the vanadyl oxygen is removed, the Au binds with $2.04 \mathrm{eV}$ to the hence unsaturated $\mathrm{V}$ ion. The strong bonding in this case is mediated by $V 3 d-\mathrm{A} u$ 6s / $5 d$ hybridization, which shifts the center of the $A u 6 s / 5 d$ level to $-1.2 \mathrm{eV}$ below $\mathrm{E}_{\mathrm{F}}$ (Fig. 4.38B).

Based on the two defect-mediated binding scenarios, the experimentally observed Au-defect interaction can be elucidated. The small abundance of defect-bound Au atoms is only compatible with an adsorption to $\mathrm{V}=\mathrm{O}$ vacancies, where the binding energy is lower than on regular surface sites. The large binding potential of the chemically-active $\mathrm{O}$ defects would otherwise lead to an immediate occupation of all defects with Au atoms, in clear contrast to 
the experimental observations. Apparently, O defects are not present in the as-prepared oxide film, which is in agreement with earlier statements made in the literature. ${ }^{29,224}$ The assignment of the surface defects to missing $\mathrm{V}=\mathrm{O}$ groups is also corroborated by the spectroscopic data, which display an Au defect-state around $+0.3 \mathrm{eV}$. Such Au-induced gap state is only present in the computed LDOS of the $\mathrm{V}=\mathrm{O}$ defect, whereas Au attachment to an $\mathrm{O}$ vacancy does not produce states close to the Fermi level. From a purely energetic point of view, the albeit rare occurrence of Au atoms on vanadyl vacancies is surprising, because the binding energy is $0.78 \mathrm{eV}$ lower than for the regular $\mathrm{V}=\mathrm{O}$ bridge sites. The population of $\mathrm{V}=\mathrm{O}$ defects therefore suggests that the formation of bridge-bonded Au species is connected with a kinetic barrier, which might be related to the distortion of two $\mathrm{V}=\mathrm{O}$ groups in order to accommodate the gold. ${ }^{262}$

The experimental finding that Au shows only a small affinity for the defects in the $\mathrm{V}=\mathrm{O}$ terminated $\mathrm{V}_{2} \mathrm{O}_{3}$ surface is in deviance with the generally accepted importance of defects for adsorption processes on oxide materials. ${ }^{21,27,36}$ In contrast to point defects in highly ionic oxide materials, the $\mathrm{V}=\mathrm{O}$ vacancy does neither expose a chemically unsaturated and hence reactive surface site nor traps excess electrons that could promote the bonding of ad-species. The removal of a single $\mathrm{V}=\mathrm{O}$ group simply provides access to the up-most bulk-like oxygen plane of $\mathrm{V}_{2} \mathrm{O}_{3}$ that does not offer an attractive binding environment for adsorbates either. In this sense, the vanadyl vacancies in $\mathrm{V}_{2} \mathrm{O}_{3}$ thin films are a rather untypical defect type and the results obtained for this system should not be generalized.

The role of surface defects in the adsorption behavior of $\mathrm{V}_{2} \mathrm{O}_{3} / \mathrm{Au}(111)$ has also been investigated for organic molecules, in particular for magnesium phthalocyanine (MgPc). ${ }^{433} \mathrm{~A}$ comparison with the Au experiments discussed above is instructive, because the conjugated MgPc interacts mainly via polarization of its $\pi$ - electronic system, while metal atoms are able to form true chemical bonds. ${ }^{438}$ Upon room temperature deposition onto the $\mathrm{V}=\mathrm{O}$-terminated surface, the cloverleaf shape of the flat-lying MgPc becomes visible in the STM. However, the characteristic four-fold symmetry of the molecule is disturbed in most cases, as one or two molecular legs appear with reduced topographic contrast (Fig. 4.39A). Performing manipulation experiments with the STM tip, it can be shown that the asymmetric appearance of the molecule results from its attachment to a surface defect. For this purpose, the surface is scanned at low bias voltage and small tip-sample distance until a chosen molecule desorbs from the film and the underlying vanadyl lattice becomes visible. Highly symmetric 
molecules, as the one shown in Fig. 4.39B, are found to be located in defect-free oxide regions, as only molecular fragments (e.g. two $\mathrm{H}$ atoms) but no $\mathrm{V}=\mathrm{O}$ vacancy are detected after removal of the adsorbate. Desorption of an asymmetric $\mathrm{MgPc}$, on the other hand, always reveals a defect in the $\mathrm{V}=\mathrm{O}$ lattice underneath, indicating that the reduced molecular symmetry is indeed related to its interaction with the $\mathrm{V}=\mathrm{O}$ vacancy (Fig. 4.39C). As only $10 \%$ of the molecules display an unperturbed symmetry, MgPc adsorption to surface defects seems to be thermodynamically favored.
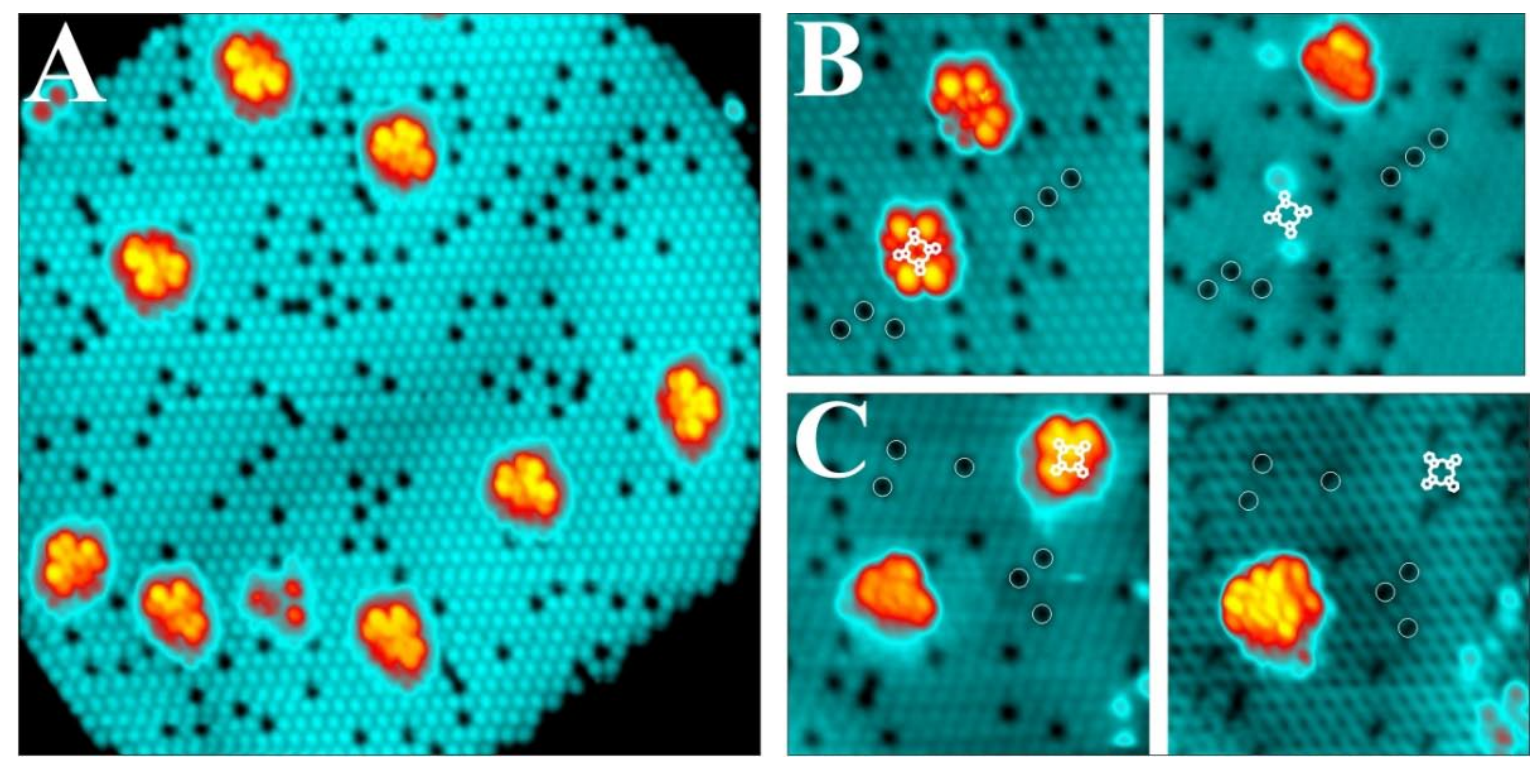

Fig. 4.39:

(A) STM topographic image of a $\mathrm{V}_{2} \mathrm{O}_{3}$ island on $\mathrm{Au}(111)$ after exposure to MgPc molecules at $300 \mathrm{~K}$ $\left(-1.8 \mathrm{~V}, 22 \times 22 \mathrm{~nm}^{2}\right)$. (B) STM image of a defect-bound (top) and a regular MgPc molecule (bottom) $\left(-1.6 \mathrm{~V}, 10 \times 10 \mathrm{~nm}^{2}\right)$ before and after desorption of the latter by scanning the surface at $-0.5 \mathrm{~V}$ sample bias. (C) Desorption of a defect-bound MgPc $\left(-1.6 \mathrm{~V}, 9.5 \times 9.5 \mathrm{~nm}^{2}\right)$. The $\mathrm{V}=\mathrm{O}$ vacancy that was located below the distorted molecular leg is clearly identified in the right image. Circles in (B) and (C) are guides to the eye to recognize identical surface regions. ${ }^{433}$

Two binding contributions have to be considered when analyzing the MgPc interaction with the ideal $\mathrm{V}_{2} \mathrm{O}_{3}$ surface. First, the central $\mathrm{Mg}$ atom, as the positive charge centre of the molecule, 439 experiences a Coulomb attraction towards the negatively-charged vanadyl oxygen. As a result, the centre of the MgPc is always found to be on top of a $\mathrm{V}=\mathrm{O}$ group and never binds to a surface defect. Secondly, the molecular frame interacts via its delocalized $\pi$ electronic system with the $V 3 d$ states in the oxide film. ${ }^{408}$ Polarization forces, as the dominant interaction mechanism, are hereby only efficient for the bulk $\mathrm{V}$ atoms with a partly filled $d$ shell ( $3 d^{2}$ configuration), but negligible for the $\mathrm{V}$ atoms in the $\mathrm{V}=\mathrm{O}$ groups due to their $3 d^{0}$ configuration. ${ }^{26}$ The vanadyl termination can therefore be considered as a chemically inert spacer layer that only decreases the overlap between the molecular $\pi$-system and the oxide 
LDOS. Above a $\mathrm{V}=\mathrm{O}$ vacancy, one leg of the MgPc molecule is able to approach the oxide surface, because the steric repulsion with the vanadyl group is missing. The $\pi$-system of this leg can thus overlap more efficiently with the $V 3 d$ states underneath, leading to an increase of the total binding energy with respect to the regular-bound molecule. The preferred MgPc adsorption to defects in the $\mathrm{V}=\mathrm{O}$ lattice is therefore traced back to a locally reduced moleculeoxide separation at the position of the vacancy site. This conclusion is supported by $\mathrm{dI} / \mathrm{dV}$ spectroscopy performed on regular versus defect-bonded MgPc molecules (not shown). ${ }^{43}$ The similarity of both spectra suggests that no new electronic states are induced in the MgPc upon interaction with the oxide defect; hence no direct chemical bond is formed when the molecule attaches to the $\mathrm{V}=\mathrm{O}$ vacancy.

The adsorption behavior of the more reactive O-defects in $\mathrm{V}=\mathrm{O}$-terminated $\mathrm{V}_{2} \mathrm{O}_{3}(0001)$ but also in $\mathrm{V}_{2} \mathrm{O}_{5}(001)$ has been investigated with respect to water and methanol. ${ }^{440,441}$ In both cases, the dissociation of the molecular species in the presence of O-defects has been concluded from the STM and TDS measurements. While in the case of water, healing of the defect and formation of two $\mathrm{OH}$-groups is revealed, methanol attaches in the form of methoxy $\left(-\mathrm{O}-\mathrm{CH}_{3}\right)$ to the chemically unsaturated $\mathrm{V}$ atom of the O-defect. In contrast, the $\mathrm{V}=\mathrm{O}$ vacancy sites turned out to be non-reactive for these two and similar molecular adsorbates. ${ }^{442}$

The final topic of this paragraph concerns the adsorption characteristics of step edges and line defects in thin oxide films. The outstanding role of surface steps for the nucleation and growth of metal particles has been realized already at the very beginning of STM experiments on oxide surfaces. ${ }^{32,335,443}$ Step edges have been identified as the preferred adsorption sites for weakly bound adsorbates (e.g. Ag, $\mathrm{Pd}, \mathrm{Cu}$ and various molecular species) on structurally homogenous oxide surfaces with a low concentrations of point defects (see Fig. 4.36A and Fig. 4.40A). ${ }^{27,48,95,431}$ Their increased binding potential with respect to terrace sites results from the interplay of different effects: (i) a reduced coordination of the step and corner atoms, (ii) a deviating electronic structure and (iii) a discontinuity in the diffusive material transport due to Ehrlich-Schwöbel barriers at the edge. ${ }^{444}$ The importance of step edges for adsorption can even be enhanced by artificially decreasing the intrinsic oxide-adsorbate interaction, for instance via surface hydroxylation. ${ }^{322}$ 

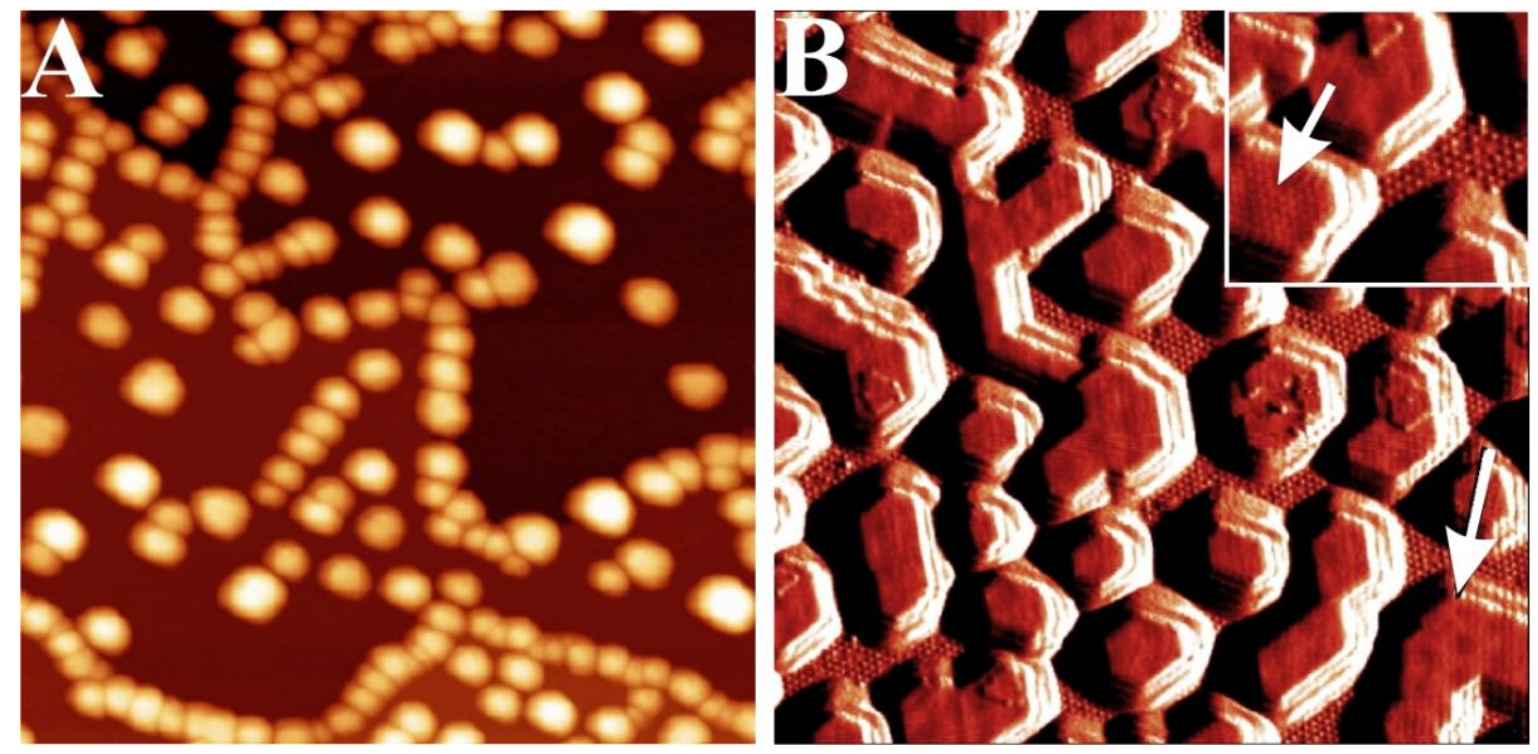

Fig. 4.40: (A) STM image of Pd particles grown on alumina/NiAl(110) $\left(-3.0 \mathrm{~V}, 120 \times 120 \mathrm{~nm}^{2}\right)$. The arrangement of the deposits reflects the preferred Pd nucleation along step edges and dislocation lines in the alumina film. (B) Pt particle on $\mathrm{Fe}_{3} \mathrm{O}_{4} / \mathrm{Pt}(111)$ exposed to $540 \mathrm{~L}$ of $\mathrm{O}_{2}$ at $500 \mathrm{~K}$ and flashed to $850 \mathrm{~K}$ in vacuum $\left(0.5 \mathrm{~V}, 80 \times 80 \mathrm{~nm}^{2}\right)$. The arrow points to a Pt top facet that displays the distinct Pt$\mathrm{Fe}_{3} \mathrm{O}_{4}$ Moiré pattern. The inset shows an atomically resolved Pt particle, which exhibits the same atomic periodicity as the bare oxide surface (arrow). Both observations indicate the growth of a thin $\mathrm{FeO}_{\mathrm{x}}$ layer on-top of the Pt particles due to the high temperature treatment (encapsulation).

Dislocation lines and domain boundaries are the second import class of line defects. Although their formation is directly linked to the growth of oxide films on metal supports (see Chapter 3.4), the distinct adsorption behavior of such defects has not yet been analyzed with the STM at the atomic level. Most of the experiments focused on the nucleation and growth of metal particles along the line defects, but failed to indentify the underlying interaction mechanisms at the local scale. Preferential nucleation of metals at misfit-induced dislocation lines has been revealed for many thin-film systems, for instance for $\mathrm{Pd}$ and $\mathrm{Rh}$ on alumina/ $\mathrm{NiAl}(110),{ }^{48,445}$ for $\mathrm{Co}$ on alumina/CoAl(001) ${ }^{446}$ and for $\mathrm{Au}$ on $\mathrm{MgO} / \mathrm{Mo}(001){ }^{252}$ Even the self-organized growth on nano-particles can be observed, if the structural relaxation of a thin oxide film includes the formation of an ordered dislocation network. Such template effects have been described for the $\mathrm{CoO} / \mathrm{Ag}(001)$ system, ${ }^{211}$ where a buried network of interfacial dislocations triggers the spatial ordering of $\mathrm{Ni}$ ad-particles. ${ }^{210}$ Also a bilayer $\mathrm{TiO}_{\mathrm{x}}$ film $(\mathrm{x} \sim 1)$ grown on $\operatorname{Pt}(111)$ exhibits a well-ordered defect structure that is suitable to induce the self-assembly of $\mathrm{Au}$ clusters into a quasi hexagonal pattern. ${ }^{212}$ The development of regular dislocation networks has been observed for other oxide systems, e.g. for $\mathrm{MgO} / \mathrm{Mo}(001)^{51}$ and $\mathrm{NiO} / \mathrm{Ag}(001)^{142}$, making them potential candidates for a self-organized growth of metal deposits as well. 
The atomistic mechanism that underlies the preferential metal binding to dislocation lines, has been identified only for the alumina/NiAl(110) system so far. ${ }^{195}$ In an extensive experimental screening, the affinity of different metals to bind to the oxide line defects was correlated with their chemical properties. ${ }^{48}$ While Rh, Pd and Co nucleate almost exclusively along the dislocation lines (Fig. 4.40A), $\mathrm{Au}^{387}$ and $\mathrm{Pt}^{443}$ as well as $\mathrm{V}$ and $\mathrm{Al}$ attach to the defect-free oxide terraces with equal probability. DFT calculations revealed the oxygen-deficient nature of the alumina line defects with one $\mathrm{O}$ atom missing per unit cell with respect to the unperturbed oxide lattice. ${ }^{195}$ Simultaneously, a set of unoccupied states is produced in the oxide band gap around the vacancy sites. ${ }^{193}$ Both contributions, the disturbed oxide stoichiometry and the modified electronic structure are responsible for a substantial binding increase along the defect lines, especially for weakly and moderately interacting metals. For example, the adsorption energy of $\mathrm{Pd}$ and $\mathrm{Rh}$ atoms rises by 0.7 and $1.7 \mathrm{eV}$, respectively, rationalizing their large affinity to bind to the misfit dislocations (Fig. 4.40A). ${ }^{195}$ For $\mathrm{Au}$ and $\mathrm{Pt}$, the energy difference between defect and regular binding sites is much smaller, because these electronegative materials are able to activate strong charge-mediated binding mechanisms even on stoichiometric terraces. ${ }^{375}$ Also $\mathrm{V}$ and $\mathrm{Al}$, being metals with notoriously high heats of oxidation, exhibit sufficiently strong metal-oxide interactions to render nucleation on defect-free oxide patches highly probable.

The phenomenon of encapsulation, as discussed for metal particles on bulk $\mathrm{TiO}_{2}$ in Chapter 4.2, does also occur for thin oxide films. For example, Pt particles on a $\mathrm{Fe}_{3} \mathrm{O}_{4} / \mathrm{Pt}(111)$ film coat with a thin oxide layer of unknown composition during tempering to $800 \mathrm{~K}$ (Fig. 4.40B) ${ }^{447}$ Due to the limited thickness of most oxide films, encapsulation is often replaced by the complete immersion of the metal deposits towards the metal-oxide interface and the segregation of the oxide to the surface. As a consequence, the almost unperturbed and particle-free oxide morphology reappears in the STM topographic images after the annealing step. This behavior has been reported for $\mathrm{Pd}$ and Co particles on FeO/Pt(111) ${ }^{168}$ and $\mathrm{VO} / \mathrm{Rh}(111)^{296}$ systems, respectively. It is triggered by a considerable decrease of the surfacefree-energy when going from the particle-covered to the bare oxide surface, and therefore similar to the encapsulation mechanism found for bulk oxides. 


\section{Summary and Outlook}

This review aimed for providing a survey of the enormous potential of scanning tunneling microscopy and embedded spectroscopic techniques to explore the properties of oxide surfaces and their interaction with atomic and molecular adsorbates. In contrast to spatially averaging experimental techniques, the STM is able to probe the surface at the local, preferably atomic scale. This is of particular importance, as properties of real oxides are often governed by structural inhomogeneities, e.g. by defects and low-coordinated sites. The STM plays hereby an outstanding role in resolving the real-space, atomic configuration of oxide materials, providing essential input parameters for the development of structural models. Additionally, conductance spectroscopy with the STM can be employed to probe the occupied and unoccupied state density of oxide surfaces and adsorbate complexes. The capabilities of this technique has been demonstrated at several points of this review, for instance to determine the nature of defect levels in the oxide band gap and to monitor electron quantization effects in metal deposits of different size and shape.

The potential of STM to unravel magnetic, vibrational and optical properties of oxide materials could only be touched in this article and is still far from being exploited. Many transition-metal oxides are anti-ferromagnets and exhibit complex surface magnetic structures that are essentially unknown. ${ }^{166}$ Promising attempts to unravel the local magnetic properties of oxides have been carried out for $\mathrm{Fe}_{3} \mathrm{O}_{4}$ samples using spin-polarized tunneling. ${ }^{265,293}$ Furthermore, oxide are well characterized by their phonon signature that reflects not only the overall crystal structure and stoichiometry, but provides information on the local coordination of the oxide ions as well. ${ }^{151,155,421}$ The possibility to probe oxide phonons via inelastic electron tunneling spectroscopy with the STM could open a new and versatile route to analyze oxide properties at the local scale. Preliminary results along this line have been obtained only for graphite samples so far, ${ }^{448}$ while experiments on oxide materials are already in progress. Finally, optical methods, e.g. UV-VIS spectroscopy, are widely used to gain insights into the oxide electronic structure, for instance into the gap size, the presence of localized defect states and the energy of electron-hole pair excitations. ${ }^{130,133,449}$ Also here, a spatially-resolving technique would be highly desirable, as optical properties of oxide materials are almost exclusively defect-mediated and hence sensitive to the local environment in which the optical transition takes place. $^{23,55}$ Photon emission spectroscopy with the STM, where local optical modes are excited by electron/hole injection from the tip, offers the required spatial resolution. The potential of this technique has already been demonstrated with experiments 
performed on single molecules, ${ }^{124,125}$ metal particles ${ }^{119,134}$ and quantum dots, ${ }^{121}$ while comparable studies on the oxide materials are still scarce. ${ }^{51,82,119}$ Photon emission spectroscopy with the STM will however develop into a versatile tool to explore the local optical properties and their interrelation with the photo-catalytic performance of oxide materials. In general, the exploitation of the spectroscopic capabilities of the STM to investigate oxide surfaces at the atomic level is only at the beginning and substantial progress in this direction can be expected in the near future.

The overwhelming amount of experiments discussed in this review has been performed on thin-film and not on bulk oxides. This experimental approach bears some limitations, because certain bulk properties are not fully restored in thin oxide films supported by metal single crystals. However, thin-film oxides have indubitable advantages in many other aspects, for instance what their large electron transmissibility and the facile preparation of high-quality surfaces concerns. Thin oxide films exhibit another significant benefit, namely the unique possibility to modify their structural, electronic and adsorption properties in a controlled manner. ${ }^{53,367}$ This flexibility enables the creation of a new class of low-dimensional oxide materials, whose specifications are adaptable to the requirements of numerous applications in the material-sciences, in heterogeneous catalysis, nano-electronics and optics.

The key parameters that might be controlled in a thin-film system are the:

- Oxide structure that means the lattice constant, growth direction and surface termination of the oxide film. These parameters are to a large extent determined by the crystallographic and chemical properties of the metal support, but also by the chosen preparation conditions. The template effect of a suitable substrate offers the possibility to stabilize even polar oxide films, making their unusual electronic and chemical properties accessible to the experiment.

- Oxide-support interactions. The interfacial coupling between the metal substrate and the oxide film influences various material parameters, e.g. the gap size, the state-density at the oxide surface, the work function and the built-in electric field. A proper choice of the metal substrate therefore promotes the development of desired oxide properties.

- Defect structure. Defects can be introduced into oxide films in various ways, for instance by adjusting a finite lattice mismatch with the support to enforce plastic deformations, by inserting impurities and dopants during growth, or by treating the film with electrons, ions or thermal cycles. The preparation of the right kind and 
concentration of defects is an effective means to control the electronic, optical and chemical properties of oxide films.

- Surface properties. The dominant adsorption mechanism of an oxide film is not only given by the structure, stoichiometry and termination of its surface, but also by the film thickness and the residual influence of the metal support. The latter enables an exchange of electrons through the oxide spacer, leading to the formation of cationic or anionic adspecies. Their charge state might also be controlled by inserting suitable dopants into the subsurface region. By exploiting these approaches, the adsorption behavior of oxide films can be modified over a wide range in order to fabricate ad-structures with defined geometric and electronic properties. Also the catalytic performance of the metal-oxide system can be improved by this means.

Only some routes towards tailored adsorption systems on the basis of thin oxide films could be addressed in this review, while other possibilities are widely unexplored yet. On all account, oxide thin films on metal supports can be considered as an interesting class of materials with versatile properties and large technological potential. Without doubt, the STM will continue to play a decisive role in exploring the properties of these systems in real-space and with atomic resolution. However, its main field of operation is expected to shift away from pure imaging towards new spectroscopic and atom-manipulation techniques, aiming for the fabrication and analysis of quantum devices with yet unexplored properties. Finally, highspeed STM experiments at elevated temperature and pressure conditions will gain more attention, as they enable the investigation of dynamic processes and chemical reactions with both temporal and spatial resolution.

\section{Acknowledgement}

This article would have been impossible without the collaboration of many people. My special thanks go to H.-J. Freund for his constant support of my work. For countless theoretical insights, I am grateful to G. Pacchioni, M. V. Ganduglia-Pirovano, H. Häkkinen and L. Giordano. However, the real brunt of this work was borne by the $\mathrm{PhD}$ students and post-docs in my group, in particular by H. M. Benia, J. F. Jerratsch, X. Lin, Ph. Myrach, E. D. L. Rienks, V. Simic-Milosevic and S. Ulrich, and my coworkers in the department, M. Heyde, Th. Risse, H.-P. Rust, and M. Sterrer. My deepest gratitude goes to all of them. For financial support, I am grateful to the Deutsche Forschungsgemeinschaft and the COST Action D41. 


\section{List of References}

${ }^{1}$ P. A. Schweitzer, Fundamentals of metallic corrosion: atmospheric and media corrosion of metals, 2nd Ed. (Taylor \& Francis, Boca Raton, 2007).

2 D. A. Jones, Principles and prevention of corrosion, (Prentice Hall, Upper Saddle River, 1995).

${ }^{3}$ K. J. Bachmann, The materials science of microelectronics, (VCH, New York, 1995).

${ }^{4}$ A. I. Kingon, J. P. Maria, S. K. Streiffer, Nature 406 (2000) 1032.

${ }^{5}$ G. Ertl, H. Knözinger, F. Schueth, J. Weitkamp [Eds.], Handbook of heterogeneous catalysis, $2^{\text {nd }}$ edition, (Wiley-VCH, Weinheim, 2008).

${ }^{6}$ A. Trovarelli, Catal. Rev. Sci. Eng. 38 (1996) 439.

${ }^{7}$ W. C. Conner, J. L. Falconer, Chem. Rev. 95 (1995) 759.

${ }^{8}$ E. Iglesia, Appl. Catal. A 161 (1997) 59.

${ }^{9}$ B. Yoon, H. Hakkinen, U. Landman, A. S. Worz, J. M Antonietti, S. Abbet, K. Judai, U. Heiz, Science 307 (2005) 403.

${ }^{10}$ M.S. Chen, D.W. Goodman, Science 306 (2004) 252.

${ }^{11}$ A. Khodakov, B. Olthof, A. T. Bell, E. Iglesia, J. Catal. 181 (1999) 205.

${ }^{12}$ B. L. Min, W. T. Wallace, D. W. Goodman, J. Phys. Chem. B 108 (2004) 14609; W. T. Wallace, B. K. Min, D. W. Goodman, J. Mol. Catal. A 228 (2005) 3.

${ }^{13}$ M. Imada, A. Fujimori, Y. Tokura, Rev. Mod. Phys. 70 (1998) 1039.

${ }^{14}$ Q. Wan, Q. H. Li, Y. J. Chen, T. H. Wang, X. L. He, C. L. Lin, Appl. Phys. Lett. 84 (2004) 3654.

${ }^{15}$ B. O’Regan, M. Grätzel, Nature 353 (1991) 737.

${ }^{16}$ D.E. Ollis, H.Al-Ekabi [Eds.], Photo-catalytic treatment of water and air, (Elsevier, Amsterdam, 1993).

${ }^{17}$ A. Hamnett, J. B. Goodenough, Binary transiton metal oxides, (Springer, Berlin, 1984).

${ }^{18}$ P. A. Cox, Transition metal oxides, (Clarendon Pr., Oxford, 1992).

${ }^{19}$ V. E. Henrich, P. A. Cox, The Surface Science of Metal Oxides, p. 247, (University Press, Cambridge, 1994).

${ }^{20}$ H.-J. Freund, Faraday Disc. 114 (2000) 1.

${ }^{21}$ G. Pacchioni, Sol. Stat. Science 2 (2000) 161.

${ }^{22}$ R. J. D. Tilley, Defects in solids, (Wiley, Hoboken, 2008).

${ }^{23}$ A. L. Shluger, P. V. Sushko, and L. N. Kantorovich, Phys. Rev. B 59 (1999) 2417.

${ }^{24}$ G. H. Rosenblatt, M.W. Rowe, G. P. Williams, R.T. Williams, Y. Chen, Phys. Rev. B 39 (1989) 10309.

${ }^{25}$ J. A. Wingrave, [Ed.], Oxide Surfaces, (Dekker, New York, 2001).

${ }^{26}$ G. Kresse, S. Surnev, J. Schoiswohl, F. P. Netzer, Surf. Sci. 555 (2004) 118.

${ }^{27}$ C. T. Campbell, Surf. Sci. Rep. 27 (1997) 1.

${ }^{28}$ W. Weiss, W. Ranke, Prog. Surf. Sci. 70 (2002) 1.

${ }^{29}$ S. Surnev, M. G. Ramsey, F. P. Netzer, Progr. Surf. Sci. 73 (2003) 117.

${ }^{30}$ A. Wander, I. J. Bush, N. M. Harrison, Phys. Rev. B 68 (2003) 233405.

${ }^{31}$ G. Kresse, M. Schmid, E. Napetschnig, M. Shishkin, L. Köhler, P. Varga, Science 308 (2005) 1440.

${ }^{32}$ H.-J. Freund, B. Dillmann, D. Ehrlich, M. Hassel, R.M. Jäger, H. Kuhlenbeck, C.A. Ventrice, F. Winkelmann, S. Wohlrab, C. Xu, T. Bertrams, A. Brodde, H. Neddermeyer, J. Mol. Catal. 82 (1993) 143.

${ }^{33}$ C. Noguera, Physics and chemistry at oxide surfaces, (University Press, Cambridge, 1996)

${ }^{34}$ A. Barbier, C. Mocuta, H. Kuhlenbeck, K. F. Peters, B. Richter, G. Renaud, Phys. Rev. Lett. 84 (2000) 2897.

${ }^{35}$ Y. Wang, B. Meyer, X. Yin, M. Kunat, D. Langenberg, F. Träger, A. Birkner, C. Wöll, Phys. Rev. Lett. 95 (2005) 266104.

${ }^{36}$ H.-J. Freund, E. Umbach [Eds.], Adsorption on ordered surfaces of ionic solids and thin films (Springer, Berlin, 1993).

${ }^{37}$ C. R. Henry, Surf. Sci. Rep. 31 (1998) 235.

${ }^{38}$ G. A. Somorjai, J. Y. Park, Chem. Soc. Rev. 37 (2008) 2155.

${ }^{39}$ T. Brandstetter, M. Draxler, M. Hohage, P. Zeppenfeld, Phys. Rev. B 78 (2008) 075402. 
${ }^{40}$ U. Diebold, Surf. Sci. Rep. 48 (2003) 53.

${ }^{41}$ U. Ozgur, Y. I. Alivov, C. Liu, A. Teke, M. A. Reshchikov, S. Dogan, V. Avrutin, S. J. Cho, H. Morkoc, J. Appl. Phys. 98 (2005) 041301.

${ }^{42}$ M. Li, W. Hebenstreit, L. Gross, U. Diebold, M. Henderson, D.R. Jennison, P. Schultz, M. Sears, Surf. Sci. 437 (1999) 173.

${ }^{43}$ J. M. Pan, B. L. Maschhoff, U. Diebold, T. E. Madey, J. Vac. Sci. Technol. A 10 (1992) 2470.

${ }^{44}$ S. Wendt, R. Schaub, J. Matthiesen, E. K. Vestergaard, E. Wahlstrom, M. D. Rasmussen, P. Thostrup, L. M. Molina, E. Laegsgaard, I. Stensgaard, B. Hammer, F. Besenbacher, Surf. Sci. 598 (2005) 226.

${ }^{45}$ D. W. Goodman, Surf. Rev. Lett. 2 (1995) 9; D. W. Goodman, Chem. Rev. 95 (1995) 523.

${ }^{46}$ S. C. Street, C. Xu, D. W. Goodman, Ann. Rev. Phys. Chem. 48 (1997) 43.

${ }^{47}$ S. A. Chambers, Surf. Sci. Rep. 39 (2000) 105.

${ }^{48}$ M. Bäumer, H.-J. Freund, Progr. Surf. Sci. 61 (1999) 127.

${ }^{49}$ H.-J. Freund, M. Bäumer, J. Libuda, T. Risse, G. Rupprechter, S. Shaikhutdinov, J. Catal. 216 (2003) 223.

${ }^{50}$ C. T. Campbell, Phys. Rev. Lett. 96 (2006) 066106.

${ }^{51}$ S. Benedetti, H. M. Benia, N. Nilius, S. Valeri, H.-J. Freund, Chem. Phys. Lett. 430 (2006) 330.

${ }^{52}$ J. Libuda, H.-J. Freund, Surf. Sci. Rep. 57 (2005) 157.

${ }^{53}$ H.-J. Freund, Surf. Sci. 500 (2002) 271; H. J. Freund, Surf. Sci. 601 (2007) 1438.

${ }^{54}$ J. T. Yates, Experimental innovations in surface science, (AIP Press, New York, 1998).

${ }^{55}$ P. V. Sushko, A. L. Shluger, C. R. Catlow, Surf. Sci. 450 (2000) 153.

${ }^{56}$ H. Haberland [Ed.], Clusters of atoms and molecules, (Springer, Berlin, 1994).

${ }^{57}$ H. Hövel, I. Barke, Prog. Surf. Sci. 81 (2006) 53.

${ }^{58}$ U. Kreibig, W. Vollmer, Optical Properties of Metal Clusters, Springer Series Materials Science, Vol. 25, (Springer, Berlin, 1995).

${ }^{59}$ R. Wiesendanger, Scanning probe microscopy and spectroscopy, (Univ. press, Cambridge, 1994).

${ }^{60}$ J. C. Chen, Introduction to scanning tunneling microscopy, (Oxford Univ. Press, New York, 1993).

${ }^{61}$ R. M. Overney, H. Häfke, E. Meyer, H. J. Güntherodt, Surf. Sci. 277 (1992) L29.

${ }^{62}$ Y. Namai, K. I. Fukui, Y. Iwasawa, Catal. Tod. 85 (2003) 79.

${ }^{63}$ C. Barth, C. R. Henry, Phys. Rev. Lett. 91 (2003) 196102.

${ }^{64}$ C. Barth, M. Reichling, Nature 414 (2001) 54.

${ }^{65}$ G. Hamm, C. Barth, C. Becker, K. Wandelt, C. R. Henry, Phys. Rev. Lett. 97 (2006) 126106.

${ }^{66}$ M. Heyde, M. Sterrer, H.-P. Rust, H.-J. Freund, Appl. Phys. Lett. 87 (2005) 083104.

${ }^{67}$ M. Heyde, M. Sterrer, G. Simon, H.-P. Rust, H.-J. Freund, Appl. Phys. Lett. 89 (2006) 263107.

${ }^{68}$ J. Tersoff, D. R. Hamann, Phys. Rev. Lett. 50 (1938) 1998.

69 J. Bardeen, Phys. Rev. Lett. 6 (1961) 57.

${ }^{70}$ J. Tersoff, D. R. Hamann, Phys. Rev. Lett. 50 (1938) 1998.

${ }^{71}$ R. J. Hamers, Annu. Rev. Phys. Chem. 40 (1989) 531.

${ }^{72}$ D. A. Bonnell, Prog. Surf. Sci. 57 (1998) 187.

${ }^{73}$ K. H. Gundlach, Sol. Stat. Electron. 9 (1966) 949.

${ }^{74}$ G. Binnig, H. Rohrer, C. Gerber, E. Weibl, Appl. Phys. Lett. 40 (1982) 178.

${ }^{75}$ M. R. Castell, P. L. Wincott, N. G. Condon, C. Muggelberg, G. Thornton, S. L. Dudarev, A. P. Sutton, G. A. D. Briggs, Phys. Rev. B 55 (1997) 7859.

${ }^{76}$ F. Esch, S. Fabris, L. Zhou, T. Montini, C. Africh, P. Fornasiero, G. Comelli, R. Rosei, Science 309 (2005) 752.

${ }^{77}$ M. Sterrer, M. Heyde, M. Nowicki, N. Nilius, T. Risse, H.-P. Rust, G. Pacchioni, H.-J. Freund, J. Phys. Chem. B 110 (2006) 46.

${ }^{78}$ T. Bertrams, A. Brodde, H. Neddermeyer, J. Vac. Sci. Technol. B 12 (1994) 2122.

${ }^{79}$ I. Sebastian, H. Neddermeyer, Surf. Sci. 454 (2000) 771.

${ }^{80}$ K. H. Hansen, T. Worren, E. Laegsgaard, F. Besenbacher, I. Stensgaard, Surf. Sci. 475 (2001) 96.

${ }^{81}$ K. Meinel, A. Eichler, K.-M. Schindler, H. Neddermeyer, Surf. Sci. 562 (2004) 204.

${ }^{82}$ H. M. Benia, P. Myrach, N. Nilius, New J. Phys. 10 (2008) 013010. 
${ }^{83}$ S. Datta, W. Tian, S. Hong, R. Reifenberger, J. I. Henderson, C. P. Kubiak, Phys. Rev. Lett. 79 (1997) 2530.

${ }^{84}$ S. W. Wu, G. V. Nazin, X. Chen, X. H. Qui, W. Ho, Phys. Rev. Lett. 93 (2004) 236802.

${ }^{85}$ R. M. Feenstra, Phys. Rev. B. 50, 4561 (1994), G. J. de Raad, D. M. Bruls, P. M. Koenraad, J. H. Wolter, Phys. Rev. B 66 (2002) 195306.

${ }^{86}$ M.-C. Blüm, M. Pivetta, F. Patthey, W.-D. Schneider, Phys. Rev. B 73 (2006) 195409.

${ }^{87}$ G. V. Nazin, X. H. Qiu, W. Ho, Phys. Rev. Lett. 95 (2005) 166103; G. V. Nazin, X. H. Qui, W. Ho, J. Chem. Phys. 122 (2005) 181105.

${ }^{88}$ J. Repp, G. Meyer, F. E. Olson, M. Persson, Science 305 (2004) 493.

${ }^{89}$ A.E. Hanna, M. Tinkham, Phys. Rev. B 44 (1991) 5919.

${ }^{90}$ M. Amman, S. F. Field, R. C. Jaklevic, Phys. Rev. B 48 (1993) 12104.

${ }^{91}$ J. Hou, B. Wang, J. Yang, X. Wang, H. Q. Wang, Q. Zhu, X. Xiao, Phys. Rev. Lett. 86 (2001) 5321.

${ }^{92}$ R. G. C. Moore, S. D. Evans, T. Shen, C. E. Hodson, Physica E 9 (2001) 253.

${ }^{93}$ M. Amman, R. Wilkes, E. Ben-Jacob, P. D. Maker, R. C. Jaklevic, Phys. Rev. B 43 (1991) 1146.

${ }^{94}$ C. Kittel, Introduction to Solid State Physics, (Wiley, New York, 1996).

${ }^{95}$ N. Nilius, M. Kulawik, H.-P. Rust, H.-J. Freund, Surf. Sci. 572 (2004) 347.

${ }^{96}$ K. Sattler, Z. Physik D 19 (1991) 287.

${ }^{97}$ G. Binnig, K. H. Frank, H. Fuchs, N. Garcia, B. Reihl, H. Rohrer, F. Salvan, A. R. Williams, Phys. Rev. Lett. 55 (1985) 991.

${ }^{98}$ B. Koslowski, C. Dietrich, A. Tschetschetkin, P. Ziemann, Phys. Rev. B 75 (2007) 035421.

${ }^{99}$ L. Jurczyszyn, B. Stankiewicz, Vacuum 54 (1999) 131.

${ }^{100}$ N. Nilius, T. M. Wallis, W. Ho, Science 297 (2002) 1853.

${ }^{101}$ R. Wiesendanger, H. J. Güntherodt, R. J. Gambino, R. Ruf, Phys. Rev. Lett. 65 (1990) 247.

${ }^{102}$ R. Wiesendanger, Curr. Opin. Sol. Stat. Mat. Science 4 (1999) 435.

${ }^{103}$ J. Repp, G. Meyer, Phys. Rev. Lett. 94 (2005) 026803, J. Repp, G. Meyer, S. Paavilainen, F. E. Olsson, M. Persson, Science 312 (2006) 1196.

${ }^{104}$ N. Nilius, T. M. Wallis, W. Ho, J. Phys. Chem. B 109 (2005) 20657.

${ }^{105}$ L. J. Lauhon, W. Ho, Rev. Sci. Instr. 72 (2001) 216; A. Wachowiak, PhD Thesis, (University Hamburg, 2003).

${ }^{106}$ J. Kliewer, R. Berndt, E. V. Chulkov, V. M. Silkin, P. M. Enchenique, S. Crampin, Science 288 (2000) 1399.

${ }^{107}$ N. Nilius, T. M. Wallis, M. Persson, W. Ho, Phys. Rev. Lett. 90 (2003) 196103.

${ }^{108}$ B. C. Stipe, M. A. Rezaei, W. Ho, Science 280 (1998) 1732.

${ }^{109}$ W. Ho, J. Chem. Phys. 117 (2002) 11033.

${ }^{110}$ R. Berndt, in: R. Wiesendanger (Ed.), Scanning Probe Microscopy, Springer Series Nanoscience and Technology,p. 97, (Springer, Berlin, 1998).

${ }^{111}$ A. J. Heinrich, J. A. Gupta, C. P. Lutz, D. M. Eigler, Science 306 (2004) 466.

${ }^{112}$ C. L. Gao, A. Ernst, G. Fischer, W. Hergert, P. Bruno, W. Wulfhekel, J. Kirschner, Phys. Rev. Lett. 101 (2008) 167201.

${ }^{113}$ N. Lorente, M. Persson, Phys. Rev. Lett. 85 (2000) 2997.

${ }^{114}$ J. R. Hahn, H. J. Lee, W. Ho, Phys. Rev. Lett. 85 (2000) 1914.

${ }^{115}$ X. H. Qui, G.V. Nazin, W. Ho, Phys. Rev. Lett. 92 (2004) 206102.

${ }^{116}$ N. Ogawa, G. Mikaelian, W. Ho, Phys. Rev. Lett. 98 (2007) 166103.

${ }^{117}$ N. Liu, N. A. Pradhan, W. Ho, J. Chem. Phys. 120 (2004) 11371.

${ }_{118}$ R. Berndt, J. K. Gimzewski, P. Johansson, Phys. Rev. Let. 71 (1993) 3493.

${ }^{119}$ N. Nilius, N. Ernst, H.-J. Freund, Phys. Rev. Lett. 84 (2000) 3994.

${ }^{120}$ R. Berndt, J. K. Gimzewski, Phys. Rev. B 45 (1992) 14095.

${ }^{121}$ U. Hakanson, M. K. Johansson, M. Holm, C. Pryor, L. Samuelson, W. Seifert, Appl. Phys. Lett. 81 (2002) 4443.

${ }^{122}$ M. Sakurai, C. Thirstrup, M. Aono, Phys. Rev. Lett. 93 (2004) 046102.

${ }^{123}$ G. Hoffmann, J. Kliewer, R. Berndt, Phys. Rev. Lett. 87 (2001) 176803.

${ }^{124}$ X. H. Qui, G. V. Nazin, W. Ho, Science 299 (2003) 542. 
${ }^{125}$ E. Cavar, M.-C. Blüm, M. Pivetta, F. Patthey, M. Chergui, W. D. Schneider, Phys. Rev. Lett. 95 (2005) 196102.

${ }^{126}$ P. Johansson, R. Monreal, Z. Phys. B 84 (1992) 269; P. Johansson, R. Monreal, P. Apell, Phys. Rev. B 42 (1990) 9210.

${ }^{127}$ A. Downes, M. E. Taylor, M. E. Welland, Phys. Rev. B 57 (1998) 6706.

${ }^{128}$ S. Wu, D. L. Mills, Phys. Rev. B 65 (2002) 205420.

${ }^{129}$ B. Pettinger, Surface-Enhanced Raman Scattering - Physics and Applications, Topics Appl. Phys. 103 (2006) 217.

${ }^{130}$ I. Rashba, M. D. Sturge [Eds.], Excitons, (North-Holland Publ., Amsterdam, 1982); K. S. Song, R. T. Williams, Self-trapped excitons, (Springer, Berlin, 1993).

${ }^{131}$ M. Anpo, Y. Yamada, Y. Kubokawa, S. Coluccia, A. Zecchina, M. Che, J. Chem. Soc., Faraday Trans. 184 (1988) 751.

${ }^{132}$ S. Stankic, M. Müller, O. Diwald, M. Sterrer, E. Knözinger, J. Bernardi, Angew. Chem. Int. Ed. 44 (2005) 4917.

${ }^{133}$ R. Hacquart, J.-M. Krafft, G. Costentin, J. Jupille, Surf. Sci. 595 (2005) 172.

${ }^{134}$ N. Nilius, N. Ernst, H.-J. Freund, Surf. Sci. 478 (2001) L327.

${ }^{135}$ N. Nilius, PhD Thesis, (Humboldt University Berlin, 2001).

${ }^{136}$ N. Nilius, N. Ernst, H.-J. Freund, Phys. Rev. B 65 (2002) 115421.

${ }^{137}$ W. Benten, N. Nilius, N. Ernst, H.-J. Freund, Phys. Rev. B 72 (2005) 045403.

${ }^{138}$ A. Liebsch, Phys. Rev. B 48 (1993) 11317.

${ }^{139}$ K. Watanabe, D. Menzel, N. Nilius, H.-J. Freund, Chem. Rev. 106 (2006) 4301.

${ }^{140}$ F. Muller, R. de Masi, P. Steiner, D. Reinicke, M. Stadtfeld, S. Hüfner, Surf. Sci. 459 (2000) 161.

${ }^{141}$ P. Luches, S. Altieri, C. Giovanardi, T. S. Moia, S. Valeri, F. Bruno, L. Floreano, A. Morgante, A. Santaniello, A. Verdini, R. Gotter, T. Hibma, Thin Sol. Films 400 (2001) 139.

142 J. Wollschläger, D. Erdos, H. Goldbach, R. Hopken, K. M. Schröder, Thin Sol. Films 400 (2001) 1.

${ }^{143}$ S. Schintke, S. Messerli, M. Pivetta, F. Patthey, L. Libjoulle, M. Stengel, A. de Vita, W. D. Schneider, Phys. Rev. Lett. 87 (2001) 276801.

${ }^{144}$ A. Ferrari, S. Casassa, C. Pisani, S. Altieri, A. Rota, S. Valeri, Surf. Sci. 588 (2005) 160.

${ }^{145}$ M. C. Gallagher, M. S. Fyfiled, L. A. Bumm, J. P. Cowin, S. A. Joyce, Thin Sol. Films 445 (2003) 90.

${ }^{146}$ G. H. Vurens, V. Maurice, M. Salmeron, G. A. Somorjai, Surf. Sci. 268 (1992) 170.

${ }^{147}$ M. Ritter, W. Ranke, W. Weiss, Phys. Rev. B 57 (1998) 7240.

${ }^{148}$ M. Kiguchi, S. Entani, K. Saiki, T. Goto, A. Koma, Phys. Rev. B 68 (2003) 115402.

${ }^{149}$ R. Arita, Y. Tanida, S. Entani, M. Kiguchi, K. Saiki, H. Aoki, Phys. Rev. B 69 (2004) 235423.

${ }^{150}$ C. Hagendorf, R. Shantyr, H. Neddermeyer, W. Widdra, PhysChemChemPhys 8 (2006) 1575.

${ }^{151}$ C. Hagendorf, S. Sachert, B. Bochmann, K. Kostov, W. Widdra, Phys. Rev. B 77 (2008) 075406.

${ }^{152}$ S. Benedetti, P. Torelli, S. Valeri, H. M. Benia, N. Nilius, G. Renaud, Phys. Rev. B 78 (2008) 195411.

${ }^{153}$ V. Maurice, S. Cadot, P. Marcus, Surf. Sci. 458 (2000) 195.

${ }^{154}$ S. Hildebrandt, C. Hagendorf, T. Döge, C. Jeckstiess, R. Kulla, H. Neddermeyer, T. Uttich, J. Vac. Sci. Technol. A 18 (2000) 1010.

${ }^{155}$ R. M. Jäger, H. Kuhlenbeck, H.-J. Freund, M. Wuttig, W. Hoffmann, R. Franchy, H. Ibach, Surf. Sci. 259 (1991) 235.

${ }^{156}$ V. Podgursky, V. Rose, J. Costina, R. Franchy, Surf. Sci. 601 (2007) 3315; E. Napetschnig, M. Schmid, P. Varga, Surf. Sci. 602 (2008) 1750.

${ }^{157}$ J. M. Gainesa, P. J. Bloemenb, J. T. Kohlheppb, C. W. Bulle-Lieuwmaa, R. M. Wolfa, A. Reindersa, R. M. Jungbluta, P. A. van der Heijdenb, J. T. van Eemerena, J. aan de Steggea, W. J. M. de Jonge, Surf. Sci. 373 (1997) 85.

${ }^{158}$ S.A. Chambers, J.R. Williams, M.A. Henderson, A.G. Joly, M. Varela, S.J. Pennycook, Surf. Sci. 587 (2005) L197.

${ }^{159}$ E. Lundgren, G. Kresse, C. Klein, M. Borg, J. N. Andersen, M. De Santis, Y. Gauthier, C. Konvicka, M. Schmid, P. Varga, Phys. Rev. Lett. 88 (2002) 246103. 
${ }^{160}$ C. Becker, J. Kandler, H. Raaf, R. Linke, T. Pelster, M. Drager, M. Tanemura, K. Wandelt, J. Vac. Sci. Technol. A 16 (1998) 1000.

${ }^{161}$ R. P. Blum, D. Ahlbehrendt, H. Niehus, Surf. Sci. 396 (1998) 176.

162 J. Goniakowski, C. Noguera, Interface Sci. 12 (2004) 93.

${ }^{163}$ S. Benedetti, P. Luches, M. Liberati, S. Valeri, Surf. Sci. 572 (2004) L348.

${ }^{164}$ L. Giordano, J. Goniakowski, G. Pacchioni, Phys. Rev. B 67 (2003) 045410.

${ }^{165}$ L. Giordano, M. Baistrocchi, G. Pacchioni, Phys. Rev. B 72 (2005) 115403.

${ }^{166}$ L. Giordano, G. Pacchioni, J. Goniakowski, N. Nilius, E. D. L. Rienks, H.-J. Freund, Phys. Rev. B 76 (2007) 075416.

${ }^{167}$ T. Orzali, S. Agnoli, M. Sambi, G. Granozzi, Surf. Sci. 569 (2004) 105.

${ }^{168}$ S. Shaikhutdinov, R. Meyer, D. Lahav, M. Bäumer, T. Klüner, H.-J. Freund, Phys. Rev. Lett. 91 (2003) 076102.

${ }^{169}$ C. Lamberti, E. Groppo, C. Prestipino, S. Cassassa, A. M. Ferrari, C. Pisani, C. Giovanardi, P. Luches, S. Valeri, F. Boscherini, Phys. Rev. Lett. 91 (2003) 46101.

${ }^{170}$ Y. D. Kim, T. Wei, D. W. Goodman, Langmuir 19 (2003) 354; E. Ozensoy, B. K. Min, A. K. Santra, D. W. Goodman, J. Phys. Chem. B 108 (2004) 4351.

${ }^{171}$ C. Dri, C. Africh, F. Esch, G. Comelli, O. Dubay, L. Köhler, F. Mittendorfer, G. Kresse, P. Dudin, M. Kiskinova, J. Chem. Phys. 125 (2006) 094701.

${ }^{172}$ Y. J. Kim, C. Westphal, R. X. Ynzunza, H. C. Galloway, M. Salmeron, M. A. van Hove, C. S. Fadley, Phys. Rev. B 55 (1997) R13448.

173 J. Goniakowski, C. Noguera, Phys. Rev. B 79 (2009) 155433.

${ }^{174}$ H. C. Galloway, P. Sautet, M. Salmeron, Phys. Rev. B 54 (1996) 11145.

${ }^{175}$ A. Rosenhahn, J. Schneider, C. Becker, K. Wandelt, Appl. Surf. Sci. 142 (1999) 169.

${ }^{176}$ M. Schmid, G. Kresse, A. Buchsbaum, E. Napetschnig, S. Gritschneder, M. Reichling, P. Varga, Phys. Rev. Lett. 99 (2007) 196104.

177 J. Libuda, F. Winkelmann, M. Bäumer, H.-J. Freund, T. Betrams, H. Neddermeyer, K. Müller, Surf. Sci. 318 (1994) 61.

${ }^{178}$ M. Kulawik, N. Nilius, H.-P. Rust, H.-J. Freund, Phys. Rev. Lett. 91 (2003) 256101.

${ }^{179}$ A. Stierle, F. Renner, R. Streitel, H. Dosch, W. Drube, B. C. Cowie, Science 303 (2004) 1652.

${ }^{180}$ H. Gabasch, W. Unterberger, K. Hayek, B. Klötzer, G. Kresse, C. Klein, M. Schmid, P.Varga, Surf. Sci. 600 (2006) 205.

${ }^{181}$ F. Sedona, G. A. Rizzi, S. Agnoli, F. X. Llabrés i Xamena, A. Papageorgiou, D. Ostermann, M. Sambi, P. Finetti, K. Schierbaum, G. Granozzi, J. Phys. Chem. B 109 (2005) 24411.

${ }^{182}$ F. Sedona, S. Agnoli, G. Granozzi, J. Phys. Chem. B 110 (2006) 15359.

${ }^{183}$ G. Bordier, C. Noguera, Phys. Rev. B 44 (1991) 6361.

${ }^{184}$ W. Mönch, Surf. Sci. 299 (1994) 928.

${ }^{185}$ M. Kiguchi, R. Arita, G. Yoshikawa, Y. Tanida, M. Katayama, K. Saiki, A. Koma, H. Aoki, Phys. Rev. Lett. 90 (2003) 196803.

${ }^{186}$ G. Pacchioni, L. Giordano, M. Baistrocchi, Phys. Rev. Lett. 94 (2005) 226104.

${ }^{187}$ L. Giordano, F. Cinquini, G. Pacchioni, Phys. Rev. B 73 (2006) 045414.

${ }^{188}$ H. M. Benia, N. Nilius, H.-J. Freund, Surf. Sci. Lett. 601 (2007) L55.

${ }^{189}$ T. König, G. Simon, M. Heyde, H.-J. Freund, J. Phys. Chem. C 113 (2009) 11301.

${ }^{190}$ E. D. L. Rienks, N. Nilius, H.-P. Rust, H.-J. Freund, Phys. Rev. B 71 (2005) 241404.

${ }^{191}$ K. D. Schierbaum, U. K. Kirner, J. F. Geiger, W. Göpel, Sens. Actuat. Chem. 4 (1991) 87.

${ }^{192}$ S. Ulrich, PhD Thesis, (Technical University Berlin, 2009).

${ }^{193}$ N. Nilius, M. Kulawik, H.-P. Rust, H.-J. Freund, Phys. Rev. B 69 (2004) 121401.

${ }^{194}$ V. Simic-Milosevic, N. Nilius, H.-P. Rust, H.-J. Freund, Phys. Rev. B 77 (2008) 125112.

${ }^{195}$ M. Schmid, M. Shishkin, G. Kresse, E. Napetschnig, P. Varga, M. Kulawik, N. Nilius, H.-P. Rust, H.-J. Freund, Phys. Rev. Lett. 97 (2006) 046101.

${ }^{196}$ L. Eierdal, F. Besenbacher, E. Lzegsgaard, I. Stensgaard, Surf. Sci. 312 (1994) 31.

${ }^{197}$ S. Ulrich, N. Nilius, H.-J. Freund, Surf. Sci. 601 (2007) 4603.

${ }^{198}$ I. Sebastian, M. Heiler, K. Meinel, H. Neddermeyer, Appl. Phys. A 66 (1998) S525. 
${ }^{199}$ C. Hagendorf, R. Shantyr, K. Meinel, K.-M. Schindler, H. Neddermeyer, Surf. Sci. 532 (2003) 346.

${ }^{200}$ I. V. Markov, Crystal Growth for Beginners, (World Scientific, Singapore, 2003).

${ }^{201}$ J. R. Willis, S. C. Jain, R. Bullough, Philosoph. Mag. A 62 (1990) 115.

${ }^{202}$ M. Dynna, T. Okada, G. C. Weatherly, Acta Metall. Material. 42 (1994) 1661.

${ }^{203}$ L. B. Freund, J. Appl. Phys. 68 (1990) 2073.

${ }^{204}$ H. Brune, M. Giovannini, K. Bromann, K. Kern, Nature 394 (1998) 451.

${ }^{205}$ M. Horn v. Hoegen, M. Pook, A. Alfalou, B. H. Muller, M. Henzler, Surf. Sci. 284 (1993) 53; M. Horn v. Hoegen, A. Alfalou, H. Pietsch, B. H. Muller, M. Henzler, Surf. Sci. 298 (1993) 29.

${ }^{206}$ J. Schoiswohl, W. Zheng, S. Surnev, M. G. Ramsey, G. Granozzi, S. Agnoli, F. P. Netzer, Surf. Sci. 600 (2006) 1099.

${ }^{207}$ M. Klaua, D. Ullmann, J. Barthel, W. Wulfhekel, J. Kirschner, R. Urban, T. L. Monchesky, A. Enders, J. F. Cochran, B. Heinrich, Phys. Rev. B 64 (2001) 134411.

${ }^{208}$ J. L. Vassent, M. Dynna, A. Marty, B. Gilles, G. Patrat, J. Appl. Phys. 80 (1996) 5727.

${ }^{209}$ G. Renaud, P. Guénard, A. Barbier, Phys. Rev. B 58 (1998) 7310.

${ }^{210}$ P. Torelli, E. A. Soares, G. Renaud, S. Valeri, X. X. Guo, P. Luches, Surf. Sci. 601 (2007) 2651.

${ }^{211}$ P. Torelli, E. A. Soares, G. Renaud, L. Gragnaniello, S. Valeri, X. X. Guo, P. Luches, Phys. Rev. B 77 (2008) 081409.

${ }^{212}$ F. Sedona, S. Agnoli, M. Fanetti, I. Kholmanov, E. Cavaliere, L. Gavioli, G. Granozzi, J. Phys. Chem. C 111 (2007) 8024.

213 J. P. Hirth, J. Lothe, Theory of Dislocations, (McGraw-Hill, New York, 1982).

${ }^{214}$ M. Henzler, W. Göpel, Oberflächenphysik des Festkörpers, (Teubner, Stuttgart, 1994).

${ }^{215}$ M. Bäumer, D. Cappus, H. Kuhlenbeck, H.-J. Freund, G. Wilhelmi, A. Brodde, H. Neddermeyer, Surf. Sci. 253 (1991) 116.

${ }^{216}$ G. Renaud, A. Barbier, O. Robach, Phys. Rev. B 60 (1999) 5872.

${ }^{217}$ J. G. Yoon, H. K. Oh, S. J. Lee, Phys. Rev. B 60 (1999) 2839.

${ }^{218}$ J. Goniakowski, C. Noguera, L. Giordano, Phys. Rev. Lett. 93 (2004) 215702.

${ }^{219}$ C. Tegenkamp, H. Pfnür, W. Ernst, U. Malaske, J. Wollschläger, D. Peterka, K. M. Schröder, V. Zielasek, M. Henzler, J. Phys. Condens. Matter 11 (1999) 9943.

${ }^{220}$ I. Sebastian, T. Bertrams, K. Meinel, H. Neddermeyer, Farad. Discuss. 114 (1999) 129.

${ }^{221}$ T. K. Todorova, M. Sierka, J. Sauer, S. Kaya, J. Weissenrieder, J.-L. Lu, H.-J. Gao, S. K. Shaikhutdinov, H.-J. Freund, Phys. Rev. B 73 (2006) 165414.

${ }^{222}$ J. Schoiswohl, S. Surnev, M. Sock, S. Eck, M. G. Ramsey, F. P. Netzer, G. Kresse, Phys. Rev. B 71 (2005) 165437.

${ }^{223}$ S. Surnev, G. Kresse, M. Sock, M. G. Ramsey, F.P Netzer, Surf. Sci. 495 (2001) 91.

${ }^{224}$ A.-C. Dupuis, M. Abu Al-Haija, B. Richter, H. Kuhlenbeck, Freund, Surf. Sci. 539 (2003) 99.

${ }^{225}$ J. Schoiswohl, M. Sock, S. Surnev, M. G. Ramsey, F. P. Netzer, G. Kresse, J. N. Andersen, Surf. Sci. 555 (2004) 101.

${ }^{226}$ S. Surnev, L. Vitali, M. G. Ramsey, F. Netzer, G. Kresse, J. Hafner, Phys. Rev. B 61 (2000) 13945.

${ }^{227}$ F. P. Leisenberger, S. Surnev, L. Vitali, M. G. Ramsey, F. P. Netzer, J. Vac. Technol. A 17 (1999) 1743.

${ }^{228}$ C. Klein, G. Kresse, S. Surnev, F. P. Netzer, M. Schmid, P. Varga, Phys. Rev. B 68 (2003) 235416.

${ }^{229}$ S. Guimond, D. Göbke, Y. Romanyshyn, J. M. Sturm, M. Naschitzki, H. Kuhlenbeck, H.-J. Freund, J. Phys. Chem. C 112 (2008) 12363; S. Guimond, J. M. Sturm, D. Göbke, Y. Romanyshyn, M. Naschitzki, H. Kuhlenbeck, H.-J. Freund, J. Phys. Chem. C 112 (2008) 11835.

${ }^{230}$ M. Li, W. Hebenstreit, U. Diebold, A. M. Tyryshkin, M. K. Bowman, G. G. Dunham, M. A. Henderson, J. Phys. Chem. B 104 (2000) 4944.

${ }^{231}$ C. Africh, L. Köhler, F. Esch, M. Corso, C. Dri, T. Bucko, G. Kresse, G. Comelli, J. Am. Chem. Soc. 131 (2009) 3253.

${ }^{232}$ E. Vesselli, C. Africh, A. Baraldi, G. Comelli, F. Esch, R. Rosei, J. Chem. Phys. 114 (2001) 4221. 
${ }^{233}$ J. Gustafson, A. Mikkelsen, M. Borg, E. Lundgren, L. Kohler, G. Kresse, M. Schmid, P. Varga, J. Yuhara, X. Torrelles, C. Quiros, J. N. Andersen, Phys. Rev. Lett. 92 (2004) 126102.

${ }^{234}$ W. X. Li, W. Österlund, E. K. Vestergaard, R.T. Vang, J. Matthiesen, T.M. Pedersen, E. Laegsgaard, B. Hammer, F. Besenbacher, Phys. Rev. Lett. 93 (2004) 146104.

${ }^{235}$ B. L. M. Hendriksen, J. W. M. Frenken, Phys. Rev. Lett. 89 (2002) 046101.

${ }^{236}$ R.-P. Blum, H. Niehus, C. Hucho, R. Fortrie, M. V. Ganduglia-Pirovano, J. Sauer, S. Shaikhutdinov, H.-J. Freund, Phys. Rev. Lett. 99 (2007) 226103.

${ }^{237}$ A. R. Lennie, N. G. Condon, F. M. Leibsle, P. W. Murray, G. Thornton, D. J. Vaughan, Phys. Rev. B 53 (1996) 10244.

${ }^{238}$ N. G. Condon, F. M. Leibsle, T. Parker, A. R. Lennie, D. J. Vaughan, G. Thornton, Phys. Rev. B 55 (1997) 15885.

${ }^{239}$ G. Lu, A. Linsebigler, J. T. Yates, J. Phys. Chem. 98 (1994) 11733.

${ }^{240}$ M. V. Ganduglia-Pirovano, J. L. Da Silva, J. Sauer, Phys. Rev. Lett. 102 (2009) 026101.

${ }^{241}$ M. V. Ganduglia-Pirovano, J. Sauer, Phys. Rev. B 70 (2004) 045422.

${ }^{242}$ A. Pfau, K. D. Schierbaum, Surf. Sci. 321 (1994) 71.

${ }^{243}$ P. Sushko, J. L. Gavartin, A. L. Shluger, J. Phys. Chem. B 106 (2002) 2269.

${ }^{244}$ M. A. Henderson, J. M. White, H. Uetsuka, H. Onishi, J. Am. Chem. Soc. 125 (2003) 14975; J. M. White, M. A. Henderson, J. Phys. Chem. B 109 (2005) 14990.

${ }^{245}$ P. E. Trevisanutto, P. V. Sushko, A. L. Shluger, K. M. Beck, M. Henyk, A. G. Joly, W. P. Hess, Surf. Sci. 593 (2005) 210.

${ }^{246}$ P. Riccardi, M. Ishimoto, P. Barone, R A. Baragiola, Surf. Sci. 571 (2004) L305.

247 J. Kramer, C. Tegenkamp, H. Pfnür, Phys. Rev. B 67 (2003) 235401.

${ }^{248}$ J. Kramer, W. Ernst, C. Tegenkamp, H. Pfnür, Surf. Sci. 517 (2002) 87.

${ }^{249}$ M. Sterrer, E. Fischbach, T. Risse, H.-J. Freund, Phys. Rev. Lett. 94 (2005) 186101.

${ }^{250}$ C. Chusuei, X. Lai, K. Luo, D. W. Goodman, Top. Catal. 14 (2001) 71.

${ }^{251}$ G. Haas, A. Menck, H. Brune, J. V. Barth, J. A. Venables, K. Kern, Phys. Rev. B 61 (2000) 11105.

${ }^{252}$ H. M. Benia, X. Lin, H. J. Gao, N. Nilius, H.-J. Freund, J. Phys. Chem. C 111 (2007) 10528.

${ }^{253}$ M. Bäumer, M. Frank, M. Heemeier, R. Kühnemuth, S. Stempel, H. -J. Freund, Surf. Sci. 454 (2000) 957.

${ }^{254}$ S. Fischer, A. W. Munz, K. D. Schierbaum, W. Göpel, Surf. Sci. 337 (1995) 17.

${ }^{255}$ M. Kuhn, J. F. Anderson, J. Lehman, T. Mahmoud, U. Diebold, Epitaxial Oxide Thin Films III, 474 (1997) 359.

${ }^{256}$ R. Schaub, P. Thostrup, N. Lopez, E. Laegsgaard, I. Stensgaard, J.K. Norskov, F. Besenbacher, Phys. Rev. Lett. 87 (2001) 266104.

${ }^{257}$ O. Bikondoa, C. L. Pang, R. Ithnin, C. A. Muryn, H. Onishi, G. Thornton, Nat. Mater. 5 (2006) 189.

${ }^{258}$ G. Teobaldi, W. A. Hofer, O. Bikondoa, C. L. Pang, G. Cabailh, G. Thornton, Chem. Phys. Lett. 437 (2007) 73.

${ }^{259}$ M. Batzill, K. Katsiev, D. J. Gaspar, U. Diebold, Phys. Rev. B 66 (2002) 235401.

${ }^{260}$ P. Dudin, A. Barinov, L. Gregoratti, M. Kiskinova, F. Esch, C. Dri, C. Africh, G. Comelli, J. Phys. Chem. B 109 (2005) 13649.

${ }^{261}$ J.-L. Lu, H.-J. Gao, S. Shaikhutdinov, H.-J. Freund, Surf. Sci. 600 (2006) 5004.

${ }^{262}$ N. Nilius, V. Ganduglia-Pirovano, V. Bradzova, V. Simic-Milosevic, J. Sauer, H.-J. Freund, New J. Phys. (2009).

${ }^{263}$ M. Preisinger, J. Moosburger-Will, M. Klemm, S. Klimm, S. Horn, Phys. Rev. B 69 (2004) 075423.

${ }^{264}$ S. K. Shaikhutdinov, M. Ritter, X. G. Wang, H. Over, W. Weiss, Phys. Rev. B 60 (1999) 11062.

${ }^{265}$ N. Berdunov, S. Murphy, G. Mariotto, I. V. Shvets, Phys. Rev. Lett. 93 (2004) 057201.

${ }^{266}$ M. Sterrer, E. Fischbach, M. Heyde, N. Nilius, H.-P. Rust, T. Risse, H.-J. Freund, J. Phys. Chem. B 110 (2006) 8665.

${ }^{267}$ A. Stashans, E. Kotomin, J.-L. Calais, Phys. Rev. B 49 (1994) 14854.

${ }^{268}$ S. Schauermann, V. Johánek, M. Laurin, J. Libuda, H.-J. Freund, Phys. Chem. Chem. Phys. 5 (2003) 5139. 
${ }^{269}$ O. Y. Kolesnychenko, Y. A. Kolesnichenko, O. I. Shklyarevskii, H. van Kempen, Physica, B 291 (2000) 246.

270 J. M. Pitarke, F. Flores, P. M. Echenique, Surf. Sci. 234 (1990) 1.

${ }^{271}$ J. Goniakowski, F. Finocchi, C. Noguera, Rep. Progr. Phys. 71 (2008) 016501.

${ }^{272}$ P. W. Tasker, J. Phys.C 12 (1979) 4977.

${ }^{273}$ D. Wolf, Phys. Rev. Lett. 68 (1992) 3315.

${ }^{274}$ F. Finocchi, A. Barbier, J. Jupille, C. Noguera, Phys. Rev. Lett. 92 (2004) 136101.

${ }^{275}$ A. Barbier, G. Renaud, Surf. Sci. 392 (1997) L15; A. Barbier, G. Renaud, C. Mocuta, A. Stierle, Surf. Sci. 433 (1999) 761.

${ }^{276}$ F. Bottin, F. Finocchi, C. Noguera, Phys. Rev. B 68 (2003) 035418.

${ }^{277}$ X. G. Wang, A. Chaka, M. Scheffler, Phys. Rev. Lett. 84 (2000) 3650.

${ }^{278}$ J. Goniakowski, C. Noguera, L. Giordano, Phys. Rev. Lett. 98 (2007) 205701.

${ }^{279}$ B. Meyer, D. Marx, Phys. Rev. B 69 (2004) 235420.

${ }^{280}$ J. Goniakowski, C. Noguera, Phys. Rev. B 66 (2002) 085417.

${ }^{281}$ A. Pojani, F. Finocchi, J. Goniakowski, C. Noguera, Surf. Sci. 387 (1997) 354.

${ }^{282}$ H.-C. Poon, X. F. Hu, S. E. Chamberlin, D. K. Saldin, C. J. Hirschmugl, Surf. Sci. 600 (2006) 2505.

${ }^{283}$ R. Hacquart, J. Jupille, Chem. Phys. Lett. 439 (2007) 91.

${ }^{284}$ N. Kitakatsu, V. Maurice, P. Marcus, Surf. Sci. 411 (1998) 215.

${ }^{285}$ F. Rohr, K. Wirth, J. Libuda, D. Cappus, M. Bäumer, H.-J. Freund, Surf. Sci. 315 (1994) L977.

${ }^{286}$ Y. Wang, Z. Phys. Chem. 222 (2008) 927.

${ }^{287}$ M. Kiguchi, S. Entani, K. Saiki, T. Goto, A. Koma, Phys. Rev. B 68 (2003) 115402.

${ }^{288}$ R. Arita, Y. Tanida, S. Entani, M. Kiguchi, K. Saiki, H. Aoki, Phys. Rev. B 69 (2004) 235423.

${ }^{289}$ C. Tusche, H. L. Meyerheim, J. Kirschner, Phys. Rev. Lett. 99 (2007) 026102.

${ }^{290}$ T. M. Parker, N. G. Condon, R. Lindsay, F. M. Leibsle, G. Thornton, Surf. Sci. 415 (1998) L1046.

${ }^{291}$ O. Dulub, U. Diebold, G. Kresse, Phys. Rev. Lett. 90 (2003) 016102.

${ }^{292}$ G. Kresse, U. Diebold, Phys. Rev. B 68 (2003) 245409.

${ }^{293}$ G. Mariotto, S. Murphy, I. V. Shvets, Phys. Rev. B 66 (2002) 245426.

${ }^{294}$ W. Meyer, K. Biedermann, M. Gubo, L. Hammer, K. Heinz, J. Phys. Condens. Mat. 20 (2008) 265011.

${ }^{295}$ G. H. Vurens, M. Salmeron, G. A. Somorjai, Surf. Sci. 201 (1988) 129.

${ }^{296}$ G. Parteder, F. Allegretti, S. Surnev, F. P. Netzer, Surf. Sci. 602 (2008) 2666.

${ }^{297}$ R. S. Becker, J. A. Golovchenko, B. S. Swartzentruber, Phys. Rev. Lett. 55 (1985) 987.

${ }^{298}$ L. Olesen, M. Brandbyge, M. R. Sorensen, K. W. Jacobsen, E. Laegsgaard, I. Stensgaard, F. Besenbacher, Phys. Rev. Lett. 76 (1996) 1485.

${ }^{299}$ G. Kresse, J. Hafner, Phys. Rev. B 47 (1993) 558; G. Kresse, J. Furthmüller, Phys. Rev. B 54 (1996) 11169; P. E. Blöchl, Phys. Rev. B 50 (1994) 17953.

${ }^{300}$ S. L. Dudarev, G. A. Botton, S. Y. Savrasov, C. J. Humphreys, A. P. Sutton, Phys. Rev. B 57 (1998) 1505.

${ }^{301}$ E. D. L. Rienks, N. Nilius, L. Giordano, J. Goniakowski, G. Pacchioni, M. P. Felicissimo, T. Risse, H.-P. Rust, H.-J. Freund, Phys. Rev. B 75 (2007) 205443.

${ }^{302}$ W. Zhang, Z. Li, Y. Luo. J. Yang, J. Phys. Chem .(2009) in press.

${ }^{303}$ L. R. Merte, J. Knudsen, L. C. Grabow, R. T. Vang, E. Laegsgaard, M. Mavrikakis, F. Besenbacher, Surf. Sci. 603 (2009) L15.

${ }^{304}$ Y. Xu, J.Li, Y. Zhang, W. Chen, Surf. Sci. 525 (2003) 13.

${ }^{305}$ K. M. Neyman, S. P. Ruzankin, N. Rösch, Chem. Phys. Lett. 246 (1995) 546; K. M. Neyman, N. Rösch, Chem. Phys. 168 (1992) 267.

${ }^{306}$ C. Di Valentin, G. Pacchioni, T. Bredow, D. Dominguez-Ariza, F. Illas, J. Chem. Phys. 117 (2002) 2299.

${ }^{307}$ F. Winkelmann, S. Wohlrab, J. Libuda, M. Bäumer, D. Cappus, M. Menges, K. Al-Shamery, H. Kuhlenbeck, H.-J. Freund, Surf. Sci. 307 (1994) 1148.

${ }^{308}$ R. Wichtendahl, M. Rodriguez-Rodrigo, U. Härtel, H. Kuhlenbeck, H.-J. Freund, Surf. Sci. 423 (1999) 90. 
${ }^{309}$ R. Wichtendahl, M. Rodriguez-Rodrigo, U. Härtel, H. Kuhlenbeck, H.-J. Freund, Phys. Sat. Solid. 173 (1999) 93.

${ }^{310}$ C. T. Campbell, D. E. Starr, J. Am. Chem. Soc. 124 (2002) 9212; J. H. Larsen, J. T. Ranney, D. E. Starr, J. E. Musgrove, C. T. Campbell, Phys. Rev. B 63 (2001) 195410.

${ }^{311}$ B. Meyer, D. Marx, O. Dulub, U. Diebold, M. Kunat, D. Langenberg, C. Wöll, Angew. Chem. Int. Ed. 43 (2004) 6641.

${ }^{312}$ I. Yudanov, G. Pacchioni, K. Neyman, N. Rösch, J. Phys. Chem. B 101 (1997) 2786.

${ }^{313}$ L. Giordano, G. Pacchioni, T. Bredow, J. F. Sanz, Surf. Sci. 471 (2000) 21.

${ }^{314}$ N. C. Hernandez, J. Graciani, A. Marquez, J. F. Sanz, Surf. Sci. 575 (2005) 189.

${ }^{315}$ C. Inntam, L. V. Moskaleva, K. M. Neyman, V. A. Nasluzov, N. Rösch, Appl. Phys. A 82 (2006) 181.

${ }^{316}$ A. Del Vitto, G. Pacchioni, F. O. Delbecq, P. Sautet, J. Phys. Chem. B 109 (2005) 8040.

${ }^{317}$ V. A. Nasluzov, V. V. Rivanenkov, A. B. Gordienko, K. M. Neyman, U. Birkenheuer, N. Rösch, J. Chem. Phys. 115 (2001) 8157.

${ }^{318}$ K. M. Neyman, C. Inntam, A. V. Matveev, V. A. Nasluzov, N. Rösch, J. Am. Chem. Soc. 127 (2005) 11652.

${ }^{319}$ C. A. Scamehorn, A. C. Hess, M. I. McCarthy, J. Chem. Phys. 99 (1993) 2786; M. Sgroi, C. Pisani, M. Busso, Thin Sol. Films 400 (2001) 64.

${ }^{320}$ B. Meyer, H. Rabaa, D. Marx, Phys. Chem. Chem. Phys. 8 (2006) 1513.

${ }^{321}$ G. Pacchioni, G. Cogliandro, P.S. Bagus, Surf. Sci. 255 (1991) 344.

${ }^{322}$ D. Matthey, J. W. Wang, S. Wendt, J. Matthiesen, R. Schaub, E. Laegsgaard, B. Hammer, F. Besenbacher, Science 315 (2007) 1692.

${ }^{323}$ M. Sterrer, T. Risse, H.-J. Freund, Appl. Catal. A 307 (2006) 58.

${ }^{324}$ A. V. Matveev, K. M. Neyman, I. V. Yudanov, N. Rösch, Surf. Sci. 426 (1999) 123.

${ }^{325}$ N. Lopez, F. Illas, G. Pacchioni, J. Am. Chem. Soc. 121 (1999) 813.

${ }^{326}$ S. Abbet, E. Riedo, H. Brune, U. Heiz, A. M. Ferrari, L. Giordano, G. Pacchioni, J. Am. Chem. Soc. 123 (2001) 6172.

${ }^{327}$ L. Giordano, J. Goniakowski, G. Pacchioni, Phys. Rev. B 64 (2001) 075417; L. Giordano, C. Di Valentin, J. Goniakowski, G. Pacchioni, Phys. Rev. Lett. 92 (2004) 096105.

${ }^{328}$ U. Martinez, L. Giordano, G. Pacchioni, J. Phys. Chem. B 110 (2006) 17015.

${ }^{329}$ C. Zhang, A. Michaelides, D. A. King, S. J. Jenkins, J. Chem. Phys. 129 (2008) 194708.

${ }^{330}$ M. A. Henderson, Langmuir 12 (1996) 5093; W. Langel, Surf. Sci. 496 (2002) 141.

${ }^{331}$ P. J. D. Lindan, N. M. Harrison, J. M. Holender, M. J. Gillan, Chem. Phys. Lett. 261 (1996) 246.

${ }^{332}$ J. T. Stuckless, D. E. Starr, D. J. Bald, C. T. Campbell, J. Chem. Phys. 107 (1997) 5547.

${ }^{333}$ M. Valden, X. Lai, D. W. Goodman, Science 281 (1998) 1647.

${ }^{334}$ M. Haruta, T. Kobayashi, H. Sano, N. Yamada, Chem. Lett. 405 (1987); M. Haruta, Cat. Tech. 6, $102(2002)$.

${ }^{335}$ X. Lai, T. P. St Clair, M. Valden, D. W. Goodman, Prog. Surf. Sci. 59 (1998) 25.

${ }^{336}$ A. K. Santra, F. Yang, D.W. Goodman, Surf. Sci. 548 (2004) 324.

${ }^{337}$ C. Xu, X. Lai, G. W. Zajac, D. W. Goodman, Phys. Rev. B 56 (1997) 13464.

${ }^{338}$ K. Luo, T. P. St Clair, X. Lai, D. W. Goodman, J. Phys. Chem. B 104 (2000) 3050.

${ }^{339}$ A. Howard, C. E. J. Mitchell, R. G. Egdell, Surf. Sci. 515 (2002) L504.

${ }^{340}$ X. F. Lai, T. P. St Clair, D. W. Goodman, Farad. Discuss. 114 (1999) 279; A. Berko, J. Szoko, F. Solymosi, Surf. Sci. 566 (2004) 337.

${ }^{341}$ R. A. Bennett, P. Stone, M. Bowker, Catal. Lett. 59 (1999) 99.

${ }^{342}$ O. Dulub, W. Hebenstreit, U. Diebold, Phys. Rev. Lett. 84 (2000) 3646.

${ }^{343}$ E. Wahlström, N. Lopez, R. Schaub, P. Thostrup, A. Ronnau, C. Africh, E. Laegsgaard, J.K. Norskov, F. Besenbacher, Phys. Rev. Lett. 90 (2003) 026101.

${ }^{344}$ D. Matthey, J. W. Wang, S. Wendt, J. Matthiesen, R. Schaub, E. Laegsgaard, B. Hammer, F. Besenbacher, Science 315 (2007) 1692.

${ }^{345}$ L. Benz, X. Tong, P. Kemper, H. Metiu, M. T. Bowers, S. K. Buratto, J. Phys. Chem. B 110 (2006) 663. 
${ }^{346}$ X. Tong, L. Benz, S. Chretien, P. Kemper, A. Kolmakov, H. Metiu, M. T. Bowers, S. K. Buratto, J. Chem. Phys. 123 (2005) 204701.

${ }^{347}$ D. E. Ollis, H. Al-Ekabi [Eds.], Photo-catalytic treatment of water and air, (Elsevier, Amsterdam, 1993).

${ }^{348}$ I. M. Brookes, C. A. Muryn, G. Thornton, Phys. Rev. Lett. 87 (2001) 26.

${ }^{349}$ S. Suzuki, K. Fukui, H. Onishi, Y. Iwasawa, Phys. Rev. Lett. 84 (2000) 2156.

${ }^{350}$ S. Wendt, J. Matthiesen, R. Schaub, E. K. Vestergaard, E. Lægsgaard, F. Besenbacher, B. Hammer, Phys. Rev. Lett. 96 (2006) 066107.

${ }^{351}$ Z. Zhang, O. Bondarchuk, B. D. Kay, J. M. White, Z. Dohnalek, J. Phys. Chem. B 110 (2006) 21840.

${ }^{352}$ P. Maksymovych, S. Mezhenny, J. T. Yates, Chem. Phys. Lett. 382 (2003) 270.

${ }^{353}$ E. Wahlström, E. K. Vestergaard, R. Schaub, A. Ronnau, M. Vestergaard, E. Laegsgaard, I. Stensgaard, F. Besenbacher, Science 303 (2004) 511.

${ }^{354}$ E. D. L. Hebenstreit, W. Hebenstreit, U. Diebold, Surf. Sci. 470 (2001) 347.

${ }^{355}$ P. W. Murray, N. G. Condon, G. Thornton, Surf. Sci. 323 (1995) L281.

${ }^{356}$ A. Tekiel, J. S. Prauzner-Bechcicki, S. Godlewski, J. Budzioch, M. Szymonski, J. Phys. Chem. C 112 (2008) 12606.

${ }^{357}$ Z. R. Zhang, O. Bondarchuk, J. M. White, B. D. Kay, Z. Dohnalek, J. Am. Chem. Soc. 128 (2006) 4198.

${ }^{358}$ Z. Zhang, O. Bondarchuk, B. D. Kay, J. M. White, Z. Dohnalek, J. Phys. Chem. C 111 (2007) 3021.

${ }^{359}$ M. A. Henderson, J. M. White, H. Uetsuka, H. Onishi, J. Am. Chem. Soc. 125 (2003) 14974.

${ }^{360}$ M. Lackinger, M. Janson, W. Ho, to be published.

${ }^{361}$ O. Dulub, M. Batzill, U. Diebold, Top. Catal. 36 (2005) 65; L. V. Koplitz, O. Dulub, U. Diebold, J. Phys. Chem. B 107 (2003) 10583.

${ }^{362}$ M. Kroll, U. Köhler, Surf. Sci. 601 (2007) 2182.

${ }^{363}$ X. L. Yin, A. Birkner, K. Hanel, T. Lober, U. Köhler, C. Wöll, Phys. Chem. Chem. Phys. 8 (2006) 1477.

${ }^{364}$ O. Dulub, B. Meyer, U. Diebold, Phys. Rev. Lett. 95 (2005) 136101.

${ }^{365}$ R. S. Cutting, C. A. Muryn, D. J. Vaughan, G. Thornton, Surf. Sci. 602 (2008) 1155.

${ }^{366}$ R. E. Tanner, Y. Liang, E. I. Altman, Surf. Sci. 506 (2002) 251.

${ }^{367}$ H.-J. Freund, G. Pacchioni, Chem. Soc. Rev. 37 (2008) 2224.

${ }^{368}$ P. Frondelius, H. Häkkinen, K. Honkala, New J. Physics 9 (2007) 339.

${ }^{369}$ A. S. Wörz, U. Heiz, F. Cinquini, G. Pacchioni, J. Phys. Chem. B. 109 (2005) 18418.

${ }^{370}$ L. Giordano, U. Martinez, S. Sicolo, G. Pacchioni, J. Chem. Phys. 127 (2007) 144713.

${ }^{371}$ L. Giordano, G. Pacchioni, Phys. Chem. Chem. Phys. 8 (2006) 3335.

${ }^{372}$ L. Giordano, G. Pacchioni, J. Goniakowski, N. Nilius, E. D. L. Rienks, H.-J. Freund, Phys. Rev. Lett. 101 (2008) 026102.

${ }^{373}$ N. Cabrera, N. F. Mott, Rep. Prog. Phys. 12 (1948) 163.

${ }^{374}$ M. Sterrer, T. Risse, U. M. Pozzoni, L. Giordano, M. Heyde, H.-P. Rust, G. Pacchioni, H.-J. Freund, Phys. Rev. Lett. 98 (2007) 096107.

${ }^{375}$ N. Nilius, V. G. Pirovano, V. Bradzova, M. Kulawik, J. Sauer, H.-J. Freund, Phys. Rev. Lett. 100 (2008) 096802.

${ }^{376}$ M. Walter, P. Frondelius, K. Honkala, H. Hakkinen, Phys. Rev. Lett. 99 (2007) 096102.

${ }^{377}$ D. Ricci, A. Bongiorno, G. Pacchioni, U. Landman, Phys. Rev. Lett. 97 (2006) 036106.

${ }^{378}$ U. Martinez, L. Giordano, G. Pacchioni, J. Chem. Phys. 128 (2008) 164707.

${ }^{379}$ U. Martinez, J. F. Jerratsch, N. Nilius, L. Giordano, G. Pacchioni, H.-J. Freund, Phys. Rev. Lett. (2009) in press.

380 J. R. Gomes, F. Illas, N. C. Hernandez, A. Marquez, J. F. Sanz, Phys. Rev. B 65 (2002) 125414.

${ }^{381}$ L. Giordano, A. Del Vitto, G. Pacchioni, J. Chem. Phys. 124 (2006) 034701.

${ }^{382}$ H. P. Bonzel, Surf. Sci. Rep. 8 (1987) 43; D. Diehl, R. McGrath, Surf. Sci. Rep. 23 (1996) 43.

383 J. Kliewer, R. Berndt, Surf. Sci. 477 (2001) 250.

${ }^{384}$ R. J. Hamers, J. Vac. Sci. Technol. B 6 (1988) 1462. 
${ }^{385}$ M. Sterrer, T. Risse, L. Giordano, M. Heyde, N. Nilius, H.-P. Rust, G. Pacchioni, H.-J. Freund, Angew. Chem. Int. Ed. 46 (2007) 8703.

${ }^{386}$ N. Nilius, V. G. Pirovano, V. Bradzova, M. Kulawik, J. Sauer, H.-J. Freund, submitted.

${ }^{387}$ M. Kulawik, N. Nilius, H.-J. Freund, Phys. Rev. Lett. 96 (2006) 036103.

${ }^{388}$ V. Simic-Milosevic, M. Heyde, N. Nilius, M. Sterrer, T. Risse, H.-J. Freund, L. Giordano, G. Pacchioni, J. Am. Chem. Soc. 130 (2008) 7814.

${ }^{389}$ V. Simic-Milosevic, M. Heyde, X. Lin, T. König, H.-P. Rust, M. Sterrer, T. Risse, N. Nilius, H.-J. Freund, L. Giordano, G. Pacchioni, Phys. Rev. B 78 (2008) 235429.

${ }^{390}$ M. Sterrer, T. Risse, M. Yulikov, H.-J. Freund, J. Carrasco, F. Illas, C. Di Valentin, L. Giordano, G. Pacchioni, Angew. Chem. Int. Ed. 45 (2006) 2695.

${ }^{391}$ N. Nilius, T. M. Wallis, W. Ho, Phys. Rev. Lett. 90 (2003) 046808.

${ }^{392}$ T. M. Wallis, N. Nilius, and W. Ho, Phys. Rev. Lett. 89 (2002) 236802.

${ }^{393}$ M. Sterrer, T. Risse, M. Heyde, H.-P. Rust, H.-J. Freund, Phys. Rev. Lett. 98 (2007) 206103.

${ }^{394}$ M. Yulikov, M. Sterrer, M. Heyde, H.-P. Rust, T. Risse, H.-J. Freund, G. Pacchioni, A. Scagnelli, Phys. Rev. Lett. 96 (2006) 146804.

${ }^{395}$ X. Lin, N. Nilius, H.-J. Freund, M. Walter, P. Frondelius, K. Honkala, H. Häkkinen, Phys. Rev. Lett. 102 (2009) 206801.

${ }^{396}$ W. A. de Heer, Rev. Mod. Phys. 65 (1993) 611.

${ }^{397}$ K. Honkala, H. Häkkinen, J. Phys. Chem. 111 (2007) 4319.

${ }^{398}$ S. W. Wu, N. Ogawa, G. V. Nazin, W. Ho, J. Phys. Chem. C 112 (2008) 5241.

${ }^{399}$ G. Mikaelian, N. Ogawa, X. W. Tu, W. Ho, J. Chem. Phys. 124 (2006) 131101.

${ }^{400}$ S.W. Wu, N. Ogawa, W. Ho, Science 312 (2006) 1362.

${ }^{401}$ G. Cuniberti, G. Fagas, K. Richter [Eds.], Introducing molecular electronics, (Springer, Berlin, 2005).

${ }^{402}$ V. Maurice, S. Cadot, P. Marcus, Surf. Sci. 471 (2001) 43.

${ }^{403}$ N. Nilius, E. Rienks, H.-P. Rust, H.-J. Freund, Phys. Rev. Lett. 95 (2005) 066101.

${ }^{404}$ X. Lin, N. Nilius, J. Phys. Chem. C 112 (2008) 15325.

${ }^{405}$ F. Silly, M. Pivetta, M. Ternes, F. Patthey, J. P. Pelz, and W. D. Schneider, Phys. Rev. Lett. 92 (2004) 016101.

${ }^{406}$ M. Kulawik, M. Heyde, N. Nilius, H.-P. Rust, H.-J. Freund, B. Mantooth, P. S. Weiss, Surf. Sci. 590 (2005) L253.

${ }^{407}$ M. Persson, Phys. Rev. B 72, (2005) R081404.

${ }^{408}$ G. Witte, C. Wöll, Mater. Res. 19 (2004) 1889.

${ }^{409}$ C. Y. Ruan, V. Mastryukov, M. J. Fink, J. Chem. Phys. 111 (1999) 3035; J. Janczak, R. Kubiak, Polyhedron 20 (2001) 2901.

${ }^{410}$ J. V. Barth, G. Costantini, K. Kern, Nature 437 (2005) 671; A. Dmitriev, H. Spillmann, N. Lin, J. V. Barth, K. Kern, Angew. Chem. Int. Ed. 42 (2003) 2670.

${ }^{411}$ N. Berdunov, G. Mariotto, K. Balakrishnan, S. Murphy, I. V. Shvets, Surf. Sci. 600 (2006) L287.

${ }^{412}$ C. Becker, A. Rosenhahn, A. Wiltner, K. Bergmann, J. Schneider, P. Pervan, M. Milun, M. Kralj, K. Wandelt, New J. Phys. 4 (2002) 75.1

${ }^{413}$ S. Degen, C. Becker, K. Wandelt, Faraday Discuss. 125 (2004) 343; G. Hamm, C. Becker, C. R. Henry, Nanotechnol 17 (2006) 1943.

${ }^{414}$ A. Rosenhahn, J. Schneider, C. Becker, K. Wandelt, Appl. Surf. Sci. 142 (1999) 169.

${ }^{415}$ T. Maroutian, S. Degen, C. Becker, K. Wandelt, R. Berndt, Phys. Rev. B 68 (2003) 155414.

${ }^{416}$ F. Sedona, M. Sambi, L. Artiglia, G. A. Rizzi, A Vittadini, A. Fortunelli, G. Granozzi, J. Phys. Chem. C 112 (2008) 3187.

${ }^{417}$ M. Baron, D. Stacchiola, S. Ulrich, N. Nilius, S. Shaikhutdinov, H.-J. Freund, U. Martinez, L.Giordano, G. Pacchioni, Chem. Phys. C 112 (2008) 3405.

${ }^{418}$ S. Ulrich, N. Nilius, H.-J. Freund, L. Giordano, G. Pacchioni, ChemPhysChem. 9 (2008) 1367.

${ }^{419}$ F. Yang, E. Trufan, R. D. Adams, D. W. Goodman, J. Phys. Chem. C 112 (2008) 14233.

${ }^{420}$ D. W. Breck, Zeolite Molecular Sieves: Structure, Chemistry, and Use, (New York, 1974, Wiley).

${ }^{421}$ T. Schröder, M. Adelt, B. Richter, M. Naschitzki, M. Bäumer, H.-J. Freund, Surf. Rev. \& Lett.7 ( 2000) 7. 
${ }^{422}$ J. Weissenrieder, S. Kaya, J.L. Lu, H. Gao, S. Shaikhutdinov, H.-J. Freund, M. Sierka, T. K. Todorova, J. Sauer, J. Phys. Rev. Lett. 95 (2005) 076103.

${ }^{423}$ S. Ulrich, N. Nilius, H.-J. Freund, U. Martinez, L. Giordano, G. Pacchioni, Surf. Sci. 603 (2009) 1145.

${ }^{424}$ K. Min, W. T. Wallace, A. K. Santra, D. W. Goodman, J. Phys. Chem. B 108 (2004) 16339.

${ }^{425}$ D. R. Lide [Ed.], Handbook of chemistry and physics, CRC Press, Boca Raton, 2008.

${ }^{426}$ S. Ulrich, N. Nilius, H.-J. Freund, U. Martinez, L. Giordano, G. Pacchioni, Phys. Rev. Lett. 102 (2009) 016102

${ }^{427}$ J. X. Wang, J. H. Lunsford, J. Phys. Chem. 90 (1986) 5883.

${ }^{428}$ M. C. Wu, C. M. Truong, D. W. Goodman, Phys. Rev. B 46 (1992) 12688; B. K. Min, W. T. Wallace, D. W. Goodman, J. Chem. Phys. B 108 (2004) 14609.

${ }^{429}$ T. Schalow, B. Brandt, D. E. Starr, M. Laurin, S. K. Shaikhutdinov, S. Schauermann, J. Libuda, H.-J. Freund, Phys. Chem. Chem. Phys. 9 (2007) 1347.

${ }^{430}$ M. L. Knotek, P. J. Feibelman, Phys. Rev. Lett. 40 (1978) 964.

${ }^{431}$ J. L. Lu, H. J. Gao, S. Shaikhutdinov, H.-J. Freund, Catal. Lett. 114 (2007) 8.

${ }^{432}$ Y. Romanyshyn, S. Guimond, H. Kuhlenbeck, S. Kaya, R.P. Blum, H. Niehus, S. Shaikhutdinov, V. Simic Milosevic, N. Nilius, H.-J. Freund, M. V. Ganduglia-Pirovano, R. Fortrie, J. Döbler, J. Sauer, Top. Catal. 50 (2008) 106.

${ }^{433}$ N. Nilius, V. Simic-Milosevic, J. Phys. Chem. C 112 (2008) 10027.

${ }^{434}$ M. Abu Haija, S. Guimond, A. Uhl, H. Kuhlenbeck, H.-J. Freund, J. K. Todorova, M. V. Ganduglia-Pirovano, J. Döbler, J. Sauer, Surf. Sci. 600 (2006) 1497.

435 J. Ashkenazi, M. Weger, Adv Phys. 22 (1973) 207; S. Y. Ezhov, V. I. Anisimov, D. I. Khomskii, G. A. Sawatzky, Phys. Rev. Lett. 83 (1999) 4136.

${ }^{436}$ G.A. Thomas, D.H. Rapkine, S.A. Carter, A.J. Millis, T.F. Rosenbaum, P. Metcalf, J.M. Honig, Phys. Rev. Lett. 73 (1994) 1529.

${ }^{437}$ R. Shi, C. Asahi, C. Stampfl, Phys. Rev. B 75 (2007) 205125.

${ }^{438}$ J. Repp, G. Meyer, Phys. Rev. Lett. 94 (2005) 026803; P. Liljerith, J. Repp, G. Meyer, Science 317 (2007) 1203.

${ }^{439}$ M.-S. Liao, S. Schreiner, J. Chem. Phys. 114 (2001) 9780.

${ }^{440}$ J. M. Sturm, D. Göbke, H. Kuhlenbeck, J. Döbler, U. Reinhardt, M. V. Ganduglia-Pirovano, J. Sauer, H.-J. Freund, Phys. Chem. Chem. Phys. 11 (2009) 3290.

${ }^{441}$ D. Göbke, Y. Romanyshyn, S. Guimond, J. M. Sturm, H. Kuhlenbeck, J. Döbler, U. Reinhardt, M. V. Ganduglia-Pirovano, J. Sauer, H.-J. Freund, Angew. Chem. Int. Ed. 48 (2009) 3695.

${ }^{442}$ A. Bandara, M. Abu-Haija, F. Höbel, H. Kuhlenbeck, R. Rupprechter, H.-J. Freund, Top. Catal. 46 (2007) 223.

${ }^{443}$ T. Bertrams, F. Winkelmann, T. Uttich, H.-J. Freund, H. Neddermeyer, Surf. Sci. 331 (1995) 1515.

${ }^{444}$ R. L Schwöbel, E. J. Shipsey, J. Appl. Phys. 37 (1996) 3682; G. Ehrlich, K. Stolt, Ann. Rev. Phys. Chem. 31 (1980) 603.

${ }^{445}$ M. Heemeier, S. Stempel, S. Shaikhutdinov, J. Libuda, M. Bäumer, R. J. Oldman, S. D. Jackson, H.-J. Freund, Surf. Sci. 523 (2003) 103.

${ }^{446}$ V. Rose, V. Podgursky, R. David, R. Franchy, Surf. Sci. 601 (2007) 786.

${ }^{447}$ Z.-H. Qin, M. Lewandowski, Y.-N. Sun, S. Shaikhutdinov, H.-J. Freund, J. Phys. Chem. C 112 (2008) 10209.

${ }^{448}$ L. Vitali, M. A. Schneider, K. Kern, L. Wirtz, A. Rubio, Phys. Rev. B 69 (2004) 121414.

${ }^{449}$ A. Sternig, S. Stankic, M. Müller, J. Bernardi., E. Knözinger, O. Diwald, Adv. Mater. 20 (2008) 4840. 